UNIVERSIDADE DE SÃO PAULO

ESCOLA DE ARTES, CIÊNCIAS E HUMANIDADES

PROGRAMA DE PÓS-GRADUAÇÃO EM CIÊNCIAS DA ATIVIDADE FÍSICA

FERNANDO CARVALHEIRO REISER

Aplicação de abordagens de controle motor na análise temporal e de desempenho em arqueiros de elite 


\section{Aplicação de abordagens de controle motor na análise temporal e de desempenho em arqueiros de elite}

Dissertação apresentada a Escola de Artes, Ciências e Humanidades da Universidade de São Paulo para obtenção do título de Mestre em Ciências pelo Programa de PósGraduação em Ciências da Atividade Física.

Versão corrigida contendo as alterações solicitadas pela comissão julgadora em 11 de março de 2019. A versão original encontra-se em acervo reservado na Biblioteca da EACH/USP e na Biblioteca Digital de Teses e Dissertações da USP (BDTD), de acordo com a resolução CoPGr 6018, de 13 de outubro de 2011.

Área de concentração: Desempenho Motor

Orientador: Prof. Dr. Luis Mochizuki

SÃO PAULO 
Autorizo a reprodução e divulgação total ou parcial deste trabalho, por qualquer meio convencional ou eletrônico, para fins de estudo e pesquisa, desde que citada a fonte.

Reiser, Fernando Carvalheiro

Aplicação de abordagens de controle motor na análise temporal e de desempenho em arqueiros de elite / Fernando Carvalheiro Reiser ; orientador, Luis Mochizuki. - 2019. 133 f.: il.

Dissertação (Mestrado em Ciências) - Programa de PósGraduação em Ciências da Atividade Física, Escola de Artes, Ciências e Humanidades, Universidade de São Paulo, São Paulo Versão corrigida

1. Biomecânica. 2. Controle motor. 3. Desempenho motor. 4. Músculos. 5. Eletromiografia. 6. Tiro com arco.

I. Mochizuki, Luis, orient. II. Título 
Nome: REISER, Fernando Carvalheiro

Título: Aplicação de abordagens de controle motor na análise temporal e de desempenho em arqueiros de elite

Dissertação apresentada a Escola de Artes, Ciências e Humanidades da Universidade de São Paulo para obtenção do título de Mestre em Ciências pelo Programa de PósGraduação em Ciências da Atividade Física.

Área de concentração: Desempenho Motor

Comissão de examinadores:

Prof. Dr. Instituição:

Julgamento: Assinatura:

Prof. Dr. Instituição:

Julgamento: Assinatura:

Prof. Dr. Instituição:

Julgamento: Assinatura: 
Dedico este trabalho as mãos calejadas de meus pais... 


\section{AGRADECIMENTOS}

Agradeço primeiramente a Deus - Sancta Trinitas unus Deus - por tudo que fez Venite adoremos, Dominus! - e a Santa Virgem Maria que, por meio de seu saltério, ajudou-me e iluminou meu caminho, de um início de caminhada ateu para uma conversão que sequer esperava. Isto girou em $180^{\circ}$ minha compreensão tanto do meu mestrado como da minha vida.

Agradeço a minha família, meus pais Márcio Antônio Reiser e Graziele Carvalheiro Reiser pelo seu incondicional amor e apoio nesse empreendimento, a meus irmãos Gustavo Carvalheiro Reiser e Bruno Carvalheiro Reiser, pelas nossas conversas filosóficas e teológicas, a minha estimada avó Djanira Carvalheiro, por todo seu carinho, e meu avô José (in memorian) e minha tia-avó Terezinha (in memorian).

Agradeço a minha namorada, Beatriz e toda sua família (Sandra, Júlio, Gabriele e Lucas) que me acolheram com imenso carinho.

Agradeço ao meu excelente orientador e mestre Prof. Dr. Luis Mochizuki, por toda sua compreensão e trabalhoso empreendimento de orientar tal acadêmico atrapalhado e pedante como eu.

Agradeço ao Prof. Dr. Júlio Serrão, pelo incentivo e pelas conversas sempre produtivas que tivemos, ao Prof. Dr. Fernando Henrique Magalhães pela sua inestimável ajuda em todo projeto, igualmente ao Prof. Dr. Saldanha, ao competente Prof. Dr. Ulysses Ervilha pelos seus ensinamentos, ao grande mestre da Biomecânica Prof. Dr. Alberto Carlos Amadio, por ser referência nessa área, pelo seu imenso conhecimento e pelas nossas conversas. Ao Prof. Dr. Luciano Basso, pela troca de ideias estatísticas.

Aos amigos e companheiros de jornada acadêmica da EACH e EEFE, meu agradecimento em especial aos colegas Bruno Durante, João Manoel, Nadjila Tejo, Hamilton Miotto, Adilson, André, Riccardo, Demostenys da Silva, Jhonata Cesar, Bruno Mezêncio, Pedro Sampaio, Jumes Leopoldino, Carlos, Giordano e William Cordeiro de Souza.

Agradeço também pelo profissionalismo dos arqueiros da Seleção Brasileira que participaram do trabalho, dos técnicos Renzo e Evandro, e todos aqueles que ajudaram na coleta, e a CAPES pelo financiamento de parte desta pesquisa.

Agradeço aos confessores Pe. Leandro, Pe. Conrado, Pe. Ladi, Pe. Sebastião, Pe. Giovanni, e aos mestres Pe. Paulo Ricardo, Prof. Olavo de Carvalho, Prof. Rafael Falcon, Prof. Raphael de Paola e Prof. Luiz Gonzaga de Carvalho Neto pelos seus inestimáveis ensinamentos. 
Bernstein gostava de contar a seguinte história (conforme dito pelo Prof. Zatsiorsky): "Você provavelmente não sabe que Deus tem um primo que nunca foi muito famoso. Então, o primo pediu a Deus para ajudá-lo a alcançar fama e glória na ciência. Para agradar o primo, Deus lhe deu a capacidade de obter qualquer informação sobre sistemas físicos em pouco tempo e viajar para qualquer lugar dentro de um microssegundo. Primeiro, o primo decidiu verificar se havia vida em outros planetas. Sem problemas; Ele viajou para todos os planetas simultaneamente e obteve uma resposta. Então ele decidiu descobrir qual era o fundamento da matéria. Mais uma vez, isso foi fácil: ele se tornou extremamente pequeno, rastejou para dentro das partículas elementares, olhou em volta e uma resposta. Então, ele decidiu aprender como o cérebro humano controla os movimentos. Ele adquiriu a informação sobre todos os neurônios e suas conexões, sentou-se em sua mesa e olhou para a planta. Se a história está certa, ele ainda está sentado lá e olhando para o mapa das conexões neuronais". 


\section{RESUMO}

REISER, Fernando Carvalheiro. Aplicação de abordagens de controle motor na análise temporal e de desempenho em arqueiros de elite. 2019. 133 p. Dissertação (Mestrado em Ciências) - Escola de Artes, Ciências e Humanidades, Universidade de São Paulo - São Paulo/SP. 2019. Versão corrigida

O Tiro com Arco é uma modalidade olímpica na qual os atletas exibem diversas estratégias de coordenação muscular na preparação do disparo da flecha, principalmente no período da liberação do clicker, tornando dificultosa a interpretação da coordenação de arqueiros no que tange à sua pontuação. Deste modo, algumas técnicas, como a Análise de Vetor Codificado e co-ativação/inibição recíproca, provenientes de abordagens de Controle Motor, podem ajudar-nos a desvendar quais parâmetros em comum que estes arqueiros utilizam quando atiram e sua relação com seu desempenho obtido durante o disparo sequencial de flechas. O objetivo do trabalho foi o de avaliar a coordenação muscular utilizada por arqueiros da seleção brasileira durante uma simulação qualificatória olímpica, através da técnica eletromiográfica. Participaram 11 arqueiros da seleção brasileira; idade: 25,2 $\pm 4,9$ anos, altura: 1,72 $\pm 0,12 \mathrm{~m}$, peso: $68,6 \pm 11,8 \mathrm{~kg}$, FITA score: $1258 \pm 43$ pontos, Qualificação: 632 \pm 20 pontos, Flecha média: $8,7 \pm 0,3$ pontos. A análise EMG foi dividida em $0,5 \mathrm{~s}$ antes e $0,15 \mathrm{~s}$ após a liberação do clicker. Foi utilizado o valor RMS deste sinal de doze músculos: no lado que traciona a corda (dominante) - FSD (Flexor Superficial dos dedos); ED (Extensor dos dedos); BBL (Bíceps Braquial); TBL (Tríceps Braquial - Cabeça longa); GD (Grande Dorsal) PMC (Peitoral Maior - Clavicular); TS (Trapézio Superior); SAD (Serrátil Anterior); RA (Reto Abdominal) e ML (Multífido Lombar). Lado não dominante (nD) DPnD (Deltóide Posterior); SAnD (Serrátil Anterior), no qual foram criados em 14 pares de análise (agonista-antagonista: FSD-ED; BBL-TBL; GD-PMC; TS-SAD; RA-ML; distal-proximal: FSD-BBL; FSD-TBL; ED-BBL; ED-TBL; e mistos: PMC-SAD; GD-TS; SAD-SAnD; SAnD-DPnD; GD-DPnD), para a análise dentro das técnicas de Co-ativação/Inibição Reciproca e Análise de Vetor Codificado. Nos resultados; oito pares (FSD-ED; BBL-TBL; TS-SAD; FSD-BBL; FSD-TBL; EDTBL; SAD-SAnD; SAnD-DPnD) co-ativaram diferentemente na transição de blocos (p<0.05); e 12 (FSD-ED; BBL-TBL; GD-PMC; TS-SAD; FSD-BBL; FSD-TBL; EDBBL; ED-TBL; PMC-SAD; SAD-SAnD; SAnD-DPnD; GD-DPnD) co-ativaram diferentemente na comparação do escore obtido ( $p<0.05)$. Em nove pares, os arqueiros, evidenciaram diferentes estratégias de inibição recíproca na transição de blocos (FSD- 
ED; BBL-TBL; TS-SAD; FSD-BBL; FSD-TBL; ED-BBL; ED-TBL; SAD-SAnD; SAnD-DPnD) $(\mathrm{p}<0.05)$; um (TS-SAD) na análise de flechas $(\mathrm{p}<0.05)$; e nove (FSD-ED; BBL-TBL; FSD-BBL; FSD-TBL; ED-BBL; ED-TBL; SAD-SAnD; SAnD-DPnD; GDDPnD) na análise do escore. A análise de Vetor Codificado apresentou que, no total de flechas disparadas nove pares (FSD-ED; GD-PMC; FSD-BBL; FSD-TBL; ED-BBL; ED-TBL; PMC-SAD; SAD-SAnD; SAnD-DPnD) mudaram a moda de fase de coordenação na transição de ajustes posturais, enquanto no desempenho 11 pares (FSDED; BBL-TBL; GD-PMC; TS-SAD; FSD-BBL; FSD-TBL; ED-BBL; ED-TBL; PMCSAD; SAD-SAnD; SAnD-DPnD) evidenciaram tais mudanças. As técnicas apresentadas no presente estudo revelaram parâmetros em comum associados com as sequências temporais de disparo de flechas e com os escores obtidos por arqueiros de elite, isto corrobora com a complexidade da análise do desempenho em atletas de alto rendimento, contudo também colocam a disposição técnicas que ajudam na filtragem e verificação desses parâmetros outrora divergentes.

Palavras-chave: Análise de Vetor Codificado. Índice de co-ativação. Índice de inibição recíproca. Desempenho motor. Variabilidade. Coordenação do movimento. Graus de liberdade. Sistemas Dinâmicos. Hipótese de Ponto de Equilíbrio. 


\begin{abstract}
REISER, Fernando Carvalheiro. Motor control approaches in temporal and performance factors on elite archers. 2019. 130 p. Dissertation (Master in Sciences) School of Arts, Sciences and Humanities, University of São Paulo - São Paulo/SP. 2019. Corrected version
\end{abstract}

Archery is an Olympic sport in which the athletes exhibit several muscular coordination patterns in the arrow shooting, mainly in the clicker falls tempo, making difficult the interpretation of the coordination of archers in what concerns to their score. Thus, some techniques, such as Vector Coding and co-activation/reciprocal inhibition, from Motor Control approaches, can help us to discover what common patterns these archers use when firing and their relation to their obtained performance during the sequential firing of arrows. Aim: evaluate the muscular coordination used by archers of the Brazilian team during an Olympic qualifying simulation, using the electromyographic technique. Eleven archers of the Brazilian team participated; age: $25.2 \pm 4.9$ years, height: $1.72 \pm$ 0.12m, weight: $68.6 \pm 11.8 \mathrm{~kg}$, FITA score: $1258 \pm 43$ points, Qualification: $632 \pm 20$ points, Mean arrow: $8.7 \pm 0.3$ points. The EMG analysis was divided in 0.5 s before and $0.15 \mathrm{~s}$ after the release of the clicker. The RMS value of this twelve-muscle signal was used: drawning arm (dominant side) - FDS (Flexor Digitorum Superficilis); ED (Extensor Digitorum); BB (Biceps Brachii); TBL (Triceps Brachii - Long Head); LD (Latissimus Dorsi) PMC (Pectoralis Major - Clavicular Head); UT (Upper Trapezius); ASD (Anterior Serratus); RA (Rectus Abdominis) and LM (Lumbar Multifidus). Nondominant side (nD) DPnD (Posterior deltoid) and ASnD (Anterior Serratus nondominant side); pairs created (Agonist-Antagonist: FDS-ED, BBL-TBL, LD-PMC, UT-ASD, RA-LM; Distal-Proximal: FDS-BBL, FDS-TBL, ED-BBL, ED-TBL and the pairs glenohumeral-scapular: PMC-SAD, GD-TS, SAD-SAnD, SAnD-DPnD, GDDPnD) for analysis of co-activation/reciprocal inhibition and Vector Coding. Results; eight pairs (FDS-ED, BB-TBL, UT-ASD, FDS-BB, FDS-TBL, ED-TBL, ASD-ASnD, ASnD-DPnD) coactivated differently in blocks transition ( $\mathrm{p}<0.05)$; and 12 (FDS-ED, BB-TBL, LD-PMC, UT-ASD, FDS-BB, FDS-TBL, ED-BB, ED-TBL, PMC-ASD, ASD-ASnD, ASnD-DPnD, LD-DPnD) co-activated differently in the score comparison $(\mathrm{p}<0.05)$. Also, the archers displayed different strategies of reciprocal inhibition in the blocks transition (FDS-ED, BB-TBL, UT-ASD, FDS-BB, FDS-TBL, ED-BBL, EDTBL, ASD-ASnD; ASnD-DPnD) $(\mathrm{p}<0.05)$; one (UT-ASD) in the arrow analysis $(\mathrm{p}<0.05)$; and nine (FDS-ED, BB-TBL, FDS-BBL, FDS-TBL, ED-BBL, ED-TBL, 
ASD-ASnD, ASnD-DPnD, LD-DPnD) in the score analysis. Nine Vector Coding pairs (FDS-BB, FDS-TBL, ED-BB, ED-TBL, PMC-ASD, ASD-ASnD, ASnD- DPnD) changed the coordination phase mode in postural adjustments transition, while 11 pairs (FDS-ED; BB-TBL; LD-PMC; UT-ASD; FDS-BB; FDS-TBL; ED-BB; ED-TBL, PMC-ASD, ASD-ASnD, ASnD-DPnD) showed such changes in score analysis. The techniques presented in the present study revealed common parameters associated with temporal shoot-out and scores obtained by elite archers, dispite the complexity of performance analysis in high-performance athletes.

Keywords: Vector Coding analysis. Co-activation index. Reciprocal inhibition index. Motor performance. Variability. Movement coordination. Degrees-of-freedom. Dynamical Systems Approach. Equilibrium Point Hypothesis. 


\section{LISTA DE FIGURAS}

Figura 1. Ilustração do dispositivo clicker no arco .................................................................. 19

Figura 2. Muybridge Flying Horse de Human and Animal Locomotion .................................... 23

Figura 3. Ciclograma laboral - movimento do ferreiro. ............................................................. 24

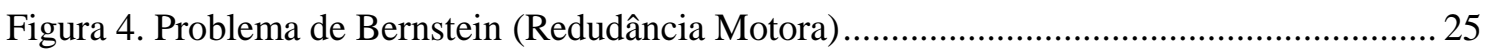

Figura 5. Hipótese de perda de complexidade aplicado a lesão ou doença.................................... 27

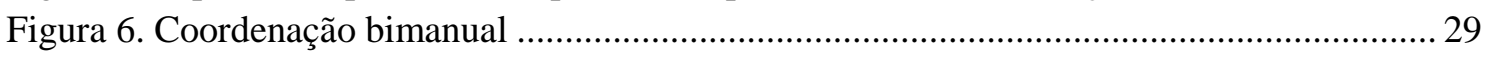

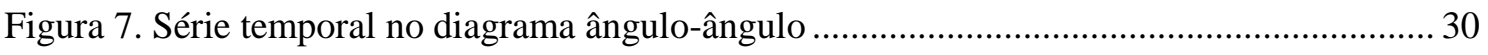

Figura 8. Sequência evolutiva de Análise de Vetor Codificado................................................ 31

Figura 9. Classificação do padrão de coordenação de acordo com análise de Vetor Codificado 33

Figura 10. Sistema de coordenação para análise dos músculos .............................................. 42

Figura 11. Análise de Vetor Codificado das frequências, por percentuais, do índice FSD-ED .. 73

Figura 12. Análise de Vetor Codificado das frequências, por percentuais, do índice BBL-TBL.

Figura 13. Análise de Vetor Codificado das frequências, por percentuais, do índice GD-PMC. 75 Figura 14. Análise de Vetor Codificado das frequências, por percentuais, do índice TS-SAD. 77 Figura 15. Análise de Vetor Codificado das frequências, por percentuais, do índice RA-ML... 78 Figura 16. Análise de Vetor Codificado das frequências, por percentuais, do índice FSD-BBL.

Figura 17. Análise de Vetor Codificado das frequências, por percentuais, do índice FSD-TBL.

Figura 18. Análise de Vetor Codificado das frequências, por percentuais, do índice ED-BBL. 83

Figura 19. Análise de Vetor Codificado das frequências, por percentuais, do índice ED-TBL.. 85

Figura 20. Análise de Vetor Codificado das frequências, por percentuais, do índice PMC-SAD.

Figura 21. Análise de Vetor Codificado das frequências, por percentuais, do índice GD-TS .... 88

Figura 22. Análise de Vetor Codificado das frequências, por percentuais, do índice SAD-SAnD

Figura 23. Análise de Vetor Codificado das frequências, por percentuais, do índice DPnD-

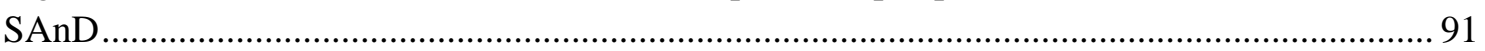

Figura 24. Análise de Vetor Codificado das frequências, por percentuais, do índice GD-DPnD92 


\section{LISTA DE TABELAS}

Tabela 1. Pares de músculos agonistas-antagonistas analisados no presente estudo ................... 40

Tabela 2. Indíce com par distal-proximal das articulações do punho e do cotovelo ..................... 41

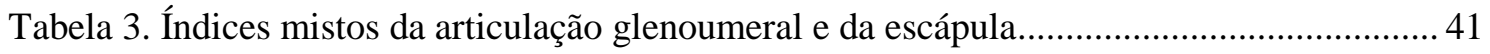

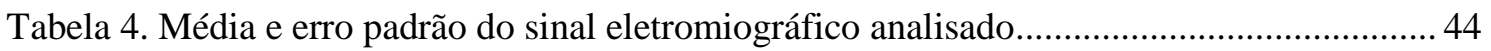

Tabela 5. Co-ativação do par FSD-ED com Média e EP .............................................................. 45

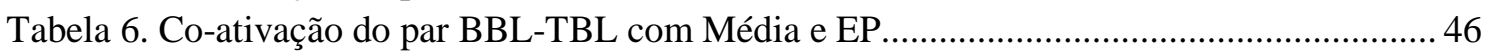

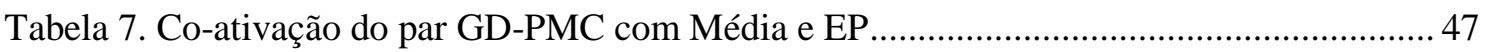

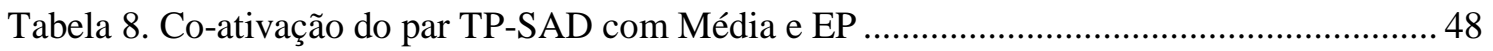

Tabela 9. Co-ativação do par RA-ML com Média e EP .............................................................. 49

Tabela 10. Co-ativação do par FSD-BBL com Média e EP........................................................... 50

Tabela 11. Co-ativação do par FSD-TBL com Média e EP ....................................................... 51

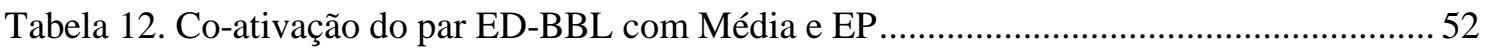

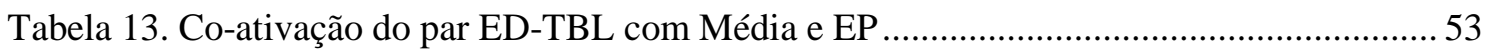

Tabela 14. Co-ativação do par PMC-SAD com Média e EP ....................................................... 54

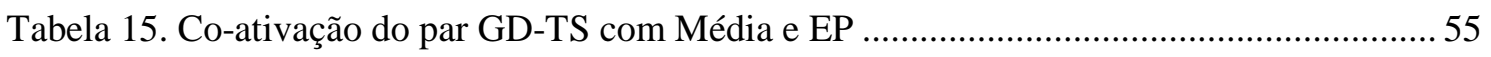

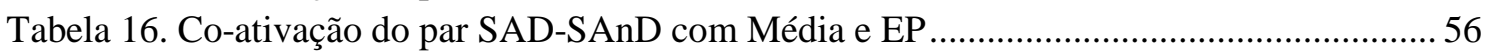

Tabela 17. Co-ativação do par SAnD-DPnD com Média e EP ..................................................... 57

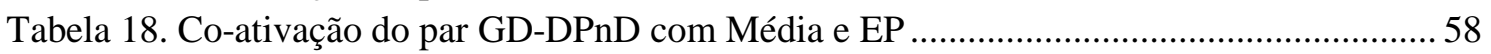

Tabela 19. Inibição reciproca do par FSD-ED com Média e EP ................................................. 59

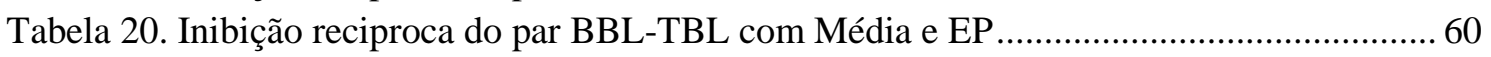

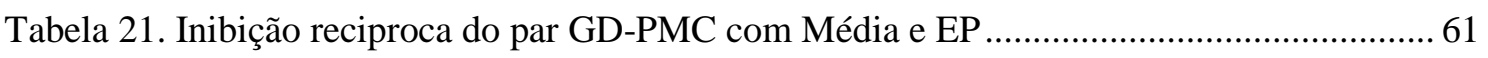

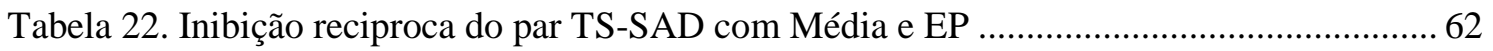

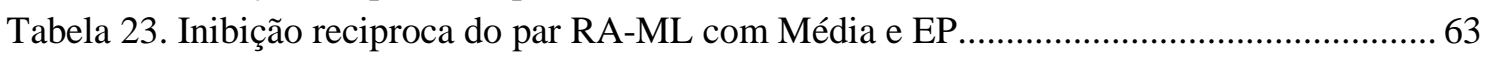

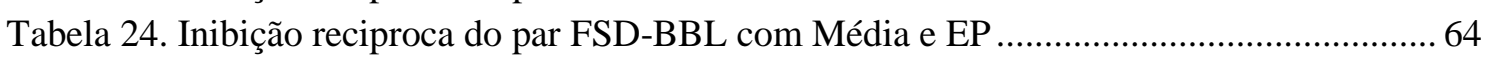

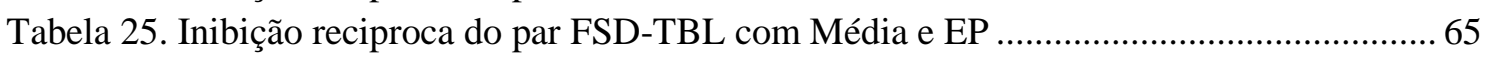

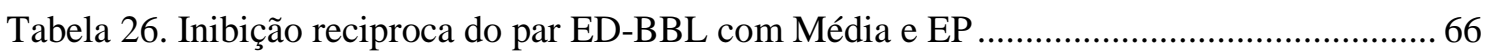

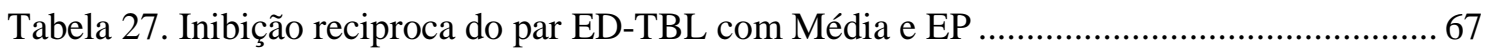

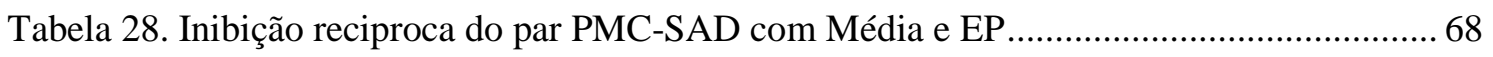

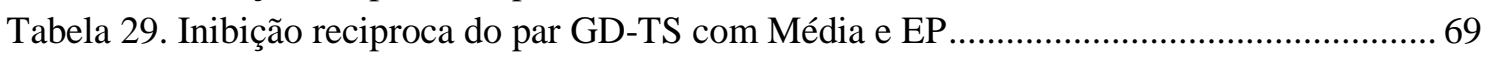

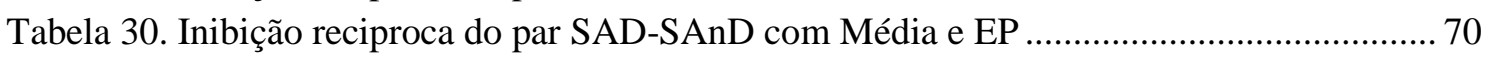

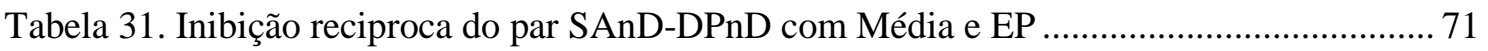

Tabela 32. Inibição reciproca do par GD-DPnD com Média e EP ............................................ 72

Tabela 33. Análise de Vetor Codificado - frequências, em percentis, por escore (a moda está

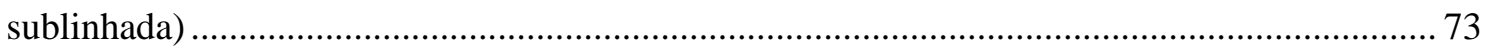

Tabela 34. Moda da fase de coordenação por escore - resumida ................................................. 73

Tabela 35. Análise de Vetor Codificado - frequências, em percentis, por escore (a moda está

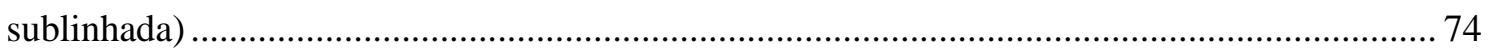

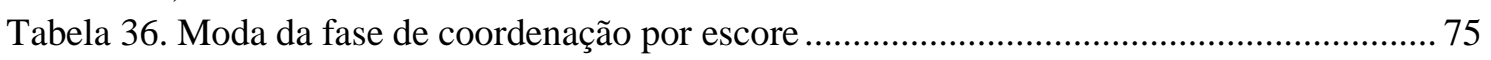

Tabela 37. Análise de Vetor Codificado - frequências, em percentis, por escore (a moda está

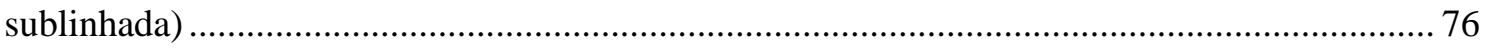

Tabela 38. Moda da fase de coordenação por escore …………………………........................ 76

Tabela 39. Análise de Vetor Codificado - frequências, em percentis, por escore (a moda está

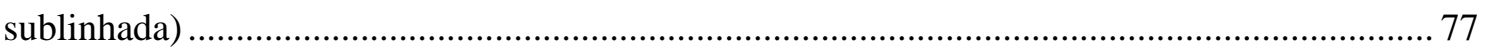

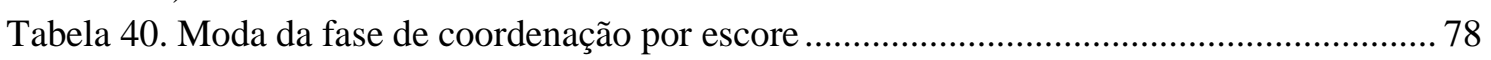

Tabela 41. Análise de Vetor Codificado - frequências, em percentis, por escore (a moda está

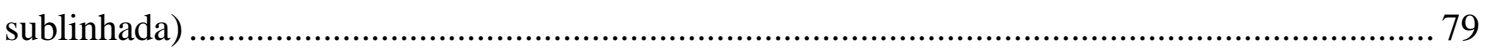

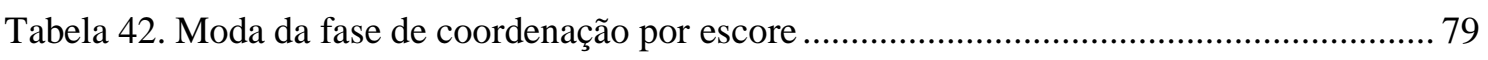


Tabela 43. Análise de Vetor Codificado - frequências, em percentis, por escore (a moda está sublinhada)

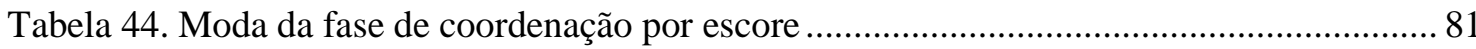

Tabela 45. Análise de Vetor Codificado - frequências, em percentis, por escore (a moda está

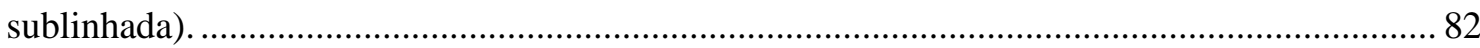

Tabela 46. Moda da fase de coordenação por escore resumida …............................................. 83

Tabela 47. Análise de Vetor Codificado - frequências, em percentis, por escore (a moda está

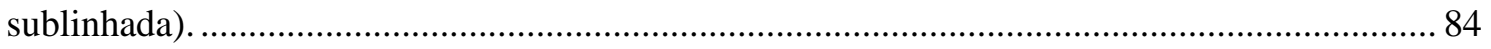

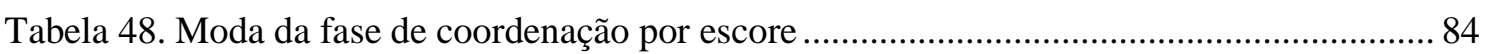

Tabela 49. Análise de Vetor Codificado - frequências, em percentis, por escore (a moda está

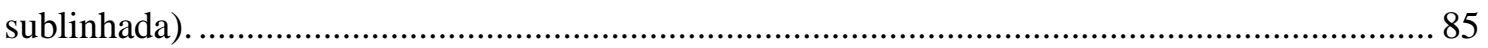

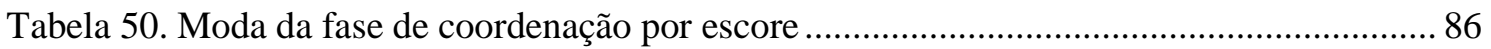

Tabela 51. Análise de Vetor Codificado - frequências, em percentis, por escore (a moda está

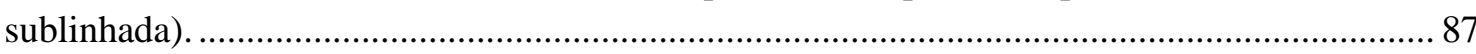

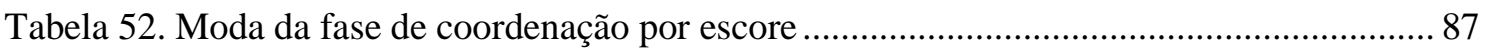

Tabela 53. Análise de Vetor Codificado - frequências, em percentis, por escore (a moda está

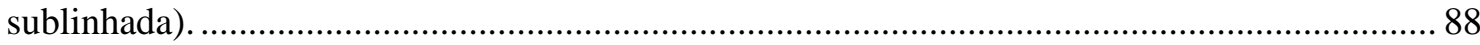

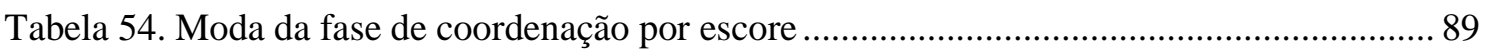

Tabela 55. Análise de Vetor Codificado - frequências, em percentis, por escore (a moda está

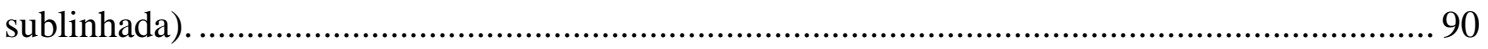

Tabela 56. Moda da fase de coordenação por escore ………………......................................... 90

Tabela 57. Análise de Vetor Codificado - frequências, em percentis, por escore (a moda está

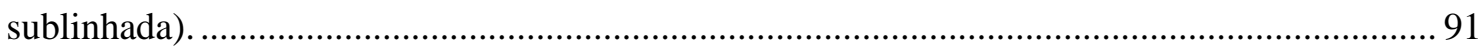

Tabela 58. Moda da fase de coordenação por escore ............................................................... 92

Tabela 59. Análise de Vetor Codificado - frequências, em percentis, por escore (a moda está

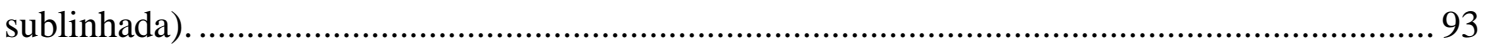

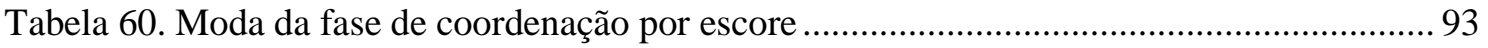

Tabela 61. Resumo dos resultados encontrados em todos os índices analisados ......................... 94 


\section{LISTA DE SIGLAS}

APA - Ajuste Postural Antecipatório

APC - Ajuste Postural Compensatório

AVC - Análise de Vetor Codificado

BBL - músculo Bíceps Braquial

C- Comando de co-ativação

DP - músculo Deltóide Posterior

DPnD - músculo Deltóide Posterior - lado não-dominante

D - lado dominante - que traciona a corda do arco

ED - músculo Extensor dos Dedos

EP - Erro Padrão

EMG - Eletromiografia

EMGnorm - Sinal eletromiográfico normalizado

FSD - músculo Flexor Superficial dos Dedos

GD - músculo Grande Dorsal / Latíssimo do Dorso

HPT - Hipótese do Ponto de Equílibrio

ML - músculo Multífido Lombar

$\mathrm{nD}$ - lado não-dominante - que segura o arco

PMC - músculo Peitoral Maior - cabeça/porção Clavicular

$\mathrm{R}$ - Comando de inibição recíproca

RA - músculo Reto Abdominal

RMS - Raiz Quadrática Média (Root Mean Square)

SAD - músculo Serrátil Anterior - lado dominante

SAnD - músculo Serrátil Anterior - lado não-dominante

SD - Sistemas Dinâmicos

SE - Standard Error (Erro Padrão)

SNC - Sistema Nervoso Central

TBL - músculo Tríceps Braquial - cabeça/porção Longa

TS - músculo Trapézio - porção Superior/descendente

VC - Vector Coding (Vetor Codificado) 


\section{SUMÁRIO}

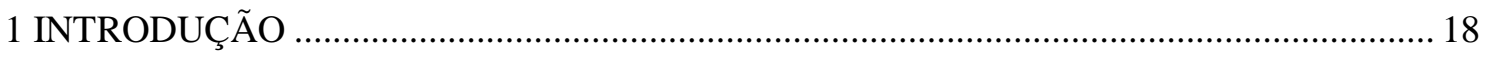

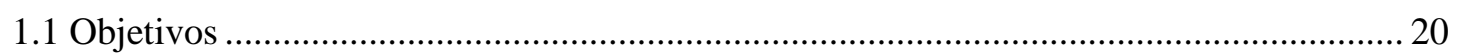

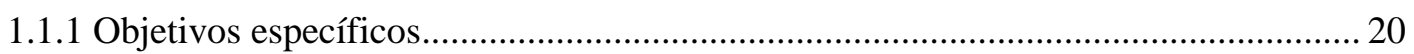

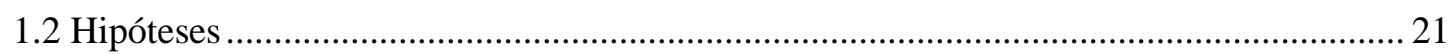

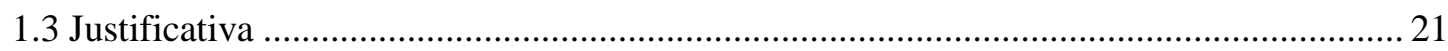

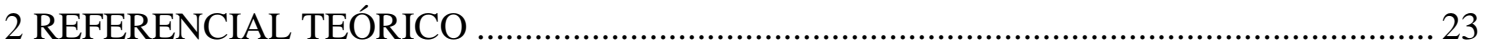

2.1 Variabilidade e Coordenação no desempenho humano....................................................... 26

2.1.1 Sistemas Dinâmicos (Dynamical Systems Approach) ................................................. 29

2.1.2 Hipótese do Ponto de Equilíbrio .................................................................................. 34

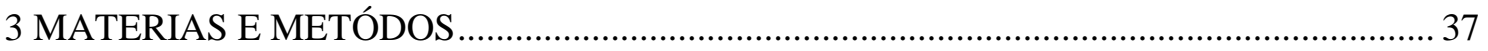

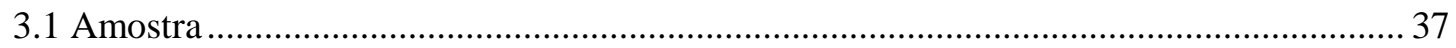

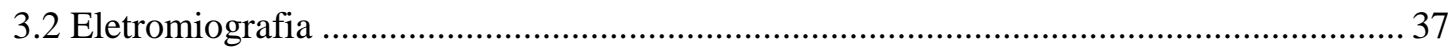

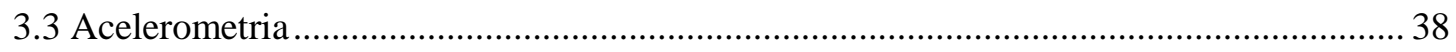

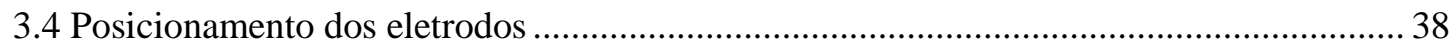

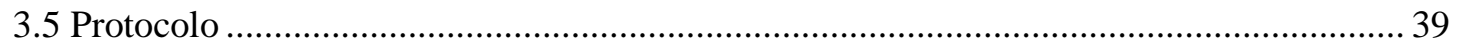

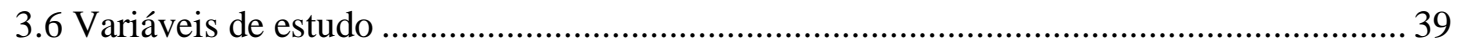

3.7 Construção dos Índices de Co-ativação e de Inibição Recíproca......................................... 40

3.8 Análise de Vetor Codificado ................................................................................................ 41

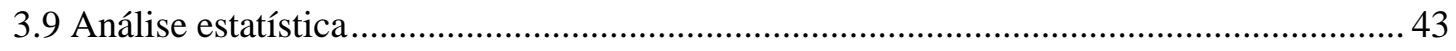

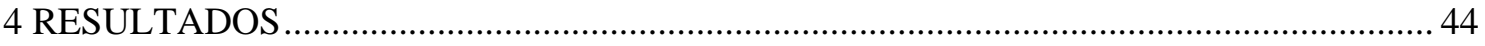

4.1 Resultados descritivos de pontuação e eletromiografia........................................................ 44

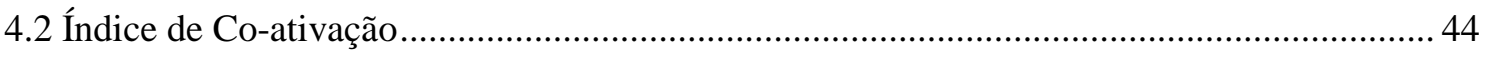

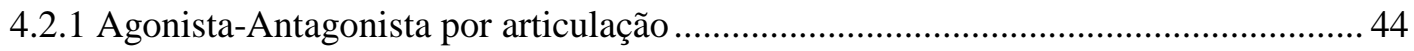

4.2.2 Distal-Proximal (Punho e cotovelo) ............................................................................. 49

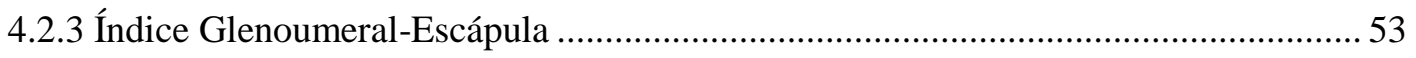

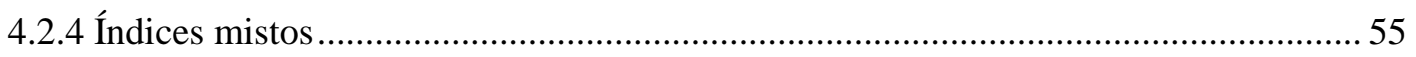

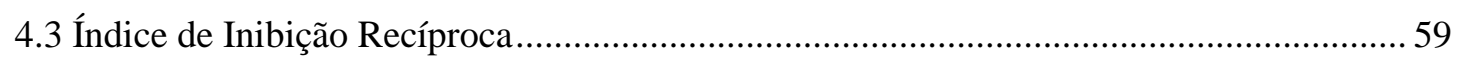

4.3.1 Agonista-Antagonista por articulação …………………………………….............. 59

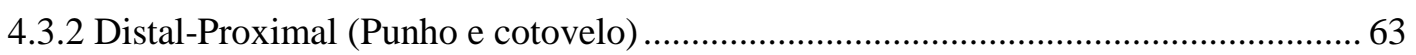

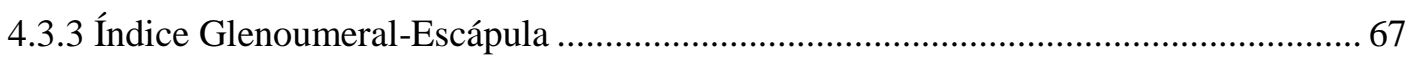

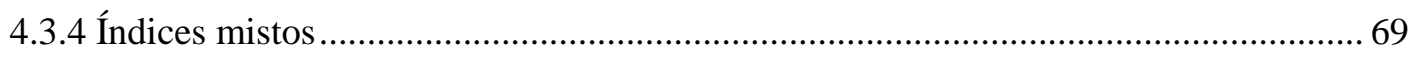

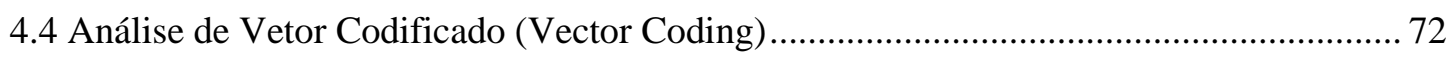

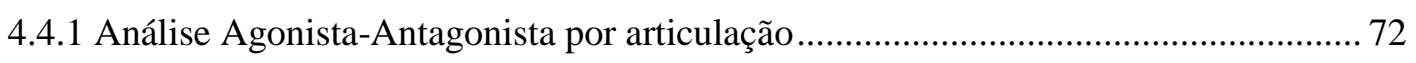

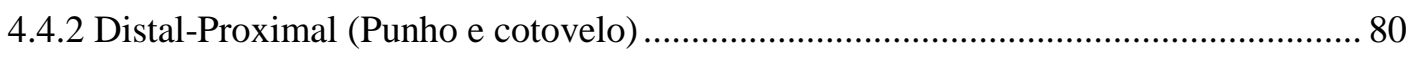

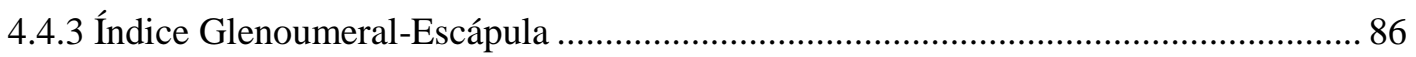




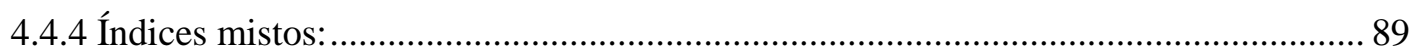

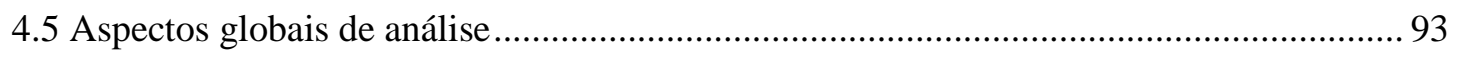

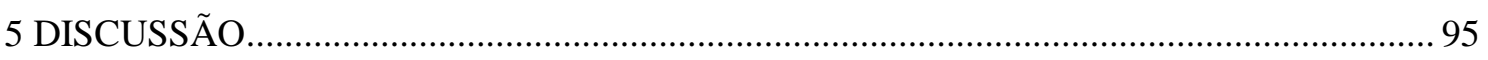

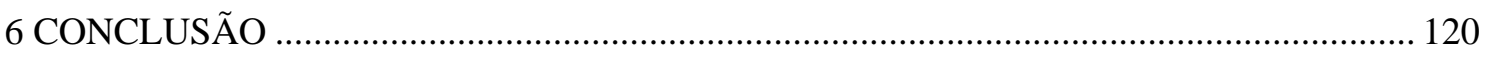

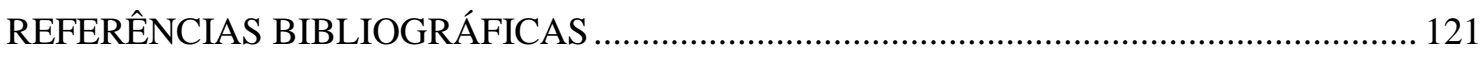

APÊNDICE I - TERMO DE CONSENTIMENTO LIVRE E ESCLARECIDO........................ 127

APÊNDICE II - RESUMO APRESENTADO EM CONGRESSO - COBEC.......................... 130

APÊNDICE III - RESUMO APRESENTADO EM CONGRESSO - ICSEMIS ....................... 132 


\section{INTRODUÇÃO}

A análise do desempenho esportivo é um objeto de interesse dos pesquisadores do movimento humano e treinadores esportivos, principalmente pela complexidade e a variabilidade na interpretação de resultados produzidos por atletas de alto rendimento. Por exemplo, o mais rápido velocista de $100 \mathrm{~m}$ e $200 \mathrm{~m}$ rasos do mundo possui assimetria na distribuição de sobrecarga em membros inferiores na sua corrida, que gera até $13 \%$ a mais de impacto em um membro do que no outro, e na prova que este mesmo atleta quebrou o recorde mundial dos $100 \mathrm{~m}$, a sua largada foi a terceira pior entre os finalistas da prova (UDOFA et al., 2017; WEYAND, 2017; GRAUBNER, NIXDORF, 2011). Morris, Bartlett, Fowler et al., (1997) avaliaram atletas na final do lançamento de dardos durante o 1995 World Athletics Championships, com foco nas análises de membros superiores na velocidade de lançamento. O medalhista de prata atingiu maior velocidade angular no ombro - o que sugere um parâmetro de lançamento que utiliza maior flexão horizontal e extensão da articulação glenoumeral produzindo um deslocamento mais linear do dardo, já o campeão da prova utilizou maior rotação interna desta mesma articulação combinada com velocidade angular na extensão do cotovelo quase $20 \%$ maior que os demais competidores, os outros finalistas também utilizaram coordenações intersegmentares heterogêneas no lançamento. No tiro com arco a análise dos medalhistas no 44th World Outdoor Target Archery Championships evidenciou que eles realizavam a preparação do tiro em menor tempo que os demais atletas, sugerindo que os medalhistas possuem a capacidade de realizar ajustes posturais e estratégicos mais rapidamente, ou seja, tanto em esportes de tendência mais dinâmica como também estática existem parâmetros particulares relativos aos melhores desempenhos obtidos (TAKAI; KUBO; ARAKI, 2012).

O arqueiro leva em média no máximo $200 \mathrm{~ms}$ para reagir ao estímulo sensorial do clicker (Figura 1) e efetuar o disparo (MARTIN; SILER; HOFFMAN, 1990), este dispositivo serve para padronizar a puxada da corda individualmente, e estabilizar o tiro no ínicio do lançamento. Verifica-se diferença evidente de atividade muscular na comparação de atletas com os mais variados níveis de habilidade. Ertan et al. (2003) identificaram que os mais habilidosos reagem mais rapidamente com a ativação muscular de extensores dos dedos 100ms após a queda deste dispositivo; enquanto que iniciantes e não-arqueiros possuem maior hesitação, utilizando o dobro e o triplo do tempo respectivamente. Existem casos de completa inibição recíproca após a queda do clicker, Ertan et al. (2011) notaram esta estratégia em uma arqueira de elite - entre as dez melhores do mundo - sugerindo a hipótese que a própria força da corda nos dedos 
seria suficiente para realizar sua extensão. Recentemente, Simsek et al. (2014; 2018) observaram que durante a puxada da corda, arqueiros de elite utilizam menos os músculos distais (antebraço), e mais os proximais (glenoumeral), e axiais (trapézio), enquanto os de nível médio e iniciantes ativam mais os músculos do antebraço. Esta prioridade de coordenação face a músculos proximais e axiais sugere que o atleta de alto rendimento pretende minimizar as oscilações horizontais do arco, além de uma provável interação mais eficiente entre atleta-arco em comparação à aqueles de menor desempenho.

Figura 1. Ilustração do dispositivo clicker no arco
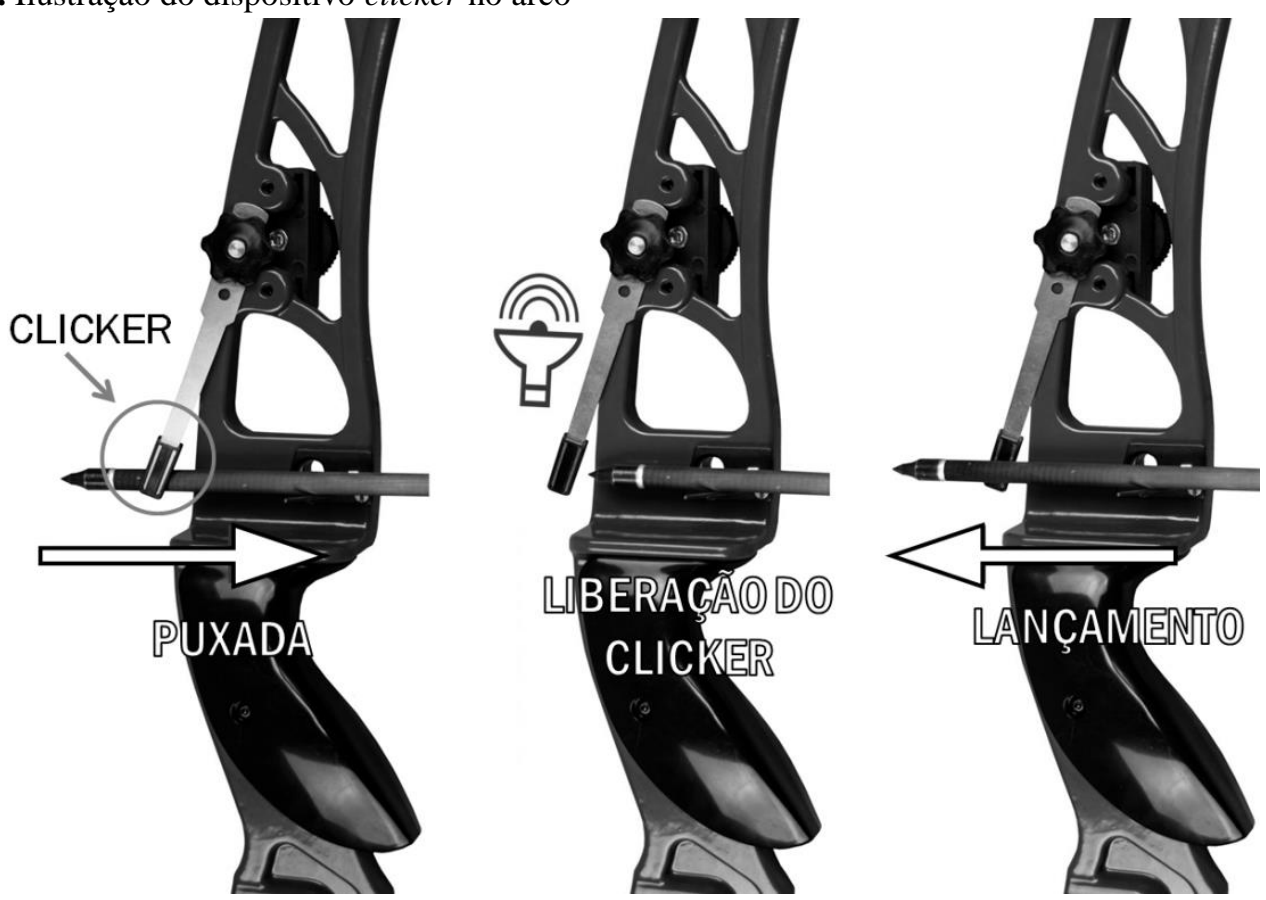

Fonte: Adaptação de Fernando Carvalheiro Reiser (2019) retirado de Apex Hunting (2019)

Legenda: O dispostivo do clicker (demonstrado no círculo cinza) serve para padronizar a puxada do arqueiro, na puxada final o arqueiro deve liberar o clicker causando um estímulo sensorial e audível, e então iniciar imediatamente o lançamento (disparo da flecha).

Existem mecanismos individuais de atividade muscular entre os atletas de ponta, principalmente para a sua pontuação obtida, como evidencia Suwarganda et al. (2012). Eles observaram estratégias distintas nos músculos tríceps braquial, deltóide e trapézio. Em um dos casos, a pontuação de um arqueiro estava relacionada com a atividade muscular do trapézio; enquanto noutro a pontuação mais elevada correlacionou-se à atividade de tríceps braquial do lado que tracionava a flecha. Tinazci (2011) observou que a média eletromiográfica dos flexores e extensores do antebraço, trapézio superior e deltóide anterior foram menores conforme os atletas obtinham pontuações mais elevadas. Além disso, o deslocamento médio-lateral do COP teve relação negativa com a pontuação, enquanto que o deslocamento anteroposterior do COP foi mais variável e individual entre os atletas analisados. 
Estes estudos evidenciam a complexidade para a análise do desempenho motor, e dificuldade para a busca por parâmetros generalizados de movimento, contudo existem mecanismos para avaliar a coordenação do movimento humano, dentro do estudo do Controle Motor, que permitem uma melhor compreensão destes mecanismos de variabilidade no desempenho motor. De acordo com Guigon (2011), neste nível de análise encontramos duas abordagens, com certos elementos antagônicos, donde as técnicas provenientes podem dar mais subsidios para análise do movimento humano. A abordagem da Hipótese do Ponto de Equilíbrio, por exemplo, possui certas técnicas para averiguação da coordenação muscular através da análise de co-ativação e de inibição recíproca que permitem a verificação das estratégias predominantes utilizadas no Tiro com Arco e sua relação com desempenho tiro-a-tiro e nos escores obtidos, como por exemplo, no que tange as coordenações musculares entre Flexores Superficiais dos Dedos e Extensores dos Dedos que evidenciam resultados conflitantes na literatura. Já nos Sistemas Dinâmicos, podemos verificar a coordenação muscular, por exemplo, através da técnica de Análise de Vetor Codificado, que permite verificar a sincronia entre dois músculos numa articulação para análise também de co-ativações, ou inibições e a frequência destas ocorrências, e também a sua relação com desempenho tiro-a-tiro e nos escores obtidos.

Pela perspectiva eletromiográfica, a pergunta de pesquisa desta dissertação de mestrado é como estas abordagens podem oferecer novos subsídios ao estudo do desempenho humano para o tiro com arco, principalmente face aos comandos realizados nos ajustes posturais, e nas estratégias de coordenação utilizadas por arqueiros de alto rendimento durante uma simulação de uma prova olímpica, composta por 12 blocos de 6 flechas lançadas a $70 \mathrm{~m}$ do alvo.

\subsection{Objetivos}

O objetivo principal do presente estudo foi o de avaliar o desempenho de arqueiros da seleção brasileira por meio de diferentes abordagens de controle motor na atividade eletromiográfica.

\subsubsection{Objetivos específicos}

Descrever e analisar os mecanismos temporais nos Ajustes Posturais Antecipatórios (APA) - antes da queda do clicker, e Compensatórios (APC) - após a queda do clicker. Mecanismos estes de co-ativação e inibição reciproca, e de fase de coordenação nos músculos de controle distal (punho), distal-proximal (cotovelo), proximal (glenoumeral) e proximal-axial (escápula e tronco) em arqueiros de elite. 
Descrever e analisar os mecanismos de desempenho (pontuação) no APA e APC - de co-ativação e inibição reciproca, e de fase de coordenação nos músculos de controle distal (punho), distal-proximal (cotovelo), proximal (glenoumeral) e proximal-axial (escápula e tronco) em arqueiros de elite.

\subsection{Hipóteses}

\section{Hipótese nula}

$\mathrm{H}_{0}$ : Os arqueiros atirarão as flechas com mecanismos de co-ativação nos ajustes posturais serão os mesmos para as análises dos blocos, flechas e de pontuação. De igual modo os mecanismos de inibição recíproca nos ajustes posturais serão os mesmos para as análises dos blocos, flechas e de pontuação. Os arqueiros atirarão as flechas utilizando um tipo de fase de coordenação nos ajustes posturais na análise total de flechas disparadas e de pontuação.

Hipótese alternativa

$\mathrm{H}_{\mathrm{A}}$ : Os arqueiros atirarão as flechas com mecanismos de co-ativação nos ajustes posturais diferentes para análise dos blocos, flechas e de pontuação. De igual modo os mecanismos de inibição recíproca nos ajustes posturais serão diferentes para análise dos blocos, flechas e de pontuação. Os arqueiros atirarão as flechas utilizando mais de um tipo de fase de coordenação nos ajustes posturais na análise total de flechas disparadas e de pontuação.

\subsection{Justificativa}

Foram utilizadas para averiguar os mecanismos temporais e de pontuação nos atletas a análise dos índices de co-ativação e inibição recíproca, e a análise de Vetor Codificado, esta foi utilizada como um indicativo de desempenho entre atletas iniciantes e no salto triplo, identificando uma curva em $U$ na relação dos acoplamentos segmentares de atletas mais iniciantes a intermediários e avançados (WILSON et al.,2008), enquanto aquelas primeiras foram ferramentas utilizadas em diversos contextos na literatura, como por exemplo, na análise da postura em pé, cujos resultados evidenciaram maiores mecanismos de co-ativação dos músculos de controle postural que de inibição recíproca (SLIJPER; LATASH, 2000). No presente estudo foram utilizadas as técnicas referidas para identificar em arqueiros de elite as diversas estratégias de coordenação muscular e averiguar quais delas podem ser moduladas em maior e menor frequência conforme a variação de bloco-a-bloco, flechas e escore 
obtido. A modalidade escolhida permite averiguação destes parâmetros pela quantidade de tiros disparos, permitindo a análise repetitiva dos mesmos, e a curta duração onde estas modulações ocorrem. Entre a queda do clicker e efetivo disparo da flecha temos um período de até $500 \mathrm{~ms}$ onde algumas estratégias de coordenação, principalmente entre flexores e extensores do punho e dos dedos ocorrem (ERTAN et al., 2003, 2011; MARTIN; SILER; HOFFMAN, 1990; MARTIN; HEISE, 1992), tornando-se interessante para a utilização destas ferramentas. Destaca-se a verificação das estratégias em demais músculos de membros superiores e tronco que também parecem ser modulados antes e após a queda do clicker (SHINOHARA, URABE, 2017; NISHIZONO et al., 1987; KOLAYIŞ, ERTAN, 2016), para uma análise mais aprofundada do fenômeno. A utilização destas técnicas tem potência de aplicação em outras análises de desempenho motor, podendo dar novos subsídios a estas análises em âmbito do esportivo ou reabilitativo. 


\section{REFERENCIAL TEÓRICO}

No príncipio da técnica de cinemetria, Eadweard Muybridge obteve uma análise peculiar a respeito da fase aérea do movimento de cavalos (FIGURA 2), que posteriormente culminou nos fatores coordenativos que distinguiam a corrida da marcha em seres humanos (LESLIE, 2001), o filósofo Aristóteles, nos primórdios da investigação biológica, também observara aspectos coordenativos que diferenciavam os vários gestos motores do homem. A sistematização destes aspectos coordenativos no movimento humano ocorre somente com o cientista Bernstein (1967). Ele define a coordenação do movimento humano como o domínio dos múltiplos graus de liberdade (coordenadas independentes que são utilizadas para especificar um corpo, sistema ou posição) envolvidos na execução de um determinado movimento, que possibilita tornar as articulações, os segmentos, os músculos e as unidades motoras um sistema controlável (BERNSTEIN, 1967; BERNSTEIN; LATASH; \& TURVEY, 1996).

Figura 2. Muybridge Flying Horse de Human and Animal Locomotion

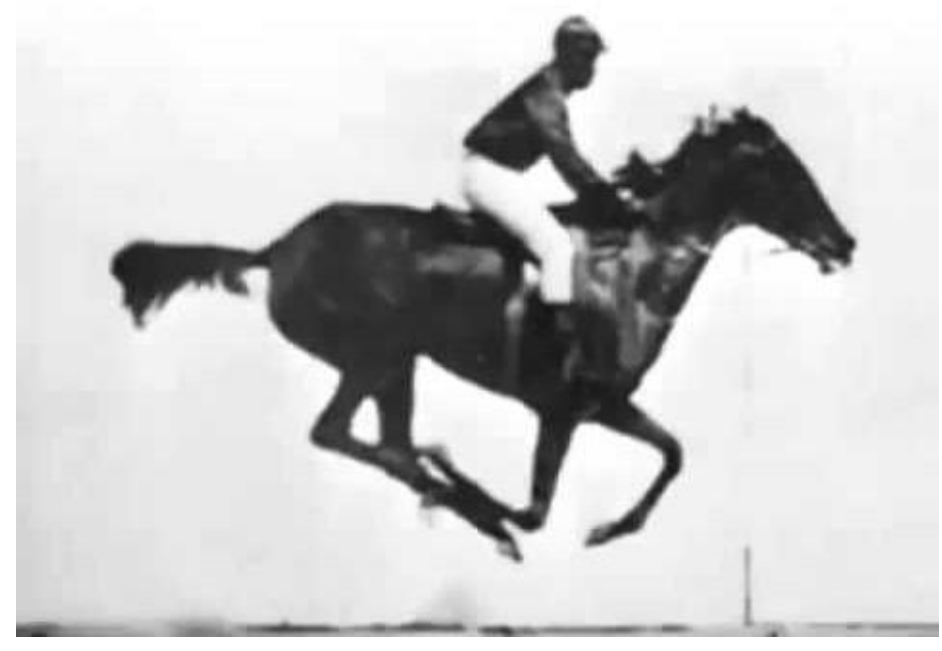

Fonte: Leslie (2001) adaptado de Muybridge (1878)

A constatação de Bernstein surge da sua investigação com ferreiros (FIGURA 3), quando os filma na sua prática profissional - equipados com bulbos elétricos em diferentes partes corporais - batendo o martelo no cinzel. Uma das principais conclusões deste trabalho foi a de que, esses ferreiros utilizavam trajetórias articulares variáveis durante a tarefa - após repetidas vezes - mesmo com o ponto final da tarefa invariável. Essa variabilidade, que é uma característica do nosso sistema para obter desempenho motor por meio de diferentes soluções ao longo do tempo ou durante repetidas tentativas (LATASH, 2008). Foi posteriormente observada tanto em outras profissões laborais, e artísticas como em esportistas de alto rendimento, além disso, nós possuímos 
mais graus de liberdade que o necessário para atingir determina meta na tarefa realizada, o que também confere uma determinada redundância de todo nosso aparelho locomotor (BERNSTEIN, 1967; LATASH, ZATSIORSKY, 2015; BERNSTEIN, LATASH, TURVEY, 1996).

Figura 3. Ciclograma laboral - movimento do ferreiro.

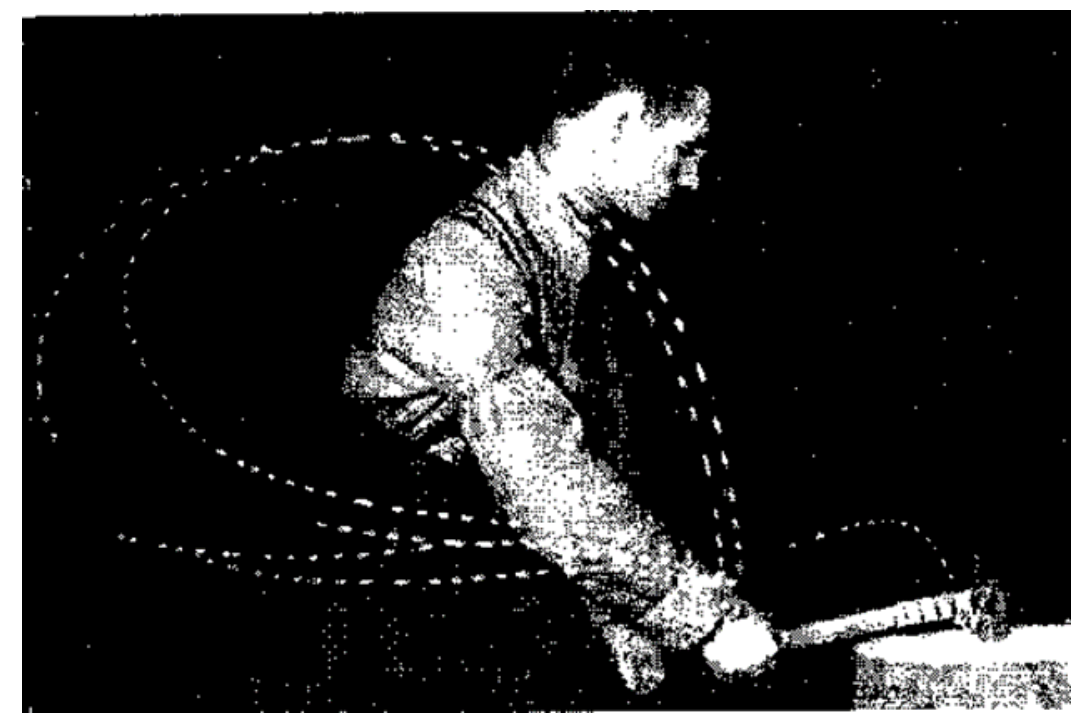

Fonte: Bernstein (1967) adaptado de Aleksei Gastev.

A partir das evidências de redundância, Bernstein formula o questionamento de como o Sistema Nervoso Central (SNC) seleciona a resolução do problema motor dentro de uma infinidade de possibilidades (problema dos graus de liberdade ou "Problema de Bernstein"), por se tratar justamente dum sistema redundante - múltiplos agentes permitem a flexibilização do movimento - em comparação com os seus restritores (agentes que não permitem a liberdade de movimento) - fato que confere um nível de variabilidade e complexidade ao sistema (BERNSTEIN, 1967). A redundância motora (problema dos graus de liberdade) indica que existe um maior número de soluções para as tarefas motoras do que o número de limitadores (restritores, do tipo, biomecânicos, morfológicos e ambientais) quando comparamos com as Variáveis Elementares (graus de liberdade); estas são variáveis que podem ser potencialmente modificadas sem a modificação de outras no mesmo nível. É necessário esclarecer os termos abundância e redundância motora; Bernstein usa “избыточность” (izbytochnost) da raiz избыток (izbtok) que dependendo do contexto pode ser interpretado tanto como redundância ou abundância sem a perda do significado (GELFAND, LATASH, 2002).

A variabilidade aceitável ou prolífica da execução de tarefas motoras se situa entre dois limites, a variabilidade acima do limite superior indica que o sistema está 
instável e sensível a perturbações e a variabilidade abaixo do limite inferior indica que o movimento está estereotipado, com pouca complexidade, com menor capacidade de adaptação à perturbações no sistema (STERGIOU; HARBOURNE; CAVANAUGH, 2006; GLAZIER et al., 2006; NEWELL; JAMES, 2008). O estudo da variabilidade motora, recentemente, tem sido fundamental para entender as habilidades esportivas e aspectos reabilitativos, onde a falta ou perda de variabilidade e complexidade do aparato locomotor associa-se com desempenhos insuficientes ou com a presença de lesão (BARTLETT, WHEAT, ROBINS, 2007; MOSELEY, HODGES, 2006).

A partir de uma série de inferências semelhantes, Bernstein cunha a habilidade do SNC de escolher padrões de movimento específicos como "eliminação dos graus de liberdade redundantes". Pode-se adicionar que o êxito da escolha em cada tentativa possui características como conjectura Guigon (2011). É necessário lidar com as seguintes capacidades: atingir o objetivo com menor erro possível e gasto energético (escolher o melhor padrão de movimento para problema dos graus de liberdade), e defrontar com as perturbações (problema da variabilidade) determinísticas (mudança de objetivo) e estocásticas (ruído no comando motor) do sistema (FIGURA 4).

Figura 4. Problema de Bernstein (Redudância Motora)

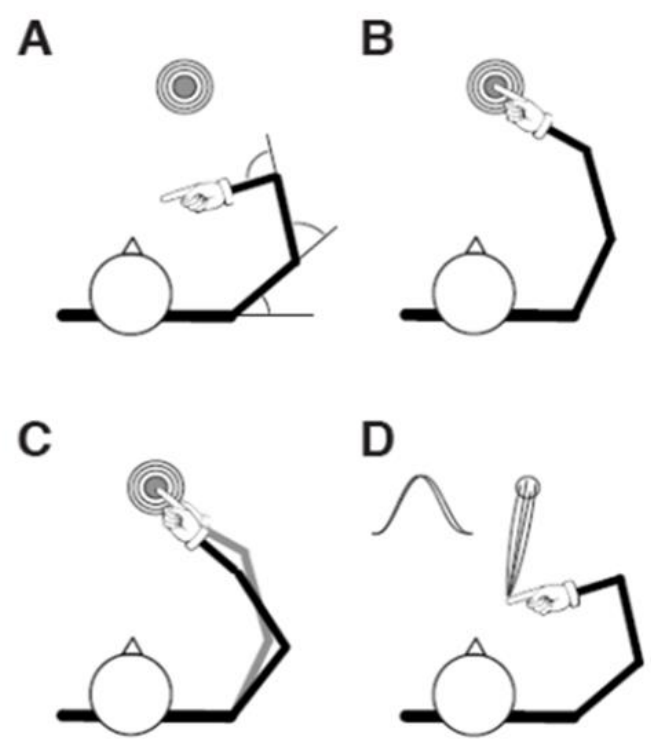

Fonte: Guigon (2011)

Legenda A: Movimento uniplanar de alcance com um braço redundante (três graus de liberdade); B: O movimento do braço bem-sucedido atinge a meta; $\mathrm{C}$ : Dois movimentos sucessivos que atingem a meta com características espaço-temporais diferentes. D: Várias tentativas bem-sucedidas com características espaço-temporais diferentes, com perfis de velocidade inseridos. Adapatado de Guigon (2008).

A partir de todas estas características, podemos destacar duas abordagens para a resolução do "Problema de Bernstein": a otimização, na qual um controlador central encontra a solução do problema cada vez que ele se apresenta, e a abundância, o sistema 
não utiliza uma só solução possível, onde o controlador facilita um grupo de soluções aceitáveis produzindo um sistema flexível de acordo com as características da tarefa, sem o sistema eliminar qualquer grau de liberdade (LATASH, 2008; GELFAND, LATASH, 2002). Pode-se destacar outros aspectos inerentes ao controle como paradoxo movimento/postura, além de o desenvolvimento de simplificações para explanar o controle motor em vários níveis de complexidade. Neste paradoxo os processos que são responsáveis pelo controle postural não parecem interferir diretamente no controle de movimento (Oster \& Feldman, 2003). Reforça-se que abordagem de otimização parte da permissa que o controle de múltiplos graus de liberdade é otimizado pela magnitude da função custo da tarefa (minimizando ou maximizando a mesma). O custo da função é dado pelas variáveis elementares que são minimizadas ou maximizadas pelo controlador (fadiga, solavanco, espaço, tempo e mudança de torque mínimos) diretamente, assumindo o custo e as variáveis elemanteres baseada nos restritores da tarefa ou inversamente, utilizando observações prévias para tal custo.

As abordagens, simplificações e demais conjecturas teóricas propostas a partir das evidências de Bernstein (1967), e como a coordenação emerge para controlar o movimento, necessitam de acordo com Guigon (2011), ser capazes de lidar com os princípios da solução ótima (solução para o problema de Bernstein que gera movimentos realistas em face de redundância espacial, temporal, cinemática e muscular, TODOROV \& JORDAN, 2002), eficácia, feedback e esforço (nos modelos de controle motor, o esforço de um movimento é o produto da amplitude e duração do evento analisado, GUIGON, 2011). Os níveis de análise de cada uma destas conjecturas lidará com essas premissas em diferentes aspectos no sistema cérebro-corpo; 1) a nível de biomecânica e músculo, 2) a nível neuromuscular (conceitos de Sinergia e Eliminação), 3) a nível de controle (relacionado com os processos que convertem os objetivos da tarefa em ativações musculares - destacam-se as Teorias de Ponto de Equilíbrio e dos Sistemas Dinâmicos) e 4) a nível funcional (Planejamento-Execução, e Análise de Postura-Movimento). Destaca-se a importância das abordagens no nível de controle, no estudo da variabilidade e coordenação do movimento, que terá maior relevância para o desenvolvimento do atual trabalho com a exposição de técnicas que emergem delas e suas implicações no desempenho esportivo e humano de modo geral.

\subsection{Variabilidade e Coordenação no desempenho humano}

A presença de múltiplos graus de liberdade na coordenação do movimento humano é fonte potencial de variabilidade, como o ruído no sistema, que seria 
potencialmente prejudicial ao desempenho humano e tratada como fonte de erro. Apesar de possuir esses aspectos, a melhor compreensão dela só foi obtida a partir dos trabalhos de Bernstein (LATASH, TURVEY, BERNSTEIN, 1996), e ao contrário da perspectiva de que níveis elevados de desempenho motor estariam associados com menores níveis de variabilidade, o que ocorre casualmente é o oposto. A perda da variabilidade e a redução dos graus de liberdade parecem estar associados com desempenhos insuficientes. A complexidade do sistema diminui, logo a eficácia da ação motora é menor. Isto é evidente na comparação de habilidades esportivas e na presença de lesões (FIGURA 5) (LIPSITZ, 2002).

Figura 5. Hipótese de perda de complexidade aplicado a lesão ou doença

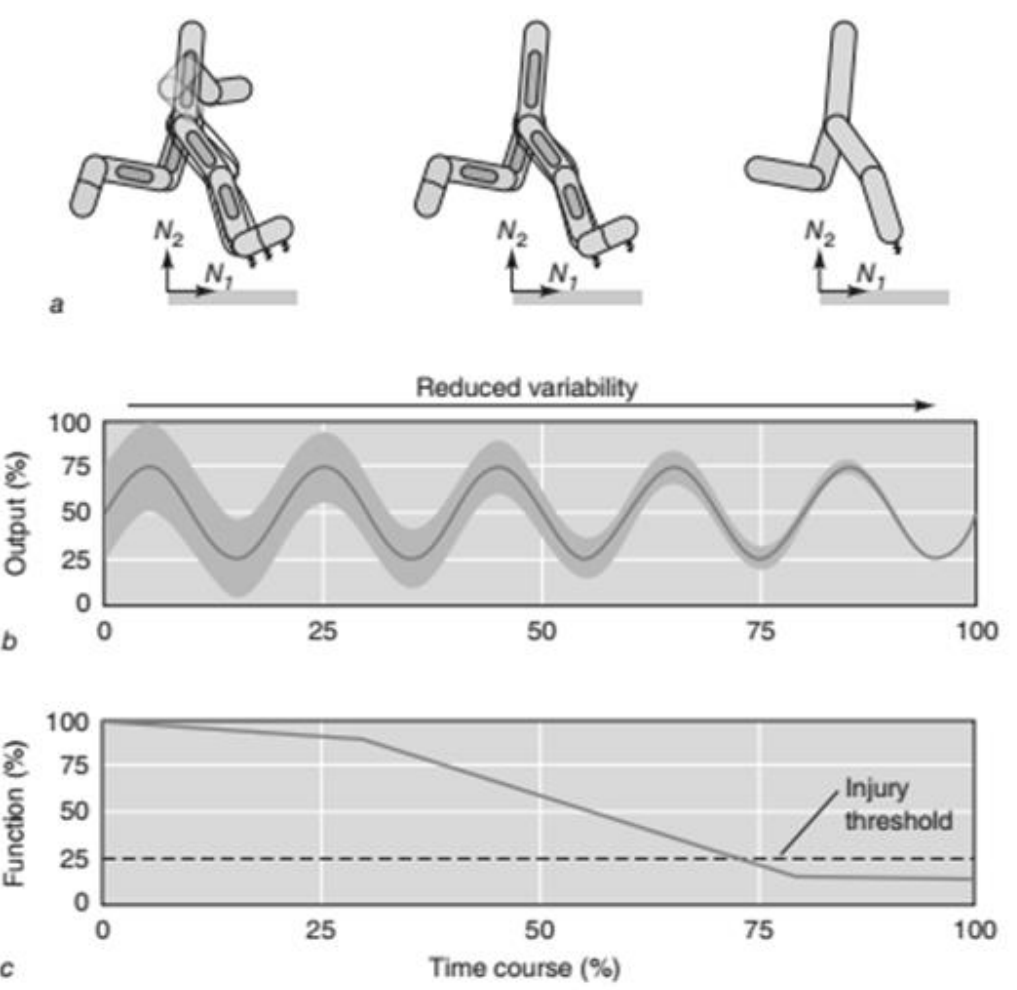

Fonte: (2002) adaptado de Robertson et al., (2013)

Legenda: Com o tempo, reduções nos graus de liberdade efetivos, componentes interativos e sinergias (a) tornam-se associados a uma perda de variabilidade no sistema (b). Quando estas reduções em graus de liberdade e variabilidade atingir um limiar crítico, lesões ou doenças podem emergir (c).

A hipótese de perda de complexidade de Lipsitz (2002) pode ser exemplificada em Van Emmerik; Van Wegen (2002). Estes autores observaram que jovens exibiam maior excursão do Centro de Pressão (COP) que idosos na tarefa de equilíbrio ortostático. Posteriormente, era requisitado aos grupos realizar uma pequena inclinação anterior de tronco e os resultados evidenciavam que os idosos aumentavam as oscilações posturais comparado aos jovens, ou seja, eles não conseguiam manter o controle postural. Baseado nessas premissas, é possível que a variabilidade ótima esteja 
situada entre os limites superior (muito instável) e inferior (estereotipado), e que esse intervalo indica a funcionalidade dela em relação as capacidades físicas do indivíduo, e suas demandas cotidianas. Contudo, a expansão destes conceitos não é restrita ao caso de lesão no aparelho locomotor, somente com a diminuição dos graus de liberdade, mas para quando esses graus de liberdade não são suficientemente dominados pelo executante no desempenho motor - exemplos de estágios de aprendizagem, como na comparação de atletas de alto rendimento e novatos (VAN EMMERIK; VAN WEGEN, 2002; STERGIOU; HARBOURNE; CAVANAUGH, 2006; GLAZIER et al., 2006; NEWELL; JAMES, 2008; STERGIOU; DECKER, 2011).

O domínio dos graus de liberdade disponíveis pode ser verificado em Vereijken; Whiting; Beek (1992) que analisaram participantes numa tarefa simulada em plataforma de esqui durante várias etapas de treinamento. Os participantes realizavam a tarefa de modo mais rígido, e que conforme aumentava as sessões, eles diminuíam a rigidez e utilizavam gestos fluidos com menor gasto energético.

Nesta mesma linha verificamos outro exemplo nas habilidades de precisão. Arutyunyan, Gurfinkel e Mirsky $(1968,1969)$ constataram que atiradores de elite possuíam menor da oscilação da mira ao alvo, durante o tiro, por meio da análise PILAT (Point of Interaction of Aiming and Target), em comparação a novatos. Contudo, os atiradores de elite realizavam maiores compensações no segmento ombropunho, o que impõe maior precisão na posição final do tiro, e os novatos não eram capazes de realizar tais ajustes, caracterizando o desempenho inferior pela maior oscilação da interação mira-e-alvo. Estas compensações ocorrem em outras modalidades. Stuart e Atha (1990) observaram que arqueiros de elite compensavam nas articulações de membros superiores e pescoço, considerando que não existe um padrão consistente que os arqueiros de elite adotam durante o tiro.

Além disto, ressalta-se a gama de estratégias de coordenação que atletas podem utilizar em suas atividades. Bauer; Schöllhorn (1998) analisaram dois arremessadores de disco em treinamento e competição, e constataram que as posições e as velocidades articulares foram diferentes. Tais atletas não produzem ações iguais e constantemente fazem ajustes necessários de acordo com as características ambientais que se situam. A análise dessas estratégias motoras, em qualquer movimento, é um dos produtos de como opera, no nível de controle, o complexo cérebro-corpo, de acordo com Guigon (2011). Nisto destacamos as abordagens dos Sistemas Dinâmicos (SD) e Hipótese de Ponto de Equilíbrio (HPE), cujas algumas das técnicas exploradas nelas serão utilizadas no presente trabalho. 


\subsubsection{Sistemas Dinâmicos (Dynamical Systems Approach)}

Nesta abordagem, o foco principal é sobre as transições de comportamentos motores e as ferramentas necessárias para adentrar seus problemas, com princípios que sugerem que, os padrões de movimento emergem em um estado organizacional sinérgico, baseado nos seguintes fatores: morfológicos (eg. anatomia topográfica); biomecânicos (eg. Leis de Newton); ambientais e os restritores da tarefa (eg. modulação da velocidade lenta e rápida na marcha). Esta abordagem sugere que a geração de padrões movimento é multifatorial envolvendo o acoplamento de múltiplos graus de liberdade, que será matematicamente modelado em equações diferenciais para descrever um sistema de fases comportamentais com essas complexidades expostas (STERGIOU, 2004; LATASH, 2008a).

A variabilidade de coordenação neste sistema é a quantidade potencial de padrões de coordenação que serão determinadas na ação humana. São as suas características principais: 1) a infinidade de possibililidades das taxas preferenciais ou periodicidades (atractores) no sistema. 2) a resistência destas taxas preferenciais a perturbações. 3) e caso as perturbações se tornam excessivas, o sistema pode se reorganizar (o que provoca a mudança ou deslocamento de fase), encontrando um novo patamar de estabilidade. (STERGIOU; DECKER, 2011). Latash (2008) exemplifica isto no modelo Halen-Kelso-Bunz (FIGURA 6): inicialmente a pessoa realiza o movimento coordenando em fase (com os dedos estendendo e fletindo na mesma frequência) ou fora de fase (um dedo estendendo outro fletindo) facilmente, após um período o padrão fora de fase muda para a coordenação em fase, ou seja um novo padrão emerge no sistema para estabilizá-lo, corroborando com esta abordagem no controle do movimento.

Figura 6. Coordenação bimanual

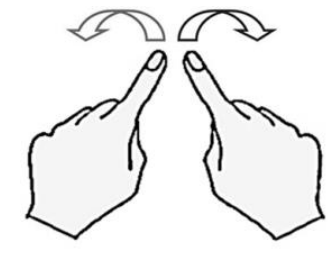

In-phase coordination

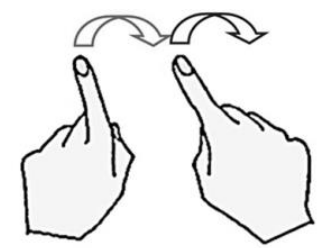

Anti-phase coordination

Fonte: Sleimen-Malkoun et al. (2014)

Legenda: A coordenação em fase consiste em movimentos simétricos em sentidos iguais $\left(0^{\circ}\right.$ de fase relativa) envolvendo a ativação simultânea de músculos homólogos. O padrão de coordenação antifase consiste em movimentos em sentidos opostos ( $180^{\circ}$ de fase relativa) envolvendo a ativação simultânea de músculos antagonistas (adaptado de). 
De modo resumido, a base das medidas de coordenação neste sistema está fundada nos atractores no campo espaço de estados. A Figura 7 representa um diagrama ângulo-ângulo, bilateral do segmento coxa na marcha humana. Nesta análise, é possível verificar o comportamento na transição de fases, e entre as medidas nos Sistemas Dinâmicos, destaca-se o Vector Coding (Análise de Vetor Codificado), cujas medidas podem ser empregadas na comparação de coordenação intersegmentar ou intermuscular em diferentes tipos de tarefa (VAN EMMERIK; MILLER; HAMILL, 2013).

Figura 7. Série temporal no diagrama ângulo-ângulo

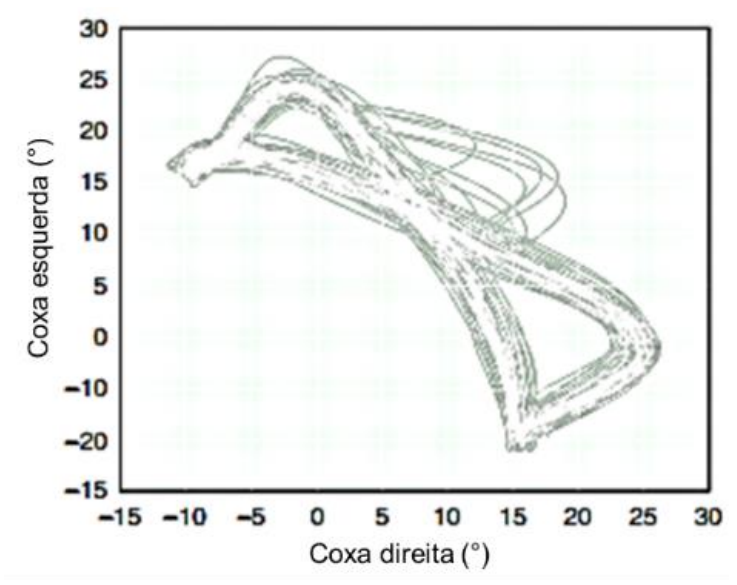

Fonte: Robertson et al. (2013).

Legenda: Acoplamento do segmento bilateral Coxa na marcha.

A Análise de Vetor Codificado tem origem em Freeman (1961), que concebeu uma técnica cadeia codificada quantificando a curva ângulo-ângulo. De acordo com Wheat; Glazier (2006), o procedimento usa a sobreposição em grades para transformar a trajetória ângulo-ângulo em elementos digitais, a união destes elementos em cadeia forma a direção dos segmentos, por meio de uma linha com intervalos de quadro-aquadro a cada dois pontos, o que leva combinação dos segmentos a formar curvas de um gráfico ângulo-ângulo (Figura 8a). Várias cadeias podem ser montadas, como a cadeia hexagonal (Figura 8b), onde a posição do segmento na cadeia estará em até 6 localizações possíveis. A evolução deste sistema hexagonal (Figura 8c) terá até 8 posições de acoplamento intersegmentar na coordenação do movimento (CHANG; VAN EMMERIK; HAMILL, 2008). 
Figura 8. Sequência evolutiva de Análise de Vetor Codificado

(a)

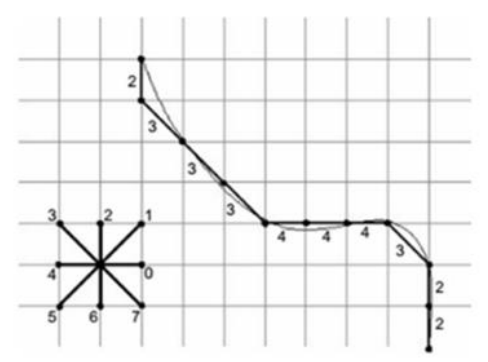

(b)

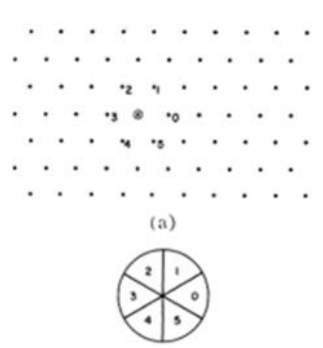

c)

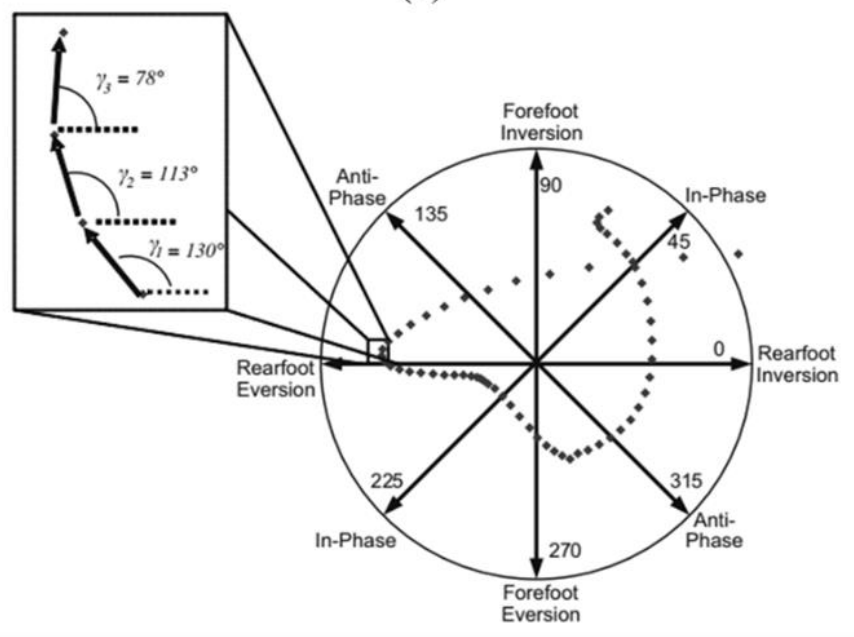

Fonte 8a: Freeman (1961) adaptado de Wheat; Glazier (2005);

Fonte 8b: Freeman (1961);

Fonte 8c: Chang; Van Emmerik; Hamill (2008).

Legenda: a) exemplo de encadeamento direcionado; b) cadeia codificada de posições hexagonais; c) Coordenação inter-segmentar entre retropé e antepé na marcha humana.

É importante salientar que esta técnica sofreu adaptações ao longo do tempo para melhor ajustá-la as particularidades e problemas de análise que eventualmente possam ocorrer, principalmente no deslocamento espacial equidistante ponto-a-ponto da análise e a trajetória analisada por pares, que não permite comparações múltiplas (SPARROW et al., 1987; TEPAVAC; FIELD-FOTE, 2001). A melhor solução foi flexibilizar estes pontos, sem normalização, garantindo os aspectos espaciais em detrimento dos temporais proposta por Hamill; Haddadm; Mcdermott (2000). O desenrolar desta técnica, no estudo da coordenação de segmentos e articulações, é uma das peças fundamentais para a compreensão do comportamento motor, desde as pessoas lesionadas até o alto rendimento de atletas (WHITING; ZERNICKE, 1982; MILLER et al., 2010; ALIBERTI, 2015; HEIDERSCHEIT; HAMILL; VAN EMMERIK, 2002).

Em grupos com Síndrome de Dor Patelofemoral, Heiderscheit; Hamill; Van Emmerik, (2002) identificaram, por meio desta técnica, que o acoplamento coxa-perna 
no plano transversal, era mais assimétrico em pessoas com esta síndrome comparado ao controle, e ainda havia uma menor variabilidade no membro acometido; num segundo momento, após a aplicação do tapping patelar, a variabilidade no membro acometido aumentou chegando próximo aos níveis encontrados no controle. Isso sugere níveis baixos de variabilidade podem ser um produto da dor na corrida. Com efeito, o corredor ajusta sua mecânica para evita-la, reduzindo assim a variabilidade no acoplamento.

O próximo passo importante na transição de fases, utilizando esta técnica, ocorreu no trabalho de análise da marcha de Chang; Van Emmerik; Hamill (2008). Acreditava-se que na fase de ataque do calcanhar ao solo até a fase final de apoio e propulsão, a estratégia de coordenação utilizada fosse prioritariamente de Antifase, entre o acoplamento dos segmentos retropé e antepé. Contudo, os autores encontraram diferentes resultados de frequência nos três planos de movimento (Figura 8c), no sagital: a moda de coordenação foi Em fase; coronal: a moda foi de Em fase seguido de Fase do antepé, e transversal; no início do apoio foi Fase de retropé, apoio-médio, Em Fase, e no terço final do movimento Fase do Antepé. Ou seja, estes vários parâmetros de coordenação encontrados evidenciam a gama de estratégias e a plasticidade na qual a pessoa as utiliza no simples ato da marcha.

A técnica possibilita identificar estratégias de coordenação tíbia-pé na corrida, onde existem diferentes coordenações de acordo com o sexo. Na fase de apoio, resposta à carga e propulsão, o padrão de coordenação é de eversão com rotação interna da tíbia na resposta à carga, e na propulsão espera-se a coordenação de inversão com rotação externa da tíbia. Rodrigues et al. (2015) evidenciaram que os corredores rotacionam externamente a tíbia enquanto o pé continua a everter no apoio médio, indicando uma estratégia proximal nesta transição. Em contraste, as corredoras iniciam a resposta à carga diretamente com inversão até a propulsão, e estes aspectos indicam que esta transição ocorre no sentido proximal-distal em homens e mais distal-proximal em mulheres. Isto pode ter relação com as mulheres realizam maior adução e rotação interna do quadril e tem maior fraqueza nos músculos desta articulação, limitando a habilidade de transição proximal-distal, invertendo o padrão de coordenação.

No salto triplo, os atletas com diferentes níveis de desempenho têm a variabilidade de coordenação dos acoplamentos de tornozelo-joelho e joelho-quadril em formato de curva em U, de acordo com o nível de habilidade. Enquanto atletas habilidosos e novatos possuem maior variabilidade, os atletas de nível intermediário possuem menor variabilidade, o que pode estar relacionado com a aprendizagem motora 
de atletas. Os atletas habilidosos possuem mais estratégias disponíveis e os de nível inferior as estão adquirindo para realizar a tarefa (WILSON et. al, 2008),

Todos estes exemplos evidenciam as possibilidadesde aplicação da análise de Vetor Codificado. O modelo esquemático de Chang; Van Emmerik; Hamill, (2008) (Figura 8c), adaptado em Van Emmerik; Miller; Hamill (2013) (Figura 9) pode ser empregado em qualquer acoplamento intersegmentar ou mesmo de coordenações entre músculos, como eletromiografia. Estas ações resultarão nas seguintes intepretações dos resultados: coordenação em fase: quando pares de músculos mudam de ativação no mesmo sentido; coordenação antifase: um músculo aumenta sua atividade e o seu par de análise diminui; coordenação Fase individual do músculo: quando um músculo ativa e o outro não muda a intensidade da atividade durante o movimento.

Figura 9. Classificação do padrão de coordenação de acordo com análise de Vetor Codificado

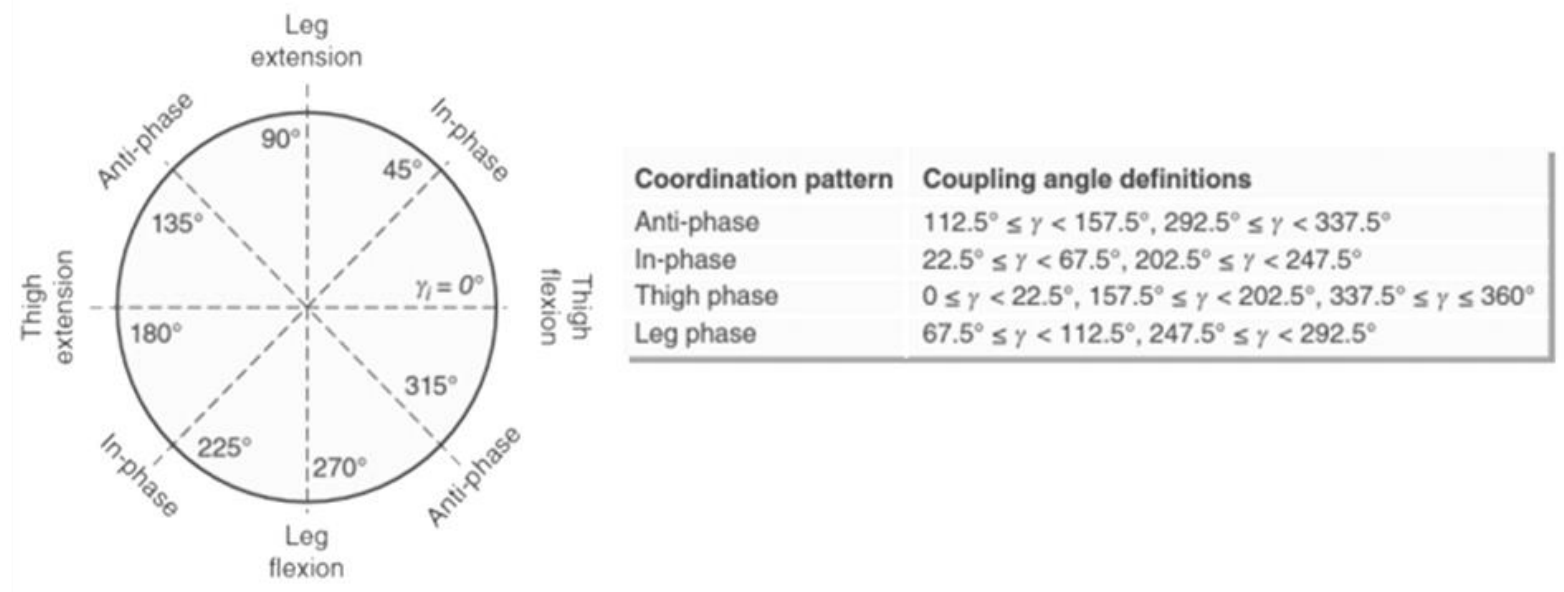

Fonte: Robertson et al. (2013)

A análise da variabilidade do movimento por meio da eletromiografia no estudo do desempenho humano é pouco usual, e apesar das limitações da ferramenta, alguns dados sugerem que de fato atletas variam as estratégias de coordenação muscular individual e entre sujeitos. Hug et al. (2008), na análise de ciclistas treinadas, pedalando em diferentes intensidades, verificaram diferentes respostas de atividade principalmente nos músculos biarticulares.

Certamente, o estudo do acoplamento de músculos com a análise de Vetor Codificado ainda é inédito na literatura, e ainda, existem questionamentos sobre as estratégias mais utilizadas por atletas de elite e a variabilidade ainda não foi estudada na modalidade, que é o caso do Tiro com Arco, onde encontramos diferentes estratégias de coordenação muscular. Existe variabilidade de níveis de atividade principalmente entre os músculos flexores superficiais dos dedos e extensores dos dedos. Pode ocorrer 
relaxamento e contração ativa extensora, geralmente evidente entre a queda do clicker e a completa liberação da flecha, o que favorece a utilização de ferramenta mais abrangente para responder as principais estratégias de coordenação que são utilizadas na modalidade (MARTIN; SILER; HOFFMAN, 1990; ERTAN et al., 2003; TINAZCI, 2011). O Clicker é o dispositivo acoplado ao arco do atleta, no qual a flecha é inserida entre ele e o arco. Serve para padronizar a puxada, que a coloca no local exato até onde ele precisa tracionar a corda para disparar. Quando liberado (clicker falls) provoca um estímulo audível e sensorial que, possibilita um melhor disparo da flecha (ERTAN et al., 2003).

As estratégias que podem ser utilizadas no movimento, evidentes no tipo de fase coordenativo do acoplamento muscular; o nível de co-ativação e inibição recíproca serão importantes para responder questões relativas a modulação da atividade do acoplamento ou par de músculos analisado, a partir da Hipótese do Ponto de Equilíbrio.

\subsubsection{Hipótese do Ponto de Equilíbrio}

A natureza desta abordagem sugere a presença de limiares (threshold) no sistema neuromuscular, na qual as posições de segmentos corporais são determinadas por meio do comprimento-tensão de grupos de músculos - se o músculo está abaixo deste limiar ele encontra-se desativado (silêncio), caso seja o contrário (acima do limiar) ele é ativado, e o nível desta ativação permanecerá no espaço entre o atual comprimento dele e o seu limiar - numa espécie de sistema tipo-mola (spring-like) (LATASH, 2008a; 2008b). Esta hipótese busca a resposta da pergunta do fisiologista Wachholder (como posso relaxar meus músculos em diferentes posições articulares?). Isso significa que, em um destes desdobramentos, um par de músculos agonista e antagonista pode relaxar em uma posição articular e em outras pode encurtar ou alongar (FIGURA 10) com comandos de co-ativação (C) e inibição reciproca (R) (LATASH, ZATSIORSKY, 2015). A co-ativação é a ativação simultânea de um par de músculos. O comando C leva a um aumento na amplitude articular, dentro da qual ambos os músculos agonistas e antagonistas são ativados sem uma mudança no ponto médio do intervalo (LATASH, 2008). A Inibição recíproca é diminuição da atividade antagonista e aumento da agonista (LATASH, 2008).

Em um par de músculos agonista-antagonista, o comportamento mecânico da articulação é o produto do momento/força total, com os pontos de estabilidade representados pela ação dos dois músculos (LATASH, ZATSIORSKY, 2015). Os comandos que emergiram deste comportamento são cruciais para coordenação do 
movimento. Os comandos de co-ativação e inibição recíproca limitam a amplitude de movimento, garantindo maior equilíbrio e estabilidade da articulação, são permissivos à liberdade de movimento da articulação para situações onde esta exigência torna-se necessária, e ambos os comandos são modulados por neurônios espinhais excitatórios e inibitórios (LATASH, 2008). A modulação destes comandos inibitórios e excitatórios influencia a magnitude dos ajustes posturais antecipatórios e compensatórios, que são realizados na ação humana refletindo nas estratégias adotadas pelas pessoas perante a perturbação. O Ajuste Postural Antecipatório (APA) é uma ação pré-programada, uma ativação de músculos posturais, que busca antecipar a magnitude da perturbação causada pelos segmentos corporais durante a preparação do movimento ou perturbação externa (MOCHIZUKI; AMADIO, 2003). O Ajuste Postural Compensatório (APC) é toda ação que ocorre após preparação do movimento ou perturbação externa, que pode ou não preceder o APA, no intuito de garantir a estabilidade do sistema (MOCHIZUKI; AMADIO, 2003). A modulação destes ajustes aumenta quando no uso de rifle, pois o recuo é uma perturbação que exige excelente controle postural, e o atirador habilidoso realizará ajustes antecipatórios que compensarão essa magnitude esperada do recuo (ARUTYUNYAN, GURFINKEL \& MIRSKY 1968, 1969; LATASH, 2008).

No Tiro com Arco, apesar dos trabalhos escassos, aparentemente estas modulações e ajustes também ocorrem em diferentes magnitudes nos atletas de alto rendimento; tanto nos aspectos de coordenação muscular como nos parâmetros de cinemáticos (ERTAN et al., 2003; STUART, ATHA 1990). Nesta modalidade, encontramos uma série de movimentos de preparação até o lançamento da flecha que possuem uma sequência estável, contudo o arqueiro aplica uma força constante que deforma o arco e acumula energia elástica. Quando o clicker é liberado iniciando o disparo do projétil. A energia é restituída, provocando além de uma perturbação postural, uma aceleração vertiginosa da flecha em direção ao alvo (STEFANYSHYN; WOROBETS, 2008). O tiro com arco é realizado a partir das seguintes etapas: 1) Segurar o arco, 2) pré-puxada da corda e 3) puxada final, subdividida em: 3.1) mirar, 3.2) disparar a flecha e 3.3) follow-through, quando o arqueiro acompanha a rotação em sentido horário do arco após o disparo (NISHIZONO et al., 1987). Neste momento (liberação do clicker), os ajustes posturais antecipatórios e compensatórios devem ocorrer com modulação dos comandos de inibição e co-ativação em função da perturbação. Na puxada final até o disparo, é observada a relação, por meio da análise eletromiográfica, entre nível de proficiência e capacidade de regular o tremor pelos 
músculos dorsais sugerindo que arqueiros habilidosos atiram com maior regularidade e fluidez (LEROYER; VAN HOECKE; HELAL, 1993).

Martin; Siler; Hoffman (1990) observaram que arqueiros de elite iniciavam um relaxamento dos músculos flexores superficiais dos dedos no lançamento da flecha. Contudo para os músculos extensores dos dedos, diferentes estratégias foram adotadas pelos arqueiros; oito deles realizavam o relaxamento total da musculatura extensora (Only Flexor's Strategy), enquanto sete arqueiros realizavam a contração ativa destes extensores imediatamente antes do lançamento da flecha (Active Extensor's Strategy). Deste modo, além das estratégias de coordenação, existem modulações e comandos antecipatórios que podem ser modificados ao longo da prática desta modalidade em atletas de alto desempenho, contudo tais dados ainda não foram medidos e evidenciados. Um dos meios efetivos para uma análise mais aprofundada é a análise dos índices de co-ativação e inibição reciproca anteriormente mostrados (LATASH, 2008).

Um dos meios de medir estes índices, com utilização do sinal eletromiográfico, são o de sobreposição, pela somatória da atividade muscular do par agonista-antagonista analisado para verificação do índice de co-ativação, e a supressão desta atividade muscular, pela subtração da atividade do par para o índice de inibição reciproca no período de interesse. Por exemplo, na postura em pé, os comandos típicos dos pares de músculos posturais são de inibição reciproca (R) (ARUIN, LATASH, 1995); contudo, na execução de tarefas rápidas de levantamento do braço, o comando altera-se para coativação (C), indicando que o sistema necessita de menor movimentação e maior estabilidade (SLIJPER; LATASH, 2000). O que torna esta ferramenta interessante para verificar a modulação destes comandos no Tiro com Arco, tendo em vista as diferentes estruturas coordenativas que os arqueiros utilizam para obter desempenhos de alta e baixa magnitude (TINAZCI, 2011). 


\section{MATERIAS E METÓDOS}

Este estudo observacional seguiu a diretriz do Conselho Nacional de Saúde, conforme aprovado pelo Comitê de Ética em Pesquisa da Escola de Artes, Ciências e Humanidades da Universidade de São Paulo, número: 1.452.196. Os voluntários foram esclarecidos sobre o experimento, local para a coleta e disponibilidade de horários. Foi lido o Termo de Consentimento Livre Esclarecido (TCLE) conforme resolução 196/96 do Conselho Nacional de Saúde para os participantes do estudo, e o voluntário assinou o termo de comprometimento ao estudo, participando do experimento.

Este projeto contou com um dia de coleta separado em duas etapas específicas, a primeira; foi marcada a localização para realização do procedimento no Clube Bonfim, e foi realizado o protocolo experimental com a aquisição do sinal eletromiográfíco.

\subsection{Amostra}

Onze arqueiros em nível de seleção brasileira em Tiro com Arco, incluindo cinco atletas que representaram o país nas Olímpiadas de 2016, participaram do presente estudo. Os critérios de elegibilidade adotados foram os de; não possuir lesão de membros superiores e tronco nos últimos seis meses, que possa interferir no estudo, e possuir pontuação FITA acima de 1200 pontos (a pontuação máxima da FITA é de 1440 pontos; normalmente escores entre 1300-1350 ganham competições internacionais de acordo com MARTIN; HEISE, 1992). Participaram cinco homens e seis mulheres $(25,2 \pm 4,9$ anos, $1,72 \pm 0,12 \mathrm{~m}, 68,6 \pm 11,8 \mathrm{~kg}$, FITA score $1258 \pm 43$ pontos, qualificatório em competições $632 \pm 20$ pontos, flecha média $8,7 \pm 0,3$ pontos). Todos os participantes assinaram o termo de consentimento, podendo desistir a qualquer momento da pesquisa (APÊNDICE I).

\subsection{Eletromiografia}

Para aquisição do sinal eletromiográfico, foi utilizado o eletromiográfo modelo 1610C (EMG Systems ${ }^{\circledR)}$ ). Foram utilizadas 16 entradas analógicas, separadas em 3 entradas para o acelerômetro (acoplado ao punho do lado dominante do arqueiro para sincronismo de todas fases da realização do Tiro com Arco), um para o trigger, para sincronizar eletromiografia e a liberação do clicker, e 12 entradas para o registro do sinal eletromiográfico.

Cada canal de entrada do sinal eletromiográfico possui um amplificador diferencial (Rejeição de Modo Comum >100dB) com ganho fixo 100. Os sinais foram devidamente amplificados, filtrados e digitalizados por um conversor A/D 16 bit com 
faixa de entrada de $\pm 2 \mathrm{~V}$. Dois eletrodos de superfície ativos foram posicionados no ventre muscular (ganho 20 vezes) de $\mathrm{Ag} / \mathrm{AgCl}$ circulares pré-amplificados com ganho 20 vezes. A distância intereletrodos foi $20 \mathrm{~mm}$.

Os dados eletromiográficos foram filtrados com filtro passa banda do tipo Butterworth de $4^{a}$ ordem com frequência de corte 10-450 Hz e filtro Notch para $60 \mathrm{~Hz}$ e harmônicas. Os dados foram filtrados e retificados com filtro passa baixa do tipo Butterworth de $4^{\mathrm{a}}$ ordem com frequência de corte $50 \mathrm{~Hz}$, depois foi utilizado o procedimento de Root Mean Square neste sinal e normalizado pelo valor $95 \%$ do máximo valor obtido. Para tratar o sinal destes dados foi utilizado o software Matlab 7.9.0 (R2009b). O sinal normalizado de cada músculo foi utilizado para duas análises distintas. Foram criados pares de músculos de índices de co-ativação e de inibição recíproca e expressos em módulo na unidade P.D.U. (Procedure Data Unit). Na segunda análise, o sinal de cada músculo foi utilizado para o desenvolvimento da análise de Vetor Codificado (VC), a partir do acoplamento de pares de músculos, na transição de fases dos ajustes, criando cinco transições (APA5-APA4; APA4-APA3; APA3-APA2; APA2-APA1; APA1-APC1).

\subsection{Acelerometria}

O acelerômetro foi acoplado no punho do lado dominante, compreendendo três entradas no sistema de aquisição de mesma marca (EMG Systems®), determinando o início do movimento, pico de velocidade e aceleração nos eixos ântero-posterior (x), látero-lateral (y), vertical (z). Os dados deste equipamento foram utilizados para sincronizar o sinal eletromiográfico com o início e fim do movimento do arqueiro.

\subsection{Posicionamento dos eletrodos}

Doze músculos foram avaliados: Reto Abdominal (RA), Multífido Lombar (ML), Flexor Superficial dos Dedos (FSD), Extensor dos dedos (ED), Bíceps Braquial (BB), Tríceps Braquial cabeça longa (TBL), Peitoral Maior (PM), Latíssimo do Dorso (LD) e Trapézio fibras Superiores (TS) foram avaliados no lado dominante; o Deltóide Posterior (DP) foi avaliado no lado não-dominante, e o Serrátil Anterior (SA) foi analisado em ambos os lados, o eletrodo referencial foi posicionado na clavícula.

Foi realizada a tricotomia e a abrasão da pele com algodão embebido em álcool $70 \%$ nos locais de fixação para remover a gordura na pele. Foi usada uma lixa água para remover as camadas sebáceas para aperfeiçoar a qualidade do sinal eletromiográfico. Os eletrodos foram fixados com fita adesiva. A recomendação para a fixação dos eletrodos 
sobre os músculos RA, ML, FSD, ED, BB, TBL, DP, TS, PM, SA e GD seguiu o Surface ElectroMyoGraphy for the Non-Invasive Assessment of Muscles (SENIAM) em acordo com Hermens et al. (2000); sobre o ventre do músculo, alinhado as fibras musculares. $\mathrm{O}$ posicionamento dos eletrodos para cada músculo foi: RA) $3 \mathrm{~cm}$ lateral a linha alba e $3 \mathrm{~cm}$ acima da cicatriz umbilical (CRAM; KASMAN; HOLTZ, 1998); ML) no nível de L2 e L3 deslocados lateralmente e alinhados com a porção caudal da espinha ilíaca póstero-superior (SENIAM, 2014); FSD e ED) foi requisitado ao arqueiro fazer flexão do punho e dos dedos, e em seguida foi palpado e colocado o eletrodo no ventre muscular do FSD; ED) o arqueiro realizou a extensão do punho e dos dedos e foi e colocado o eletrodo no ventre muscular (CRAM; KASMAN; HOLTZ, 1998); BB) 1/3 da distância entre o acrômio e a fossa cubital.; TBL) aproximadamente $2 \mathrm{~cm}$ mediais em relação ao braço com uma distância de $1 / 2$ entre a crista posterior do acrômio e o olecrano; DP) $2 \mathrm{~cm}$ abaixo da margem posterior do acrômio; GD) $4 \mathrm{~cm}$ abaixo do ângulo inferior da escápula; TS) aproximadamente 1/2 distância entre o acrômio e o processo espinhoso de C7 (SENIAM, 2014); PM ) aproximadamente 2cm da clavícula próximos a linha da axila; SA) abaixo da axila, ao nível do ângulo inferior da escápula e medial ao músculo GD (CRAM; KASMAN; HOLTZ, 1998).

\subsection{Protocolo}

O atleta realizou os tiros no ambiente outdoor e com distância de 70m (olímpica) para simular a etapa de qualificação e ranqueamento nas competições. $\mathrm{O}$ protocolo consistiu em aquecimento realizado em três blocos, cada bloco consistiu no lançamento de 6 flechas, totalizando 18 flechas de aquecimento. A seguir foi realizado o protocolo que teve os moldes do qualificatório utilizado nas competições nacionais e internacionais do Tiro com Arco. Este consistiu na realização de 12 blocos (6 tiros por bloco), ao final dos 6 primeiros blocos o arqueiro teve 20 minutos de intervalo, após o participante realizou os 6 blocos finais nos mesmos moldes aos anteriores. Foram coletadas a eletromiografia de todos os 72 lançamentos.

3.6 Variáveis de estudo

Variáveis dependentes: atividade eletromiográfica dos índices de inibição reciproca e co-contração durante o APA (0,5s antes da liberação do clicker) e APC (0,15s após a liberação do clicker $)$ que foram fragmentadas em distintos tempos:

Pré-APA: (500-250ms antes da queda do clicker) separados em APA 5 -500 400ms antes do clicker e APA 4 (350-250ms antes do clicker). 
APA: (250ms antes da queda do clicker até 50ms após a queda do clicker) separados em APA 3 (250-150ms antes do clicker); APA 2 (150-50ms antes do clicker); e APA 1 (50ms antes a 50ms após o clicker).

APC: Para o ajuste compensatório foi utilizado o APC1 no tempo de 50 a 150ms após o clicker.

Todos os ajustes foram analisados individualmente nos blocos, flechas e escores obtidos durante os tiros, na criação dos índices de Co-ativação e Inibição Recíproca e nas estratégias de coordenação dos pares músculos com análise de Vetor Codificado.

Variáveis independentes foram: a meta à 70m do arqueiro, os blocos setorizados em 12 e as seis flechas por blocos, de acordo com os moldes qualificatórios competitivos.

3.7 Construção dos Índices de Co-ativação e de Inibição Recíproca

O sinal eletromiográfico RMS normalizado foi utilizado para construção dos índices de Co-ativação (1) (C) e Inibição Reciproca (2) (R), com os pares agonistas e antagonistas de cada articulação, a partir dos seguintes procedimentos matemáticos (equações do estudo de Slipjer e Latash, 2000).

Índice $C=$ Agonista $[R M S[E M G$ norm $]]+$ Antagonista $[R M S[E M G n o r m]]$
Índice $R=\mid$ Agonista $[R M S[E M G$ norm $]]-$ Antagonista $[$ RMS $[$ EMGnorm $]] \mid$

Os pares analisados, agonista-antagonista (Tabela 1), foram construídos a partir da literatura acerca da ação muscular no tiro com arco (SOLYU et al. 2006; MARTIN \& SILLER, 1990; NISHIZONO et al. 1987; AXFORD, 2015).

Tabela 1. Pares de músculos agonistas-antagonistas analisados no presente estudo

\begin{tabular}{lll}
\hline Articulação & Agonista & Antagonista \\
\hline Punho & Flexor Superficial dos Dedos & Extensor dos Dedos \\
Cotovelo & Bíceps Braquial & Tríceps Braquial (cabeça longa) \\
Ombro & Grande dorsal & Peitoral Maior (cabeça clavicular) \\
Escápula & Trapézio Superior & Serrátil anterior \\
Tronco & Reto Abdominal & Multífido Lombar \\
\hline
\end{tabular}

Fonte: Fernando Carvalheiro Reiser (2019)

Usamos pares complementares, o primeiro relacionado ao conjunto de músculos do punho e do cotovelo, intitulado de par distal-proximal (Tabela 2). 
Tabela 2. Pares de músculos distal-proximal das articulações do punho e do cotovelo

\begin{tabular}{lll}
\hline Tipo & Distal & Proximal \\
\hline Agonistas & Flexor Superficial dos Dedos & Bíceps Braquial \\
Agonista-antagonista & Flexor Superficial dos Dedos & Tríceps Braquial (cabeça longa) \\
Antagonista-agonista & Extensor dos Dedos & Bíceps Braquial \\
Antagonistas & Extensor dos Dedos & Tríceps Braquial (cabeça longa) \\
\hline Fonte: Fernando Carvalheiro Reiser (2019) &
\end{tabular}

Noutro foram construídos pares mistos envolvendo os músculos analisados da articulação glenoumeral e da escápula, incluindo também os do lado contralateral - que segura o arco (Tabela 3).

Tabela 3. Pares de músculos criados entre a articulação glenoumeral e da escápula

\section{Índice Misto}

\begin{tabular}{lll}
\hline Agonistas (Glenoumeral-Escápula) & Grande Dorsal & Trapézio Superior \\
Antagonista (Glenoumeral-Escápula) & Peitoral Maior & Serrátil Anterior \\
Lado ND (lado que segura o arco) & Deltóide Posterior (ND) & Serrátil Anterior (ND) \\
Escápula ambos os lados & Serrátil Anterior & Serrátil Anterior (ND) \\
Extensores da glenoumeral & Grande Dorsal & Deltóide Posterior (ND)
\end{tabular}

Fonte: Fernando Carvalheiro Reiser (2019)

ND: membro superior não dominante

\subsection{Análise de Vetor Codificado}

A análise de Vetor Codificado contou com os mesmos pares de músculos utilizados na construção dos índices anteriores; o índice foi construído a partir do seguinte procedimento matemático (3); onde as coordenadas da ordenada e da abscissa são substituídas pelos músculos do par analisado, como o agonista e antagonista do movimento, por exemplo, e os períodos de análise são retirados da transição APA até APC; no presente estudo as transições solicitadas foram APA5-APA4; APA4-APA3; APA3-APA2; APA2-APA1; APA1-APC1. 


$$
\gamma_{j, i=\tan ^{-1}}\left(\frac{y_{j, i+1}-y_{j, i}}{x_{j, i+1}-x_{j, i}}\right)
$$

O resultado do acoplamento é dado a partir do arcotangente. As coordenadas na análise do acoplamento muscular permitem menos possibilidades que o trajeto articular original. Na equação 4 há um modelo esquemático do par FSD-ED para montagem do Vetor Codificado na transição do APA5 para o APA4, utilizando o RMS do sinal eletriomiográfico normalizado.

$$
\gamma_{j, i=\tan ^{-1}}\left(\frac{R M S_{[F S D a p a 4}-R M S_{[F S D a p a 5]}}{R M S_{[E D a p a 4]}-R M S_{[\text {EDapa }]}}\right)
$$

Os pares podem ter quatro tipos de coordenação (Figura 10); fase agonista, quando o músculo agonista muda a atividade enquanto a atividade do músculo antagonista é constante; em fase, ambos os músculos mudam a atividade da mesma forma, alterando a co-ativação; fase antagonista, o músculo antagonista muda a atividade e a atividade do agonista é constante; e antifase, quando os músculos mudam de atividade em sentidos opostos, alterando a inibição recíproca.

Figura 10. Sistema de coordenação para análise dos músculos

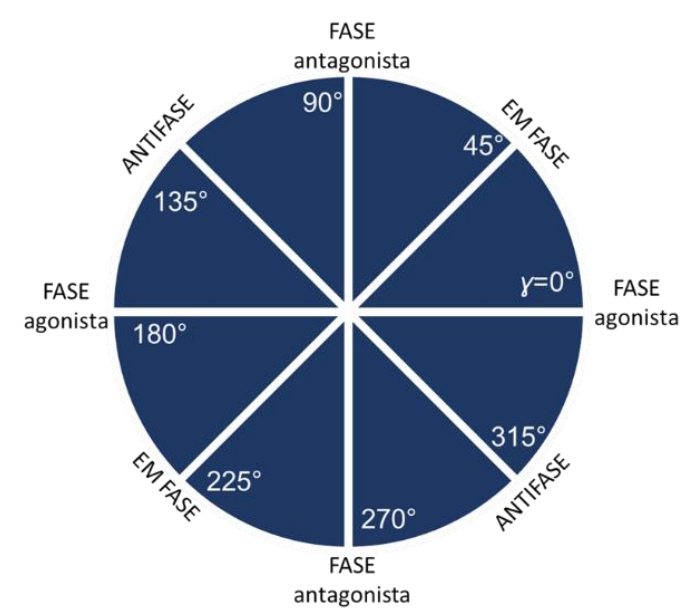

\begin{tabular}{|lc|}
\hline $\begin{array}{c}\text { TIPO DE } \\
\text { COORDENAÇÃO }\end{array}$ & \multicolumn{1}{c|}{ ÂNGULO DE ACOPLAMENTO } \\
\hline ANTIFASE & $112.5^{\circ} \leq \gamma<157.5^{\circ} ; 292.5^{\circ} \leq \gamma<337.5^{\circ}$ \\
\hline EM FASE & $22.5^{\circ} \leq \gamma<67.5^{\circ} ; 202.5^{\circ} \leq \gamma<247.5^{\circ}$ \\
\hline FASE AGONISTA & $0 \leq \gamma<22.5^{\circ} ; 157.5^{\circ} \leq \gamma<202.5^{\circ} ; 337.5^{\circ} \leq \gamma<360^{\circ}$ \\
\hline FASE ANTAGONISTA & $67.5^{\circ} \leq \gamma<112.5^{\circ} ; 247.5^{\circ} \leq \gamma<292.5^{\circ}$ \\
\hline
\end{tabular}

Fonte: Fernando Carvalheiro Reiser (2019)

O resultado das coordenadas, na transição de ajustes posturais, é obtido pela moda da coordenada; aquela que com mais frequência ocorre no par em questão. Este resultado será evidenciado no comportamento geral de todas as flechas disparadas e no desempenho obtido pelos arqueiros. 


\subsection{Análise estatística}

A análise estatística foi conduzida usando o software SPSS 23.0, expressados em médias e erro padrão, utilizando-se nos dados a significância estatística de $\mathrm{p}<0,05$, a análise de variância multivariada foi utilizada para verificar os pares que formaram os índices de Co-ativação e de Inibição Recíproca na análise dentro de cada APA e APC individualmente para bloco-a-bloco, flecha-a-flecha e escores. O teste Tukey HSD foi utilizado em casos de significância estatística nos cinco períodos dos ajustes posturais antecipatórios (APA) e para um período de ajuste postural compensatório (APC) individualmente. A técnica de Análise de Vetor Codificado foi utilizada estatisticamente com os procedimentos de frequência e moda de coordenação. 


\section{RESULTADOS}

4.1 Resultados descritivos de pontuação e eletromiografia

A análise da frequência na pontuação obtida pelas atletas está dividida na seguinte forma; dos 792 casos - escores 10 foram evidenciados: 147 vezes $(18,6 \%)$; escore 9: 264 vezes $(33,3 \%)$; escore 8: 206 vezes $(26,0 \%)$ e os escores $\leq 7: 175$ vezes $(22,1 \%)$. A análise descritiva do sinal eletromiográfico por ajuste postural está descrita na tabela 4 - dados estes utilizados para destacar também a atividade posteriormente agonista-antagonista nos pares de músculos analisados.

Tabela 4. Média e erro padrão do sinal eletromiográfico analisado

\begin{tabular}{|c|c|c|c|c|c|c|c|c|c|c|c|c|}
\hline & \multicolumn{2}{|c|}{ APA5 } & \multicolumn{2}{|c|}{ APA4 } & \multicolumn{2}{|c|}{ APA3 } & \multicolumn{2}{|c|}{ APA2 } & \multicolumn{2}{|c|}{ APA1 } & \multicolumn{2}{|c|}{ APC1 } \\
\hline & Média & EP & Média & EP & Média & EP & Média & EP & Média & EP & Média & EP \\
\hline FSD & 3,8 & 0,1 & 3,9 & 0,1 & 4,0 & 0,1 & 7,4 & 0,2 & 40,4 & 2,1 & 46,8 & 2,5 \\
\hline ED & 4,6 & 0,1 & 4,7 & 0,1 & 5,1 & 0,1 & 11,2 & 0,4 & 42,2 & 1,8 & 17,6 & 0,7 \\
\hline BBL & 5,1 & 0,1 & 5,1 & 0,1 & 5,2 & 0,1 & 10,1 & 0,3 & 23,2 & 0,9 & 13,4 & 0,5 \\
\hline TBL & 10,1 & 0,3 & 10,3 & 0,3 & 10,9 & 0,3 & 13,2 & 0,4 & 72,0 & 3,8 & 63,8 & 3,1 \\
\hline PMC & 7,8 & 0,4 & 6,3 & 0,3 & 6,7 & 0,3 & 16,6 & 0,7 & 42,5 & 1,9 & 43,8 & 2,1 \\
\hline RA & 5,1 & 0,3 & 6,1 & 0,3 & 8,1 & 0,4 & 8,8 & 0,5 & 17,8 & 1,0 & 28,7 & 1,6 \\
\hline SAD & 4,1 & 0,2 & 3,1 & 0,1 & 4,2 & 0,1 & 6,6 & 0,2 & 23,1 & 1,1 & 25,3 & 1,3 \\
\hline ML & 2,0 & 0,1 & 2,2 & 0,1 & 2,2 & 0,1 & 2,4 & 0,1 & 3,2 & 0,2 & 4,7 & 0,2 \\
\hline GD & 2,5 & 0,1 & 2,7 & 0,1 & 2,4 & 0,1 & 3,3 & 0,1 & 10,4 & 0,5 & 14,7 & 0,8 \\
\hline TS & 7,9 & 0,2 & 8,4 & 0,3 & 8,4 & 0,3 & 10,0 & 0,3 & 34,0 & 1,7 & 40,9 & 2,2 \\
\hline SAnD & 3,3 & 0,1 & 3,0 & 0,1 & 3,0 & 0,1 & 4,8 & 0,2 & 7,8 & 0,3 & 5,4 & 0,2 \\
\hline DPnD & 10,6 & 0,4 & 11,1 & 0,5 & 12,2 & 0,5 & 11,2 & 0,4 & 24,8 & 1,1 & 30,3 & 1,4 \\
\hline
\end{tabular}

\section{2 Índice de Co-ativação}

\subsubsection{Agonista-Antagonista por articulação}

Para os APA e APC, o par FSD-ED foi comparado entre blocos, flechas e escores (tabela 5). No APA5, o par FSD-ED não sofreu efeito do bloco, $\left(F_{11,520}=0.2\right.$, $\mathrm{p}=0.9)$; flecha $\left(F_{5,520}=0.5, \mathrm{p}=0.8\right)$ e escore $\left(F_{3,520}=0.9, \mathrm{p}=0.41\right)$. No APA4, esse par não sofreu efeito do bloco, $\left(F_{11,520}=0.5, \mathrm{p}=0.84\right)$; flecha $\left(F_{5,520}=0.4, \mathrm{p}=0.84\right)$ e escore $\left(F_{3,520}=1.8, \mathrm{p}=0.13\right)$. No APA3, esse par não mostrou efeito do bloco $\left(F_{11,520}=0.4\right.$, $\mathrm{p}=0.95)$; flechas $\left(F_{5,520}=0.3, \mathrm{p}=0.9\right)$ e escore $\left(F_{3,520}=2.4, \mathrm{p}=0.60\right)$. No APA2, esse par sofreu efeito do bloco $\left(F_{11,520}=2.3, \mathrm{p}<0.01\right)$; os testes revelaram maior atividade no bloco 3 em comparação com 1,7 e 10 (p<0,05). Não houve efeito da flecha $\left(F_{5,520}=0.8\right.$, $\mathrm{p}=0.5)$. Houve efeito de escore $\left(F_{3,520}=4.0, \mathrm{p}<0.01\right)$; e houve maior atividade escore 9 do que em 7 ( $\mathrm{p}<0.03)$. No APA1, não houve efeito do bloco $\left(F_{11,520}=1.8, \mathrm{p}=0.5\right)$, flechas $\left(F_{5,520}=0.8, \mathrm{p}=0.5\right)$ e escore $\left(F_{3,520}=1.3, \mathrm{p}=0.3\right)$. No APC1, não houve efeito do bloco $\left(F_{11,520}=1.7, \mathrm{p}=0.6\right)$ e das flechas $\left(F_{5,520}=0.3, \mathrm{p}=0.9\right)$, e houve efeito do escore 
$\left(F_{3,520}=4.0, \mathrm{p}<0.01\right)$; quando houve maior índice no escore 10 em comparação com 7 e 8 $(\mathrm{p}<0.01)$; e 9 e $7(\mathrm{p}<0.04)$.

Tabela 5. Co-ativação do par FSD-ED com Média e EP

\begin{tabular}{|c|c|c|c|c|c|c|c|c|c|c|c|c|}
\hline \multirow[t]{2}{*}{ Ends } & \multicolumn{2}{|c|}{$\begin{array}{c}\text { FSD-ED } \\
\text { (APA5) }\end{array}$} & \multicolumn{2}{|c|}{$\begin{array}{l}\text { FSD-ED } \\
\text { (APA4) }\end{array}$} & \multicolumn{2}{|c|}{$\begin{array}{c}\text { FSD-ED } \\
\text { (APA3) }\end{array}$} & \multicolumn{2}{|c|}{$\begin{array}{c}\text { FSD-ED } \\
\text { (APA2) }\end{array}$} & \multicolumn{2}{|c|}{$\begin{array}{c}\text { FSD-ED } \\
\text { (APA1) }\end{array}$} & \multicolumn{2}{|c|}{$\begin{array}{c}\text { FSD-ED } \\
\text { (APC1) }\end{array}$} \\
\hline & Mean & SE & Mean & $\mathrm{SE}$ & Mean & SE & Mean & SE & Mean & SE & Mean & SE \\
\hline 1 & 7,07 & 1,01 & 8,36 & 1,43 & 8,92 & 1,45 & 16,03 & $\underline{1,98}$ & 65,81 & 15,78 & 51,45 & 14,24 \\
\hline 2 & 9,48 & 1,46 & 9,88 & 1,50 & 9,88 & 1,40 & 22,11 & 2,93 & 105,10 & 16,77 & 79,51 & 17,56 \\
\hline 3 & 8,98 & 1,06 & 10,71 & 1,55 & 10,42 & 1,39 & 29,19 & $\underline{3,74}$ & $\underline{145,00}$ & 29,14 & 113,81 & 24,37 \\
\hline 4 & 8,24 & 1,13 & 9,33 & 1,39 & 9,09 & 1,16 & 17,56 & 2,00 & 107,75 & 20,98 & 55,12 & 13,17 \\
\hline 5 & 8,61 & 0,97 & 8,36 & 0,96 & 9,00 & 0,96 & 18,27 & 2,56 & 96,42 & 23,77 & 68,28 & 17,23 \\
\hline 6 & 9,00 & 0,97 & 8,82 & 0,95 & 10,28 & 1,22 & 18,78 & 2,35 & 81,98 & 15,53 & 65,18 & 17,42 \\
\hline 7 & 7,58 & 0,73 & 7,99 & 0,80 & 9,54 & 1,18 & $\underline{16,51}$ & $\underline{1,75}$ & 78,23 & 16,92 & 81,15 & 18,00 \\
\hline 8 & 7,96 & 0,83 & 7,88 & 0,77 & 8,68 & 0,88 & 19,17 & 6,55 & $\underline{53,07}$ & $\underline{13,82}$ & 53,30 & 14,57 \\
\hline 9 & 8,86 & 1,04 & 8,63 & 1,05 & 9,09 & 1,13 & 15,79 & 1,58 & $\underline{57,21}$ & $\underline{12,04}$ & $\underline{37,80}$ & $\underline{9,78}$ \\
\hline 10 & 8,86 & 0,95 & 7,55 & 0,72 & 8,06 & 0,74 & $\underline{12,64}$ & $\underline{1,24}$ & $\underline{54,23}$ & $\underline{11,16}$ & 53,24 & 12,34 \\
\hline 11 & 8,24 & 0,82 & 8,20 & 0,90 & 8,52 & 1,13 & 19,18 & 2,27 & 88,97 & 16,06 & 67,83 & 13,91 \\
\hline 12 & 8,14 & 1,05 & 7,93 & 0,77 & 8,07 & 0,73 & 17,14 & 2,38 & $\underline{57,08}$ & $\underline{10,32}$ & 45,24 & 11,48 \\
\hline \multicolumn{13}{|l|}{ Arrow } \\
\hline 1 & 7,36 & 0,56 & 7,74 & 0,76 & 8,06 & 0,75 & 17,72 & 3,43 & 66,38 & 10,72 & 56,89 & 10,56 \\
\hline 2 & 8,75 & 0,89 & 8,77 & 0,95 & 9,06 & 0,86 & 16,78 & 1,58 & 72,37 & 12,22 & 61,26 & 10,77 \\
\hline 3 & 8,30 & 0,68 & 8,81 & 0,82 & 9,53 & 0,86 & 19,13 & 1,82 & 86,83 & 13,69 & 54,35 & 9,80 \\
\hline 4 & 7,87 & 0,56 & 8,42 & 0,65 & 8,92 & 0,74 & 17,93 & 1,48 & 96,37 & 13,74 & 69,38 & 12,03 \\
\hline 5 & 9,48 & 0,85 & 9,40 & 0,82 & 9,44 & 0,75 & 20,66 & 1,94 & 89,78 & 13,97 & 70,39 & 11,43 \\
\hline 6 & 8,73 & 0,68 & 8,68 & 0,66 & 9,76 & 0,85 & 18,98 & 1,63 & 83,70 & 10,91 & 73,70 & 12,55 \\
\hline \multicolumn{13}{|l|}{ Score } \\
\hline 7 & 8,05 & 0,65 & 7,70 & 0,51 & 7,74 & 0,58 & $\underline{15,05}$ & $\underline{1,14}$ & 75,55 & 10,59 & $\underline{42,53}$ & $\underline{6,90}$ \\
\hline 8 & 8,41 & 0,66 & 8,92 & 0,71 & 9,02 & 0,66 & 17,92 & 2,34 & 69,09 & 8,53 & $\underline{56,99}$ & $\underline{8,60}$ \\
\hline 9 & 8,59 & 0,46 & 8,52 & 0,50 & 9,55 & 0,57 & $\underline{20,88}$ & $\underline{1,41}$ & 96,94 & 10,48 & $\underline{69,36}$ & $\underline{8,19}$ \\
\hline 10 & 8,54 & 0,59 & 9,57 & 0,87 & 10,19 & 0,86 & 19,32 & 1,41 & 84,01 & 10,51 & $\underline{91,51}$ & $\underline{13,10}$ \\
\hline
\end{tabular}

Fonte: Fernando Carvalheiro Reiser (2019)

Post hoc: $\mathrm{p}<0.05$ em itálico/sublinhado

Nos ajustes do cotovelo o índice BBL-TBL foi comparado entre blocos, flechas e escores (tabela 6). No APA5 o índice não sofreu efeito de bloco $\left(F_{11,520}=0.9 ; \mathrm{p}=0.52\right)$, flecha $\left(F_{5,520}=0.3 ; \mathrm{p}=0.87\right)$ e escore $\left(F_{3,520}=2.1 ; \mathrm{p}=0.1\right)$. No APA4 esse índice não sofreu efeito de bloco $\left(F_{11,520}=1.1 ; \mathrm{p}=0.32\right)$, flecha $\left(F_{5,520}=0.3 ; \mathrm{p}=0.9\right)$ e escore $\left(F_{3,520}=2.6\right.$; $\mathrm{p}=0.5)$. No APA3 esse índice sofreu efeito nos blocos $\left(F_{11,520}=2.0 ; \mathrm{p}=0.03\right)$, os testes revelaram maior atividade no bloco 4 em comparação ao 11 e ao 12 (p<0.05). Não houve efeito nas flechas $\left(F_{5,520}=1.1 ; \mathrm{p}=0.4\right)$. Nos escores houve efeito $\left(F_{3,520}=6.9\right.$; $\mathrm{p}<0.01$ ), e os testes revelaram maior atividade no escore 10 em comparação com o 7, 8 e 9 (p<0.03). No APA2 esse índice não sofreu efeito nos blocos $\left(F_{11,520}=1.0 ; \mathrm{p}=0.4\right)$, e flechas $\left(F_{5,520}=0.1 ; \mathrm{p}=0.99\right)$. Nos escores houve efeito $\left(F_{3,520}=8.8 ; \mathrm{p}<0.01\right)$, com maior atividade no escore 10 em comparação com 7, 8 e 9 ( $p<0.01)$. No APA1 esse índice não 
sofreu efeito dos blocos $\left(F_{11,520}=1.2 ; \mathrm{p}=0.26\right)$ e flechas $\left(F_{5,520}=0.6 ; \mathrm{p}=0.7\right)$. Houve efeito nos escores $\left(F_{3,520}=4.0 ; \mathrm{p}<0.01\right)$, maior no 10 em comparação ao $7(\mathrm{p}=0.05)$. Nesse índice o APC1 não teve efeito do bloco $\left(F_{11,520}=1.4 ; \mathrm{p}=0.2\right)$, e flechas $\left(F_{5,520}=0.4\right.$; $\mathrm{p}=0.9)$. Contudo houve efeito nos escores $\left(F_{3,520}=5.0 ; \mathrm{p}<0.01\right)$, maior no escore $10 \mathrm{em}$ comparação com 7 ( $\mathrm{p}<0.04)$.

Tabela 6. Co-ativação do par BBL-TBL com Média e EP

\begin{tabular}{|c|c|c|c|c|c|c|c|c|c|c|c|c|}
\hline \multirow[t]{2}{*}{ Ends } & \multicolumn{2}{|c|}{$\begin{array}{c}\text { BBL-TBL } \\
\text { (APA5) }\end{array}$} & \multicolumn{2}{|c|}{$\begin{array}{c}\text { BBL-TBL } \\
\text { (APA4) }\end{array}$} & \multicolumn{2}{|c|}{$\begin{array}{c}\text { BBL-TBL } \\
\text { (APA3) }\end{array}$} & \multicolumn{2}{|c|}{$\begin{array}{l}\text { BBL-TBL } \\
\text { (APA2) }\end{array}$} & \multicolumn{2}{|c|}{$\begin{array}{c}\text { BBL-TBL } \\
\text { (APA1) }\end{array}$} & \multicolumn{2}{|c|}{$\begin{array}{c}\text { BBL-TBL } \\
\text { (APC1) }\end{array}$} \\
\hline & Mean & SE & Mean & SE & Mean & SE & Mean & SE & Mean & SE & Mean & $\mathrm{SE}$ \\
\hline 1 & 11,18 & 1,41 & 11,94 & 1,45 & 12,9 & 1,7 & 20,8 & 2,8 & 59,4 & 10,7 & 42,6 & 8,2 \\
\hline 2 & 12,03 & 1,44 & 13,12 & 1,62 & 13,1 & 1,5 & 25,7 & 5,3 & 53,3 & 12,3 & 41,0 & 9,9 \\
\hline 3 & 15,85 & 2,21 & 16,58 & 2,39 & 16,8 & 2,4 & 22,1 & 3,3 & 58,5 & 18,8 & 82,5 & 19,4 \\
\hline 4 & 22,39 & 5,19 & 27,31 & 7,53 & $\underline{30,2}$ & $\underline{11,5}$ & 31,4 & 8,6 & 98,9 & 27,3 & 92,5 & 21,4 \\
\hline 5 & 16,60 & 2,60 & 17,99 & 3,53 & 20,0 & 4,3 & 23,7 & 3,3 & 98,1 & 27,3 & 79,2 & 19,8 \\
\hline 6 & 16,11 & 2,29 & 15,43 & 1,84 & 15,3 & 1,9 & 22,7 & 3,0 & 98,9 & 27,0 & 72,7 & 17,2 \\
\hline 7 & 21,57 & 8,40 & 16,74 & 3,00 & 18,6 & 4,7 & 26,7 & 3,9 & 113,9 & 25,5 & 82,3 & 16,9 \\
\hline 8 & 14,26 & 1,74 & 13,80 & 1,72 & 13,3 & 1,5 & 23,0 & 2,7 & 134,6 & 26,4 & 96,9 & 19,8 \\
\hline 9 & 12,51 & 1,64 & 14,31 & 1,94 & 14,8 & 1,9 & 22,5 & 3,0 & 105,0 & 23,1 & 71,8 & 15,4 \\
\hline 10 & 13,65 & 1,84 & 12,24 & 1,29 & 13,8 & 1,8 & 19,5 & 2,2 & 99,1 & 21,8 & 82,8 & 16,8 \\
\hline 11 & 12,42 & 1,64 & 11,88 & 1,29 & $\underline{12,2}$ & $\underline{1,4}$ & 21,9 & 2,7 & 117,1 & 21,9 & 96,5 & 17,6 \\
\hline 12 & 13,14 & 1,60 & 13,24 & 1,78 & $\underline{12,6}$ & $\underline{1,7}$ & 19,4 & 2,1 & 105,2 & 22,0 & 85,1 & 16,6 \\
\hline \multicolumn{13}{|l|}{ Arrow } \\
\hline 1 & 17,07 & 4,37 & 14,34 & 2,04 & 15,27 & 2,04 & 23,9 & 2,4 & 97,9 & 16,0 & 78,7 & 12,9 \\
\hline 2 & 13,60 & 1,85 & 13,71 & 1,28 & 17,29 & 5,48 & 22,5 & 4,4 & 72,8 & 13,6 & 73,3 & 11,1 \\
\hline 3 & 14,20 & 1,38 & 16,57 & 3,15 & 14,50 & 1,36 & 22,7 & 2,1 & 94,2 & 15,5 & 70,5 & 11,7 \\
\hline 4 & 13,68 & 1,20 & 14,23 & 1,41 & 17,17 & 2,70 & 22,1 & 2,0 & 96,2 & 17,4 & 68,6 & 11,4 \\
\hline 5 & 16,84 & 2,18 & 17,64 & 2,27 & 16,35 & 1,67 & 24,7 & 3,0 & 96,0 & 15,5 & 83,9 & 13,0 \\
\hline 6 & 15,46 & 1,67 & 15,80 & 1,98 & 16,24 & 2,14 & 23,9 & 2,1 & 113,8 & 18,0 & 87,9 & 12,2 \\
\hline \multicolumn{13}{|l|}{ Score } \\
\hline 7 & 12,16 & 1,62 & 11,97 & 1,61 & $\underline{11,00}$ & $\underline{1,08}$ & $\underline{16,6}$ & $\underline{1,6}$ & $\underline{67,9}$ & $\underline{12,0}$ & $\underline{53,3}$ & $\underline{9,3}$ \\
\hline 8 & 13,98 & 1,02 & 13,60 & 0,96 & $\underline{13,65}$ & $\underline{0,95}$ & $\underline{20,1}$ & $\underline{1,4}$ & 89,5 & 13,7 & 75,5 & 9,7 \\
\hline 9 & 15,06 & 1,16 & 16,93 & 2,00 & $\underline{16,35}$ & $\underline{1,53}$ & $\underline{24,1}$ & $\underline{1,6}$ & 104,8 & 11,3 & 81,8 & 8,6 \\
\hline 10 & 20,44 & 4,07 & 19,17 & 1,71 & $\underline{25,34}$ & $\underline{5,38}$ & $\underline{34,2}$ & $\underline{4,6}$ & 118,2 & $\underline{16,0}$ & $\underline{99,6}$ & $\underline{12,3}$ \\
\hline
\end{tabular}

Fonte: Fernando Carvalheiro Reiser (2019)

Post hoc: $\mathrm{p}<0.05$ em itálico/sublinhado

Nos ajustes do ombro o índice GD-PMC foi comparado entre blocos, flechas e escores (tabela 7). No APA5 o índice não sofreu efeito de bloco $\left(F_{11,520}=0.6 ; \mathrm{p}=0.9\right)$, flecha $\left(F_{5,520}=0.7 ; \mathrm{p}=0.63\right)$, e escore $\left(F_{3,520}<0.01 ; \mathrm{p}=0.99\right)$. No APA4 esse índice não sofreu efeito de bloco $\left(F_{11,520}=0.74 ; \mathrm{p}=0.7\right)$, flecha $\left(F_{5,520}=0.5 ; \mathrm{p}=0.74\right)$, e escore $\left(F_{3,520}=0.2 ; \mathrm{p}=0.85\right)$. No APA3 esse índice não sofreu efeito de bloco $\left(F_{11,520}=0.6\right.$; $\mathrm{p}=0.81)$, flechas $\left(F_{5,520}=0.1 ; \mathrm{p}=0.99\right)$, e escore $\left(F_{3,520}=0.2 ; \mathrm{p}=0.9\right)$. No APA2 esse índice não sofreu efeito de bloco $\left(F_{11,520}=0.6 ; \mathrm{p}=0.85\right)$, flecha $\left(F_{5,520}=0.2 ; \mathrm{p}=0.95\right)$, e escore 
$\left(F_{3,520}=1.7 ; \mathrm{p}=0.2\right)$. No APA1 esse índice não sofreu efeito de blocos $\left(F_{11,520}=0.6\right.$; $\mathrm{p}=0.86)$, flechas $\left(F_{5,520}=0.8 ; \mathrm{p}=0.6\right)$. Contudo, houve efeito de escore $\left(F_{3,520}=3.1\right.$; $\mathrm{p}=0.03)$, menor no 9 comparado ao 10 e 8 ( $\mathrm{p}<0.05)$. No APC1 não houve efeito do bloco $\left(F_{11,520}=0.7 ; \mathrm{p}=0.8\right)$, flechas $\left(F_{5,520}=0.8 ; \mathrm{p}=0.6\right)$, e escores $\left(F_{3,520}=0.8 ; \mathrm{p}=0.5\right)$.

Tabela 7. Co-ativação do par GD-PMC com Média e EP

\begin{tabular}{|c|c|c|c|c|c|c|c|c|c|c|c|c|}
\hline \multirow[t]{2}{*}{ Ends } & \multicolumn{2}{|c|}{$\begin{array}{l}\text { GD-PMC } \\
\text { (APA5) }\end{array}$} & \multicolumn{2}{|c|}{$\begin{array}{l}\text { GD-PMC } \\
\text { (APA4) }\end{array}$} & \multicolumn{2}{|c|}{$\begin{array}{l}\text { GD-PMC } \\
\text { (APA3) }\end{array}$} & \multicolumn{2}{|c|}{$\begin{array}{l}\text { GD-PMC } \\
\text { (APA2) }\end{array}$} & \multicolumn{2}{|c|}{$\begin{array}{l}\text { GD-PMC } \\
\text { (APA1) }\end{array}$} & \multicolumn{2}{|c|}{$\begin{array}{c}\text { GD-PMC } \\
\text { (APC1) }\end{array}$} \\
\hline & Mean & $\mathrm{SE}$ & Mean & SE & Mean & SE & Mean & SE & Mean & $\mathrm{SE}$ & Mean & SE \\
\hline 1 & 3,6 & 0,3 & 3,8 & 0,3 & 3,6 & 0,3 & 11,4 & 2,1 & 47,0 & 10,1 & 49,1 & 10,4 \\
\hline 2 & 16,2 & 6,3 & 22,9 & 8,1 & 19,2 & 6,1 & 33,4 & 9,8 & 54,6 & 13,1 & 52,9 & 11,9 \\
\hline 3 & 17,0 & 6,1 & 7,7 & 2,3 & 13,6 & 5,4 & 23,6 & 4,9 & 70,6 & 11,7 & 89,3 & 14,0 \\
\hline 4 & 7,1 & 3,2 & 5,2 & 1,5 & 8,3 & 3,9 & 16,6 & 4,0 & 50,5 & 10,0 & 67,4 & 12,6 \\
\hline 5 & 11,9 & 5,7 & 5,4 & 0,8 & 5,6 & 0,8 & 19,8 & 3,5 & 59,1 & 10,6 & 61,1 & 12,5 \\
\hline 6 & 16,7 & 7,3 & 15,7 & 7,7 & 10,8 & 4,5 & 18,7 & 4,4 & 55,1 & 11,5 & 60,1 & 12,8 \\
\hline 7 & 7,9 & 2,4 & 10,3 & 3,2 & 12,1 & 4,3 & 22,7 & 5,4 & 43,2 & 9,2 & 47,3 & 11,3 \\
\hline 8 & 5,6 & 1,9 & 7,2 & 2,5 & 6,2 & 1,7 & 15,2 & 3,1 & 39,8 & 8,0 & 35,5 & 8,3 \\
\hline 9 & 12,4 & 5,7 & 8,4 & 2,9 & 7,4 & 2,1 & 19,1 & 4,1 & 66,0 & 12,7 & 63,6 & 13,5 \\
\hline 10 & 6,7 & 2,9 & 4,0 & 0,3 & 4,4 & 0,4 & 16,1 & 3,2 & 50,3 & 9,2 & 54,8 & 10,0 \\
\hline 11 & 4,9 & 1,1 & 7,5 & 2,0 & 6,7 & 1,7 & 16,8 & 3,6 & 46,7 & 8,7 & 54,5 & 9,7 \\
\hline 12 & 13,2 & 4,9 & 9,5 & 3,7 & 11,7 & 4,9 & 24,7 & 6,1 & 52,0 & 11,8 & 66,9 & 12,1 \\
\hline \multicolumn{13}{|l|}{ Arrow } \\
\hline 1 & 7,6 & 2,4 & 6,2 & 1,6 & 7,4 & 2,3 & 18,6 & 3,4 & 53,7 & 7,5 & 54,1 & 8,0 \\
\hline 2 & 12,7 & 4,3 & 10,7 & 3,9 & 8,4 & 2,0 & 16,3 & 2,8 & 45,8 & 6,7 & 61,6 & 9,4 \\
\hline 3 & 8,4 & 2,7 & 8,2 & 2,1 & 8,2 & 2,3 & 20,8 & 3,3 & 60,6 & 8,5 & 63,3 & 8,4 \\
\hline 4 & 15,0 & 4,1 & 13,7 & 4,1 & 10,7 & 3,0 & 20,1 & 3,9 & 50,7 & 6,8 & 63,3 & 8,3 \\
\hline 5 & 10,2 & 3,0 & 8,2 & 1,8 & 9,1 & 2,4 & 20,4 & 3,8 & 48,3 & 7,8 & 50,7 & 7,9 \\
\hline 6 & 7,8 & 2,1 & 6,9 & 1,5 & 11,1 & 3,2 & 22,8 & 3,5 & 58,3 & 7,8 & 58,4 & 7,7 \\
\hline \multicolumn{13}{|l|}{ Score } \\
\hline 7 & 12,6 & 3,7 & 8,3 & 1,8 & 7,3 & 1,7 & 18,1 & 3,1 & 47,8 & 7,2 & 49,7 & 7,4 \\
\hline 8 & 10,1 & 2,7 & 10,6 & 3,3 & 8,4 & 1,9 & 21,1 & 3,0 & $\underline{60,9}$ & $\underline{6,5}$ & 63,7 & 7,1 \\
\hline 9 & 10,0 & 2,1 & 7,4 & 1,2 & 9,5 & 2,0 & 16,1 & 2,0 & $\underline{44,6}$ & $\underline{4,6}$ & 57,7 & 5,6 \\
\hline 10 & 8,4 & 1,8 & 10,4 & 2,3 & 11,8 & 2,8 & 27,0 & 3,7 & $\underline{62,5}$ & $\underline{6,9}$ & 63,3 & 7,4 \\
\hline
\end{tabular}

Fonte: Fernando Carvalheiro Reiser (2019)

Post hoc: $\mathrm{p}<0.05$ em itálico/sublinhado

Nos ajustes da escápula o índice TS-SAD foi comparado entre blocos, flechas e escores (tabela 8). Para o APA5, esse índice sofreu efeito do bloco, $\left(F_{11,520}=2.2\right.$; $\mathrm{p}=0.01$ ); contudo, o teste falhou na localização do efeito. Não houveram efeitos para flecha $\left(F_{5,520}=1.9 ; \mathrm{p}=0.1\right)$ e escore $\left(F_{3,520}=1.7 ; \mathrm{p}=0.2\right)$. No APA4, não houve efeito nos blocos $\left(F_{11,520}=1.42 ; \mathrm{p}=0.2\right)$, flecha $\left(F_{5,520}=1.1 ; \mathrm{p}=0.4\right)$ e escore $\left(F_{3,520}=0.6 ; \mathrm{p}=0.62\right)$. No APA3, não houve efeito nesse índice para bloco $\left(F_{11,520}=0.6 ; \mathrm{p}=0.9\right)$, flecha $\left(F_{5,520}=0.6\right.$; $\mathrm{p}=0.7)$ e escore $\left(F_{3,520}=0.34 ; \mathrm{p}=0.8\right)$. No APA2, não houve efeito de bloco $\left(F_{11,520}=1.9\right.$; $\mathrm{p}=0.23)$ e flecha $\left(F_{5,520}=1.8 ; \mathrm{p}=0.12\right)$. Houve efeito de escore $\left(F_{3,520}=2.5 ; \mathrm{p}=0.05\right) ; \mathrm{com}$ 
maior índice no escore 10 comparado ao 7 ( $\mathrm{p}<0.01)$. No APA1 houve efeito nos blocos $\left(F_{11,520}=2.5 ; \mathrm{p}<0.01\right)$; menor 9 em comparação ao 4 e $6(\mathrm{p}<0.05)$. Não houve efeito nas flechas $\left(F_{5,520}=0.1 ; \mathrm{p}=0.9\right)$. Houve efeito nos escores $\left(F_{3,520}=2.7 ; \mathrm{p}<0.05\right)$, com maior índice no 9 comparado ao 10 e $8(\mathrm{p}<0.05)$. Não houve efeito no APC1 do bloco $\left(F_{11,520}=1.4 ; \mathrm{p}=0.2\right)$, flechas $\left(F_{5,520}=0.9 ; \mathrm{p}=0.5\right)$, e escores $\left(F_{3,520}=0.9 ; \mathrm{p}=0.45\right)$.

Tabela 8. Co-ativação do par TP-SAD com Média e EP

\begin{tabular}{|c|c|c|c|c|c|c|c|c|c|c|c|c|}
\hline \multirow[t]{2}{*}{ Ends } & \multicolumn{2}{|c|}{$\begin{array}{l}\text { TS-SAD } \\
\text { (APA5) }\end{array}$} & \multicolumn{2}{|c|}{$\begin{array}{l}\text { TS-SAD } \\
\text { (APA4) }\end{array}$} & \multicolumn{2}{|c|}{$\begin{array}{l}\text { TS-SAD } \\
\text { (APA3) }\end{array}$} & \multicolumn{2}{|c|}{$\begin{array}{l}\text { TS-SAD } \\
\text { (APA2) }\end{array}$} & \multicolumn{2}{|c|}{$\begin{array}{l}\text { TS-SAD } \\
\text { (APA1) }\end{array}$} & \multicolumn{2}{|c|}{$\begin{array}{l}\text { TS-SAD } \\
\text { (APC1) }\end{array}$} \\
\hline & Mean & SE & Mean & SE & Mean & SE & Mean & SE & Mean & SE & Mean & SE \\
\hline 1 & 7,26 & 0,76 & 8,00 & 0,80 & 9,04 & 1,08 & 11,74 & 1,43 & 65,70 & 15,98 & 87,64 & 19,44 \\
\hline 2 & 8,26 & 0,91 & 7,25 & 0,80 & 9,40 & 1,26 & 16,54 & 5,19 & 40,27 & 9,02 & 67,61 & 17,49 \\
\hline 3 & 12,15 & 2,11 & 12,19 & 1,98 & 13,32 & 2,16 & 19,05 & 3,02 & 54,89 & 11,35 & 74,54 & 13,33 \\
\hline 4 & 13,02 & 2,29 & 10,44 & 1,76 & 9,67 & 1,56 & 22,65 & 5,88 & $\underline{89,21}$ & $\underline{17,31}$ & 100,10 & 22,68 \\
\hline 5 & 13,85 & 3,03 & 12,13 & 2,04 & 10,79 & 1,52 & 16,60 & 2,03 & 82,34 & 16,92 & 84,39 & 18,29 \\
\hline 6 & 16,67 & 4,33 & 12,37 & 1,95 & 12,74 & 2,16 & 17,84 & 2,21 & $\underline{88,43}$ & $\underline{16,95}$ & 79,95 & 15,43 \\
\hline 7 & 13,49 & 3,73 & 17,41 & 7,06 & 12,46 & 2,96 & 12,22 & 1,31 & 36,29 & 8,48 & 46,69 & 12,86 \\
\hline 8 & 11,05 & 1,67 & 9,55 & 1,19 & 10,22 & 1,53 & 15,17 & 2,32 & 42,39 & 7,13 & 45,19 & 9,70 \\
\hline 9 & 9,88 & 1,36 & 12,31 & 1,79 & 20,06 & 6,04 & 16,98 & 3,41 & 28,41 & 7,47 & 49,60 & 13,67 \\
\hline 10 & 10,06 & 1,43 & 10,15 & 1,42 & 11,16 & 1,50 & 15,08 & 2,14 & 46,87 & 9,07 & 44,84 & 10,59 \\
\hline 11 & 9,22 & 1,48 & 11,11 & 1,56 & 15,84 & 5,94 & 15,29 & 2,70 & 53,23 & 11,87 & 57,23 & 14,07 \\
\hline 12 & 18,71 & 5,36 & 14,64 & 3,19 & 16,52 & 6,85 & 20,95 & 4,62 & 56,70 & 9,67 & 56,61 & 13,09 \\
\hline \multicolumn{13}{|l|}{ Arrow } \\
\hline 1 & 9,82 & 1,31 & 9,79 & 0,84 & 13,28 & 3,02 & 15,57 & 1,91 & 58,87 & 9,02 & 69,14 & 12,81 \\
\hline 2 & 11,04 & 2,11 & 9,95 & 1,03 & 8,86 & 0,71 & 12,14 & 1,09 & 55,85 & 8,96 & 64,48 & 10,86 \\
\hline 3 & 10,85 & 1,18 & 9,74 & 1,01 & 10,30 & 1,01 & 14,97 & 1,26 & 58,31 & 8,54 & 71,18 & 10,99 \\
\hline 4 & 15,16 & 2,95 & 11,98 & 1,74 & 13,75 & 3,54 & 15,90 & 2,22 & 58,58 & 8,33 & 64,56 & 10,33 \\
\hline 5 & 13,38 & 2,20 & 14,98 & 3,66 & 16,25 & 3,39 & 21,34 & 3,45 & 62,10 & 10,69 & 53,98 & 10,12 \\
\hline 6 & 11,55 & 1,31 & 12,35 & 1,44 & 13,16 & 1,59 & 20,14 & 3,14 & 48,65 & 7,32 & 73,85 & 10,71 \\
\hline \multicolumn{13}{|l|}{ Score } \\
\hline 7 & 14,67 & 2,57 & 13,03 & 2,75 & 11,56 & 1,30 & $\underline{13,09}$ & $\underline{1,08}$ & 54,81 & 8,97 & 68,64 & 11,22 \\
\hline 8 & 11,44 & 1,12 & 11,98 & 1,28 & 13,60 & 2,37 & 15,81 & 1,74 & $\underline{47,60}$ & $\underline{5,93}$ & 54,44 & 7,89 \\
\hline 9 & 9,85 & 0,85 & 10,03 & 0,80 & 12,22 & 2,16 & 16,73 & 1,77 & $\underline{72,87}$ & $\underline{7,10}$ & 78,43 & 8,19 \\
\hline 10 & 13,30 & 2,04 & 11,45 & 1,10 & 13,12 & 1,25 & $\underline{22,06}$ & $\underline{2,99}$ & $\underline{44,61}$ & $\underline{5,40}$ & 57,81 & 8,08 \\
\hline
\end{tabular}

Fonte: Fernando Carvalheiro Reiser (2019)

Post hoc: $\mathrm{p}<0.05$ em itálico/sublinhado

Nos ajustes do tronco o índice RA-ML foi comparado entre blocos, flechas e escores (tabela 9), em média e erro padrão. No APA5, não houveram efeito nesse índice de bloco $\left(F_{11,520}=1.1 ; \mathrm{p}=0.4\right)$, flecha $\left(F_{5,520}=0.6 ; \mathrm{p}=0.73\right)$ e escore $\left(F_{3,520}=0.8 ; \mathrm{p}=0.6\right)$. No APA4, não houveram efeitos do bloco $\left(F_{11,520}=0.93\right.$; p $\left.=0.52\right)$, flecha $\left(F_{5,520}=0.3\right.$; $\mathrm{p}=0.9)$ e escore $\left(F_{3,520}=1.5 ; \mathrm{p}=0.21\right)$. No APA3, não houve efeito nesse índice do bloco $\left(F_{11,520}=0.9 ; \mathrm{p}=0.52\right)$, flecha $\left(F_{5,520}=0.3 ; \mathrm{p}=0.9\right)$ e escore $\left(F_{3,520}=1.0 ; \mathrm{p}=0.4\right)$. No APA2, não houve efeito nesse índice do bloco $\left(F_{11,520}=0.9 ; \mathrm{p}=0.6\right)$, flecha $\left(F_{5,520}=0.1 ; \mathrm{p}=0.99\right) \mathrm{e}$ escore $\left(F_{3,520}=1.6 ; \mathrm{p}=0.2\right)$. No APA1, não houve efeito nesse índice do bloco 
$\left(F_{11,520}=0.7 ; \mathrm{p}=0.71\right)$, flecha $\left(F_{5,520}=0.6 ; \mathrm{p}=0.7\right)$ e escore $\left(F_{3,520}=1.1 ; \mathrm{p}=0.34\right)$. No APC1 não houve efeito nesse índice do bloco $\left(F_{11,520}=1.5 ; \mathrm{p}=0.14\right)$, flecha $\left(F_{5,520}=1.2 ; \mathrm{p}=0.3\right) \mathrm{e}$ escores $\left(F_{3,520}=1.6 ; \mathrm{p}=0.2\right)$.

Tabela 9. Co-ativação do par RA-ML com Média e EP

\begin{tabular}{|c|c|c|c|c|c|c|c|c|c|c|c|c|}
\hline \multirow[t]{2}{*}{ Ends } & \multicolumn{2}{|c|}{$\begin{array}{l}\text { RA-ML } \\
\text { (APA5) }\end{array}$} & \multicolumn{2}{|c|}{$\begin{array}{l}\text { RA-ML } \\
\text { (APA4) }\end{array}$} & \multicolumn{2}{|c|}{$\begin{array}{l}\text { RA-ML } \\
\text { (APA3) }\end{array}$} & \multicolumn{2}{|c|}{$\begin{array}{l}\text { RA-ML } \\
\text { (APA2) }\end{array}$} & \multicolumn{2}{|c|}{$\begin{array}{l}\text { RA-ML } \\
\text { (APA1) }\end{array}$} & \multicolumn{2}{|c|}{$\begin{array}{l}\text { RA-ML } \\
\text { (APC1) }\end{array}$} \\
\hline & Mean & SE & Mean & SE & Mean & SE & Mean & SE & Mean & SE & Mean & SE \\
\hline 1 & 7,2 & 4,3 & 13,6 & 6,8 & 14,5 & 8,2 & 14,9 & 8,2 & 26,44 & 9,58 & 44,85 & 12,93 \\
\hline 2 & 2,4 & 0,5 & 6,4 & 0,8 & 2,4 & 0,4 & 3,3 & 0,5 & 24,28 & 6,74 & 49,59 & 15,56 \\
\hline 3 & 4,5 & 0,9 & 17,1 & 6,9 & 11,0 & 7,1 & 12,0 & 7,1 & 26,94 & 9,38 & 40,08 & 14,08 \\
\hline 4 & 4,5 & 1,1 & 10,1 & 1,7 & 12,0 & 4,1 & 7,9 & 2,1 & 15,04 & 5,93 & 22,93 & 8,99 \\
\hline 5 & 9,8 & 3,6 & 11,8 & 2,1 & 17,9 & 5,1 & 17,1 & 5,0 & 22,22 & 5,48 & 35,15 & 9,54 \\
\hline 6 & 5,4 & 1,5 & 11,4 & 2,0 & 4,8 & 1,3 & 7,5 & 1,9 & 11,18 & 3,06 & 16,14 & 4,24 \\
\hline 7 & 3,5 & 0,7 & 17,4 & 7,0 & 3,9 & 0,6 & 6,0 & 2,6 & 20,37 & 7,10 & 43,04 & 12,01 \\
\hline 8 & 12,2 & 5,7 & 17,7 & 5,6 & 12,2 & 7,0 & 8,3 & 3,8 & 21,95 & 8,93 & 19,05 & 6,49 \\
\hline 9 & 14,9 & 9,6 & 22,0 & 7,7 & 20,4 & 8,6 & 18,3 & 7,7 & 31,65 & 10,09 & 50,55 & 14,57 \\
\hline 10 & 4,0 & 1,5 & 13,4 & 3,8 & 6,1 & 2,2 & 9,8 & 5,0 & 22,56 & 7,59 & 37,30 & 11,95 \\
\hline 11 & 14,5 & 6,5 & 21,2 & 7,3 & 16,1 & 8,4 & 26,1 & 11,3 & 21,72 & 8,36 & 26,13 & 6,11 \\
\hline 12 & 2,2 & 0,4 & 11,9 & 2,9 & 1,8 & 0,2 & 3,0 & 0,5 & 7,88 & 2,78 & 16,45 & 6,10 \\
\hline \multicolumn{13}{|l|}{ Arrow } \\
\hline 1 & 5,2 & 2,2 & 13,8 & 3,9 & 10,6 & 4,4 & 10,59 & 4,45 & 20,84 & 5,28 & 37,06 & 8,42 \\
\hline 2 & 10,8 & 5,0 & 12,1 & 3,2 & 12,1 & 4,0 & 12,99 & 3,90 & 20,69 & 5,06 & 31,53 & 7,15 \\
\hline 3 & 5,8 & 1,8 & 14,7 & 4,2 & 11,3 & 4,2 & 11,02 & 4,00 & 26,10 & 6,38 & 29,43 & 6,56 \\
\hline 4 & 8,4 & 3,4 & 13,3 & 2,6 & 9,0 & 3,1 & 10,73 & 3,87 & 24,55 & 6,19 & 45,35 & 10,41 \\
\hline 5 & 4,8 & 1,4 & 15,0 & 3,9 & 5,0 & 1,3 & 7,85 & 3,02 & 16,55 & 3,88 & 28,67 & 6,75 \\
\hline 6 & 7,6 & 2,1 & 18,1 & 4,1 & 13,5 & 5,2 & 13,93 & 4,74 & 17,37 & 4,39 & 28,58 & 6,06 \\
\hline \multicolumn{13}{|l|}{ Score } \\
\hline 7 & 6,6 & 2,1 & 13,0 & 3,1 & 7,1 & 2,3 & 5,64 & 1,68 & 13,87 & 3,20 & 26,86 & 5,96 \\
\hline 8 & 5,8 & 1,6 & 15,5 & 2,9 & 8,7 & 3,1 & 9,35 & 2,76 & 23,06 & 4,42 & 28,18 & 5,50 \\
\hline 9 & 6,4 & 1,6 & 11,5 & 1,5 & 11,3 & 2,3 & 13,42 & 2,97 & 22,83 & 3,62 & 43,56 & 6,31 \\
\hline 10 & 10,7 & 4,6 & 20,2 & 5,2 & 14,3 & 5,4 & 16,32 & 5,53 & 23,41 & 6,25 & 30,47 & 6,89 \\
\hline
\end{tabular}

Fonte: Fernando Carvalheiro Reiser (2019)

4.2.2 Distal-Proximal (Punho e cotovelo)

O par agonista-agonista foi constituído pelos FSD-BBL (tabela 10) com média, erro padrão por bloco, flecha e escore. No APA5 não houve efeito nesse índice do bloco $\left(F_{11,520}=0.5 ; \mathrm{p}=0.9\right)$ e flecha $\left(F_{5,520}=0.2 ; \mathrm{p}=0.98\right)$. Houve efeito do escore $\left(F_{3,520}=3.1\right.$; $\mathrm{p}=0.03)$ maior no 10 comparado ao 7 ( $<<0.05)$. No APA4 não houve efeito nesse índice para bloco $\left(F_{11,520}=0.7 ; \mathrm{p}=0.8\right)$ e flecha $\left(F_{5,520}=0.5 ; \mathrm{p}=0.8\right)$. Houve efeito do escore $\left(F_{3,520}=4.2 ; \mathrm{p}<0.01\right)$; maior no escore 10 e 8 em comparação ao $7(\mathrm{p}<0.05)$. No APA3, houve efeito do bloco $\left(F_{11,520}=3.5 ; \mathrm{p}<0.01\right)$, contudo sem detecção no teste post hoc . Nenhum efeito para flechas $\left(F_{5,520}=2.0 ; \mathrm{p}=0.10\right)$. Houve efeito nos escores $\left(F_{3,520}=9.0\right.$; 
p <0.01) maior escore 10 em relação ao 7 ( $\mathrm{p}<0.01)$. Não houve efeito no APA2 do bloco $\left(F_{11,520}=1.5 ; \mathrm{p}=0.2\right)$, flecha $\left(F_{5,520}=0.3 ; \mathrm{p}=0.9\right)$ e escore $\left(F_{3,520}=2.1 ; \mathrm{p}=0.1\right)$ No APA1 não houve efeito do bloco $\left(F_{11,520}=1.2 ; \mathrm{p}=0.31\right)$, flecha $\left(F_{5,520}=0.43 ; \mathrm{p}=0.83\right)$ e escore $\left(F_{3,520}=0.7 ; \mathrm{p}=0.7\right)$. No APC1 houve efeito do bloco $\left(F_{11,520}=1.9 ; \mathrm{p}=0.04\right)$ contudo sem detecção pelo post hoc. Não houve efeito da flecha $\left(F_{5,520}=0.3 ; \mathrm{p}=0.93\right)$. Houve efeito do escores $\left(F_{3,520}=4.5 ; \mathrm{p}=0.04\right)$; maior 10 comparado $\operatorname{com} 7$ e $8(\mathrm{p}<0.01)$.

Tabela 10. Co-ativação do par FSD-BBL com Média e EP

\begin{tabular}{|c|c|c|c|c|c|c|c|c|c|c|c|c|}
\hline \multirow[t]{2}{*}{ Ends } & \multicolumn{2}{|c|}{$\begin{array}{l}\text { FSD-BBL } \\
\text { (APA5) }\end{array}$} & \multicolumn{2}{|c|}{$\begin{array}{l}\text { FSD-BBL } \\
\text { (APA4) }\end{array}$} & \multicolumn{2}{|c|}{$\begin{array}{l}\text { FSD-BBL } \\
\text { (APA3) }\end{array}$} & \multicolumn{2}{|c|}{$\begin{array}{c}\text { FSD-BBL } \\
\text { (APA2) }\end{array}$} & \multicolumn{2}{|c|}{$\begin{array}{l}\text { FSD-BBL } \\
\text { (APA1) }\end{array}$} & \multicolumn{2}{|c|}{$\begin{array}{l}\text { FSD-BBL } \\
\text { (APC1) }\end{array}$} \\
\hline & Mean & SE & Mean & SE & Mean & $\mathrm{SE}$ & Mean & SE & Mean & SE & Mean & SE \\
\hline 1 & 7,16 & 0,84 & 7,84 & 0,94 & 8,63 & 1,12 & 19,25 & 2,64 & 69,23 & 13,44 & 59,37 & 14,83 \\
\hline 2 & 9,13 & 1,26 & 10,19 & 1,44 & 10,28 & 1,41 & 22,89 & 3,40 & 67,96 & 13,56 & 69,85 & 17,02 \\
\hline 3 & 9,45 & 1,02 & 10,20 & 1,34 & 9,94 & 1,07 & 22,29 & 4,02 & 94,77 & 30,04 & 107,75 & 26,09 \\
\hline 4 & 8,35 & 0,91 & 9,15 & 1,21 & 7,97 & 0,79 & 18,10 & 2,44 & 83,74 & 20,57 & 54,75 & 14,02 \\
\hline 5 & 9,62 & 0,99 & 9,18 & 1,03 & 11,38 & 2,28 & 18,17 & 2,91 & 90,28 & 26,77 & 70,35 & 17,92 \\
\hline 6 & 9,92 & 1,13 & 9,99 & 1,12 & 10,20 & 1,22 & 17,05 & 2,29 & 55,49 & 15,24 & 45,96 & 12,66 \\
\hline 7 & 8,29 & 0,77 & 8,70 & 0,75 & 9,30 & 0,92 & 18,16 & 2,72 & 60,41 & 16,40 & 62,30 & 15,50 \\
\hline 8 & 8,81 & 1,05 & 9,40 & 1,14 & 9,19 & 1,00 & 14,03 & 1,92 & 37,66 & 9,84 & 46,17 & 12,91 \\
\hline 9 & 8,73 & 0,89 & 9,07 & 0,96 & 9,32 & 1,12 & 16,76 & 2,13 & 57,22 & 12,11 & 44,59 & 9,42 \\
\hline 10 & 9,37 & 0,95 & 8,16 & 0,74 & 8,66 & 0,80 & 13,30 & 1,50 & 45,81 & 12,00 & 55,73 & 12,76 \\
\hline 11 & 8,46 & 0,86 & 7,69 & 0,73 & 7,82 & 0,73 & 16,42 & 2,30 & 62,15 & 15,46 & 62,07 & 14,20 \\
\hline 12 & 8,96 & 0,89 & 8,87 & 0,90 & 8,70 & 0,86 & 13,34 & 1,66 & 37,76 & 7,52 & 43,06 & 11,20 \\
\hline \multicolumn{13}{|l|}{ Arrow } \\
\hline 1 & 8,01 & 0,59 & 7,74 & 0,61 & 8,23 & 0,59 & 16,41 & 1,67 & 54,37 & 9,72 & 55,32 & 10,49 \\
\hline 2 & 8,73 & 0,74 & 9,13 & 0,86 & 9,03 & 0,84 & 16,15 & 1,72 & 56,99 & 11,89 & 60,69 & 10,88 \\
\hline 3 & 8,91 & 0,66 & 8,90 & 0,69 & 9,11 & 0,73 & 18,40 & 2,06 & 69,92 & 13,97 & 53,46 & 9,84 \\
\hline 4 & 8,94 & 0,70 & 9,11 & 0,69 & 9,83 & 1,22 & 17,34 & 1,64 & 70,54 & 12,30 & 51,26 & 9,31 \\
\hline 5 & 9,53 & 0,77 & 10,20 & 0,85 & 9,87 & 0,75 & 19,85 & 2,23 & 73,37 & 14,73 & 69,04 & 11,82 \\
\hline 6 & 8,99 & 0,64 & 9,13 & 0,70 & 9,62 & 0,72 & 16,73 & 1,61 & 56,05 & 10,19 & 71,20 & 12,92 \\
\hline \multicolumn{13}{|l|}{ Score } \\
\hline 7 & $\underline{7,90}$ & $\underline{0,60}$ & $\underline{7,79}$ & $\underline{0,53}$ & $\underline{7,81}$ & $\underline{0,53}$ & 15,34 & 1,48 & 55,92 & 10,12 & $\underline{39,11}$ & $\underline{6,44}$ \\
\hline 8 & 9,13 & 0,60 & $\underline{9,55}$ & $\underline{0,67}$ & 9,40 & 0,66 & 17,03 & 1,33 & 59,75 & 8,53 & $\underline{51,25}$ & $\underline{7,76}$ \\
\hline 9 & 8,66 & 0,43 & 8,83 & 0,52 & 9,08 & 0,48 & 18,07 & 1,43 & 65,13 & 10,10 & 63,41 & 8,06 \\
\hline 10 & $\underline{9,94}$ & $\underline{0,66}$ & $\underline{10,17}$ & $\underline{0,69}$ & $\underline{11,23}$ & $\underline{1,15}$ & 19,60 & 1,76 & 75,07 & 10,48 & 91,86 & $\underline{13,50}$ \\
\hline
\end{tabular}

O agonista-antagonista, foi composto pelos músculos FSD-TBL (tabela 11), evidenciando médias e erro padrão para bloco, flechas e escores. Não houve efeito no APA5 do bloco $\left(F_{11,520}=1.1 ; \mathrm{p}=0.4\right)$, flecha $\left(F_{5,520}=0.5 ; \mathrm{p}=0.8\right)$ e escore $\left(F_{3,520}=1.6\right.$; $\mathrm{p}=0.1)$. Não houve efeito no APA4 do bloco $\left(F_{11,520}=1.3 ; \mathrm{p}=0.25\right)$, flecha $\left(F_{5,520}=0.3\right.$; $\mathrm{p}=0.9)$ e escore $\left(F_{3,520}=1.7 ; \mathrm{p}=0.2\right)$. Para esse índice no APA3 houve efeito do bloco $\left(F_{11,520}=1.9 ; \mathrm{p}=0.04\right)$, maior no 4 em relação $1,8,10-12$ ( $\left.\mathrm{p}<0.05\right)$, não houve efeito da flecha $\left(F_{5,520}=1.1 ; \mathrm{p}=0.35\right)$. Houve efeito do escore $\left(F_{3,520}=4.5 ; \mathrm{p}<0.01\right)$, maior $10 \mathrm{em}$ 
relação ao 8 e 7 (p<0.01). Neste índice para APA2 não houve efeito do bloco $\left(F_{11,520}=1.8 ; \mathrm{p}=0.1\right)$ e flechas $\left(F_{5,520}=0.5 ; \mathrm{p}=0.83\right)$. Houve efeito do escore $\left(F_{3,520}=4.4\right.$; p <0.01) com maior índice no 10 comparado ao 7 ( $<<0.01$ ). Não houve efeitos no APA1 do bloco $\left(F_{11,520}=0.7 ; \mathrm{p}=0.8\right)$, flecha $\left(F_{5,520}=0.32 ; \mathrm{p}=0.9\right)$ e escore $\left(F_{3,520}=1.9 ; \mathrm{p}=0.1\right)$.

Não houve efeito neste índice no APC1 do bloco $\left(F_{11,520}=1.6 ; \mathrm{p}=0.1\right)$ e flecha $\left(F_{5,520}=0.42 ; \mathrm{p}=0.84\right)$. Houve efeito no escore $\left(F_{3,520}=5.7 ; \mathrm{p}<0.01\right) ;$ maior $10 \mathrm{em}$ comparação com $7(\mathrm{p}<0.01)$.

Tabela 11. Co-ativação do par FSD-TBL com Média e EP

\begin{tabular}{|c|c|c|c|c|c|c|c|c|c|c|c|c|}
\hline \multirow[t]{2}{*}{ Ends } & \multicolumn{2}{|c|}{$\begin{array}{c}\text { FSD-TBL } \\
\text { (APA5) }\end{array}$} & \multicolumn{2}{|c|}{$\begin{array}{c}\text { FSD-TBL } \\
\text { (APA4) }\end{array}$} & \multicolumn{2}{|c|}{$\begin{array}{c}\text { FSD-TBL } \\
\text { (APA3) }\end{array}$} & \multicolumn{2}{|c|}{$\begin{array}{c}\text { FSD-TBL } \\
\text { (APA2) }\end{array}$} & \multicolumn{2}{|c|}{$\begin{array}{c}\text { FSD-TBL } \\
\text { (APA1) }\end{array}$} & \multicolumn{2}{|c|}{$\begin{array}{c}\text { FSD-TBL } \\
\text { (APC1) }\end{array}$} \\
\hline & Mean & SE & Mean & SE & Mean & SE & Mean & $\mathrm{SE}$ & Mean & SE & Mean & SE \\
\hline 1 & 9,76 & 0,98 & 10,23 & 0,98 & 11,11 & $\underline{1,15}$ & 17,25 & 1,86 & 71,01 & 16,29 & 60,66 & 13,68 \\
\hline 2 & 12,48 & 1,44 & 13,34 & 1,46 & 13,56 & 1,58 & 28,09 & 5,81 & 93,44 & 20,68 & 89,46 & 21,67 \\
\hline 3 & 14,28 & 1,92 & 15,09 & 1,97 & 14,80 & 1,95 & 21,60 & 3,98 & 117,38 & 39,60 & 162,29 & 37,46 \\
\hline 4 & 21,12 & 5,15 & 26,05 & 7,52 & 29,69 & $\underline{11,47}$ & 31,75 & 9,10 & 148,27 & 39,74 & 126,21 & 29,61 \\
\hline 5 & 14,70 & 2,49 & 16,29 & 3,48 & 16,37 & 3,72 & 19,42 & 2,91 & 137,73 & 44,11 & 125,87 & 31,39 \\
\hline 6 & 14,26 & 2,13 & 13,48 & 1,52 & 14,39 & 1,73 & 18,79 & 2,70 & 110,87 & 33,25 & 98,51 & 22,48 \\
\hline 7 & 20,76 & 8,38 & 16,19 & 2,97 & 17,93 & 4,62 & 21,29 & 3,30 & 114,48 & 30,63 & 114,38 & 24,02 \\
\hline 8 & 12,49 & 1,51 & 11,83 & 1,37 & 11,64 & $\underline{1,38}$ & 19,84 & 2,33 & 127,04 & 27,42 & 116,82 & 23,27 \\
\hline 9 & 11,25 & 1,48 & 13,60 & 1,87 & 14,01 & 1,77 & 17,68 & 2,32 & 91,95 & 24,57 & 75,31 & 17,13 \\
\hline 10 & 12,72 & 1,69 & 11,20 & 1,18 & 12,69 & $\underline{1,47}$ & 16,66 & 1,63 & 107,93 & 25,19 & 115,03 & 23,06 \\
\hline 11 & 11,46 & 1,43 & 11,65 & 1,20 & $\underline{11,35}$ & $\underline{1,27}$ & 17,96 & 2,20 & 128,41 & 28,85 & 137,00 & 26,17 \\
\hline 12 & 11,30 & 1,45 & 11,64 & 1,52 & $\underline{11,53}$ & $\underline{1,59}$ & 16,07 & 1,58 & 100,26 & 21,87 & 105,05 & 19,78 \\
\hline \multicolumn{13}{|l|}{ Arrow } \\
\hline 1 & 15,79 & 4,34 & 13,16 & 1,96 & 13,70 & 1,95 & 20,22 & 2,21 & 107,82 & 18,84 & 104,32 & 16,72 \\
\hline 2 & 13,34 & 1,86 & 12,77 & 1,18 & 17,30 & 5,48 & 21,71 & 4,58 & 90,97 & 19,08 & 102,80 & 16,07 \\
\hline 3 & 12,61 & 1,21 & 15,44 & 3,08 & 13,19 & 1,18 & 19,16 & 2,14 & 112,24 & 22,73 & 98,42 & 16,76 \\
\hline 4 & 11,79 & 0,94 & 12,99 & 1,31 & 15,21 & 2,44 & 19,37 & 1,86 & 115,41 & 23,21 & 94,54 & 16,33 \\
\hline 5 & 15,47 & 2,08 & 16,24 & 2,18 & 15,27 & 1,57 & 22,64 & 2,93 & 121,20 & 22,35 & 128,42 & 19,92 \\
\hline 6 & 14,29 & 1,62 & 14,70 & 1,89 & 14,87 & 2,05 & 20,10 & 1,99 & 126,76 & 22,38 & 134,79 & 19,65 \\
\hline \multicolumn{13}{|l|}{ Score } \\
\hline 7 & 11,50 & 1,56 & 11,58 & 1,56 & 10,39 & $\underline{1,02}$ & 15,17 & $\underline{1,43}$ & 82,97 & 16,04 & 71,59 & $\underline{11,90}$ \\
\hline 8 & 12,74 & 0,94 & 12,76 & 0,88 & $\underline{13,16}$ & $\underline{0,91}$ & 19,45 & 1,67 & 107,74 & 17,68 & 100,82 & 13,84 \\
\hline 9 & 13,78 & 1,10 & 15,41 & 1,95 & 15,07 & 1,47 & 21,11 & 1,50 & 127,48 & 16,07 & 121,41 & 12,85 \\
\hline 10 & 18,51 & 4,04 & 17,24 & 1,61 & 22,52 & $\underline{5,28}$ & 27,41 & $\underline{4,60}$ & 126,87 & 20,32 & $\underline{151,06}$ & 19,39 \\
\hline
\end{tabular}

O antagonista-agonista, foi composto pelos músculos EL-BBL em médias e erro padrão para bloco, flechas e escores (tabela 12). Não houve efeito no APA5 do bloco $\left(F_{11,520}=0.3 ; \mathrm{p}=0.9\right)$, flecha $\left(F_{5,520}=0.3 ; \mathrm{p}=0.93\right)$ e escore $\left(F_{3,520}=1.7 ; \mathrm{p}=0.2\right)$. Não houve efeito no APA4 do bloco $\left(F_{11,520}=0.53 ; \mathrm{p}=0.9\right)$, flecha $\left(F_{5,520}=0.2 ; \mathrm{p}=0.95\right)$. Houve efeito do escore $\left(F_{3,520}=2.9 ; \mathrm{p}=0.04\right)$ maior no 10 comparado ao $7(\mathrm{p}=0.05)$. Houve efeito no APA3 do bloco $\left(F_{11,520}=2.0 ; \mathrm{p}=0.03\right)$; mas o teste post hoc não detectou a diferença. 
Não houve efeito da flecha $\left(F_{5,520}=1.3 ; \mathrm{p}=0.3\right)$. Houve efeito do escore $\left(F_{3,520}=6.3\right.$; p<0.01) maior no 10 em relação ao 8 e 7 ( $\mathrm{p}<0.03)$. Não houve efeito no APA2 neste índice do bloco $\left(F_{11,520}=1.1 ; \mathrm{p}=0.4\right)$ e flecha $\left(F_{5,520}=1.3 ; \mathrm{p}=0.3\right)$. Houve efeito do escore $\left(F_{3,520}=5.0 ; \mathrm{p}<0.01\right)$ maior no 10 comparado a 7 e $8(\mathrm{p}<0.04)$, e 9 maior que $7(\mathrm{p}<0.01)$. Não houve efeito no APA1 do bloco $\left(F_{11,520}=1.1 ; \mathrm{p}=0.4\right)$ e flecha $\left(F_{5,520}=1.2 ; \mathrm{p}=0.3\right)$. Houve efeito do escore $\left(F_{3,520}=3.3 ; \mathrm{p}=0.02\right)$ maior no 9 comparado ao $8(\mathrm{p}=0.03)$. Não houve efeito no APC1 do bloco $\left(F_{11,520}=1.4 ; \mathrm{p}=0.2\right)$ e flecha $\left(F_{5,520}=1.0 ; \mathrm{p}=0.43\right)$, no escore o efeito ocorreu $\left(F_{3,520}=2.9 ; \mathrm{p}<0.04\right)$, contudo sem indicação no teste post hoc.

Tabela 12. Co-ativação do par ED-BBL com Média e EP

\begin{tabular}{|c|c|c|c|c|c|c|c|c|c|c|c|c|}
\hline \multirow[t]{2}{*}{ Ends } & \multicolumn{2}{|c|}{$\begin{array}{l}\text { ED-BBL } \\
\text { (APA5) }\end{array}$} & \multicolumn{2}{|c|}{$\begin{array}{l}\text { ED-BBL } \\
\text { (APA4) }\end{array}$} & \multicolumn{2}{|c|}{$\begin{array}{c}\text { ED-BBL } \\
\text { (APA3) }\end{array}$} & \multicolumn{2}{|c|}{$\begin{array}{c}\text { ED-BBL } \\
\text { (APA2) }\end{array}$} & \multicolumn{2}{|c|}{$\begin{array}{c}\text { ED-BBL } \\
\text { (APA1) }\end{array}$} & \multicolumn{2}{|c|}{$\begin{array}{l}\text { ED-BBL } \\
\text { (APC1) }\end{array}$} \\
\hline & Mean & SE & Mean & SE & Mean & SE & Mean & SE & Mean & SE & Mean & SE \\
\hline 1 & 8,48 & 1,45 & 10,07 & 1,93 & 10,76 & 2,03 & 19,54 & 2,96 & 54,20 & 10,28 & 33,42 & 7,86 \\
\hline 2 & 9,03 & 1,44 & 9,66 & 1,74 & 9,44 & 1,26 & 19,76 & 3,02 & 64,93 & 12,09 & 31,07 & 5,51 \\
\hline 3 & 10,54 & 1,39 & 12,19 & 2,06 & 12,44 & 1,85 & 29,73 & 3,70 & 86,14 & 12,01 & 33,99 & 4,38 \\
\hline 4 & 9,50 & 1,33 & 10,59 & 1,94 & 9,64 & 1,29 & 17,20 & 2,08 & 58,36 & 11,38 & 21,38 & 2,58 \\
\hline 5 & 10,51 & 1,32 & 10,07 & 1,31 & 12,64 & 2,45 & 22,57 & 3,41 & 56,76 & 8,96 & 21,58 & 2,53 \\
\hline 6 & 10,85 & 1,39 & 10,77 & 1,40 & 11,15 & 1,57 & 22,66 & 2,91 & 69,99 & 11,42 & 39,33 & 13,18 \\
\hline 7 & 8,39 & 0,91 & 8,53 & 0,92 & 10,21 & 1,44 & 21,87 & 3,00 & 77,70 & 14,62 & 49,11 & 13,01 \\
\hline 8 & 9,73 & 1,29 & 9,85 & 1,14 & 10,30 & 1,17 & 22,38 & 6,65 & 60,65 & 12,61 & 33,40 & 8,80 \\
\hline 9 & 10,11 & 1,22 & 9,35 & 1,19 & 9,91 & 1,41 & 20,56 & 2,68 & 70,26 & 12,12 & 34,26 & 7,85 \\
\hline 10 & 9,78 & 1,25 & 8,59 & 0,94 & 9,14 & 1,05 & 15,47 & 1,78 & 45,42 & 7,10 & 21,06 & 2,84 \\
\hline 11 & 9,19 & 1,18 & 8,43 & 1,02 & 9,38 & 1,30 & 23,08 & 2,94 & 77,61 & 12,25 & 27,38 & 4,89 \\
\hline 12 & 9,97 & 1,31 & 9,53 & 1,07 & 9,12 & 0,99 & 20,46 & 2,74 & 61,99 & 10,00 & 25,32 & 4,93 \\
\hline \multicolumn{13}{|l|}{ Arrow } \\
\hline 1 & 8,64 & 0,80 & 8,91 & 1,02 & 9,62 & 1,05 & 21,37 & 3,65 & 56,45 & 7,87 & 31,27 & 4,72 \\
\hline 2 & 9,01 & 0,93 & 9,70 & 1,15 & 9,05 & 0,84 & 17,52 & 1,76 & 54,24 & 7,16 & 31,71 & 5,72 \\
\hline 3 & 9,89 & 0,90 & 9,95 & 1,07 & 10,84 & 1,18 & 22,64 & 2,12 & 68,78 & 7,93 & 26,46 & 3,34 \\
\hline 4 & 9,76 & 0,90 & 9,66 & 0,87 & 10,89 & 1,37 & 20,62 & 1,83 & 77,20 & 9,38 & 43,47 & 8,82 \\
\hline 5 & 10,85 & 1,07 & 10,80 & 1,13 & 10,53 & 0,95 & 22,76 & 2,29 & 64,56 & 7,86 & 25,91 & 3,38 \\
\hline 6 & 9,90 & 0,86 & 9,79 & 0,83 & 11,13 & 1,06 & 22,73 & 2,12 & 70,78 & 7,94 & 26,82 & 3,21 \\
\hline \multicolumn{13}{|l|}{ Score } \\
\hline 7 & 8,72 & 0,80 & $\underline{8,09}$ & $\underline{0,58}$ & $\underline{8,35}$ & $\underline{0,62}$ & $\underline{16,49}$ & $\underline{1,42}$ & 60,49 & 6,63 & 24,19 & 2,82 \\
\hline 8 & 9,65 & 0,79 & 9,75 & 0,90 & $\underline{9,51}$ & $\underline{0,75}$ & $\underline{18,59}$ & $\underline{2,40}$ & $\underline{50,86}$ & $\underline{5,61}$ & 31,64 & 5,22 \\
\hline 9 & 9,88 & 0,60 & 10,04 & 0,68 & 10,83 & 0,74 & $\underline{23,83}$ & $\underline{1,63}$ & $\underline{74,24}$ & $\underline{6,29}$ & 29,77 & 3,32 \\
\hline 10 & 10,48 & 0,87 & $\underline{11,49}$ & $\underline{1,20}$ & 13,01 & $\underline{1,48}$ & $\underline{26,14}$ & $\underline{2,07}$ & 75,38 & 7,71 & 40,09 & 5,78 \\
\hline
\end{tabular}

$\mathrm{O}$ índice antagonista-antagonista, foi composto pelo EL-TBL em médias e erro padrão para bloco, flechas e escores (tabela 13). No APA5, não houve efeito do bloco $\left(F_{11,520}=0.9 ; \mathrm{p}=0.5\right)$, flecha $\left(F_{5,520}=0.4 ; \mathrm{p}=0.8\right)$, e escore $\left(F_{3,520}=1.5 ; \mathrm{p}=0.2\right)$. No APA4, não houve efeito do bloco $\left(F_{11,520}=1.3 ; \mathrm{p}=0.25\right)$, flecha $\left(F_{5,520}=0.3 ; \mathrm{p}=0.9\right)$ e escore 
$\left(F_{3,520}=2.0 ; \mathrm{p}=0.1\right)$. No APA3, neste índice houve efeito nos blocos $\left(F_{11,520}=1.9\right.$; $\mathrm{p}<0.04$ ); maior no 4 em comparação ao 1,2,8,10-12. Não houve efeito da flecha $\left(F_{5,520}=0.6 ; \mathrm{p}=0.6\right)$. Houve efeito do escore $\left(F_{3,520}=5.1 ; \mathrm{p}<0.01\right)$ maior no escore $10 \mathrm{em}$ comparação a 8 e $7(\mathrm{p}<0.01)$. No APA2, não houve efeito do bloco $\left(F_{11,520}=1.4 ; \mathrm{p}=0.2\right)$, e flechas $\left(F_{5,520}=0.4 ; \mathrm{p}=0.85\right)$. Houve efeito do escore $\left(F_{3,520}=7.9 ; \mathrm{p}<0.01\right)$ com maior índice no 10 comparado ao 8 e 7 ( $\mathrm{p}=0.03)$, e 9 maior que 7 ( $\mathrm{p}<0.01)$. No APA1, não houve efeito do bloco $\left(F_{11,520}=1.1 ; \mathrm{p}=0.4\right)$ e flecha $\left(F_{5,520}=1.5 ; \mathrm{p}=0.2\right)$. Houve efeito do escore $\left(F_{3,520}=4.5 ; \mathrm{p}<0.01\right)$ no 9 maior que $7(\mathrm{p}=0.02)$. Não houve efeito no APC1 do bloco $\left(F_{11,520}=1.5 ; \mathrm{p}=0.15\right)$ e flecha $\left(F_{5,520}=0.4 ; \mathrm{p}=0.8\right)$. Houveram efeitos do escore $\left(F_{3,520}=4.8 ; \mathrm{p}<0.01\right)$, maior no escore 10 comparado ao $7(\mathrm{p}=0.03)$.

Tabela 13. Co-ativação do par ED-TBL com Média e EP

\begin{tabular}{|c|c|c|c|c|c|c|c|c|c|c|c|c|}
\hline \multirow[t]{2}{*}{ Ends } & \multicolumn{2}{|c|}{$\begin{array}{l}\text { ED-TBL } \\
\text { (APA5) }\end{array}$} & \multicolumn{2}{|c|}{$\begin{array}{l}\text { ED-TBL } \\
\text { (APA4) }\end{array}$} & \multicolumn{2}{|c|}{$\begin{array}{l}\text { ED-TBL } \\
\text { (APA3) }\end{array}$} & \multicolumn{2}{|c|}{$\begin{array}{l}\text { ED-TBL } \\
\text { (APA2) }\end{array}$} & \multicolumn{2}{|c|}{$\begin{array}{l}\text { ED-TBL } \\
\text { (APA1) }\end{array}$} & \multicolumn{2}{|c|}{$\begin{array}{l}\text { ED-TBL } \\
\text { (APC1) }\end{array}$} \\
\hline & Mean & SE & Mean & SE & Mean & SE & Mean & SE & Mean & SE & Mean & SE \\
\hline 1 & 11,09 & 1,56 & 12,46 & 1,83 & 13,24 & 1,91 & 17,54 & 1,82 & 55,98 & 9,82 & 34,71 & 6,94 \\
\hline 2 & 12,38 & 1,58 & 12,82 & 1,63 & $\underline{12,72}$ & $\underline{1,36}$ & 24,96 & 4,89 & 90,42 & 15,26 & 50,68 & 10,31 \\
\hline 3 & 15,37 & 2,09 & 17,09 & 2,34 & 17,30 & 2,37 & 29,04 & 3,18 & 108,75 & 19,12 & 88,53 & 18,28 \\
\hline 4 & 22,27 & 5,17 & 27,49 & 7,53 & $\underline{31,37}$ & 11,44 & 30,85 & 8,33 & 122,89 & 26,38 & 92,84 & 20,52 \\
\hline 5 & 15,59 & 2,55 & 17,18 & 3,49 & 17,63 & 3,80 & 23,82 & 3,01 & 104,21 & 23,70 & 77,10 & 18,66 \\
\hline 6 & 15,18 & 2,12 & 14,26 & 1,60 & 15,35 & 1,84 & 24,39 & 2,88 & 125,37 & 24,44 & 91,89 & 21,29 \\
\hline 7 & 20,86 & 8,38 & 16,03 & 2,97 & 18,83 & 4,68 & 25,00 & 3,19 & 131,77 & 24,06 & 101,19 & 20,61 \\
\hline 8 & 13,41 & 1,48 & 12,28 & 1,31 & 12,75 & $\underline{1,39}$ & 28,19 & 6,66 & 150,03 & 25,43 & 104,05 & 20,51 \\
\hline 9 & 12,64 & 1,64 & 13,87 & 1,85 & 14,60 & 1,91 & 21,48 & 2,37 & 104,99 & 21,64 & 64,97 & 14,47 \\
\hline 10 & 13,14 & 1,79 & 11,63 & 1,20 & $\underline{13,16}$ & $\underline{1,63}$ & 18,83 & 1,83 & 107,54 & 20,21 & 80,35 & 16,33 \\
\hline 11 & 12,20 & 1,56 & 12,39 & 1,40 & 12,91 & $\underline{1,58}$ & 24,62 & 3,08 & 143,87 & 24,79 & 102,30 & 18,25 \\
\hline 12 & 12,32 & 1,67 & 12,30 & 1,55 & $\underline{11,95}$ & $\underline{1,47}$ & 23,18 & 3,31 & 124,49 & 24,03 & 87,31 & 16,98 \\
\hline \multicolumn{13}{|l|}{ Arrow } \\
\hline 1 & 16,42 & 4,36 & 14,33 & 2,08 & 15,10 & 2,08 & 25,17 & 3,79 & 109,90 & 15,42 & 80,28 & 12,47 \\
\hline 2 & 13,61 & 1,91 & 13,35 & 1,32 & 17,33 & 5,47 & 23,09 & 4,29 & 88,21 & 13,54 & 73,82 & 11,21 \\
\hline 3 & 13,59 & 1,30 & 16,49 & 3,14 & 14,92 & 1,39 & 23,40 & 1,88 & 111,09 & 14,81 & 71,42 & 11,55 \\
\hline 4 & 12,60 & 1,01 & 13,54 & 1,33 & 16,26 & 2,48 & 22,64 & 1,77 & 122,06 & 17,06 & 86,74 & 14,13 \\
\hline 5 & 16,79 & 2,17 & 16,84 & 2,20 & 15,92 & 1,60 & 25,56 & 2,91 & 112,39 & 14,96 & 85,29 & 12,90 \\
\hline 6 & 15,20 & 1,65 & 15,36 & 1,90 & 16,38 & 2,15 & 26,10 & 2,08 & 141,49 & 17,59 & 90,41 & 11,96 \\
\hline \multicolumn{13}{|l|}{ Score } \\
\hline 7 & 12,32 & 1,62 & 11,88 & 1,56 & $\underline{10,93}$ & $\underline{1,05}$ & $\underline{16,32}$ & $\underline{1,18}$ & $\underline{87,54}$ & $\underline{11,71}$ & $\underline{56,67}$ & $\underline{8,99}$ \\
\hline 8 & 13,26 & 1,02 & 12,96 & 0,98 & 13,27 & $\underline{0,89}$ & $\underline{21,00}$ & $\underline{2,38}$ & 98,85 & 12,84 & 81,20 & 10,63 \\
\hline 9 & 14,99 & 1,13 & 16,62 & 1,97 & 16,82 & 1,55 & $\underline{26,87}$ & $\underline{1,63}$ & $\underline{136,60}$ & $\underline{11,53}$ & 87,77 & 8,72 \\
\hline 10 & 19,04 & 4,05 & 18,57 & 1,76 & $\underline{24,30}$ & $\underline{5,31}$ & $\underline{33,95}$ & $\underline{4,37}$ & 127,18 & 15,01 & 99,29 & $\underline{12,47}$ \\
\hline
\end{tabular}

Fonte: Fernando Carvalheiro Reiser (2019)

Post hoc: $\mathrm{p}<0.05$ em itálico/sublinhado

4.2.3 Índice Glenoumeral-Escápula

Na tabela 14 está o índice PMC-SAD, com médias e erro padrão do bloco,

flechas e escores. Não houve efeito no APA5 para bloco $\left(F_{11,520}=1.3 ; \mathrm{p}=0.23\right)$, flecha 
$\left(F_{5,520}=2.0 ; \mathrm{p}=0.1\right)$ e escore $\left(F_{3,520}=0.4 ; \mathrm{p}=0.7\right)$. Não houve efeito no APA4 do bloco $\left(F_{11,520}=0.7 ; \mathrm{p}=0.8\right)$, flecha $\left(F_{5,520}=0.7 ; \mathrm{p}=0.7\right)$ e escore $\left(F_{3,520}=0.2 ; \mathrm{p}=0.9\right)$. Não houve efeito no APA3 do bloco $\left(F_{11,520}=0.5 ; \mathrm{p}=0.9\right)$, flecha $\left(F_{5,520}=0.2 ; \mathrm{p}=0.9\right)$ e escore $\left(F_{3,520}=0.4 ; \mathrm{p}=0.7\right)$. Não houve efeito nesse índice no APA2 do bloco $\left(F_{11,520}=0.6\right.$; $\mathrm{p}=0.9)$ e flecha $\left(F_{5,520}=0.5 ; \mathrm{p}=0.8\right)$. Houve efeito do escore $\left(F_{3,520}=3.0 ; \mathrm{p}=0.04\right)$, maior o 10 comparado 9 e 7 ( $\mathrm{p}<0.02)$. Não houve efeito no APA1 do bloco $\left(F_{11,520}=0.7 ; \mathrm{p}=0.7\right)$ e flecha $\left(F_{5,520}=0.7 ; \mathrm{p}=0.6\right)$. Houve efeito do escore $\left(F_{3,520}=3.2 ; \mathrm{p}=0.03\right)$, maior no $10 \mathrm{e}$ 9 comparados ao 7 ( $\mathrm{p}<0.05)$. Não houve efeito no APC1 do bloco $\left(F_{11,520}=0.5 ; \mathrm{p}=0.9\right)$ e flecha $\left(F_{5,520}=1.3 ; \mathrm{p}=0.2\right)$. Houve efeito do escore $\left(F_{3,520}=3.5 ; \mathrm{p}=0.01\right)$ maior no 10 e 9 comparado ao $7(\mathrm{p}<0.02)$.

Tabela 14. Co-ativação do par PMC-SAD com Média e EP

\begin{tabular}{|c|c|c|c|c|c|c|c|c|c|c|c|c|}
\hline \multirow[t]{2}{*}{ Ends } & \multicolumn{2}{|c|}{$\begin{array}{c}\text { PMC-SAD } \\
\text { (APA5) }\end{array}$} & \multicolumn{2}{|c|}{$\begin{array}{c}\text { PMC-SAD } \\
\text { (APA4) }\end{array}$} & \multicolumn{2}{|c|}{$\begin{array}{c}\text { PMC-SAD } \\
\text { (APA3) }\end{array}$} & \multicolumn{2}{|c|}{$\begin{array}{c}\text { PMC-SAD } \\
\text { (APA2) }\end{array}$} & \multicolumn{2}{|c|}{$\begin{array}{c}\text { PMC-SAD } \\
\text { (APA1) }\end{array}$} & \multicolumn{2}{|c|}{$\begin{array}{c}\text { PMC-SAD } \\
\text { (APC1) }\end{array}$} \\
\hline & Mean & SE & Mean & SE & Mean & SE & Mean & SE & Mean & SE & Mean & SE \\
\hline 1 & 3,57 & 0,38 & 4,12 & 0,50 & 4,40 & 0,50 & 12,73 & 2,23 & 22,88 & 5,28 & 62,14 & 16,25 \\
\hline 2 & 16,74 & 6,31 & 22,69 & 8,05 & 20,34 & 6,06 & 36,76 & 9,90 & 20,91 & 4,10 & 60,51 & 14,20 \\
\hline 3 & 18,11 & 6,14 & 8,61 & 2,44 & 15,65 & 5,46 & 30,13 & 5,51 & 33,68 & 8,15 & 80,79 & 15,73 \\
\hline 4 & 8,36 & 3,27 & 5,19 & 1,64 & 8,47 & 3,93 & 18,61 & 4,16 & 32,63 & 7,82 & 79,03 & 15,62 \\
\hline 5 & 14,85 & 6,94 & 5,35 & 0,78 & 5,80 & 0,89 & 23,50 & 3,97 & 44,00 & 8,27 & 74,81 & 15,11 \\
\hline 6 & 20,99 & 8,27 & 15,67 & 7,65 & 11,23 & 4,51 & 22,72 & 4,65 & 38,79 & 8,56 & 74,74 & 16,09 \\
\hline 7 & 6,34 & 1,76 & 8,60 & 2,15 & 11,98 & 4,29 & 20,79 & 4,88 & 25,12 & 6,16 & 51,67 & 13,42 \\
\hline 8 & 6,09 & 1,89 & 8,62 & 2,84 & 7,09 & 2,00 & 20,27 & 3,47 & 30,88 & 5,60 & 63,99 & 13,64 \\
\hline 9 & 13,27 & 5,71 & 9,69 & 2,88 & 15,56 & 6,19 & 24,06 & 5,03 & 16,69 & 3,13 & 70,18 & 14,79 \\
\hline 10 & 6,74 & 2,88 & 4,30 & 0,48 & 4,81 & 0,58 & 20,28 & 3,88 & 38,41 & 8,23 & 67,96 & 14,91 \\
\hline 11 & 5,29 & 1,08 & 8,60 & 2,05 & 13,24 & 6,06 & 19,93 & 4,14 & 33,14 & 8,57 & 62,16 & 15,46 \\
\hline 12 & 22,01 & 8,13 & 11,59 & 3,87 & 12,16 & 4,91 & 29,13 & 6,00 & 33,59 & 7,43 & 81,33 & 14,81 \\
\hline \multicolumn{13}{|l|}{ Arrow } \\
\hline 1 & 9,22 & 2,65 & 7,06 & 1,61 & 11,58 & 3,70 & 23,19 & 3,71 & 26,58 & 4,41 & 57,51 & 8,61 \\
\hline 2 & 15,02 & 4,75 & 11,55 & 3,92 & 9,18 & 2,03 & 18,19 & 2,76 & 28,58 & 5,19 & 67,98 & 11,87 \\
\hline 3 & 8,25 & 2,57 & 7,56 & 1,77 & 8,23 & 2,28 & 22,22 & 3,20 & 34,82 & 4,94 & 82,45 & 11,58 \\
\hline 4 & 20,49 & 5,51 & 14,21 & 4,10 & 11,15 & 2,98 & 22,60 & 3,98 & 30,56 & 5,09 & 69,99 & 10,60 \\
\hline 5 & 9,88 & 3,03 & 8,43 & 1,91 & 13,15 & 3,81 & 26,09 & 4,18 & 33,81 & 5,64 & 55,58 & 8,70 \\
\hline 6 & 8,30 & 2,11 & 7,71 & 1,51 & 12,07 & 3,21 & 27,17 & 3,88 & 31,01 & 4,48 & 81,14 & 11,55 \\
\hline \multicolumn{13}{|l|}{ Score } \\
\hline 7 & 15,75 & 4,17 & 9,30 & 1,81 & 8,20 & 1,72 & $\underline{18,80}$ & $\underline{3,11}$ & $\underline{21,52}$ & $\underline{3,73}$ & $\underline{42,33}$ & $\underline{7,18}$ \\
\hline 8 & 10,66 & 2,77 & 10,35 & 3,22 & 8,87 & 1,93 & 22,81 & 3,00 & 22,78 & 3,21 & 65,62 & 8,53 \\
\hline 9 & 10,17 & 2,08 & 7,90 & 1,23 & 12,76 & 2,84 & $\underline{20,69}$ & $\underline{2,15}$ & $\underline{41,43}$ & $\underline{4,30}$ & $\underline{81,01}$ & $\underline{7,90}$ \\
\hline 10 & 11,95 & 3,40 & 10,99 & 2,34 & 13,59 & 2,82 & $\underline{33,73}$ & $\underline{4,17}$ & $\underline{34,49}$ & $\underline{4,16}$ & $\underline{84,51}$ & $\underline{10,81}$ \\
\hline
\end{tabular}

Fonte: Fernando Carvalheiro Reiser (2019)

Post hoc: $\mathrm{p}<0.05$ em itálico/sublinhado

Na tabela 15 está o índice PMC-SAD, com médias e erro padrão do bloco, flecha e escore. Não houve efeito neste índice no APA5 para bloco $\left(F_{11,520}=1.3 ; \mathrm{p}=0.2\right)$, flecha $\left(F_{5,520}=1.4 ; \mathrm{p}=0.2\right)$ e escore $\left(F_{3,520}=0.3 ; \mathrm{p}=0.9\right)$. Não houve efeito neste índice no 
APA4 para bloco $\left(F_{11,520}=1.4 ; \mathrm{p}=0.2\right)$, flecha $\left(F_{5,520}=1.1 ; \mathrm{p}=0.35\right)$, e escore $\left(F_{3,520}=0.5\right.$; $\mathrm{p}=0.7)$. Não houve efeito neste índice no APA3 do bloco $\left(F_{11,520}=0.45 ; \mathrm{p}=0.9\right)$, flecha $\left(F_{5,520}=0.72 ; \mathrm{p}=0.61\right)$, e escore $\left(F_{3,520}=1.0 ; \mathrm{p}=0.4\right)$. Não houve efeito neste índice no APA2 para blocos $\left(F_{11,520}=1.4 ; \mathrm{p}=0.2\right)$, flechas $\left(F_{5,520}=0.6 ; \mathrm{p}=0.7\right)$, e escore $\left(F_{3,520}=0.3\right.$; $\mathrm{p}=0.83)$. Não houve efeito neste índice no APA1 para bloco $\left(F_{11,520}=1.8 ; \mathrm{p}=0.1\right)$, flecha $\left(F_{5,520}=0.1 ; \mathrm{p}=0.9\right)$, e escore $\left(F_{3,520}=1.8 ; \mathrm{p}=0.15\right)$. Não houve efeito neste índice no APC1 para blocos $\left(F_{11,520}=1.2 ; \mathrm{p}=0.3\right)$, flecha $\left(F_{5,520}=0.4 ; \mathrm{p}=0.8\right)$ e escore $\left(F_{3,520}=1.43\right.$; $\mathrm{p}=0.23)$.

Tabela 15. Co-ativação do par GD-TS com Média e EP

\begin{tabular}{|c|c|c|c|c|c|c|c|c|c|c|c|c|}
\hline \multirow[t]{2}{*}{ Ends } & \multicolumn{2}{|c|}{$\begin{array}{l}\text { GD-TS } \\
\text { (APA5) }\end{array}$} & \multicolumn{2}{|c|}{$\begin{array}{l}\text { GD-TS } \\
\text { (APA4) }\end{array}$} & \multicolumn{2}{|c|}{$\begin{array}{l}\text { GD-TS } \\
\text { (APA3) }\end{array}$} & \multicolumn{2}{|c|}{$\begin{array}{l}\text { GD-TS } \\
\text { (APA2) }\end{array}$} & \multicolumn{2}{|c|}{$\begin{array}{l}\text { GD-TS } \\
\text { (APA1) }\end{array}$} & \multicolumn{2}{|c|}{$\begin{array}{l}\text { GD-TS } \\
\text { (APC1) }\end{array}$} \\
\hline & Mean & SE & Mean & SE & Mean & SE & Mean & SE & Mean & $\mathrm{SE}$ & Mean & SE \\
\hline 1 & 7,33 & 0,71 & 7,71 & 0,69 & 8,28 & 1,03 & 10,43 & 1,26 & 54,61 & 15,72 & 74,63 & 20,03 \\
\hline 2 & 7,69 & 0,73 & 7,48 & 0,76 & 8,22 & 0,93 & 13,18 & 4,73 & 31,03 & 8,66 & 60,05 & 16,76 \\
\hline 3 & 11,09 & 1,73 & 11,31 & 1,74 & 11,23 & 1,70 & 12,50 & 1,71 & 42,39 & 9,18 & 83,06 & 17,65 \\
\hline 4 & 11,76 & 2,02 & 10,48 & 1,61 & 9,47 & 1,41 & 20,62 & 5,87 & 71,16 & 17,27 & 88,43 & 24,30 \\
\hline 5 & 10,92 & 1,35 & 12,16 & 1,90 & 10,59 & 1,33 & 12,87 & 1,59 & 66,17 & 16,84 & 70,69 & 18,29 \\
\hline 6 & 12,35 & 1,87 & 12,43 & 1,93 & 12,30 & 2,09 & 13,86 & 1,84 & 67,06 & 15,58 & 65,35 & 15,33 \\
\hline 7 & 15,09 & 4,11 & 19,13 & 7,40 & 12,61 & 3,03 & 14,10 & 3,12 & 37,50 & 9,73 & 42,34 & 13,12 \\
\hline 8 & 10,60 & 1,50 & 8,15 & 0,91 & 9,32 & 1,32 & 10,15 & 1,34 & 25,31 & 6,91 & 16,72 & 4,83 \\
\hline 9 & 9,00 & 1,20 & 11,01 & 1,53 & 11,90 & 1,53 & 12,05 & 1,72 & 32,55 & 9,66 & 43,06 & 12,91 \\
\hline 10 & 9,98 & 1,27 & 9,87 & 1,20 & 10,74 & 1,32 & 10,87 & 1,42 & 25,33 & 5,91 & 31,66 & 9,13 \\
\hline 11 & 8,85 & 1,39 & 9,98 & 1,25 & 9,29 & 1,15 & 12,19 & 1,86 & 43,81 & 12,16 & 49,53 & 14,35 \\
\hline 12 & 9,92 & 1,32 & 12,60 & 2,87 & 16,10 & 6,73 & 16,57 & 4,53 & 35,01 & 8,62 & 42,17 & 14,41 \\
\hline \multicolumn{13}{|l|}{ Arrow } \\
\hline 1 & 8,21 & 0,65 & 8,95 & 0,71 & 9,11 & 0,73 & 10,98 & 1,18 & 48,10 & 8,90 & 65,69 & 13,45 \\
\hline 2 & 8,68 & 0,72 & 9,07 & 0,76 & 8,09 & 0,57 & 10,27 & 1,05 & 42,39 & 8,68 & 58,08 & 12,12 \\
\hline 3 & 11,05 & 1,41 & 10,39 & 1,53 & 10,25 & 0,97 & 13,58 & 1,69 & 46,49 & 8,65 & 52,03 & 10,15 \\
\hline 4 & 9,69 & 0,92 & 11,45 & 1,65 & 13,25 & 3,47 & 13,41 & 2,13 & 45,09 & 7,96 & 57,83 & 11,49 \\
\hline 5 & 13,66 & 2,17 & 14,73 & 3,62 & 12,17 & 1,70 & 15,68 & 2,97 & 47,66 & 9,72 & 49,08 & 9,73 \\
\hline 6 & 11,01 & 1,16 & 11,58 & 1,27 & 12,15 & 1,36 & 15,76 & 2,86 & 36,23 & 7,15 & 51,13 & 10,42 \\
\hline \multicolumn{13}{|l|}{ Score } \\
\hline 7 & 11,48 & 1,59 & 12,01 & 2,70 & 10,67 & 1,24 & 12,34 & 1,12 & 56,25 & 9,37 & 76,02 & 12,24 \\
\hline 8 & 10,84 & 1,01 & 12,25 & 1,43 & 13,11 & 2,31 & 14,11 & 1,80 & 44,43 & 6,02 & 52,56 & 8,37 \\
\hline 9 & 9,66 & 0,74 & 9,51 & 0,69 & 8,92 & 0,61 & 12,09 & 1,52 & 45,09 & 6,26 & 55,15 & 7,65 \\
\hline 10 & 9,74 & 0,83 & 10,85 & 0,99 & 11,29 & 1,05 & 15,38 & 2,48 & 28,61 & 5,34 & 36,59 & 8,51 \\
\hline
\end{tabular}

Fonte: Fernando Carvalheiro Reiser (2019)

4.2.4 Índices mistos

Na tabela 16 está o índice SAD-SAnD, com médias e erro padrão do bloco, flecha e escore. Houve efeito no APA5 para bloco $\left(F_{11,520}=8.4 ; \mathrm{p}<0.01\right)$, maior no bloco 5 comparado ao 1-4,6-11 ( $<<0.01)$, não houve efeito nas flechas $\left(F_{5,520}=1.6 ; \mathrm{p}=0.2\right)$, Houve efeito nos escores $\left(F_{3,520}=3.4 ; \mathrm{p}=0.02\right)$ maior no 10 comparado ao $9(\mathrm{p}=0.05)$. No 
APA4 houve efeito para bloco, $\left(F_{11,520}=6.6 ; \mathrm{p}<0.01\right)$; maior no 5 comparado ao 1-4,6-11 $(\mathrm{p}<0.01)$, não houve efeito nas flechas $\left(F_{5,520}=1.7 ; \mathrm{p}=0.2\right)$ e escore $\left(F_{3,520}=1.7 ; \mathrm{p}=0.2\right)$.

No APA3 houve efeito para bloco $\left(F_{11,520}=2.7 ; \mathrm{p}<0.01\right)$; maior no 5 comparado ao 12 $(\mathrm{p}<0.01)$. Não houve efeito para flecha $\left(F_{5,520}=0.8 ; \mathrm{p}=0.6\right)$, e escore $\left(F_{3,520}=0.7 ; \mathrm{p}=0.6\right)$.

Neste índice houve efeito no APA2 para bloco, $\left(F_{11,520}=3.8 ; \mathrm{p}<0.01\right)$, no 5 e $1,2,4$ e 7 $(\mathrm{p}<0.02)$. Não houve efeito para flecha $\left(F_{5,520}=0.7 ; \mathrm{p}=0.7\right)$. Houve efeito nos escores $\left(F_{3,520}=4.1 ; \mathrm{p}<0.01\right)$; maior no 10 comparado ao 7 ( $\left.<<0.01\right)$ Não houve efeito no APA1: para bloco $\left(F_{11,520}=1.5 ; \mathrm{p}=0.1\right)$ e flecha $\left(F_{5,520}=0.6 ; \mathrm{p}=0.7\right)$. Houve efeito no escore $\left(F_{3,520}=5.6 ; \mathrm{p}<0.01\right)$; maior no 9 comparado ao 8 e 7 ( $\left.<<0.01\right)$. No APC1, não houve efeito nos Blocos $\left(F_{11,520}=1.7 ; \mathrm{p}=0.1\right)$ e flecha $\left(F_{5,520}=1.6 ; \mathrm{p}=0.2\right)$, contudo nos escores houve $\left(F_{3,520}=7.4 ; \mathrm{p}<0.01\right)$, maior no 10 e 9 comparados ao 7 e $8(\mathrm{p}<0.01)$.

Tabela 16. Co-ativação do par SAD-SAnD com Média e EP

\begin{tabular}{|c|c|c|c|c|c|c|c|c|c|c|c|c|}
\hline \multirow[t]{2}{*}{ Ends } & \multicolumn{2}{|c|}{$\begin{array}{l}\text { SAD-SAnDcc } \\
\text { (APA5) }\end{array}$} & \multicolumn{2}{|c|}{$\begin{array}{l}\text { SAD-SAnD } \\
\text { (APA4) }\end{array}$} & \multicolumn{2}{|c|}{$\begin{array}{l}\text { SAD-SAnD } \\
\text { (APA3) }\end{array}$} & \multicolumn{2}{|c|}{$\begin{array}{l}\text { SAD-SAnD } \\
\text { (APA2) }\end{array}$} & \multicolumn{2}{|c|}{$\begin{array}{l}\text { SAD-SAnD } \\
\text { (APA1) }\end{array}$} & \multicolumn{2}{|c|}{$\begin{array}{l}\text { SAD-SAnD } \\
\text { (APC1) }\end{array}$} \\
\hline & Mean & SE & Mean & SE & Mean & SE & Mean & SE & Mean & SE & Mean & $\mathrm{SE}$ \\
\hline $\mathbf{1}$ & $\underline{3,71}$ & $\underline{0,45}$ & 4,47 & $\underline{0,60}$ & 4,47 & 0,55 & $\underline{6,75}$ & $\underline{0,92}$ & 22,88 & 5,28 & 33,07 & 8,97 \\
\hline 2 & $\underline{4,69}$ & $\underline{0,62}$ & $\underline{4,18}$ & $\underline{0,39}$ & 5,22 & 0,97 & $\underline{8,83}$ & $\underline{2,30}$ & 20,91 & 4,10 & 23,09 & 6,93 \\
\hline 3 & $\underline{5,53}$ & $\underline{0,79}$ & $\underline{5,33}$ & $\underline{0,61}$ & 6,42 & 1,11 & 13,62 & 2,68 & 33,68 & 8,15 & 32,92 & 6,57 \\
\hline 4 & $\underline{5,32}$ & $\underline{0,72}$ & $\underline{4,30}$ & $\underline{0,52}$ & 5,18 & 0,57 & $\underline{8,50}$ & $\underline{0,81}$ & 32,63 & 7,82 & 29,42 & 7,76 \\
\hline 5 & $\underline{26,56}$ & $\underline{8,71}$ & $\underline{18,81}$ & $\underline{7,64}$ & $\underline{19,32}$ & $\underline{7,08}$ & $\underline{20,78}$ & $\underline{4,92}$ & 44,00 & 8,27 & 47,66 & 9,72 \\
\hline 6 & $\underline{8,74}$ & 3,91 & 4,84 & $\underline{0,46}$ & 4,66 & 0,43 & $\overline{12,26}$ & 1,59 & 38,79 & 8,56 & 28,50 & 6,83 \\
\hline 7 & $\underline{3,87}$ & $\underline{0,46}$ & $\underline{5,15}$ & $\underline{0,63}$ & 4,88 & 0,53 & $\underline{8,79}$ & $\underline{1,05}$ & 25,12 & 6,16 & 20,62 & 5,32 \\
\hline 8 & $\overline{4,29}$ & $\overline{0,54}$ & $\overline{5,02}$ & $\overline{0,63}$ & 4,56 & 0,56 & $\overline{11,26}$ & $\overline{2,01}$ & 30,88 & 5,60 & 34,80 & 8,81 \\
\hline 9 & $\underline{4,47}$ & $\underline{0,57}$ & $\underline{5,18}$ & $\underline{0,64}$ & 12,14 & 5,91 & 12,39 & 3,25 & 16,69 & 3,13 & 8,39 & 9,07 \\
\hline 10 & $\underline{4,01}$ & $\underline{0,39}$ & $\underline{4,48}$ & $\underline{0,46}$ & 4,87 & 0,50 & 12,00 & 1,84 & 38,41 & 8,23 & 29,96 & 7,92 \\
\hline 11 & $\underline{4,06}$ & $\underline{0,39}$ & $\underline{5,00}$ & $\underline{0,73}$ & 10,59 & 5,87 & 11,73 & 2,48 & 33,14 & 8,57 & 29,69 & 9,45 \\
\hline 12 & 13,30 & 5,39 & $\underline{5,92}$ & $\underline{1,26}$ & $\underline{4,07}$ & $\underline{0,42}$ & 10,68 & 1,41 & 33,59 & 7,43 & 29,36 & 8,28 \\
\hline \multicolumn{13}{|l|}{ Arrow } \\
\hline 1 & 7,35 & 2,47 & 7,87 & 3,25 & 10,26 & 3,74 & 12,34 & 2,34 & 26,58 & 4,41 & 20,87 & 3,69 \\
\hline 2 & 9,12 & 3,44 & 6,75 & 1,89 & 6,90 & 2,41 & 9,75 & 1,36 & 28,58 & 5,19 & 30,52 & 5,90 \\
\hline 3 & 5,56 & 1,23 & 5,19 & 0,46 & 5,62 & 1,13 & 11,15 & 1,36 & 34,82 & 4,94 & 41,41 & 7,47 \\
\hline 4 & 9,45 & 2,80 & 4,78 & 0,40 & 4,70 & 0,37 & 9,37 & 0,84 & 30,56 & 5,09 & 28,89 & 5,63 \\
\hline 5 & 6,70 & 1,67 & 5,51 & 0,69 & 9,79 & 3,07 & 13,06 & 1,96 & 33,81 & 5,64 & 22,67 & 4,18 \\
\hline 6 & 6,09 & 1,49 & 6,23 & 1,17 & 5,92 & 0,82 & 13,12 & 1,89 & 31,01 & 4,48 & 39,38 & 6,30 \\
\hline \multicolumn{13}{|l|}{ Score } \\
\hline 7 & 9,83 & 2,64 & 6,36 & 1,05 & 5,78 & 0,80 & $\underline{8,54}$ & $\underline{1,07}$ & $\underline{21,52}$ & $\underline{3,73}$ & $\underline{15,79}$ & $\underline{3,12}$ \\
\hline 8 & 7,25 & 1,76 & 7,00 & 2,09 & 7,19 & 1,66 & $\underline{11,03}$ & $\underline{1,45}$ & $\underline{22,78}$ & $\underline{3,21}$ & $\underline{22,53}$ & $\underline{4,01}$ \\
\hline 9 & $\underline{4,07}$ & $\underline{0,27}$ & 4,68 & 0,30 & 7,43 & 2,08 & 11,15 & 1,00 & $\underline{41,43}$ & $\underline{4,30}$ & $\underline{40,43}$ & $\underline{4,80}$ \\
\hline 10 & $\underline{10,59}$ & $\underline{3,11}$ & 6,85 & 1,64 & 8,48 & 2,25 & $\underline{16,13}$ & $\underline{2,19}$ & 34,49 & 4,16 & $\underline{42,01}$ & $\underline{5,77}$ \\
\hline
\end{tabular}

Fonte: Fernando Carvalheiro Reiser (2019)

Post hoc: $\mathrm{p}<0.05$ em itálico/sublinhado

Na tabela 17 está o índice SAnD-DPnD, com médias e erro padrão do bloco, flecha e escore. Houve efeito no APA5 do bloco $\left(F_{11,520}=3.0 ; \mathrm{p}<0.01\right)$; maior 5 
comparado a 1,2,7,10-12 ( $\mathrm{p}<0.05)$. Não houve efeito da flecha $\left(F_{5,520}=0.6 ; \mathrm{p}=0.7\right)$. Houve efeito do escore $\left(F_{3,520}=4.8 ; \mathrm{p}<0.01\right)$ maior no 10 comparado ao 7 e 9 ( $\left.<<0.04\right)$. Não houve efeito no APA4 do bloco $\left(F_{11,520}=1.6 ; \mathrm{p}=0.1\right)$ e flecha $\left(F_{5,520}=0.8 ; \mathrm{p}=0.5\right)$. Houve efeito do escore $\left(F_{3,520}=3.5 ; \mathrm{p}<0.01\right)$, maior no escore 10 em comparação ao 9 $(\mathrm{p}=0.05)$. No APA3, houve efeito do bloco $\left(F_{11,520}=2.0 ; \mathrm{p}=0.03\right)$, sem detecção no post hoc. Não houve efeito da flecha $\left(F_{5,520}=1.1 ; \mathrm{p}=0.4\right)$. Houve efeito do escore $\left(F_{3,520}=4.3\right.$; $\mathrm{p}<0.01)$ maior no 10 comparado ao $9(\mathrm{p}<0.01)$. No APA2, não houve efeito do bloco $\left(F_{11,520}=1.7 ; \mathrm{p}=0.1\right)$, flecha $\left(F_{5,520}=0.6 ; \mathrm{p}=0.7\right)$ e escore $\left(F_{3,520}=2.2 \mathrm{p}=0.1\right)$. No APA1, não houve efeito do bloco $\left(F_{11,520}=0.8 ; \mathrm{p}=0.7\right)$, flecha $\left(F_{5,520}=0.5 ; \mathrm{p}=0.8\right)$ e escore $\left(F_{3,520}=0.7 ; \mathrm{p}=0.5\right)$. Não houve efeito no APC1 do bloco $\left(F_{11,520}=0.5 ; \mathrm{p}=0.9\right)$ e flecha $\left(F_{5,520}=0.7 ; \mathrm{p}=0.6\right)$, mas houve do escore $\left(F_{3,520}=3.0 ; \mathrm{p}=0.03\right)$ maior no 8 em comparação ao $9(\mathrm{p}=0.05)$.

Tabela 17. Co-ativação do par SAnD-DPnD com Média e EP

\begin{tabular}{|c|c|c|c|c|c|c|c|c|c|c|c|c|}
\hline \multirow[t]{2}{*}{ Ends } & \multicolumn{2}{|c|}{$\begin{array}{c}\text { SAnD-DPnD } \\
\text { (APA5) }\end{array}$} & \multicolumn{2}{|c|}{$\begin{array}{c}\text { SAnD-DPnD } \\
\text { (APA4) }\end{array}$} & \multicolumn{2}{|c|}{$\begin{array}{c}\text { SAnD-DPnD } \\
\text { (APA3) }\end{array}$} & \multicolumn{2}{|c|}{$\begin{array}{c}\text { SAnD-DPnD } \\
\text { (APA2) }\end{array}$} & \multicolumn{2}{|c|}{$\begin{array}{c}\text { SAnD-DPnD } \\
\text { (APA1) }\end{array}$} & \multicolumn{2}{|c|}{$\begin{array}{l}\text { SAnD-DPnD } \\
\text { (APC1) }\end{array}$} \\
\hline & Mean & SE & Mean & SE & Mean & SE & Mean & SE & Mean & SE & Mean & SE \\
\hline 1 & 9,20 & $\underline{1,77}$ & 12,35 & 4,82 & 10,73 & 3,25 & 13,05 & 3,63 & 29,30 & 6,69 & 24,55 & 6,39 \\
\hline 2 & 12,62 & $\underline{2,85}$ & 14,07 & 2,98 & 21,26 & 6,14 & 16,02 & 4,09 & 32,53 & 7,32 & 35,15 & 7,04 \\
\hline 3 & 15,17 & 4,13 & 12,03 & 2,59 & 13,07 & 2,78 & 16,62 & 3,22 & 36,71 & 10,14 & 38,69 & 8,40 \\
\hline 4 & 16,84 & 5,19 & 16,68 & 4,64 & 21,06 & 6,85 & 20,08 & 4,66 & 28,14 & 6,82 & 37,68 & 8,33 \\
\hline 5 & $\underline{31,35}$ & $\underline{8,88}$ & 24,77 & 7,62 & 24,96 & 7,01 & 23,78 & 4,94 & 44,10 & 8,12 & 48,15 & 11,27 \\
\hline 6 & 13,27 & 3,71 & 10,42 & 1,58 & 12,34 & 2,79 & 16,60 & 2,24 & 40,31 & 8,22 & 37,25 & 6,99 \\
\hline 7 & $\underline{8,80}$ & $\underline{1,32}$ & 11,08 & 1,65 & 11,76 & 2,07 & 14,12 & 2,28 & 35,80 & 8,05 & 27,43 & 6,54 \\
\hline 8 & 13,28 & 3,85 & 16,48 & 6,13 & 19,82 & 6,89 & 17,70 & 4,37 & 26,76 & 5,94 & 41,13 & 9,00 \\
\hline 9 & 13,03 & 3,08 & 16,13 & 5,21 & 14,55 & 3,74 & 17,89 & 4,62 & 23,52 & 4,98 & 37,98 & 10,18 \\
\hline 10 & $\underline{12,52}$ & $\underline{4,01}$ & 11,30 & 3,28 & 11,47 & 2,90 & 13,72 & 2,55 & 28,39 & 6,52 & 34,84 & 6,74 \\
\hline 11 & $\underline{8,23}$ & 1,14 & 8,38 & 1,21 & 8,32 & 1,12 & 10,94 & 1,30 & 33,19 & 7,25 & 31,74 & 7,72 \\
\hline 12 & $\underline{12,05}$ & 4,31 & 14,52 & 4,59 & 13,14 & 4,35 & 11,61 & 1,49 & 32,58 & 7,45 & 32,97 & 7,92 \\
\hline \multicolumn{13}{|l|}{ Arrow } \\
\hline 1 & 15,94 & 3,60 & 15,10 & 4,00 & 16,50 & 4,07 & 17,81 & 3,25 & 29,11 & 5,16 & 32,31 & 6,29 \\
\hline 2 & 12,74 & 3,08 & 17,16 & 3,61 & 17,71 & 3,96 & 14,82 & 2,41 & 28,02 & 4,86 & 31,42 & 5,13 \\
\hline 3 & 14,84 & 3,33 & 12,22 & 2,47 & 13,51 & 2,65 & 14,41 & 1,62 & 32,46 & 5,04 & 28,38 & 4,98 \\
\hline 4 & 13,62 & 2,86 & 15,40 & 2,86 & 16,06 & 2,93 & 17,21 & 2,47 & 37,80 & 6,13 & 38,40 & 5,94 \\
\hline 5 & 12,58 & 2,51 & 10,69 & 1,61 & 14,32 & 3,04 & 15,42 & 2,57 & 34,14 & 5,33 & 37,57 & 5,59 \\
\hline 6 & 13,47 & 2,34 & 13,54 & 3,12 & 13,14 & 2,66 & 16,40 & 2,32 & 34,13 & 4,72 & 45,70 & 6,46 \\
\hline \multicolumn{13}{|l|}{ Score } \\
\hline 7 & $\underline{11,47}$ & $\underline{2,02}$ & 11,57 & 1,97 & 13,04 & 2,56 & 15,29 & 2,43 & 30,61 & 4,37 & 31,34 & 4,74 \\
\hline 8 & 15,54 & 2,89 & 16,35 & 3,30 & 17,40 & 2,95 & 17,23 & 2,14 & 38,92 & 4,70 & $\underline{44,69}$ & $\underline{5,51}$ \\
\hline 9 & $\underline{10,00}$ & $\underline{1,20}$ & $\underline{10,66}$ & $\underline{1,52}$ & $\underline{10,74}$ & $\underline{1,22}$ & 13,41 & 1,37 & 30,08 & 3,77 & $\underline{28,43}$ & $\underline{3,32}$ \\
\hline 10 & $\underline{21,30}$ & $\underline{3,95}$ & $\underline{19,70}$ & $\underline{3,17}$ & $\underline{22,73}$ & $\underline{4,46}$ & 19,83 & 2,50 & 30,69 & 3,92 & 40,97 & 5,70 \\
\hline
\end{tabular}


Na tabela 18 está o índice GD-DPnD, com médias e erro padrão do bloco, flechas e escores. Não houve efeito no APA5 do bloco $\left(F_{11,520}=1.0 ; \mathrm{p}=0.5\right)$, e flecha $\left(F_{5,520}=1.0 ; \mathrm{p}=0.4\right)$ neste índice, e houve no escore $\left(F_{3,520}=4.1 ; \mathrm{p}<0.01\right)$, maior no 10 comparado a 9 e 7 ( $<<0.02)$. Não houve efeito no APA4 do bloco $\left(F_{11,520}=0.7 ; \mathrm{p}=0.8\right)$, flecha $(F 5,520=0.9 ; \mathrm{p}=0.5)$ e escore $\left(F_{3,520}=2.4 ; \mathrm{p}=0.1\right)$. Não houve efeito no APA3 do bloco $\left(F_{11,520}=1.5 ; \mathrm{p}=0.1\right)$, e flecha $\left(F_{5,520}=1.0 ; \mathrm{p}=0.4\right)$, e houve no escore $\left(F_{3,520}=3.2\right.$; p=0.02), maior no 10 em comparação ao 9 ( $\mathrm{p}<0.02)$. Não houve efeito no APA2 do bloco $\left(F_{11,520}=1.1 ; \mathrm{p}=0.4\right)$, flecha $\left(F_{5,520}=0.7 ; \mathrm{p}=0.6\right)$ e escore $\left(F_{3,520}=1.7 ; \mathrm{p}=0.2\right)$. Não houve efeito no APA1 do bloco $\left(F_{11,520}=0.5 ; \mathrm{p}=0.9\right)$, flecha $\left(F_{5,520}=0.3 ; \mathrm{p}=0.9\right)$ e escore $\left(F_{3,520}=1.3 ; \mathrm{p}=0.2\right)$. Não houve efeito no $\mathrm{APC} 1$ do bloco $\left(F_{11,520}=0.5 ; \mathrm{p}=0.92\right)$, flecha $\left(F_{5,520}=0.8 ; \mathrm{p}=0.6\right)$ e escore $\left(F_{3,520}=1.7 ; \mathrm{p}=0.2\right)$.

Tabela 18. Co-ativação do par GD-DPnD com Média e EP

\begin{tabular}{|c|c|c|c|c|c|c|c|c|c|c|c|c|}
\hline \multirow[t]{2}{*}{ Ends } & \multicolumn{2}{|c|}{$\begin{array}{c}\text { GD-DPnD } \\
\text { (APA5) }\end{array}$} & \multicolumn{2}{|c|}{$\begin{array}{c}\text { GD-DPnD } \\
\text { (APA4) }\end{array}$} & \multicolumn{2}{|c|}{$\begin{array}{c}\text { GD-DPnD } \\
\text { (APA3) }\end{array}$} & \multicolumn{2}{|c|}{$\begin{array}{l}\text { GD-DPnD } \\
\text { (APA2) }\end{array}$} & \multicolumn{2}{|c|}{$\begin{array}{c}\text { GD-DPnD } \\
\text { (APA1) }\end{array}$} & \multicolumn{2}{|c|}{$\begin{array}{l}\text { GD-DPnD } \\
\text { (APC1) }\end{array}$} \\
\hline & Mean & SE & Mean & SE & Mean & SE & Mean & SE & Mean & SE & Mean & SE \\
\hline 1 & 9,55 & 1,71 & 12,76 & 4,73 & 11,11 & 3,20 & 12,10 & 3,55 & 29,73 & 6,94 & 37,54 & 10,49 \\
\hline 2 & 12,95 & 2,77 & 14,65 & 2,91 & 21,71 & 6,06 & 15,15 & 4,00 & 33,21 & 7,71 & 42,90 & 9,15 \\
\hline 3 & 15,66 & 4,08 & 12,63 & 2,57 & 13,72 & 2,75 & 15,02 & 3,05 & 45,88 & 11,32 & 70,89 & 15,89 \\
\hline 4 & 17,33 & 5,20 & 17,21 & 4,57 & 21,35 & 6,72 & 18,97 & 4,52 & 31,86 & 7,27 & 49,43 & 9,47 \\
\hline 5 & 13,51 & 3,67 & 11,94 & 1,78 & 11,78 & 1,57 & 12,34 & 1,60 & 34,82 & 8,01 & 43,68 & 8,48 \\
\hline 6 & 14,06 & 3,71 & 11,25 & 1,60 & 12,91 & 2,72 & 14,05 & 1,92 & 39,59 & 8,87 & 40,34 & 8,06 \\
\hline 7 & 11,15 & 2,21 & 14,19 & 2,97 & 13,08 & 2,22 & 16,97 & 3,40 & 41,10 & 9,53 & 34,69 & 8,23 \\
\hline 8 & 13,80 & 3,80 & 17,12 & 6,09 & 20,48 & 6,87 & 16,32 & 4,33 & 27,75 & 6,43 & 40,36 & 8,86 \\
\hline 9 & 13,60 & 3,04 & 16,70 & 5,18 & 15,01 & 3,68 & 17,69 & 4,61 & 32,47 & 8,27 & 51,69 & 11,99 \\
\hline 10 & 13,23 & 3,94 & 12,07 & 3,25 & 12,06 & 2,86 & 12,84 & 2,40 & 32,44 & 7,28 & 42,91 & 8,27 \\
\hline 11 & 8,54 & 1,10 & 8,95 & 1,17 & 8,54 & 1,09 & 11,23 & 1,59 & 40,76 & 9,88 & 46,58 & 11,41 \\
\hline 12 & 13,37 & 4,37 & 15,18 & 4,52 & 14,14 & 4,32 & 10,74 & 1,39 & 32,72 & 8,33 & 39,08 & 11,02 \\
\hline \multicolumn{13}{|l|}{ Arrow } \\
\hline 1 & 13,84 & 2,88 & 12,31 & 2,41 & 14,43 & 3,35 & 15,73 & 2,87 & 31,81 & 5,72 & 38,37 & 7,17 \\
\hline 2 & 10,53 & 1,36 & 15,87 & 3,15 & 15,67 & 3,21 & 12,67 & 2,10 & 29,52 & 5,59 & 44,82 & 7,77 \\
\hline 3 & 15,04 & 3,24 & 13,52 & 2,71 & 13,37 & 2,46 & 13,79 & 1,70 & 36,12 & 5,95 & 39,56 & 6,52 \\
\hline 4 & 14,03 & 2,85 & 15,98 & 2,83 & 16,58 & 2,88 & 16,05 & 2,42 & 40,16 & 6,65 & 49,86 & 7,98 \\
\hline 5 & 12,19 & 2,03 & 11,52 & 1,55 & 14,59 & 2,94 & 14,89 & 2,54 & 38,82 & 6,17 & 45,46 & 6,63 \\
\hline 6 & 12,73 & 1,86 & 13,13 & 2,93 & 13,30 & 2,58 & 13,58 & 1,94 & 34,73 & 5,52 & 51,98 & 7,69 \\
\hline \multicolumn{13}{|l|}{ Score } \\
\hline 7 & $\underline{10,25}$ & $\underline{1,32}$ & 11,20 & 1,77 & 12,75 & 2,45 & 14,51 & 2,32 & 34,65 & 5,52 & 42,90 & 6,77 \\
\hline 8 & 14,39 & 2,51 & 15,63 & 2,70 & 16,11 & 2,48 & 15,43 & 1,88 & 43,27 & 5,16 & 52,97 & 6,24 \\
\hline 9 & $\underline{10,59}$ & $\underline{1,17}$ & 11,18 & 1,50 & $\underline{11,11}$ & $\underline{1,19}$ & 11,91 & 1,31 & 30,23 & 3,94 & 37,35 & 4,45 \\
\hline 10 & $\underline{19,00}$ & $\underline{3,10}$ & 18,60 & 2,77 & 21,26 & $\underline{3,95}$ & 17,59 & 2,29 & 33,44 & 4,96 & 50,11 & 7,22 \\
\hline
\end{tabular}


Para os APA e APC, o índice FSD-ED foi comparado entre blocos, flechas e escores (tabela 19). No APA5, esse índice não sofreu efeito do bloco, $\left(F_{11,520}=0.2\right.$, $\mathrm{p}=0.9)$; flecha $\left(F_{5,520}=0.5, \mathrm{p}=0.8\right)$ e escore $\left(F_{3,520}=0.6, \mathrm{p}=0.6\right)$. No APA4, esse índice não sofreu efeito do bloco, $\left(F_{11,520}=1.1, \mathrm{p}=0.4\right)$, flecha $\left(F_{5,520}=0.8, \mathrm{p}=0.6\right)$ e escore $\left(F_{3,520}=1.6, \mathrm{p}=0.2\right)$. No APA3, não teve efeito do bloco $\left(F_{11,520}=0.5, \mathrm{p}=0.9\right)$; flechas $\left(F_{5,520}=0.6, \mathrm{p}=0.8\right)$ e escore $\left(F_{3,520}=2.0, \mathrm{p}=0.1\right)$. No APA2, esse índice sofreu efeito do bloco $(F 11,520=2.2, \mathrm{p}=0.01)$; com maior módulo no bloco 3 comparado ao 1,7,9 e 10 ( $\mathrm{p}<0,05)$, não houve efeito da flecha $\left(F_{5,520}=0.9, \mathrm{p}=0.5\right)$, e houve no escore $\left(F_{3,520}=3.3\right.$, $\mathrm{p}<0.02$ ), com maior módulo no 9 comparado ao 7 ( $\mathrm{p}<0.04$ ). No APA1, houve efeito do bloco $\left(F_{11,520}=2.0, \mathrm{p}=0.5\right)$, com maior módulo no bloco 3 em comparação com o $1,7,9,10$ e 12 (p<0,05). Não houve efeito da flecha $\left(F_{5,520}=0.7, \mathrm{p}=0.6\right)$ e escore $\left(F_{3,520}=1.6, \mathrm{p}=0.2\right)$. No APC1, não houve efeito do bloco $(F 11,520=2.0, \mathrm{p}=0.3)$ e da flecha $\left(F_{5,520}=0.5, \mathrm{p}=0.8\right)$, e houve no escore $\left(F_{3,520}=4.0, \mathrm{p}<0.01\right)$; quando houve maior índice no escore 10 e 9 comparado ao com 7 ( $\mathrm{p}<0.05)$.

Tabela 19. Inibição reciproca do par FSD-ED com Média e EP

\begin{tabular}{|c|c|c|c|c|c|c|c|c|c|c|c|c|}
\hline \multirow[t]{2}{*}{ Ends } & \multicolumn{2}{|c|}{$\begin{array}{c}\text { FDS-ED } \\
\text { (APA5) }\end{array}$} & \multicolumn{2}{|c|}{$\begin{array}{c}\text { FDS-ED } \\
\text { (APA4) }\end{array}$} & \multicolumn{2}{|c|}{$\begin{array}{c}\text { FDS-ED } \\
\text { (APA3) }\end{array}$} & \multicolumn{2}{|c|}{$\begin{array}{c}\text { FDS-ED } \\
\text { (APA2) }\end{array}$} & \multicolumn{2}{|c|}{$\begin{array}{c}\text { FDS-ED } \\
\text { (APA1) }\end{array}$} & \multicolumn{2}{|c|}{$\begin{array}{c}\text { FDS-ED } \\
\text { (APC1) }\end{array}$} \\
\hline & Mean & SE & Mean & SE & Mean & SE & Mean & $\mathrm{SE}$ & Mean & SE & Mean & SE \\
\hline 1 & 2,60 & 0,66 & 3,61 & 1,17 & 3,67 & 1,10 & 7,13 & $\underline{1,38}$ & 41,06 & 9,33 & 31,80 & 10,78 \\
\hline 2 & 4,00 & 1,03 & 4,46 & 1,24 & 4,13 & 1,28 & 13,57 & 2,90 & 88,53 & 15,24 & 59,15 & 15,03 \\
\hline 3 & 3,11 & 0,54 & 4,30 & 1,16 & 4,20 & 1,04 & $\underline{20,10}$ & $\underline{3,46}$ & $\underline{124,88}$ & $\underline{27,32}$ & 97,72 & 22,20 \\
\hline 4 & 2,81 & 0,55 & 3,92 & 0,94 & 3,82 & 0,79 & 8,93 & 1,38 & 87,50 & 18,36 & 45,40 & 12,22 \\
\hline 5 & 2,86 & 0,63 & 2,34 & 0,46 & 3,15 & 0,64 & 9,62 & 2,08 & 78,80 & 21,60 & 56,97 & 15,59 \\
\hline 6 & 2,73 & 0,44 & 2,82 & 0,53 & 3,22 & 0,57 & 10,06 & 2,02 & 66,79 & 14,50 & 57,06 & 16,56 \\
\hline 7 & 2,44 & 0,34 & 2,92 & 0,46 & 3,79 & 0,79 & 7,47 & 1,06 & 63,12 & 15,66 & 70,77 & 16,75 \\
\hline 8 & 2,85 & 0,45 & 2,33 & 0,35 & 3,07 & 0,44 & 10,56 & 6,49 & $\underline{40,34}$ & $\underline{13,10}$ & 43,20 & 13,09 \\
\hline 9 & 3,13 & 0,59 & 3,36 & 0,59 & 3,38 & 0,68 & $\underline{7,60}$ & $\underline{1,13}$ & $\underline{40,86}$ & $\underline{10,11}$ & 26,54 & 7,54 \\
\hline 10 & 3,06 & 0,49 & 2,31 & 0,34 & 2,44 & 0,39 & $\underline{4,61}$ & $\underline{0,81}$ & $\underline{36,26}$ & $\underline{9,15}$ & 40,63 & 10,65 \\
\hline 11 & 2,90 & 0,58 & 2,85 & 0,54 & 2,83 & 0,78 & 10,82 & 2,03 & 68,91 & 13,90 & 59,93 & 12,68 \\
\hline 12 & 2,78 & 0,60 & 1,90 & 0,30 & 2,45 & 0,31 & 9,25 & 2,19 & 43,38 & $\underline{9,93}$ & 35,81 & 10,70 \\
\hline \multicolumn{13}{|l|}{ Arrow } \\
\hline 1 & 2,51 & 0,28 & 2,93 & 0,58 & 3,18 & 0,58 & 9,91 & 3,38 & 51,15 & 9,56 & 41,12 & 8,70 \\
\hline 2 & 3,61 & 0,65 & 3,37 & 0,73 & 3,34 & 0,67 & 8,86 & 1,38 & 58,41 & 11,32 & 49,87 & 9,61 \\
\hline 3 & 2,76 & 0,39 & 3,38 & 0,61 & 3,83 & 0,61 & 10,59 & 1,60 & 69,50 & 12,66 & 42,12 & 8,66 \\
\hline 4 & 2,69 & 0,30 & 2,76 & 0,36 & 3,04 & 0,49 & 9,63 & 1,23 & 73,44 & 11,23 & 56,80 & 11,14 \\
\hline 5 & 3,22 & 0,43 & 3,40 & 0,47 & 3,24 & 0,39 & 10,67 & 1,65 & 72,30 & 12,67 & 60,82 & 10,49 \\
\hline 6 & 2,85 & 0,38 & 2,70 & 0,34 & 3,45 & 0,56 & 10,21 & 1,43 & 65,40 & 9,96 & 61,77 & 11,45 \\
\hline \multicolumn{13}{|l|}{ Score } \\
\hline 7 & 2,88 & 0,34 & 2,43 & 0,24 & 2,36 & 0,34 & $\underline{6,86}$ & $\underline{0,84}$ & 56,22 & 8,79 & 28,39 & $\underline{5,27}$ \\
\hline 8 & 3,25 & 0,45 & 3,56 & 0,52 & 3,39 & 0,47 & 9,80 & 2,25 & 52,97 & 7,68 & 47,98 & 8,03 \\
\hline 9 & 2,86 & 0,26 & 2,84 & 0,27 & 3,50 & 0,36 & $\underline{12,06}$ & $\underline{1,26}$ & 80,49 & 9,70 & $\underline{57,79}$ & $\underline{7,44}$ \\
\hline 10 & 2,72 & 0,29 & 3,68 & 0,72 & 4,18 & 0,67 & 10,19 & 1,26 & 64,68 & 9,17 & $\underline{75,78}$ & $\underline{11,75}$ \\
\hline
\end{tabular}

Fonte: Fernando Carvalheiro Reiser (2019)

Post hoc: $\mathrm{p}<0.05$ em itálico/sublinhado 
Na tabela 20, está o índice BBL-TBL. Neste índice não houve efeito no APA5 do bloco $\left(F_{11,520}=1.3 ; \mathrm{p}=0.3\right)$, flecha $\left(F_{5,520}=0.6 ; \mathrm{p}=0.7\right)$, e escore $\left(F_{3,520}=1.0 ; \mathrm{p}=0.4\right)$. No APA4, não houve efeito do bloco $\left(F_{11,520}=1.4 ; \mathrm{p}=0.2\right)$, flecha $\left(F_{5,520}=0.1 ; \mathrm{p}=0.9\right) \mathrm{e}$ escore $\left(F_{3,520}=1.2 ; \mathrm{p}=0.3\right)$. No APA3, houve efeito do bloco $\left(F_{11,520}=2.4 ; \mathrm{p}<0.01\right)$ maior no 4 comparado a 1-3,6,8-12, não houve da flecha $\left(F_{5,520}=1.5 ; \mathrm{p}=0.2\right)$, e houve do escore $\left(F_{3,520}=4.8 ; \mathrm{p}<0.01\right)$, maior no 10 em comparação com 7 e $8(\mathrm{p}<0.02)$. No APA2, não houve efeito do bloco $\left(F_{11,520}=1.4 ; \mathrm{p}=0.1\right)$ e flecha $\left(F_{5,520}=0.2 ; \mathrm{p}=0.9\right)$, e houve do escore $\left(F_{3,520}=6.2 ; \mathrm{p}<0.01\right)$; maior no 10 comparado ao 7,8 e $9(\mathrm{p}<0.03)$. Não houve efeito no APA1 do bloco $\left(F_{11,520}=1.4 ; \mathrm{p}=0.2\right)$ e flecha $\left(F_{5,520}=0.8 ; \mathrm{p}=0.5\right)$, e houve do escore $\left(F_{3,520}=3.8 ; \mathrm{p}<0.01\right)$, maior no 10 e 9 comparado ao $7(\mathrm{p}<0.05)$. Não houve efeito no APC1 do bloco $\left(F_{11,520}=1.6 ; \mathrm{p}=0.1\right)$ e flecha $\left(F_{5,520}=0.5 ; \mathrm{p}=0.8\right)$, e houve efeito nos escores $\left(F_{3,520}=4.8 ; \mathrm{p}<0.01\right)$; maior no 10 e 9 comparado ao $7(\mathrm{p}<0.05)$.

Tabela 20. Inibição reciproca do par BBL-TBL com Média e EP

\begin{tabular}{|c|c|c|c|c|c|c|c|c|c|c|c|c|}
\hline \multirow[t]{2}{*}{ Ends } & \multicolumn{2}{|c|}{$\begin{array}{c}\text { BBL-TBL } \\
\text { (APA5) }\end{array}$} & \multicolumn{2}{|c|}{$\begin{array}{c}\text { BBL-TBL } \\
\text { (APA4) }\end{array}$} & \multicolumn{2}{|c|}{$\begin{array}{c}\text { BBL-TBL } \\
\text { (APA3) }\end{array}$} & \multicolumn{2}{|c|}{$\begin{array}{c}\text { BBL-TBL } \\
\text { (APA2) }\end{array}$} & \multicolumn{2}{|c|}{$\begin{array}{c}\text { BBL-TBL } \\
\text { (APA1) }\end{array}$} & \multicolumn{2}{|c|}{$\begin{array}{c}\text { BBL-TBL } \\
\text { (APC1) }\end{array}$} \\
\hline & Mean & SE & Mean & SE & Mean & SE & Mean & SE & Mean & SE & Mean & SE \\
\hline 1 & 3,57 & 0,51 & 3,65 & 0,52 & $\underline{4,02}$ & $\underline{0,50}$ & 9,20 & 1,92 & 44,55 & 9,53 & 31,44 & 7,32 \\
\hline 2 & 3,76 & 0,68 & 4,67 & 0,77 & $\underline{5,19}$ & $\underline{0,87}$ & 14,76 & 4,81 & 37,62 & 10,06 & 25,21 & 7,53 \\
\hline 3 & 5,51 & 1,15 & 6,13 & 1,03 & 6,16 & $\underline{1,11}$ & 7,47 & 1,88 & 32,66 & 12,68 & 62,80 & 15,99 \\
\hline 4 & 13,61 & 5,12 & 18,23 & 7,48 & $\underline{22,41}$ & $\underline{11,49}$ & 18,14 & 8,05 & 72,93 & 21,52 & 75,62 & 19,04 \\
\hline 5 & 6,58 & 2,23 & 8,88 & 3,30 & 10,61 & 4,12 & 9,03 & 2,23 & 58,20 & 17,96 & 61,25 & 16,91 \\
\hline 6 & 5,95 & 1,34 & 4,74 & 0,76 & $\underline{5,48}$ & $\underline{0,89}$ & 7,78 & 1,56 & 65,57 & 18,79 & 58,34 & 15,30 \\
\hline 7 & 13,46 & 8,38 & 8,46 & 2,81 & 9,72 & 4,45 & 13,21 & 3,39 & 84,52 & 19,92 & 67,01 & 14,76 \\
\hline 8 & 5,18 & 0,91 & 3,51 & 0,64 & $\underline{4,22}$ & $\underline{0,67}$ & 11,49 & 1,91 & 103,73 & 20,89 & 83,16 & 18,13 \\
\hline 9 & 3,89 & 0,82 & 5,63 & 1,27 & $\underline{5,54}$ & $\underline{0,99}$ & 9,41 & 1,74 & 77,11 & 17,30 & 50,62 & 11,50 \\
\hline 10 & 4,47 & 1,10 & 4,28 & 0,68 & $\underline{4,67}$ & $\underline{0,89}$ & 7,00 & 1,05 & 71,12 & 16,17 & 68,42 & 14,46 \\
\hline 11 & 4,08 & 0,90 & 4,58 & 0,64 & $\underline{4,43}$ & $\underline{0,82}$ & 10,58 & 1,81 & 89,24 & 17,74 & 82,96 & 16,09 \\
\hline 12 & 4,26 & 0,78 & 4,17 & 0,90 & $\underline{4,64}$ & $\underline{0,97}$ & 8,83 & 1,36 & 78,61 & 17,80 & 69,45 & 15,22 \\
\hline \multicolumn{13}{|l|}{ Arrow } \\
\hline 1 & 9,36 & 4,33 & 6,42 & 1,91 & 6,56 & 1,87 & 12,31 & 2,04 & 75,36 & 13,20 & 61,34 & 11,16 \\
\hline 2 & 5,36 & 1,62 & 5,05 & 0,79 & 9,41 & 5,43 & 10,29 & 3,96 & 49,67 & 9,82 & 58,25 & 9,83 \\
\hline 3 & 4,81 & 0,75 & 7,66 & 2,93 & 5,60 & 0,78 & 10,65 & 1,38 & 68,15 & 11,81 & 56,36 & 10,18 \\
\hline 4 & 4,49 & 0,54 & 5,41 & 1,06 & 8,65 & 2,48 & 8,66 & 1,31 & 65,62 & 12,65 & 52,51 & 9,99 \\
\hline 5 & 6,92 & 1,89 & 7,23 & 1,96 & 6,63 & 1,20 & 11,34 & 2,53 & 65,91 & 11,29 & 67,91 & 11,31 \\
\hline 6 & 6,22 & 1,33 & 6,70 & 1,71 & 6,70 & 1,87 & 10,21 & 1,45 & 83,22 & 13,75 & 71,76 & 10,53 \\
\hline \multicolumn{13}{|l|}{ Score } \\
\hline 7 & 4,57 & 1,40 & 4,60 & 1,44 & $\underline{3,96}$ & $\underline{0,81}$ & $\underline{6,17}$ & $\underline{0,95}$ & $\underline{46,41}$ & $\underline{8,93}$ & $\underline{39,46}$ & $\underline{7,67}$ \\
\hline 8 & 4,86 & 0,55 & 4,57 & 0,47 & $\underline{5,03}$ & $\underline{0,48}$ & $\underline{8,19}$ & $\underline{0,83}$ & 62,68 & 10,12 & 59,88 & 8,64 \\
\hline 9 & 6,11 & 0,91 & 7,53 & 1,85 & 7,22 & 1,36 & $\underline{11,06}$ & $\underline{1,14}$ & $\underline{77,45}$ & $\underline{8,85}$ & $\underline{67,10}$ & $\underline{7,59}$ \\
\hline 10 & 10,14 & 4,01 & 9,14 & 1,47 & $\underline{14,37}$ & $\underline{5,31}$ & $\underline{18,29}$ & $\underline{4,27}$ & $\underline{84,11}$ & $\underline{11,98}$ & $\underline{79,19}$ & $\underline{10,63}$ \\
\hline
\end{tabular}

Fonte: Fernando Carvalheiro Reiser (2019)

Post hoc: $\mathrm{p}<0.05$ em itálico/sublinhado 
A tabela 21 mostra o índice GD-PMC nos blocos, flechas e escores em média e erro padrão. Não houve efeito neste índice no APA5 do bloco $\left(F_{11,520}=0.5 ; \mathrm{p}=0.9\right)$, flecha $\left(F_{5,520}=0.7 ; \mathrm{p}=0.6\right)$ e escore $\left(F_{3,520}<0.1 ; \mathrm{p}=0.9\right)$. Não houve efeito neste índice no APA4 do bloco $\left(F_{11,520}=0.7 ; \mathrm{p}=0.7\right)$, flecha $\left(F_{5,520}=0.6 ; \mathrm{p}=0.7\right)$ e escore $\left(F_{3,520}=0.2\right.$ $\mathrm{p}=0.9)$. Não houve efeito no APA3 do bloco $\left(F_{11,520}=0.6 ; \mathrm{p}=0.8\right)$, flecha $\left(F_{5,520}=0.2\right.$ $\mathrm{p}=0.9)$ e escore $\left(F_{3,520}=1.5 ; \mathrm{p}=0.2\right)$. Não houve efeito no APA2 do bloco $\left(F_{11,520}=0.6\right.$ $\mathrm{p}=0.8)$, flecha $\left(F_{5,520}=0.3 ; \mathrm{p}=0.9\right)$ e escore $\left(F_{3,520}=1.7 ; \mathrm{p}=0.2\right)$. Não houve efeito no APA1 do bloco $\left(F_{11,520}=0.5 ; \mathrm{p}=0.9\right)$, flecha $\left(F_{5,520}=0.8 ; \mathrm{p}=0.6\right)$, e houve efeito do escores $\left(F_{3,520}=2.9 ; \mathrm{p}=0.04\right)$, sem detecção no post hoc. Não houve efeito APC1 para bloco $\left(F_{11,520}=0.7 ; \mathrm{p}=0.71\right)$, flecha $\left(F_{5,520}=0.7 ; \mathrm{p}=0.8\right)$, e escore $\left(F_{3,520}=1.4 ; \mathrm{p}=0.24\right)$.

Tabela 21. Inibição reciproca do par GD-PMC com Média e EP

\begin{tabular}{|c|c|c|c|c|c|c|c|c|c|c|c|c|}
\hline \multirow[t]{2}{*}{ Ends } & \multicolumn{2}{|c|}{$\begin{array}{l}\text { GD-PMC } \\
\text { (APA5) }\end{array}$} & \multicolumn{2}{|c|}{$\begin{array}{c}\text { GD-PMC } \\
\text { (APA4) }\end{array}$} & \multicolumn{2}{|c|}{$\begin{array}{l}\text { GD-PMC } \\
\text { (APA3) }\end{array}$} & \multicolumn{2}{|c|}{$\begin{array}{c}\text { GD-PMC } \\
\text { (APA2) }\end{array}$} & \multicolumn{2}{|c|}{$\begin{array}{l}\text { GD-PMC } \\
\text { (APA1) }\end{array}$} & \multicolumn{2}{|c|}{$\begin{array}{c}\text { GD-PMC } \\
\text { (APC1) }\end{array}$} \\
\hline & Mean & SE & Mean & SE & Mean & SE & Mean & SE & Mean & SE & Mean & SE \\
\hline 1 & 1,43 & 0,21 & 1,50 & 0,19 & 1,45 & 0,17 & 8,44 & 1,97 & 40,57 & 9,95 & 44,81 & 10,28 \\
\hline 2 & 13,43 & 6,15 & 20,05 & 7,97 & 16,85 & 5,92 & 29,58 & 9,63 & 48,22 & 12,98 & 49,09 & 11,83 \\
\hline 3 & 14,43 & 5,94 & 5,42 & 2,22 & 11,01 & 5,28 & 19,10 & 4,78 & 60,70 & 11,30 & 85,23 & 13,91 \\
\hline 4 & 4,61 & 3,13 & 2,58 & 1,34 & 5,63 & 3,81 & 12,55 & 3,95 & 42,57 & 9,93 & 62,79 & 12,47 \\
\hline 5 & 9,34 & 5,71 & 2,74 & 0,71 & 3,04 & 0,74 & 15,37 & 3,39 & 49,82 & 10,37 & 55,92 & 12,37 \\
\hline 6 & 13,78 & 7,25 & 13,04 & 7,56 & 8,13 & 4,43 & 14,33 & 4,32 & 48,79 & 11,33 & 55,79 & 12,69 \\
\hline 7 & 5,46 & 2,42 & 7,61 & 3,10 & 9,51 & 4,31 & 19,09 & 5,37 & 38,40 & 9,17 & 43,53 & 11,18 \\
\hline 8 & 2,92 & 1,74 & 4,62 & 2,41 & 3,47 & 1,65 & 10,93 & 3,05 & 34,71 & 7,99 & 31,66 & 8,23 \\
\hline 9 & 9,92 & 5,64 & 5,67 & 2,76 & 4,57 & 2,00 & 15,63 & 4,05 & 61,47 & 12,62 & 60,01 & 13,44 \\
\hline 10 & 4,48 & 2,87 & 1,81 & 0,27 & 1,59 & 0,28 & 12,05 & 3,10 & 45,13 & 9,20 & 51,24 & 9,93 \\
\hline 11 & 2,74 & 1,05 & 4,59 & 1,88 & 3,70 & 1,63 & 12,60 & 3,57 & 41,20 & 8,54 & 47,77 & 9,24 \\
\hline 12 & 10,72 & 4,88 & 7,04 & 3,66 & 8,86 & 4,89 & 19,91 & 6,08 & 47,61 & 11,72 & 55,04 & 9,52 \\
\hline \multicolumn{13}{|l|}{ Arrow } \\
\hline 1 & 5,35 & 2,38 & 3,70 & 1,50 & 4,89 & 2,23 & 15,08 & 3,39 & 47,92 & 7,39 & 50,10 & 7,90 \\
\hline 2 & 10,23 & 4,28 & 8,26 & 3,84 & 6,04 & 1,98 & 12,32 & 2,70 & 40,13 & 6,65 & 53,46 & 8,62 \\
\hline 3 & 5,95 & 2,65 & 5,52 & 2,05 & 5,51 & 2,24 & 16,58 & 3,25 & 53,35 & 8,46 & 58,90 & 8,32 \\
\hline 4 & 12,46 & 4,07 & 11,04 & 4,04 & 8,10 & 2,93 & 16,20 & 3,82 & 43,76 & 6,71 & 59,38 & 8,21 \\
\hline 5 & 7,58 & 3,01 & 5,68 & 1,77 & 6,22 & 2,39 & 16,02 & 3,79 & 41,94 & 7,56 & 46,53 & 7,87 \\
\hline 6 & 5,06 & 2,00 & 4,14 & 1,39 & 8,14 & 3,13 & 18,58 & 3,47 & 52,48 & 7,70 & 53,07 & 7,57 \\
\hline \multicolumn{13}{|l|}{ Score } \\
\hline 7 & 10,39 & 3,60 & 5,82 & 1,72 & 4,84 & 1,68 & 14,45 & 3,07 & 42,66 & 7,04 & 42,26 & 6,69 \\
\hline 8 & 7,40 & 2,71 & 7,99 & 3,28 & 5,72 & 1,90 & 16,93 & 2,97 & 54,28 & 6,41 & 59,65 & 7,12 \\
\hline 9 & 7,51 & 2,00 & 4,65 & 1,13 & 6,71 & 1,94 & 12,04 & 1,95 & 38,16 & 4,57 & 53,77 & 5,52 \\
\hline 10 & 5,65 & 1,77 & 7,95 & 2,22 & 9,08 & 2,69 & 22,56 & 3,66 & 55,68 & 6,88 & 58,17 & 7,32 \\
\hline
\end{tabular}

Fonte: Fernando Carvalheiro Reiser (2019)

Na tabela 22 está o índice TS-SAD (blocos, flechas e escores). No APA5, houve efeito do bloco $\left(F_{11,520}=2.3 ; \mathrm{p}<0.01\right)$; com maior ativação no 12 do que em 1 e 2 $(\mathrm{p}=0.05)$, não houve da flecha $\left(F_{5,520}=1.9 \mathrm{p}=0.1\right)$ e escore $\left(F_{3,520}=2.0 \mathrm{p}=0.1\right)$. Não houve efeito no APA4 do bloco $\left(F_{11,520}=1.2 \mathrm{p}=0.3\right)$, flecha $\left(F_{5,520}=1.2 \mathrm{p}=0.3\right)$ e escore $\left(F_{3,520}=0.7 \mathrm{p}=0.6\right)$. Não houve efeito no APA3 do bloco $\left(F_{11,520}=0.4 ; \mathrm{p}=0.9\right)$, flecha 
$\left(F_{5,520}=0.5 \mathrm{p}=0.7\right)$, e escore $\left(F_{3,520}=0.2 ; \mathrm{p}=0.9\right)$. Não houve efeito no APA2 do bloco $\left(F_{11,520}=1.3 \mathrm{p}=0.22\right)$, houve efeito da flecha $\left(F_{5,520}=2.2 ; \mathrm{p}=0.05\right)$; maior na flecha 5 comparada a $2(\mathrm{p}=0.03)$. Não houve efeito do escore $(F 3,520=1.4 ; \mathrm{p}=0.2)$. No APA1 houve efeito do bloco $\left(F_{11,520}=2.5 ; \mathrm{p}<0.01\right)$; maior no 4 do que 9 (p<0.05). Não houve efeito da flecha $\left(F_{5,520}=0.1 \mathrm{p}=0.9\right)$ e escore $\left(F_{3,520}=2.2 \mathrm{p}=0.1\right)$. Não houve efeito no APC1 do bloco $\left(F_{11,520}=1.4 \mathrm{p}=0.2\right)$, flecha $\left(F_{5,520}=0.9 \mathrm{p}=0.4\right)$ e escore $\left(F_{3,520}=1.6 \mathrm{p}=0.2\right)$.

Tabela 22. Inibição reciproca do par TS-SAD com Média e EP

\begin{tabular}{|c|c|c|c|c|c|c|c|c|c|c|c|c|}
\hline \multirow[t]{2}{*}{ Ends } & \multicolumn{2}{|c|}{$\begin{array}{l}\text { TS-SAD } \\
\text { (APA5) }\end{array}$} & \multicolumn{2}{|c|}{$\begin{array}{l}\text { TS-SAD } \\
\text { (APA4) }\end{array}$} & \multicolumn{2}{|c|}{$\begin{array}{l}\text { TS-SAD } \\
\text { (APA3) }\end{array}$} & \multicolumn{2}{|c|}{$\begin{array}{l}\text { TS-SAD } \\
\text { (APA2) }\end{array}$} & \multicolumn{2}{|c|}{$\begin{array}{l}\text { TS-SAD } \\
\text { (APA1) }\end{array}$} & \multicolumn{2}{|c|}{$\begin{array}{l}\text { TS-SAD } \\
\text { (APC1) }\end{array}$} \\
\hline & Mean & SE & Mean & SE & Mean & SE & Mean & SE & Mean & SE & Mean & SE \\
\hline 1 & $\underline{3,65}$ & $\underline{0,57}$ & 3,59 & 0,55 & 4,31 & 0,96 & 5,81 & 1,08 & 57,72 & 15,79 & 72,54 & 18,10 \\
\hline 2 & $\underline{3,63}$ & $\underline{0,51}$ & 3,16 & 0,50 & 4,70 & 1,04 & 11,36 & 5,18 & 30,82 & 8,63 & 50,62 & 16,36 \\
\hline 3 & 5,73 & 1,27 & 5,95 & 1,32 & 6,39 & 1,38 & 10,75 & 2,73 & 39,91 & 9,76 & 50,72 & 11,09 \\
\hline 4 & 6,48 & 1,77 & 6,20 & 1,39 & 4,51 & 1,09 & 15,72 & 5,74 & $\underline{80,68}$ & $\underline{17,02}$ & 92,71 & 22,37 \\
\hline 5 & 8,06 & 2,62 & 7,03 & 1,53 & 5,65 & 0,99 & 9,48 & 1,50 & 74,36 & 16,91 & 77,26 & 18,36 \\
\hline 6 & 11,17 & 3,97 & 7,55 & 1,59 & 7,49 & 1,86 & 9,93 & 1,79 & 73,30 & 15,57 & 67,42 & 14,83 \\
\hline 7 & 9,07 & 3,61 & 11,36 & 6,95 & 7,03 & 2,86 & 5,90 & 0,89 & 29,02 & 8,33 & 40,39 & 12,80 \\
\hline 8 & 6,43 & 1,24 & 4,05 & 0,68 & 5,54 & 1,02 & 7,66 & 2,03 & 32,06 & 6,81 & 37,29 & 9,49 \\
\hline 9 & 5,13 & 0,95 & 6,60 & 1,26 & 13,75 & 5,92 & 10,70 & 3,33 & 21,09 & 7,45 & 43,57 & 13,55 \\
\hline 10 & 5,75 & 1,01 & 5,04 & 0,97 & 6,06 & 0,99 & 8,12 & 1,66 & 37,66 & 9,02 & 37,64 & 10,51 \\
\hline 11 & 5,24 & 1,17 & 5,73 & 0,91 & 10,95 & 5,86 & 9,18 & 2,58 & 44,18 & 11,72 & 51,13 & 13,95 \\
\hline 12 & $\underline{14,48}$ & $\underline{5,34}$ & 9,23 & 2,95 & 12,16 & 6,61 & 14,33 & 4,40 & 44,79 & 9,60 & 49,62 & 13,07 \\
\hline \multicolumn{13}{|l|}{ Arrow } \\
\hline 1 & 5,59 & 1,17 & 5,01 & 0,59 & 8,50 & 2,99 & 9,62 & 1,83 & 50,74 & 8,92 & 62,34 & 12,76 \\
\hline 2 & 6,50 & 2,02 & 5,23 & 0,81 & $\underline{3,86}$ & $\underline{0,40}$ & 5,25 & 0,79 & 46,48 & 8,80 & 54,49 & 10,47 \\
\hline 3 & 5,80 & 0,82 & 4,44 & 0,62 & 5,34 & 0,59 & 7,74 & 0,97 & 47,63 & 8,27 & 60,65 & 10,62 \\
\hline 4 & 10,03 & 2,77 & 6,88 & 1,52 & 9,14 & 3,37 & 9,33 & 2,02 & 49,41 & 8,18 & 54,21 & 9,96 \\
\hline 5 & 8,11 & 2,01 & 9,89 & 3,56 & $\underline{10,30}$ & $\underline{3,31}$ & 14,57 & 3,34 & 49,85 & 9,91 & 40,97 & 9,21 \\
\hline 6 & 6,38 & 0,95 & 6,30 & 1,02 & 7,14 & 1,16 & 12,97 & 3,04 & 38,68 & 7,19 & 62,80 & 10,41 \\
\hline \multicolumn{13}{|l|}{ Score } \\
\hline 7 & 10,01 & 2,50 & 7,82 & 2,68 & 6,19 & 1,15 & 7,55 & 0,87 & 47,77 & 8,87 & 62,48 & 11,17 \\
\hline 8 & 6,69 & 0,92 & 6,63 & 1,07 & 7,99 & 2,20 & 9,10 & 1,57 & 38,89 & 5,85 & 47,89 & 7,86 \\
\hline 9 & 5,37 & 0,58 & 5,23 & 0,57 & 7,67 & 2,11 & 9,85 & 1,67 & 59,76 & 6,70 & 61,51 & 7,53 \\
\hline 10 & 7,15 & 1,87 & 5,91 & 0,80 & 7,42 & 1,03 & 13,97 & 2,99 & 35,24 & 5,39 & 49,27 & 8,03 \\
\hline
\end{tabular}

Fonte: Fernando Carvalheiro Reiser (2019)

Post hoc: $\mathrm{p}<0.05$ em itálico/sublinhado

A Tabela 23 mostra o índice RA-ML nos blocos, flechas e escores em média e erro padrão. Não houve efeito no APA5 do bloco $\left(F_{11,520}=1.1 ; \mathrm{p}=0.3\right)$, flecha $\left(F_{5,520}=0.6\right.$; $\mathrm{p}=0.7)$ e escore $\left(F_{3,520}=0.8 ; \mathrm{p}=0.5\right)$. Não houve efeito no APA4 do bloco $\left(F_{11,520}=1.0\right.$; $\mathrm{p}=0.5)$, flecha $\left(F_{5,520}=0.3 ; \mathrm{p}=0.9\right)$ e escore $\left(F_{3,520}=1.5 ; \mathrm{p}=0.2\right)$. Não houve efeito no APA3 para bloco $\left(F_{11,520}=1.0 ; \mathrm{p}=0.5\right)$, flecha $\left(F_{5,520}=0.3 ; \mathrm{p}=0.9\right)$, e escore $\left(F_{3,520}=1.0\right.$; $\mathrm{p}=0.4)$. Não houve efeito no APA2 para bloco $\left(F_{11,520}=1.0 ; \mathrm{p}=0.6\right)$, flecha $\left(F_{5,520}=0.1\right.$; 
$\mathrm{p}=0.9)$ e escore $\left(F_{3,520}=1.6 ; \mathrm{p}=0.2\right)$. Não houve efeito no APA1 para bloco $\left(F_{11,520}=0.7\right.$;

$\mathrm{p}=0.7)$, flechas $\left(F_{5,520}=0.7 ; \mathrm{p}=0.6\right)$ e escore $\left(F_{3,520}=0.9 ; \mathrm{p}=0.42\right)$. Não houve efeito no

APC1 bloco $\left(F_{11,520}=1.5 ; \mathrm{p}=0.1\right)$; flecha $\left(F_{5,520}=1.5 ; \mathrm{p}=0.2\right)$ e escore $\left(F_{3,520}=1.6 ; \mathrm{p}=0.2\right)$.

Tabela 23. Inibição reciproca do par RA-ML com Média e EP

\begin{tabular}{|c|c|c|c|c|c|c|c|c|c|c|c|c|}
\hline \multirow[t]{2}{*}{ Ends } & \multicolumn{2}{|c|}{$\begin{array}{l}\text { RA-ML } \\
\text { (APA5) }\end{array}$} & \multicolumn{2}{|c|}{$\begin{array}{l}\text { RA-ML } \\
\text { (APA4) }\end{array}$} & \multicolumn{2}{|c|}{$\begin{array}{l}\text { RA-ML } \\
\text { (APA3) }\end{array}$} & \multicolumn{2}{|c|}{$\begin{array}{l}\text { RA-ML } \\
\text { (APA2) }\end{array}$} & \multicolumn{2}{|c|}{$\begin{array}{l}\text { RA-ML } \\
\text { (APA1) }\end{array}$} & \multicolumn{2}{|c|}{$\begin{array}{l}\text { RA-ML } \\
\text { (APC1) }\end{array}$} \\
\hline & Mean & SE & Mean & SE & Mean & $\mathrm{SE}$ & Mean & SE & Mean & SE & Mean & $\mathrm{SE}$ \\
\hline 1 & 6,32 & 4,29 & 12,97 & 7,77 & 13,49 & 8,17 & 13,72 & 8,13 & 23,45 & 9,01 & 35,96 & 10,18 \\
\hline 2 & 1,30 & 0,40 & 1,05 & 0,26 & 1,43 & 0,35 & 2,19 & 0,45 & 22,75 & 6,73 & 47,11 & 15,10 \\
\hline 3 & 3,02 & 0,76 & 8,42 & 6,57 & 9,57 & 7,05 & 9,65 & 6,99 & 21,93 & 8,71 & 35,27 & 13,77 \\
\hline 4 & 2,96 & 1,04 & 2,30 & 0,57 & 10,74 & 4,09 & 6,52 & 2,06 & 13,08 & 5,96 & 20,81 & 8,99 \\
\hline 5 & 8,33 & 3,51 & 10,66 & 4,29 & 16,64 & 5,03 & 15,35 & 4,93 & 20,04 & 5,34 & 32,71 & 9,47 \\
\hline 6 & 4,04 & 1,48 & 4,13 & 1,60 & 3,91 & 1,26 & 6,04 & 1,86 & 9,81 & 3,01 & 13,61 & 4,13 \\
\hline 7 & 2,46 & 0,61 & 2,86 & 0,61 & 2,87 & 0,55 & 4,85 & 2,58 & 19,35 & 6,89 & 40,20 & 11,77 \\
\hline 8 & 11,12 & 5,69 & 11,34 & 5,64 & 11,66 & 7,00 & 7,42 & 3,77 & 20,61 & 8,92 & 16,98 & 6,25 \\
\hline 9 & 13,93 & 9,65 & 12,77 & 7,71 & 19,36 & 8,58 & 17,21 & 7,68 & 32,60 & 10,09 & 48,78 & 14,43 \\
\hline 10 & 3,06 & 1,51 & 5,61 & 3,71 & 5,19 & 2,16 & 8,73 & 5,02 & 21,89 & 7,55 & 34,83 & 11,73 \\
\hline 11 & 13,45 & 6,47 & 13,14 & 7,27 & 15,36 & 8,46 & 24,95 & 11,35 & 20,24 & 8,30 & 23,09 & 5,70 \\
\hline 12 & 1,22 & 0,37 & 1,29 & 0,31 & 0,95 & 0,23 & 1,73 & 0,43 & 5,89 & 2,74 & 14,25 & 6,00 \\
\hline \multicolumn{13}{|l|}{ Arrow } \\
\hline 1 & 4,04 & 2,20 & 7,18 & 3,78 & 9,68 & 4,32 & 9,13 & 4,40 & 18,12 & 5,03 & 34,01 & 8,20 \\
\hline 2 & 9,73 & 5,05 & 7,24 & 3,74 & 11,23 & 4,04 & 11,46 & 3,86 & 18,70 & 4,76 & 25,31 & 5,88 \\
\hline 3 & 4,50 & 1,81 & 8,82 & 4,08 & 10,33 & 4,20 & 9,83 & 3,95 & 25,00 & 6,32 & 27,03 & 6,41 \\
\hline 4 & 7,34 & 3,37 & 5,37 & 2,42 & 8,04 & 3,08 & 9,45 & 3,88 & 22,36 & 6,14 & 42,58 & 10,30 \\
\hline 5 & 3,59 & 1,43 & 4,47 & 1,93 & 3,86 & 1,28 & 6,64 & 3,01 & 14,96 & 3,83 & 26,46 & 6,58 \\
\hline 6 & 6,40 & 2,11 & 10,20 & 4,08 & 12,44 & 5,17 & 12,66 & 4,74 & 16,68 & 4,39 & 26,40 & 5,94 \\
\hline \multicolumn{13}{|l|}{ Score } \\
\hline 7 & 5,41 & 2,12 & 6,07 & 2,20 & 6,13 & 2,27 & 4,38 & 1,66 & 12,96 & 3,20 & 24,81 & 5,88 \\
\hline 8 & 4,57 & 1,57 & 6,49 & 2,70 & 7,62 & 3,10 & 8,09 & 2,76 & 21,10 & 4,41 & 25,93 & 5,45 \\
\hline 9 & 5,40 & 1,65 & 5,04 & 1,40 & 10,38 & 2,33 & 12,05 & 2,96 & 21,24 & 3,49 & 40,24 & 6,16 \\
\hline 10 & 9,42 & 4,64 & 13,48 & 5,43 & 13,30 & 5,40 & 14,94 & 5,47 & 20,87 & 6,03 & 25,09 & 5,77 \\
\hline
\end{tabular}

Fonte: Fernando Carvalheiro Reiser (2019)

4.3.2 Distal-Proximal (Punho e cotovelo)

O índice agonista FSD-BBL está na tabela 24. Não houve efeito no APA5 do bloco $\left(F_{11,520}=0.5 ; \mathrm{p}=0.9\right)$, flecha $\left(F_{5,520}=0.3 ; \mathrm{p}=0.9\right)$ e escore $\left(F_{3,520}=2.4 ; \mathrm{p}=0.1\right)$. Não houve efeito no APA4 do bloco $\left(F_{11,520}=1.7 ; \mathrm{p}=0.9\right)$, flecha $\left(F_{5,520}=0.3 ; \mathrm{p}=0.9\right)$ e escore $\left(F_{3,520}=2.3 ; \mathrm{p}=0.1\right)$. Houve efeito nesse índice no APA3 no bloco $\left(F_{11,520}=8.3 ; \mathrm{p}<0.01\right)$, maior no 5 comparado ao 4 ( $\mathrm{p}=0.02)$, houve efeito na flecha $\left(F_{5,520}=5.8 ; \mathrm{p}<0.01\right)$; mas o post hoc falhou na detecção, houve efeito no escore $\left(F_{3,520}=12.0 ; \mathrm{p}<0.01\right)$; maior no 10 em relação ao 7 e $9(\mathrm{p}<0.01)$. Houve efeito nesse índice para APA2 no bloco $\left(F_{11,520}=2.4 ;\right.$ p $\left.<0.01\right)$, maior no 2 comparado ao 8,10 e 12 , não houve efeito nas flechas $\left(F_{5,520}=0.6 ; \mathrm{p}=0.7\right)$ e escore $\left(F_{3,520}=1.5 ; \mathrm{p}=0.2\right)$. Não houve efeito no APA1 do bloco $\left(F_{11,520}=1.6 ; \mathrm{p}=0.1\right)$, flecha $\left(F_{5,520}=0.4 ; \mathrm{p}=0.8\right)$, e escore $\left(F_{3,520}=0.4 ; \mathrm{p}=0.7\right)$. Não houve 
efeito no APC1 do bloco $\left(F_{11,520}=1.7 ; \mathrm{p}=0.1\right)$, flechas $\left(F_{5,520}=0.4 ; \mathrm{p}=0.8\right)$, mas houve no escore $\left(F_{3,520}=3.9 ; \mathrm{p}=0.01\right)$; maior no escore 10 comparado ao 7 e $8(\mathrm{p}<0.01)$.

Tabela 24. Inibição reciproca do par FSD-BBL com Média e EP

\begin{tabular}{|c|c|c|c|c|c|c|c|c|c|c|c|c|}
\hline \multirow[t]{2}{*}{ Ends } & \multicolumn{2}{|c|}{$\begin{array}{c}\text { FSD-BBL } \\
\text { (APA5) }\end{array}$} & \multicolumn{2}{|c|}{$\begin{array}{l}\text { FSD-BBL } \\
\text { (APA4) }\end{array}$} & \multicolumn{2}{|c|}{$\begin{array}{c}\text { FSD-BBL } \\
\text { (APA3) }\end{array}$} & \multicolumn{2}{|c|}{$\begin{array}{l}\text { FSD-BBL } \\
\text { (APA2) }\end{array}$} & \multicolumn{2}{|c|}{$\begin{array}{l}\text { FSD-BBL } \\
\text { (APA1) }\end{array}$} & \multicolumn{2}{|c|}{$\begin{array}{l}\text { FSD-BBL } \\
\text { (APC1) }\end{array}$} \\
\hline & Mean & SE & Mean & SE & Mean & $\mathrm{SE}$ & Mean & $\mathrm{SE}$ & Mean & SE & Mean & SE \\
\hline 1 & 2,39 & 0,53 & 3,19 & 0,61 & 3,43 & 0,67 & 11,32 & 2,21 & 54,74 & 12,54 & 42,27 & 12,19 \\
\hline 2 & 3,50 & 0,96 & 4,35 & 1,20 & 4,37 & 1,27 & $\underline{13,74}$ & $\underline{2,91}$ & 52,92 & 12,53 & 51,21 & 15,01 \\
\hline 3 & 3,35 & 0,56 & 3,95 & 0,74 & 3,58 & 0,58 & 9,68 & 2,63 & 69,37 & 25,44 & 83,48 & 21,34 \\
\hline 4 & 2,52 & 0,41 & 2,77 & 0,64 & $\underline{1,85}$ & $\underline{0,27}$ & 7,42 & 1,24 & 57,14 & 15,07 & 37,58 & 11,65 \\
\hline 5 & 2,77 & 0,44 & 3,27 & 0,58 & $\underline{4,89}$ & $\underline{2,15}$ & 5,94 & 1,55 & 54,10 & 18,23 & 51,36 & 15,17 \\
\hline 6 & 2,95 & 0,62 & 3,33 & 0,55 & 2,91 & 0,51 & 6,29 & 1,31 & 23,35 & 8,49 & 30,91 & 10,67 \\
\hline 7 & 2,54 & 0,32 & 2,17 & 0,35 & 2,80 & 0,41 & 8,03 & 1,98 & 39,61 & 11,65 & 41,67 & 11,78 \\
\hline 8 & 3,07 & 0,67 & 3,19 & 0,51 & 3,02 & 0,48 & $\underline{5,32}$ & $\underline{1,25}$ & 20,40 & 6,79 & 31,06 & 10,56 \\
\hline 9 & 2,50 & 0,38 & 3,31 & 0,57 & 3,34 & 0,70 & 7,52 & 1,53 & 35,44 & 8,83 & 32,12 & 7,82 \\
\hline 10 & 2,56 & 0,46 & 2,50 & 0,33 & 2,66 & 0,37 & $\underline{4,23}$ & $\underline{0,76}$ & 20,80 & 6,09 & 38,79 & 10,51 \\
\hline 11 & 2,42 & 0,46 & 2,18 & 0,32 & 2,40 & 0,37 & 5,67 & 1,41 & 33,67 & 10,22 & 44,70 & 11,57 \\
\hline 12 & 2,73 & 0,41 & 2,31 & 0,40 & 2,27 & 0,33 & $\underline{4,32}$ & $\underline{0,92}$ & 20,46 & 5,81 & 31,36 & 9,82 \\
\hline \multicolumn{13}{|l|}{ Arrow } \\
\hline 1 & 2,52 & 0,35 & 2,53 & 0,36 & 2,86 & 0,33 & 8,02 & 1,31 & 34,58 & 7,60 & 37,88 & 8,41 \\
\hline 2 & 2,93 & 0,52 & 3,41 & 0,64 & 3,22 & 0,66 & 6,62 & 1,08 & 39,56 & 9,39 & 45,76 & 9,22 \\
\hline 3 & 2,60 & 0,33 & 2,83 & 0,35 & 3,18 & 0,42 & 8,56 & 1,43 & 46,59 & 11,00 & 36,97 & 8,29 \\
\hline 4 & 3,07 & 0,43 & 3,15 & 0,42 & 3,83 & 1,12 & 7,05 & 1,17 & 43,85 & 8,54 & 33,41 & 7,31 \\
\hline 5 & 2,66 & 0,33 & 3,32 & 0,41 & 2,77 & 0,32 & 8,32 & 1,51 & 45,66 & 11,33 & 50,38 & 9,77 \\
\hline 6 & 2,87 & 0,31 & 3,02 & 0,36 & 2,91 & 0,32 & 6,17 & 0,99 & 30,75 & 6,75 & 53,86 & 10,94 \\
\hline \multicolumn{13}{|l|}{ Score } \\
\hline 7 & 2,46 & 0,25 & 2,71 & 0,26 & $\underline{2,51}$ & $\underline{0,26}$ & 6,54 & 0,99 & 37,26 & 8,31 & 27,31 & $\underline{5,55}$ \\
\hline 8 & 3,14 & 0,42 & 3,40 & 0,47 & 3,11 & 0,46 & 6,69 & 0,87 & 34,02 & 5,70 & 33,01 & $\underline{6,26}$ \\
\hline 9 & 2,37 & 0,21 & 2,76 & 0,23 & $\underline{2,84}$ & $\underline{0,26}$ & 8,01 & 0,99 & 44,23 & 7,93 & 47,61 & 6,87 \\
\hline 10 & 3,36 & 0,37 & 3,44 & 0,46 & $\underline{4,39}$ & $\underline{1,01}$ & 8,63 & 1,27 & 44,94 & 7,01 & 67,62 & 10,87 \\
\hline
\end{tabular}

O índice agonista-antagonista FSD-TBL está na tabela 25. Não houve efeito no APA5 do bloco $\left(F_{11,520}=1.1 ; \mathrm{p}=0.4\right)$, flecha $(F 5,520=0.5 ; \mathrm{p}=0.8)$ e escore $\left(F_{3,520}=0.1\right.$; $\mathrm{p}=0.4)$. Não houve efeito no APA4 do bloco $\left(F_{11,520}=1.13 ; \mathrm{p}=0.3\right)$, flecha $\left(F_{5,520}=0.2\right.$; $\mathrm{p}=0.9)$, e escore $\left(F_{3,520}=1.0 ; \mathrm{p}=0.4\right)$. Houve efeito no APA3 no bloco $\left(F_{11,520}=2.0\right.$; $\mathrm{p}=0.03$ ), com maior modulação no 4 em relação $1,6,8,10-12(\mathrm{p}<0.05)$, não houve efeito na flecha $\left(F_{5,520}=1.3 ; \mathrm{p}=0.3\right)$. Houve efeito do escore $\left(F_{3,520}=3.6 ; \mathrm{p}<0.02\right)$ maior no 10 em relação ao 8 e 7 (p<0.01). Não houve efeito no APA2 do bloco $\left(F_{11,520}=1.2 ; \mathrm{p}=0.32\right)$ e flechas $\left(F_{5,520}=0.9 ; \mathrm{p}=0.5\right)$. Houve efeito do escore $\left(F_{3,520}=5.4 ; \mathrm{p}<0.01\right)$ maior no 10 comparado ao 7 e $8(\mathrm{p}<0.01)$. Não houve efeito no APA1 do bloco $\left(F_{11,520}=1.1 ; \mathrm{p}=0.4\right)$ e flecha $\left(F_{5,520}=1.1 ; \mathrm{p}=0.4\right)$. Houve efeito do escore $\left(F_{3,520}=3.9 ; \mathrm{p}=0.01\right)$ maior no 9 comparado ao escore 7 e $8(\mathrm{p}<0.01)$. Não houve efeito no APC1 do bloco $\left(F_{11,520}=1.0\right.$; 
$\mathrm{p}=0.5)$ e flecha $\left(F_{5,520}=0.8 ; \mathrm{p}=0.5\right)$. Houve efeito no escore $\left(F_{3,520}=6.4 ; \mathrm{p}<0.01\right)$; maior nos de 10 e 9 em comparação com 7 ( $\mathrm{p}<0.01)$.

Tabela 25. Inibição reciproca do par FSD-TBL com Média e EP

\begin{tabular}{|c|c|c|c|c|c|c|c|c|c|c|c|c|}
\hline \multirow[t]{2}{*}{ Ends } & \multicolumn{2}{|c|}{$\begin{array}{c}\text { FSD-TBL } \\
\text { (APA5) }\end{array}$} & \multicolumn{2}{|c|}{$\begin{array}{c}\text { FSD-TBL } \\
\text { (APA4) }\end{array}$} & \multicolumn{2}{|c|}{$\begin{array}{l}\text { FSD-TBL } \\
\text { (APA3) }\end{array}$} & \multicolumn{2}{|c|}{$\begin{array}{c}\text { FSD-TBL } \\
\text { (APA2) }\end{array}$} & \multicolumn{2}{|c|}{$\begin{array}{c}\text { FSD-TBL } \\
\text { (APA1) }\end{array}$} & \multicolumn{2}{|c|}{$\begin{array}{l}\text { FSD-TBL } \\
\text { (APC1) }\end{array}$} \\
\hline & Mean & SE & Mean & $\mathrm{SE}$ & Mean & SE & Mean & SE & Mean & SE & Mean & SE \\
\hline 1 & 4,79 & 0,70 & 4,92 & 0,60 & 4,99 & $\underline{0,66}$ & 7,00 & 1,14 & 35,23 & 10,71 & 39,85 & 11,47 \\
\hline 2 & 6,02 & 1,09 & 6,38 & 1,24 & 7,09 & 1,44 & 13,18 & 4,60 & 42,29 & 12,22 & 52,73 & 12,86 \\
\hline 3 & 7,20 & 1,30 & 7,11 & 1,16 & 7,52 & 1,49 & 8,30 & 2,14 & 61,70 & 20,94 & 77,73 & 16,70 \\
\hline 4 & 14,56 & 5,12 & 18,55 & 7,46 & 22,48 & 11,49 & 16,85 & 7,55 & 66,74 & 13,99 & 66,82 & 15,16 \\
\hline 5 & 7,52 & 2,26 & 9,09 & 3,29 & 8,97 & 3,67 & 6,82 & 1,88 & 35,11 & 9,65 & 57,42 & 14,06 \\
\hline 6 & 6,47 & 1,31 & 5,65 & 0,89 & $\underline{5,54}$ & $\underline{0,81}$ & 6,88 & 1,42 & 50,21 & 14,79 & 66,36 & 15,72 \\
\hline 7 & 14,05 & 8,38 & 8,94 & 2,82 & 11,31 & 4,57 & 10,59 & 2,99 & 62,56 & 17,72 & 70,30 & 14,53 \\
\hline 8 & 5,82 & 0,87 & 5,07 & 0,80 & $\underline{4,69}$ & $\underline{0,70}$ & 10,98 & 1,88 & 100,84 & 21,57 & 95,76 & 18,94 \\
\hline 9 & 4,68 & 0,93 & 6,58 & 1,32 & 7,18 & 1,11 & 8,23 & 1,55 & 56,44 & 16,29 & 43,33 & 10,83 \\
\hline 10 & 5,32 & 1,12 & 4,69 & 0,72 & $\underline{5,85}$ & $\underline{1,10}$ & 7,42 & 1,26 & 64,95 & 15,39 & 69,85 & 13,32 \\
\hline 11 & 4,80 & 0,98 & 4,90 & 0,74 & 4,82 & $\underline{0,90}$ & 8,02 & 1,42 & 65,93 & 14,35 & 71,82 & 13,31 \\
\hline 12 & 4,73 & 0,85 & 4,97 & 1,07 & $\underline{4,42}$ & $\underline{1,04}$ & 8,17 & 1,38 & 79,31 & 18,41 & 84,39 & 16,11 \\
\hline \multicolumn{13}{|l|}{ Arrow } \\
\hline 1 & 9,85 & 4,33 & 7,33 & 1,91 & 8,22 & 1,96 & 10,65 & 1,86 & 66,88 & 13,16 & 71,29 & 11,68 \\
\hline 2 & 6,44 & 1,67 & 6,16 & 0,96 & 10,27 & 5,46 & 10,75 & 3,76 & 51,47 & 10,33 & 65,23 & 9,93 \\
\hline 3 & 6,05 & 0,84 & 8,28 & 2,95 & 5,89 & 0,83 & 8,00 & 1,20 & 53,81 & 11,02 & 59,85 & 10,07 \\
\hline 4 & 5,50 & 0,63 & 5,89 & 1,06 & 8,29 & 2,30 & 8,14 & 1,00 & 52,50 & 9,94 & 53,03 & 9,10 \\
\hline 5 & 8,05 & 1,91 & 8,23 & 1,95 & 7,42 & 1,24 & 10,27 & 2,46 & 65,79 & 11,58 & 70,00 & 10,14 \\
\hline 6 & 7,08 & 1,31 & 7,53 & 1,72 & 7,35 & 1,90 & 8,42 & 1,23 & 70,21 & 11,45 & 78,79 & 10,90 \\
\hline \multicolumn{13}{|l|}{ Score } \\
\hline 7 & 5,47 & 1,41 & 5,62 & 1,45 & $\underline{4,62}$ & $\underline{0,85}$ & $\underline{5,96}$ & $\underline{0,80}$ & 45,05 & 9,21 & 43,60 & $\underline{7,70}$ \\
\hline 8 & 6,08 & 0,64 & 5,43 & 0,54 & $\underline{5,81}$ & $\underline{0,61}$ & $\underline{6,81}$ & $\underline{0,69}$ & $\underline{47,18}$ & $\underline{7,82}$ & 60,53 & 7,75 \\
\hline 9 & 7,00 & 0,93 & 8,48 & 1,86 & 7,74 & 1,38 & 9,95 & 1,06 & 77,75 & 9,21 & 74,77 & $\underline{7,67}$ \\
\hline 10 & 10,98 & 4,01 & 9,45 & 1,47 & $\underline{15,05}$ & $\underline{5,27}$ & $\underline{15,97}$ & $\underline{4,04}$ & 64,48 & 9,80 & $\underline{86,53}$ & $\underline{10,86}$ \\
\hline
\end{tabular}

Fonte: Fernando Carvalheiro Reiser (2019)

Post hoc: $\mathrm{p}<0.05$ em itálico/sublinhado

No índice ED-BBL (tabela 26), não houve efeito no APA5 do bloco $\left(F_{11,520}=0.8\right.$; $\mathrm{p}=0.6)$, flecha $\left(F_{5,520}=0.3 ; \mathrm{p}=0.9\right)$ e escore $\left(F_{3,520}=1.2 ; \mathrm{p}=0.3\right)$. No APA4, neste índice não houve efeito do bloco $\left(F_{11,520}=1.5 ; \mathrm{p}=0.2\right)$ e flecha $\left(F_{5,520}=0.7 ; \mathrm{p}=0.6\right)$ mas houve do escore $\left(F_{3,520}=3.6 ; \mathrm{p}=0.01\right)$ maior no 10 comparado ao $7(\mathrm{p}=0.05)$. No APA3 houve efeito no bloco $\left(F_{11,520}=13.5 ; \mathrm{p}<0.01\right)$; maior no 5 em comparação ao $2,6-12$, houve efeito da flecha $\left(F_{5,520}=9.7 ; \mathrm{p}<0.01\right)$, sem detecção no post hoc, houve efeito nos escores $\left(F_{3,520}=17.3 ; \mathrm{p}<0.01\right)$ maior no 10 em relação ao 9,8 e 7 ( $\left.\mathrm{p}<0.01\right)$. Não houve efeito no APA2 para bloco $\left(F_{11,520}=1.5 ; \mathrm{p}=0.1\right)$, e flecha $\left(F_{5,520}=1.7 ; \mathrm{p}=0.1\right)$, contudo nos escores houve $\left(F_{3,520}=3.1 ; \mathrm{p}=0.02\right)$ no 10 maior que 8 e $7(\mathrm{p}=0.05)$. No APA1, não houve efeito do bloco $\left(F_{11,520}=1.1 ; \mathrm{p}=0.4\right)$, flechas $\left(F_{5,520}=0.9 ; \mathrm{p}=0.5\right)$ e escore $\left(F_{3,520}=2.0 ; \mathrm{p}=0.1\right)$. Não houve efeito no APC1 do bloco $\left(F_{11,520}=1.6 ; \mathrm{p}=0.11\right)$, flecha $\left(F_{5,520}=1.0 ; \mathrm{p}=0.4\right)$, e escores $\left(F_{3,520}=1.3 ; \mathrm{p}=0.3\right)$. 
Tabela 26. Inibição reciproca do par ED-BBL com Média e EP

\begin{tabular}{|c|c|c|c|c|c|c|c|c|c|c|c|c|}
\hline \multirow[t]{2}{*}{ Ends } & \multicolumn{2}{|c|}{$\begin{array}{l}\text { ED-BBL } \\
\text { (APA5) }\end{array}$} & \multicolumn{2}{|c|}{$\begin{array}{c}\text { ED-BBL } \\
\text { (APA4) }\end{array}$} & \multicolumn{2}{|c|}{$\begin{array}{c}\text { ED-BBL } \\
\text { (APA3) }\end{array}$} & \multicolumn{2}{|c|}{$\begin{array}{c}\text { ED-BBL } \\
\text { (APA2) }\end{array}$} & \multicolumn{2}{|c|}{$\begin{array}{l}\text { ED-BBL } \\
\text { (APA1) }\end{array}$} & \multicolumn{2}{|c|}{$\begin{array}{c}\text { ED-BBL } \\
\text { (APC1) }\end{array}$} \\
\hline & Mean & SE & Mean & SE & Mean & SE & Mean & SE & Mean & SE & Mean & SE \\
\hline 1 & 2,24 & 0,36 & 3,08 & 0,84 & 3,34 & 0,76 & 10,02 & 1,90 & 33,09 & 8,64 & 20,61 & 7,26 \\
\hline 2 & 2,57 & 0,42 & 2,92 & 0,40 & 2,49 & $\underline{0,37}$ & 10,76 & 2,32 & 45,90 & 11,85 & 18,79 & 4,86 \\
\hline 3 & 3,04 & 0,49 & 4,60 & 0,98 & 4,06 & 0,75 & 17,39 & 2,75 & 64,43 & 12,00 & 17,27 & 3,18 \\
\hline 4 & 2,97 & 0,55 & 2,91 & 0,36 & 3,45 & 0,61 & 6,73 & 1,18 & 40,35 & 11,21 & 9,09 & 1,75 \\
\hline 5 & 2,93 & 0,44 & 2,86 & 0,46 & $\underline{5,21}$ & $\underline{2,16}$ & 10,53 & 1,96 & 32,87 & 6,50 & 8,03 & 1,47 \\
\hline 6 & 2,48 & 0,43 & 2,91 & 0,46 & $\underline{2,70}$ & $\underline{0,40}$ & 10,36 & 1,90 & 51,80 & 11,19 & 26,52 & 13,03 \\
\hline 7 & 2,34 & 0,32 & 2,81 & 0,40 & 2,53 & $\underline{0,56}$ & 9,68 & 1,75 & 55,23 & 13,34 & 36,82 & 12,88 \\
\hline 8 & 2,92 & 0,44 & 2,88 & 0,51 & 2,67 & $\underline{0,36}$ & 12,92 & 6,49 & 41,50 & 12,12 & 20,30 & 8,63 \\
\hline 9 & 2,94 & 0,49 & 2,33 & 0,44 & 2,10 & $\underline{0,26}$ & 7,56 & 1,09 & 45,47 & 10,59 & 21,52 & 7,70 \\
\hline 10 & 2,37 & 0,36 & 1,94 & 0,24 & $\underline{2,30}$ & $\underline{0,38}$ & 5,48 & 0,89 & 24,66 & 5,84 & 8,03 & 1,70 \\
\hline 11 & 2,25 & 0,38 & 2,43 & 0,39 & $\underline{2,90}$ & $\underline{0,70}$ & 12,79 & 2,11 & 52,99 & 11,43 & 14,24 & 4,40 \\
\hline 12 & 2,84 & 0,46 & 2,21 & 0,34 & $\underline{2,04}$ & $\underline{0,33}$ & 10,36 & 2,18 & 44,08 & 9,70 & 13,79 & 4,73 \\
\hline \multicolumn{13}{|l|}{ Arrow } \\
\hline 1 & 2,37 & 0,26 & 2,60 & 0,44 & 2,56 & 0,37 & 12,17 & 3,41 & 39,23 & 7,55 & 19,17 & 4,54 \\
\hline 2 & 2,65 & 0,34 & 2,27 & 0,25 & 2,69 & 0,29 & 7,92 & 1,09 & 37,47 & 6,85 & 19,47 & 5,52 \\
\hline 3 & 2,80 & 0,32 & 3,19 & 0,48 & 2,88 & 0,41 & 11,21 & 1,43 & 45,73 & 7,26 & 12,80 & 2,98 \\
\hline 4 & 2,39 & 0,28 & 2,67 & 0,29 & 3,84 & 1,12 & 9,98 & 1,24 & 55,14 & 8,78 & 30,08 & 8,66 \\
\hline 5 & 2,91 & 0,35 & 2,99 & 0,36 & 2,57 & 0,30 & 10,83 & 1,57 & 40,01 & 6,71 & 12,50 & 2,96 \\
\hline 6 & 2,83 & 0,28 & 3,22 & 0,38 & 3,36 & 0,45 & 10,18 & 1,33 & 48,60 & 7,68 & 13,48 & 2,78 \\
\hline \multicolumn{13}{|l|}{ Score } \\
\hline 7 & 2,35 & 0,24 & $\underline{2,48}$ & $\underline{0,22}$ & $\underline{2,49}$ & $\underline{0,31}$ & $\underline{7,64}$ & $\underline{0,83}$ & 42,55 & 5,88 & 14,29 & 2,59 \\
\hline 8 & 2,92 & 0,28 & 2,67 & 0,23 & 2,27 & $\underline{0,17}$ & $\underline{8,33}$ & $\underline{2,14}$ & 32,34 & 5,21 & 19,76 & 5,08 \\
\hline 9 & 2,51 & 0,20 & 2,68 & 0,23 & $\underline{3,02}$ & $\underline{0,27}$ & 12,00 & 1,15 & 52,72 & 6,01 & 16,06 & 3,13 \\
\hline 10 & 2,93 & 0,29 & $\underline{3,71}$ & $\underline{0,56}$ & $\underline{4,50}$ & $\underline{1,05}$ & 13,61 & $\underline{1,47}$ & 48,36 & 7,20 & 22,99 & 5,58 \\
\hline
\end{tabular}

Fonte: Fernando Carvalheiro Reiser (2019)

Post hoc: $\mathrm{p}<0.05$ em itálico/sublinhado

No índice ED-TBL (tabela 27), não houve efeito no APA5 do bloco $\left(F_{11,520}=1.3\right.$; $\mathrm{p}=0.2)$, flecha $\left(F_{5,520}=0.6 ; \mathrm{p}=0.8\right)$ e escore $\left(F_{3,520}=1.0 ; \mathrm{p}=0.4\right)$. No APA4, não houve efeito do bloco $\left(F_{11,520}=1.4 ; \mathrm{p}=0.2\right)$, flecha $\left(F_{5,520}=0.3 ; \mathrm{p}=0.9\right)$ e escore $\left(F_{3,520}=1.4\right.$; $\mathrm{p}=0.3)$. No APA3, houve efeito no bloco $\left(F_{11,520}=2.2 ; \mathrm{p}=0.01\right)$; com maior modulação no 4 do que $1,2,8,10-12$, não houve efeito nas flechas $\left(F_{5,520}=1.1 ; \mathrm{p}=0.4\right)$, houve efeito no escore $\left(F_{3,520}=3.5 ; \mathrm{p}<0.01\right)$, maior no 10 em comparação ao 8 e 7 ( $\left.\mathrm{p}<0.01\right)$. No APA2, não houve efeito nos Blocos $\left(F_{11,520}=1.6 ; \mathrm{p}=0.1\right)$ e flechas $\left(F_{5,520}=0.6 ; \mathrm{p}=0.7\right)$, mas houve no escore $\left(F_{3,520}=5.4 ; \mathrm{p}<0.01\right)$ maior no escore 10 comparado ao 9,8 e 7 $(\mathrm{p}=0.03)$. No APA1, não houve efeito no bloco $\left(F_{11,520}=1.2 ; \mathrm{p}=0.32\right)$, e flecha $\left(F_{5,520}=1.5 ; \mathrm{p}=0.2\right)$, mas houve no escore $\left(F_{3,520}=3.9 ; \mathrm{p}=0.01\right)$, sem efeito no post hoc. No APC1, não houve efeito no bloco $\left(F_{11,520}=1.5 ; \mathrm{p}=0.2\right)$ e flecha $\left(F_{5,520}=0.4 ; \mathrm{p}=0.8\right)$, mas houve no escore $\left(F_{3,520}=4.4 ; \mathrm{p}<0.01\right)$, maior no 10 comparado ao 7 ( $\left.\mathrm{p}=0.03\right)$. 
Tabela 27. Inibição reciproca do par ED-TBL com Média e EP

\begin{tabular}{|c|c|c|c|c|c|c|c|c|c|c|c|c|}
\hline \multirow[t]{2}{*}{ Ends } & \multicolumn{2}{|c|}{$\begin{array}{l}\text { ED-TBL } \\
\text { (APA5) }\end{array}$} & \multicolumn{2}{|c|}{$\begin{array}{l}\text { ED-TBL } \\
\text { (APA4) }\end{array}$} & \multicolumn{2}{|c|}{$\begin{array}{l}\text { ED-TBL } \\
\text { (APA3) }\end{array}$} & \multicolumn{2}{|c|}{$\begin{array}{l}\text { ED-TBL } \\
\text { (APA2) }\end{array}$} & \multicolumn{2}{|c|}{$\begin{array}{c}\text { ED-TBL } \\
\text { (APA1) }\end{array}$} & \multicolumn{2}{|c|}{$\begin{array}{c}\text { ED-TBL } \\
\text { (APC1) }\end{array}$} \\
\hline & Mean & SE & Mean & SE & Mean & SE & Mean & SE & Mean & SE & Mean & SE \\
\hline 1 & 4,06 & 0,50 & 5,39 & 0,95 & $\underline{5,35}$ & $\underline{0,93}$ & 7,91 & 1,16 & 42,36 & 9,44 & 20,30 & 3,83 \\
\hline 2 & 4,83 & 0,79 & 5,48 & 0,86 & $\underline{6,08}$ & $\underline{0,99}$ & 16,47 & 4,85 & 78,07 & 14,68 & 39,70 & 8,48 \\
\hline 3 & 6,48 & 1,33 & 8,34 & 1,47 & 7,52 & 1,61 & 17,85 & 2,82 & 89,02 & 17,68 & 71,06 & 16,60 \\
\hline 4 & 15,18 & 5,13 & 19,67 & 7,49 & 23,87 & 11,48 & 20,03 & 8,19 & 105,72 & 24,61 & 79,39 & 19,72 \\
\hline 5 & 8,14 & 2,30 & 9,26 & 3,32 & 9,22 & 3,67 & 13,03 & 2,57 & 86,95 & 21,77 & 66,52 & 17,85 \\
\hline 6 & 6,37 & 1,44 & 5,75 & 0,99 & 5,70 & 0,98 & 12,86 & 2,25 & 110,31 & 23,24 & 76,67 & 18,74 \\
\hline 7 & 14,40 & 8,39 & 9,41 & 2,87 & 10,63 & 4,51 & 14,42 & 3,01 & 116,37 & 22,60 & 82,73 & 17,46 \\
\hline 8 & 5,48 & 1,04 & 4,88 & 0,88 & $\underline{4,55}$ & $\underline{0,79}$ & 18,65 & 6,55 & 132,12 & 24,04 & 91,97 & 19,61 \\
\hline 9 & 5,00 & 1,02 & 6,41 & 1,44 & 5,70 & 1,06 & 10,98 & 1,66 & 87,30 & 19,12 & 52,12 & 12,53 \\
\hline 10 & 5,55 & 1,18 & 4,90 & 0,80 & $\underline{5,26}$ & $\underline{1,10}$ & 8,50 & 1,30 & 88,11 & 17,76 & 68,48 & 14,97 \\
\hline 11 & 4,78 & 1,03 & 4,48 & 0,75 & $\underline{5,35}$ & $\underline{1,11}$ & 12,06 & 1,92 & 91,28 & 17,13 & 81,36 & 16,30 \\
\hline 12 & 4,90 & 0,95 & 5,40 & 1,10 & $\underline{5,55}$ & $\underline{1,21}$ & 9,47 & 1,55 & 71,75 & 18,38 & 72,88 & 15,51 \\
\hline \multicolumn{13}{|l|}{ Arrow } \\
\hline 1 & 10,05 & 4,33 & 7,60 & 1,94 & 7,73 & 1,92 & 15,86 & 3,71 & 96,08 & 14,89 & 67,35 & 11,66 \\
\hline 2 & 6,34 & 1,66 & 5,80 & 0,87 & 9,78 & 5,44 & 12,56 & 4,07 & 66,41 & 12,02 & 59,39 & 10,03 \\
\hline 3 & 6,00 & 0,89 & 8,59 & 3,00 & 6,10 & 0,94 & 12,83 & 1,51 & 90,06 & 13,36 & 59,32 & 10,64 \\
\hline 4 & 5,15 & 0,66 & 6,16 & 1,08 & 8,54 & 2,32 & 12,11 & 1,37 & 101,87 & 15,26 & 68,64 & 12,34 \\
\hline 5 & 7,91 & 1,93 & 8,82 & 2,02 & 7,35 & 1,31 & 13,48 & 2,51 & 82,95 & 12,43 & 69,77 & 11,78 \\
\hline 6 & 7,14 & 1,37 & 7,71 & 1,75 & 7,89 & 1,93 & 14,27 & 1,73 & 112,32 & 15,21 & 77,12 & 10,90 \\
\hline \multicolumn{13}{|l|}{ Score } \\
\hline 7 & 5,46 & 1,42 & 5,48 & 1,46 & $\underline{4,84}$ & $\underline{0,87}$ & $\underline{7,63}$ & $\underline{0,88}$ & 69,08 & 9,86 & 44,34 & $\underline{8,01}$ \\
\hline 8 & 5,93 & 0,62 & 5,56 & 0,52 & $\underline{6,00}$ & $\underline{0,57}$ & 11,74 & 2,24 & 83,59 & 11,94 & 66,80 & 9,68 \\
\hline 9 & 6,80 & 0,96 & 8,50 & 1,88 & 7,53 & 1,39 & 14,09 & $\underline{1,22}$ & 104,65 & 10,09 & 73,37 & 8,04 \\
\hline 10 & 11,21 & 4,02 & 10,53 & 1,57 & 14,87 & $\underline{5,28}$ & 22,01 & $\underline{4,32}$ & 106,27 & 13,75 & $\underline{82,45}$ & $\underline{11,04}$ \\
\hline
\end{tabular}

Fonte: Fernando Carvalheiro Reiser (2019)

Post hoc: $\mathrm{p}<0.05$ em itálico/sublinhado

\subsection{3 Índice Glenoumeral-Escápula}

O índice PMC-SAD (tabela 28), não apresentou efeito no APA5 do bloco $\left(F_{11,520}=0.7 ; \mathrm{p}=0.7\right)$, flecha $\left(F_{5,520}=0.7 ; \mathrm{p}=0.7\right)$ e escore $\left(F_{3,520}=0.6 ; \mathrm{p}=0.6\right)$. Neste índice não houve efeito no APA4 do bloco $\left(F_{11,520}=0.7 ; \mathrm{p}=0.7\right)$, flecha $\left(F_{5,520}=0.6 ; \mathrm{p}=0.7\right)$, e escore $\left(F_{3,520}=0.1 ; \mathrm{p}=0.9\right)$. Não houve efeito no APA3 do bloco $\left(F_{11,520}=0.5 ; \mathrm{p}=0.9\right)$, flecha $\left(F_{5,520}=0.2 ; \mathrm{p}=0.9\right)$ e escore $\left(F_{3,520}=0.4 ; \mathrm{p}=0.8\right)$. Não houve efeito no APA2 do bloco $\left(F_{11,520}=0.6 ; \mathrm{p}=0.8\right)$, flecha $\left(F_{5,520}=0.3 ; \mathrm{p}=0.9\right)$ e escore $\left(F_{3,520}=1.2 ; \mathrm{p}=0.3\right)$. Não houve efeito no APA1 do bloco $\left(F_{11,520}=0.4 ; \mathrm{p}=0.9\right)$, flecha $\left(F_{5,520}=0.4 ; \mathrm{p}=0.8\right)$ e escore $\left(F_{3,520}=1.4 ; \mathrm{p}=0.2\right)$. Não houve efeito no APC1 do bloco $\left(F_{11,520}=0.9 ; \mathrm{p}=0.5\right)$, flecha $\left(F_{5,520}=0.9 ; \mathrm{p}=0.5\right)$ e escore $\left(F_{3,520}=1.1 ; \mathrm{p}=0.4\right)$. 
Tabela 28. Inibição reciproca do par PMC-SAD com Média e EP

\begin{tabular}{|c|c|c|c|c|c|c|c|c|c|c|c|c|}
\hline \multirow[t]{2}{*}{ Ends } & \multicolumn{2}{|c|}{$\begin{array}{c}\text { PMC-SAD } \\
\text { (APA5) }\end{array}$} & \multicolumn{2}{|c|}{$\begin{array}{c}\text { PMC-SAD } \\
\text { (APA4) }\end{array}$} & \multicolumn{2}{|c|}{$\begin{array}{c}\text { PMC-SAD } \\
\text { (APA3) }\end{array}$} & \multicolumn{2}{|c|}{$\begin{array}{c}\text { PMC-SAD } \\
\text { (APA2) }\end{array}$} & \multicolumn{2}{|c|}{$\begin{array}{c}\text { PMC-SAD } \\
\text { (APA1) }\end{array}$} & \multicolumn{2}{|c|}{$\begin{array}{c}\text { PMC-SAD } \\
\text { (APC1) }\end{array}$} \\
\hline & Mean & SE & Mean & SE & Mean & SE & Mean & SE & Mean & SE & Mean & SE \\
\hline 1 & 1,36 & 0,20 & 1,65 & 0,28 & 1,81 & 0,25 & 7,67 & 1,88 & 41,43 & 9,73 & 27,12 & 7,99 \\
\hline 2 & 12,59 & 6,14 & 19,96 & 8,00 & 17,64 & 5,99 & 32,18 & 9,76 & 48,93 & 12,25 & 36,67 & 10,08 \\
\hline 3 & 14,54 & 5,89 & 5,98 & 2,16 & 12,28 & 5,29 & 22,42 & 4,91 & 58,89 & 12,11 & 30,91 & 8,54 \\
\hline 4 & 5,45 & 3,12 & 2,47 & 1,28 & 5,15 & 3,78 & 13,09 & 3,77 & 52,29 & 10,74 & 52,88 & 12,31 \\
\hline 5 & 8,57 & 5,52 & 2,33 & 0,52 & 2,79 & 0,73 & 15,11 & 3,01 & 53,50 & 10,16 & 49,09 & 12,26 \\
\hline 6 & 17,47 & 8,06 & 12,71 & 7,57 & 8,56 & 4,37 & 14,70 & 4,23 & 47,69 & 11,66 & 39,55 & 11,09 \\
\hline 7 & 3,56 & 1,66 & 5,62 & 1,98 & 9,18 & 4,24 & 15,23 & 4,75 & 24,30 & 6,63 & 23,48 & 9,08 \\
\hline 8 & 3,45 & 1,79 & 5,16 & 2,14 & 4,12 & 1,44 & 14,30 & 3,41 & 46,56 & 8,67 & 39,39 & 9,23 \\
\hline 9 & 10,56 & 5,64 & 7,10 & 2,80 & 13,27 & 6,23 & 19,59 & 4,91 & 53,09 & 11,40 & 60,61 & 13,92 \\
\hline 10 & 4,63 & 2,88 & 1,86 & 0,30 & 1,85 & 0,27 & 12,94 & 2,86 & 56,54 & 10,47 & 36,36 & 9,06 \\
\hline 11 & 3,29 & 1,06 & 5,56 & 1,98 & 10,16 & 6,07 & 14,98 & 4,05 & 33,69 & 7,69 & 31,82 & 7,41 \\
\hline 12 & 14,30 & 6,04 & 9,16 & 3,83 & 9,93 & 4,90 & 24,12 & 6,08 & 60,31 & 12,71 & 52,27 & 9,98 \\
\hline \multicolumn{13}{|l|}{ Arrow } \\
\hline 1 & 6,72 & 2,61 & 4,38 & 1,51 & 8,91 & 3,67 & 18,06 & 3,62 & 51,29 & 7,49 & 38,86 & 7,09 \\
\hline 2 & 12,30 & 4,68 & 9,06 & 3,89 & 6,52 & 1,99 & 12,92 & 2,65 & 36,69 & 6,03 & 32,12 & 6,64 \\
\hline 3 & 5,66 & 2,49 & 4,75 & 1,67 & 5,42 & 2,19 & 16,14 & 3,02 & 55,09 & 8,26 & 51,36 & 8,59 \\
\hline 4 & 13,25 & 4,24 & 11,35 & 4,05 & 8,67 & 2,93 & 15,98 & 3,74 & 45,40 & 6,76 & 41,06 & 7,27 \\
\hline 5 & 6,73 & 2,96 & 5,51 & 1,66 & 10,08 & 3,77 & 20,55 & 4,06 & 52,23 & 8,30 & 38,41 & 7,16 \\
\hline 6 & 5,24 & 1,99 & 4,72 & 1,38 & 8,78 & 3,15 & 19,52 & 3,48 & 47,91 & 7,52 & 38,26 & 6,69 \\
\hline \multicolumn{13}{|l|}{ Score } \\
\hline 7 & 12,94 & 4,10 & 6,64 & 1,75 & 5,38 & 1,67 & 14,78 & 3,04 & 36,90 & 6,59 & 28,51 & 5,35 \\
\hline 8 & 7,85 & 2,74 & 7,66 & 3,21 & 5,96 & 1,89 & 16,91 & 2,86 & 51,67 & 6,41 & 41,75 & 6,37 \\
\hline 9 & 7,62 & 1,99 & 5,11 & 1,14 & 10,16 & 2,82 & 15,07 & 2,07 & 53,54 & 5,23 & 47,73 & 5,46 \\
\hline 10 & 4,69 & 1,21 & 7,92 & 2,16 & 10,45 & 2,74 & 24,29 & 3,83 & 46,66 & 6,01 & 37,41 & 6,15 \\
\hline
\end{tabular}

Fonte: Fernando Carvalheiro Reiser (2019)

No índice GD-TS (tabela 29), não houve efeito APA5 do bloco $\left(F_{11,520}=1.4\right.$; $\mathrm{p}=0.2)$, flecha $\left(F_{5,520}=1.7 ; \mathrm{p}=0.1\right)$ e escore $\left(F_{3,520}=0.5 ; \mathrm{p}=0.6\right)$. Não houve efeito no APA4 do bloco $\left(F_{11,520}=1.4 ; \mathrm{p}=0.2\right)$, flecha $\left(F_{5,520}=1.1 ; \mathrm{p}=0.4\right)$ e escore $\left(F_{3,520}=0.6\right.$; $\mathrm{p}=0.6)$. Não houve efeito no APA3 para bloco $\left(F_{11,520}=0.5 ; \mathrm{p}=0.9\right)$, flecha $\left(F_{5,520}=0.5\right.$; $\mathrm{p}=0.7)$ e escore $\left(F_{3,520}=0.7 ; \mathrm{p}=0.5\right)$. Não houve efeito no APA2 do bloco $\left(F_{11,520}=1.5\right.$; $\mathrm{p}=0.2)$, flecha $\left(F_{5,520}=0.9 ; \mathrm{p}=0.5\right)$ e escore $\left(F_{3,520}=0.2 ; \mathrm{p}=0.9\right)$. Não houve efeito no APA1 do bloco $\left(F_{11,520}=1.8 ; \mathrm{p}=0.1\right)$, flecha $\left(F_{5,520}<0.01 ; \mathrm{p}=0.9\right)$ e escore $\left(F_{3,520}=2.2\right.$; $\mathrm{p}=0.1)$. Não houve efeito no APC1 para bloco $\left(F_{11,520}=1.2 ; \mathrm{p}=0.3\right)$, flecha $\left(F_{5,520}=0.2\right.$; $\mathrm{p}=0.9)$ e escore $\left(F_{3,520}=2.1 ; \mathrm{p}=0.1\right)$. 
Tabela 29. Inibição reciproca do par GD-TS com Média e EP

\begin{tabular}{|c|c|c|c|c|c|c|c|c|c|c|c|c|}
\hline \multirow[t]{2}{*}{ Ends } & \multicolumn{2}{|c|}{ GD-TS (APA5) } & \multicolumn{2}{|c|}{ GD-TS (APA4) } & \multicolumn{2}{|c|}{ GD-TS (APA3) } & \multicolumn{2}{|c|}{ GD-TS (APA2) } & \multicolumn{2}{|c|}{ GD-TS (APA1) } & \multicolumn{2}{|c|}{ GD-TS (APC1) } \\
\hline & Mean & SE & Mean & SE & Mean & SE & Mean & SE & Mean & SE & Mean & SE \\
\hline 1 & 4,01 & 0,55 & 3,80 & 0,52 & 4,61 & 0,95 & 8,82 & 3,42 & 48,49 & 15,32 & 49,24 & 16,10 \\
\hline 2 & 3,68 & 0,54 & 3,07 & 0,49 & 4,31 & 0,71 & 11,12 & 3,86 & 26,33 & 8,58 & 55,76 & 16,85 \\
\hline 3 & 6,55 & 1,56 & 6,59 & 1,53 & 6,74 & 1,42 & 8,85 & 2,85 & 33,46 & 7,58 & 57,27 & 12,13 \\
\hline 4 & 7,51 & 1,93 & 6,37 & 1,47 & 4,90 & 1,19 & 13,80 & 4,32 & 58,56 & 16,01 & 65,00 & 20,85 \\
\hline 5 & 7,01 & 1,21 & 8,08 & 1,71 & 6,42 & 1,15 & 16,89 & 4,85 & 56,97 & 16,14 & 61,06 & 17,81 \\
\hline 6 & 8,31 & 1,64 & 8,01 & 1,69 & 8,39 & 1,94 & 8,20 & 1,61 & 59,03 & 14,86 & 58,03 & 14,54 \\
\hline 7 & 10,80 & 3,92 & 14,46 & 7,30 & 8,32 & 2,95 & 8,03 & 1,90 & 31,71 & 9,47 & 36,82 & 13,04 \\
\hline 8 & 6,50 & 1,37 & 4,22 & 0,77 & 5,22 & 1,19 & 11,47 & 4,24 & 10,75 & 3,76 & 10,76 & 4,62 \\
\hline 9 & 5,18 & 1,05 & 7,07 & 1,39 & 7,94 & 1,36 & 11,38 & 4,49 & 26,96 & 9,35 & 36,67 & 12,55 \\
\hline 10 & 6,20 & 1,14 & 5,93 & 1,12 & 6,79 & 1,12 & 6,09 & 2,14 & 18,20 & 5,31 & 21,06 & 7,69 \\
\hline 11 & 5,20 & 1,27 & 5,75 & 1,08 & 5,19 & 0,95 & 5,59 & 0,90 & 29,22 & 10,01 & 33,03 & 10,70 \\
\hline 12 & 5,85 & 1,02 & 8,49 & 2,87 & 11,90 & 6,74 & 6,33 & 1,11 & 24,09 & 6,68 & 23,64 & 8,87 \\
\hline \multicolumn{13}{|l|}{ Arrow } \\
\hline 1 & 4,66 & 0,57 & 5,07 & 0,61 & 5,48 & 0,68 & 12,48 & 3,12 & 40,16 & 8,44 & 56,97 & 12,72 \\
\hline 2 & 4,66 & 0,60 & 5,03 & 0,67 & 4,37 & 0,49 & 8,88 & 2,24 & 34,16 & 8,00 & 38,03 & 9,17 \\
\hline 3 & 7,03 & 1,18 & 6,13 & 1,35 & 6,04 & 0,78 & 7,98 & 1,36 & 35,47 & 7,78 & 38,94 & 9,07 \\
\hline 4 & 5,67 & 0,79 & 7,02 & 1,60 & 9,04 & 3,44 & 10,88 & 2,29 & 35,07 & 6,89 & 38,03 & 8,30 \\
\hline 5 & 9,44 & 2,11 & 10,46 & 3,59 & 7,75 & 1,62 & 9,22 & 2,41 & 40,44 & 9,51 & 43,56 & 9,67 \\
\hline 6 & 6,93 & 1,06 & 7,21 & 1,13 & 7,67 & 1,24 & 8,85 & 2,09 & 26,60 & 6,40 & 38,64 & 8,95 \\
\hline \multicolumn{13}{|l|}{ Score } \\
\hline 7 & 7,77 & 1,53 & 8,25 & 2,68 & 6,99 & 1,20 & 9,90 & 2,33 & 48,36 & 8,96 & 62,63 & 11,10 \\
\hline 8 & 6,76 & 0,84 & 7,79 & 1,34 & 8,81 & 2,27 & 10,96 & 1,99 & 33,99 & 5,32 & 41,60 & 7,34 \\
\hline 9 & 5,70 & 0,67 & 5,37 & 0,63 & 5,06 & 0,56 & 7,39 & 1,23 & 37,66 & 5,94 & 41,97 & 6,66 \\
\hline 10 & 5,53 & 0,75 & 6,35 & 0,84 & 6,48 & 0,90 & 11,94 & 2,34 & 17,44 & 3,91 & 20,00 & 5,55 \\
\hline
\end{tabular}

Fonte: Fernando Carvalheiro Reiser (2019)

4.3.4 Índices mistos

Na tabela 30 está o índice Serrátil Anterior bilateral (SAD-SAnD). Neste índice, teve efeito no APA5 do bloco, $\left(F_{11,520}=8.3 ;\right.$ p $\left.<0.01\right)$, maior no 5 comparado ao $1-4,6-11$ $(\mathrm{p}<0.01)$, não teve efeito na flecha $\left(F_{5,520}=1.6 ; \mathrm{p}=0.2\right)$, no escore teve efeito $\left(F_{3,520}=3.0\right.$; $\mathrm{p}=0.03)$ maior no 10 comparado a $9(\mathrm{p}=0.05)$. No APA4 teve efeito do bloco $\left(F_{11,520}=6.7 ; \mathrm{p}<0.01\right)$; maior no 5 comparado a 1-4,6-11 ( $\left.<<0.01\right)$, não teve efeito da flecha $\left(F_{5,520}=2.0 ; \mathrm{p}=0.1\right)$, e escore $\left(F_{3,520}=1.5 ; \mathrm{p}=0.2\right)$. Houve efeito no APA do bloco $\left(F_{11,520}=2.6 ;\right.$ p $\left.<0.01\right)$ sem detecção no post hoc, não houve efeito na flecha $\left(F_{5,520}=0.8\right.$; $\mathrm{p}=0.5)$, e escore $\left(F_{3,520}=0.4 ; \mathrm{p}=0.7\right)$. Houve efeito no APA2 do bloco $\left(F_{11,520}=3.7\right.$; $\mathrm{p}<0.01)$ maior no 5 do que 1,4 e 7 ( $<<0.03)$. Não houve efeito na flecha $\left(F_{5,520}=0.7\right.$; $\mathrm{p}=0.6)$. Houve efeito no escore $\left(F_{3,520}=2.8 ; \mathrm{p}=0.04\right)$, maior no 10 do que 7 ( $\left.\mathrm{p}<0.01\right)$. No APA1 não houve efeito no bloco $\left(F_{11,520}=1.5 ; \mathrm{p}=0.1\right)$ e flecha $\left(F_{5,520}=0.7 ; \mathrm{p}=0.6\right)$, mas houve no escore $\left(F_{3,520}=4.9 ; \mathrm{p}<0.01\right)$, maior no 9 do que 8 e 7 ( $\left.<<0.01\right)$. Não houve 
efeito no APC1 do bloco $\left(F_{11,520}=1.7 ; \mathrm{p}=0.1\right)$ e flecha $\left(F_{5,520}=1.6 ; \mathrm{p}=0.2\right)$. Houve efeito no escore $\left(F_{3,520}=6.0 ; \mathrm{p}<0.01\right)$; maior no 10 e 9 comparado ao 7 e $8(\mathrm{p}<0.01)$.

Tabela 30. Inibição reciproca do par SAD-SAnD com Média e EP

\begin{tabular}{|c|c|c|c|c|c|c|c|c|c|c|c|c|}
\hline \multirow[t]{2}{*}{ Ends } & \multicolumn{2}{|c|}{$\begin{array}{l}\text { SAD-SAnD } \\
\text { (APA5) }\end{array}$} & \multicolumn{2}{|c|}{$\begin{array}{c}\text { SAD-SAnD } \\
\text { (APA4) }\end{array}$} & \multicolumn{2}{|c|}{$\begin{array}{c}\text { SAD-SAnD } \\
\text { (APA3) }\end{array}$} & \multicolumn{2}{|c|}{$\begin{array}{c}\text { SAD-SAnD } \\
\text { (APA2) }\end{array}$} & \multicolumn{2}{|c|}{$\begin{array}{c}\text { SAD-SAnD } \\
\text { (APA1) }\end{array}$} & \multicolumn{2}{|c|}{$\begin{array}{c}\text { SAD-SAnD } \\
\text { (APC1) }\end{array}$} \\
\hline & Mean & SE & Mean & SE & Mean & SE & Mean & SE & Mean & SE & Mean & SE \\
\hline 1 & 1,25 & $\underline{0,16}$ & $\underline{1,70}$ & $\underline{0,30}$ & 1,79 & 0,29 & 2,48 & 0,28 & 16,21 & 5,20 & 26,97 & 8,90 \\
\hline 2 & $\underline{1,72}$ & $\underline{0,29}$ & $\underline{1,49}$ & $\underline{0,20}$ & 2,51 & 0,85 & 4,85 & 2,29 & 13,56 & 3,99 & 16,21 & 7,00 \\
\hline 3 & $\underline{2,18}$ & $\underline{0,45}$ & $\underline{2,36}$ & $\underline{0,37}$ & 3,27 & 0,93 & 7,55 & 2,52 & 24,97 & 8,07 & 24,70 & 6,58 \\
\hline 4 & $\underline{2,23}$ & $\underline{0,45}$ & $\underline{1,58}$ & $\underline{0,28}$ & 2,03 & 0,28 & $\underline{3,20}$ & $\underline{0,51}$ & 25,29 & 7,91 & 23,48 & 7,90 \\
\hline 5 & 23,36 & $\underline{8,66}$ & $\underline{15,75}$ & $\underline{7,59}$ & 16,20 & 7,01 & $\underline{14,56}$ & $\underline{4,84}$ & 35,09 & 8,32 & 41,06 & 9,90 \\
\hline 6 & $\underline{5,87}$ & $\underline{3,88}$ & $\underline{2,12}$ & $\underline{0,30}$ & 1,87 & 0,28 & 5,83 & 1,23 & 30,16 & 8,65 & 21,06 & 6,69 \\
\hline 7 & $\underline{1,46}$ & $\underline{0,26}$ & $\underline{2,55}$ & $\underline{0,44}$ & 2,11 & 0,32 & $\underline{3,67}$ & $\underline{0,74}$ & 16,92 & 6,09 & 13,64 & 5,27 \\
\hline 8 & $\underline{1,85}$ & $\underline{0,30}$ & $\underline{2,65}$ & $\underline{0,52}$ & 2,25 & 0,44 & 5,36 & 1,87 & 21,69 & 5,70 & 29,09 & 8,76 \\
\hline 9 & $\underline{2,03}$ & $\underline{0,33}$ & $\underline{2,87}$ & $\underline{0,57}$ & 9,34 & 5,92 & 7,18 & 3,24 & 9,32 & 3,00 & 22,42 & 9,21 \\
\hline 10 & $\underline{1,79}$ & $\underline{0,29}$ & $\underline{1,89}$ & $\underline{0,30}$ & 2,14 & 0,30 & 5,59 & 1,66 & 29,97 & 8,28 & 22,58 & 7,78 \\
\hline 11 & $\underline{1,63}$ & $\underline{0,21}$ & $\underline{2,36}$ & $\underline{0,54}$ & 7,74 & 5,88 & 5,98 & 2,36 & 22,01 & 6,10 & 24,85 & 9,42 \\
\hline 12 & 10,87 & 5,42 & $\underline{3,48}$ & $\underline{1,23}$ & 1,91 & 0,30 & 5,03 & 1,28 & 24,29 & 7,51 & 23,48 & 8,48 \\
\hline \multicolumn{13}{|l|}{ Arrow } \\
\hline 1 & 4,82 & 2,46 & 5,36 & 3,24 & 7,70 & 3,75 & 7,64 & 2,30 & 18,96 & 4,44 & 14,55 & 3,72 \\
\hline 2 & 6,51 & 3,41 & 4,20 & 1,84 & 4,08 & 2,35 & 3,90 & 1,15 & 19,25 & 4,20 & 24,24 & 5,87 \\
\hline 3 & 2,95 & 1,19 & 2,46 & 0,33 & 3,01 & 1,08 & 5,53 & 1,26 & 26,23 & 4,97 & 35,76 & 7,56 \\
\hline 4 & 6,67 & 2,79 & 2,04 & 0,25 & 2,00 & 0,22 & 4,15 & 0,64 & 22,57 & 5,09 & 22,12 & 5,61 \\
\hline 5 & 3,81 & 1,60 & 2,86 & 0,64 & 6,75 & 3,07 & 7,65 & 1,91 & 25,67 & 5,63 & 15,83 & 4,12 \\
\hline 6 & 3,37 & 1,45 & 3,49 & 1,09 & 3,05 & 0,71 & 6,77 & 1,80 & 22,07 & 4,48 & 32,27 & 6,36 \\
\hline \multicolumn{13}{|l|}{ Score } \\
\hline 7 & 7,29 & 2,61 & 3,88 & 1,01 & 3,42 & 0,74 & $\underline{4,07}$ & $\underline{1,00}$ & $\underline{14,89}$ & $\underline{3,69}$ & $\underline{10,69}$ & $\underline{3,04}$ \\
\hline 8 & 4,60 & 1,75 & 4,32 & 2,08 & 4,32 & 1,64 & 5,81 & 1,36 & $\underline{14,71}$ & $\underline{3,19}$ & $\underline{16,46}$ & $\underline{4,02}$ \\
\hline 9 & $\underline{1,65}$ & $\underline{0,16}$ & 2,08 & 0,22 & 4,64 & 2,08 & 5,13 & 0,92 & $\underline{32,23}$ & $\underline{4,07}$ & $\underline{33,67}$ & $\underline{4,86}$ \\
\hline 10 & $\underline{7,16}$ & $\underline{3,08}$ & 3,91 & 1,58 & 5,41 & 2,20 & $\underline{9,80}$ & $\underline{2,16}$ & 24,77 & 4,20 & $\underline{33,74}$ & $\underline{5,83}$ \\
\hline
\end{tabular}

Fonte: Fernando Carvalheiro Reiser (2019)

Post hoc: $\mathrm{p}<0.05$ em itálico/sublinhado

No índice que segura o arco SAnD-DPnD (tabela 31), houve efeito no APA5 do bloco $\left(F_{11,520}=2.8 ; \mathrm{p}<0.01\right)$; maior no 5 em comparação aos blocos 1,2,7,11 (p<0.05), não houve efeito na flecha $\left(F_{5,520}=0.6 ; \mathrm{p}=0.7\right)$, mas houve no escore $(F 3,520=4.3$; $\mathrm{p}<0.01)$ maior no 10 em comparação ao $9(\mathrm{p}<0.01)$. Neste índice, não houve efeito no APA4 do bloco $\left(F_{11,520}=1.6 ; \mathrm{p}=0.1\right)$ e flecha $\left(F_{5,520}=1.0 ; \mathrm{p}=0.4\right)$, houve efeito no escore $\left(F_{3,520}=3.3 ;\right.$ p=0.02) sem detecção no post hoc. No APA3, houve efeito no bloco $\left(F_{11,520}=2.0 ; \mathrm{p}=0.03\right)$, sem detecção no post hoc. Não houve efeito na flecha $\left(F_{5,520}=1.2\right.$; $\mathrm{p}=0.3)$, mas houve no escore $\left(F_{3,520}=4.2 ; \mathrm{p}<0.01\right)$ maior no escore 10 do que $9(\mathrm{p}<0.01)$. Não houve efeito no APA2 do bloco $\left(F_{11,520}=1.6 ; \mathrm{p}=0.1\right)$, flecha $\left(F_{5,520}=0.7 ; \mathrm{p}=0.6\right)$ e escore $\left(F_{3,520}=1.4 ; \mathrm{p}=0.2\right)$. No APA1 não houve efeito para bloco $\left(F_{11,520}=0.7 ; \mathrm{p}=0.7\right)$, 
flecha $\left(F_{5,520}=0.4 ; \mathrm{p}=0.9\right)$ e escore $\left(F_{3,520}=0.8 ; \mathrm{p}=0.5\right)$. No APC1 não houve efeito do bloco $\left(F_{11,520}=0.3 ; \mathrm{p}=0.9\right)$, flecha $\left(F_{5,520}=1.1 ; \mathrm{p}=0.3\right)$ e escore $\left(F_{3,520}=2.6 ; \mathrm{p}=0.1\right)$.

Tabela 31. Inibição reciproca do par SAnD-DPnD com Média e EP

\begin{tabular}{|c|c|c|c|c|c|c|c|c|c|c|c|c|}
\hline \multirow[t]{2}{*}{ Ends } & \multicolumn{2}{|c|}{$\begin{array}{c}\text { SAnD-DPnD } \\
\text { (APA5) }\end{array}$} & \multicolumn{2}{|c|}{$\begin{array}{c}\text { SAnD-DPnD } \\
\text { (APA4) }\end{array}$} & \multicolumn{2}{|c|}{$\begin{array}{c}\text { SAnD-DPnD } \\
\text { (APA3) }\end{array}$} & \multicolumn{2}{|c|}{$\begin{array}{c}\text { SAnD-DPnD } \\
\text { (APA2) }\end{array}$} & \multicolumn{2}{|c|}{$\begin{array}{c}\text { SAnD-DPnD } \\
\text { (APA1) }\end{array}$} & \multicolumn{2}{|c|}{$\begin{array}{c}\text { SAnD-DPnD } \\
\text { (APC1) }\end{array}$} \\
\hline & Mean & SE & Mean & $\mathrm{SE}$ & Mean & SE & Mean & SE & Mean & SE & Mean & SE \\
\hline 1 & 6,08 & 1,52 & 8,61 & 4,60 & 7,72 & 3,07 & 8,82 & 3,42 & 22,23 & 6,52 & 18,33 & 6,25 \\
\hline 2 & 9,05 & 2,62 & 10,73 & 2,78 & 17,91 & 5,98 & 11,12 & 3,86 & 24,83 & 7,19 & 28,48 & 7,09 \\
\hline 3 & 11,24 & 3,97 & 8,54 & 2,41 & 9,51 & 2,55 & 8,85 & 2,85 & 26,51 & 9,98 & 29,85 & 8,33 \\
\hline 4 & 13,33 & 5,15 & 13,20 & 4,49 & 17,08 & 6,57 & 13,80 & 4,32 & 20,59 & 6,70 & 32,12 & 8,39 \\
\hline 5 & $\underline{27,54}$ & $\underline{8,92}$ & 21,23 & 7,63 & 21,43 & 7,01 & 16,89 & 4,85 & 33,29 & 7,96 & 22,58 & 6,27 \\
\hline 6 & $\overline{10,26}$ & $\overline{3,62}$ & 7,30 & 1,38 & 9,13 & 2,64 & 8,20 & 1,61 & 29,24 & 8,24 & 27,42 & 6,68 \\
\hline 7 & $\underline{5,97}$ & $\underline{1,13}$ & 8,08 & 1,52 & 8,36 & 1,95 & 8,03 & 1,90 & 26,37 & 7,94 & 19,24 & 6,34 \\
\hline 8 & 10,22 & 3,70 & 13,75 & 6,05 & 16,94 & 6,83 & 11,47 & 4,24 & 17,11 & 5,86 & 33,79 & 8,77 \\
\hline 9 & 10,33 & 2,98 & 13,44 & 5,11 & 11,23 & 3,61 & 11,38 & 4,49 & 14,73 & 4,80 & 31,06 & 9,98 \\
\hline 10 & 9,80 & 3,90 & 8,24 & 3,20 & 7,86 & 2,76 & 6,09 & 2,14 & 19,85 & 6,46 & 26,67 & 6,66 \\
\hline 11 & $\underline{5,25}$ & $\underline{0,97}$ & 5,49 & 0,95 & 5,02 & 0,86 & 5,59 & 0,90 & 24,87 & 7,26 & 25,61 & 7,58 \\
\hline 12 & 9,41 & 4,23 & 11,61 & 4,44 & 10,81 & 4,28 & 6,33 & 1,11 & 23,52 & 7,29 & 25,76 & 7,79 \\
\hline \multicolumn{13}{|l|}{ Arrow } \\
\hline 1 & 12,84 & 3,55 & 12,16 & 3,95 & 13,31 & 3,97 & 12,48 & 3,12 & 21,19 & 5,02 & 24,02 & 5,94 \\
\hline 2 & 9,76 & 3,03 & 14,00 & 3,51 & 14,67 & 3,91 & 8,88 & 2,24 & 20,14 & 4,73 & 22,35 & 4,48 \\
\hline 3 & 11,83 & 3,28 & 8,82 & 2,34 & 10,18 & 2,55 & 7,98 & 1,36 & 23,15 & 4,95 & 19,24 & 4,16 \\
\hline 4 & 10,37 & 2,80 & 12,08 & 2,77 & 12,72 & 2,79 & 10,88 & 2,29 & 29,22 & 6,10 & 29,02 & 5,32 \\
\hline 5 & 9,02 & 2,45 & 7,75 & 1,53 & 10,91 & 2,97 & 9,22 & 2,41 & 24,26 & 5,28 & 28,56 & 5,45 \\
\hline 6 & 10,42 & 2,25 & 10,29 & 3,07 & 9,71 & 2,57 & 8,85 & 2,09 & 23,62 & 4,67 & 37,27 & 6,37 \\
\hline \multicolumn{13}{|l|}{ Score } \\
\hline 7 & 8,62 & 1,98 & 8,59 & 1,91 & 10,18 & 2,48 & 9,90 & 2,33 & 22,79 & 4,30 & 22,63 & 4,14 \\
\hline 8 & 12,46 & 2,87 & 13,33 & 3,25 & 14,09 & 2,88 & 10,96 & 1,99 & 30,23 & 4,64 & 35,24 & 5,13 \\
\hline 9 & $\underline{7,01}$ & $\underline{1,11}$ & 7,53 & 1,45 & $\underline{7,29}$ & $\underline{1,13}$ & 7,39 & 1,23 & 21,34 & 3,71 & 21,02 & 3,24 \\
\hline 10 & $\underline{17,36}$ & $\underline{3,90}$ & 16,04 & 3,08 & $\underline{19,24}$ & $\underline{4,38}$ & 11,94 & 2,34 & 19,31 & 3,83 & 30,00 & 5,28 \\
\hline
\end{tabular}

No índice os extensores do ombro GD-DPnD (tabela 32). Não houve efeito neste índice, no APA5, do bloco $\left(F_{11,520}=0.9 ; \mathrm{p}=0.6\right)$ e flecha $\left(F_{5,520}=1.0 ; \mathrm{p}=0.4\right)$ e houve efeito no escore $\left(F_{3,520}=3.5 ; \mathrm{p}=0.01\right)$ maior no 10 em comparação ao 9 e 7 ( $\left.\mathrm{p}<0.05\right)$. Não houve efeito no APA4 do bloco $\left(F_{11,520}=0.7 ; \mathrm{p}=0.8\right)$, flecha $\left(F_{5,520}=0.9 ; \mathrm{p}=0.5\right)$ e escore $\left(F_{3,520}=2.1 ; \mathrm{p}=0.1\right)$. Não houve efeito no APA3 do bloco $\left(F_{11,520}=1.5 ; \mathrm{p}=0.1\right)$, flecha $\left(F_{5,520}=1.1 ; \mathrm{p}=0.4\right)$, mas houve no escore $\left(F_{3,520}=2.6 ; \mathrm{p}=0.05\right)$; maior no escore $10 \mathrm{em}$ comparação ao 9 ( $<<0.02)$. Não houve efeito no APA2 do bloco $\left(F_{11,520}=1.1 ; \mathrm{p}=0.3\right)$, flecha $\left(F_{5,520}=0.8 ; \mathrm{p}=0.6\right)$, e escore $\left(F_{3,520}=1.3 ; \mathrm{p}=0.3\right)$. Não houve efeito no APA1 do bloco $\left(F_{11,520}=0.5 ; \mathrm{p}=0.9\right)$, flecha $\left(F_{5,520}=0.3 ; \mathrm{p}=0.9\right)$, e escore $\left(F_{3,520}=1.4 ; \mathrm{p}=0.3\right)$. Não 
houve efeito no APC1 do bloco $\left(F_{11,520}=0.6 ; \mathrm{p}=0.8\right)$, flecha $\left(F_{5,520}=0.7 ; \mathrm{p}=0.6\right)$, e escore $\left(F_{3,520}=2.0 ; \mathrm{p}=0.1\right)$.

Tabela 32. Inibição reciproca do par GD-DPnD com Média e EP

\begin{tabular}{|c|c|c|c|c|c|c|c|c|c|c|c|c|}
\hline \multirow[t]{2}{*}{ Ends } & \multicolumn{2}{|c|}{$\begin{array}{l}\text { GD-DPnD } \\
\text { (APA5) }\end{array}$} & \multicolumn{2}{|c|}{$\begin{array}{l}\text { GD-DPnD } \\
\text { (APA4) }\end{array}$} & \multicolumn{2}{|c|}{$\begin{array}{c}\text { GD-DPnD } \\
\text { (APA3) }\end{array}$} & \multicolumn{2}{|c|}{$\begin{array}{l}\text { GD-DPnD } \\
\text { (APA2) }\end{array}$} & \multicolumn{2}{|c|}{$\begin{array}{l}\text { GD-DPnD } \\
\text { (APA1) }\end{array}$} & \multicolumn{2}{|c|}{$\begin{array}{c}\text { GD-DPnD } \\
\text { (APC1) }\end{array}$} \\
\hline & Mean & SE & Mean & SE & Mean & SE & Mean & SE & Mean & SE & Mean & SE \\
\hline 1 & 6,40 & 1,55 & 9,21 & 4,65 & 7,71 & 3,10 & 8,53 & 3,49 & 24,24 & 6,38 & 21,21 & 4,96 \\
\hline 2 & 9,31 & 2,65 & 10,60 & 2,83 & 18,39 & 6,01 & 11,61 & 3,93 & 28,96 & 7,63 & 38,48 & 9,17 \\
\hline 3 & 11,54 & 3,98 & 8,41 & 2,42 & 9,57 & 2,54 & 10,50 & 2,90 & 37,66 & 9,82 & 45,61 & 10,63 \\
\hline 4 & 13,52 & 5,11 & 13,15 & 4,54 & 17,07 & 6,68 & 14,70 & 4,45 & 26,48 & 7,06 & 40,00 & 8,83 \\
\hline 5 & 9,80 & 3,60 & 7,90 & 1,61 & 7,77 & 1,35 & 8,65 & 1,48 & 28,59 & 7,23 & 37,58 & 8,30 \\
\hline 6 & 10,78 & 3,63 & 7,63 & 1,45 & 9,76 & 2,69 & 9,89 & 1,75 & 33,24 & 8,22 & 34,55 & 7,82 \\
\hline 7 & 7,77 & 1,95 & 10,24 & 2,68 & 9,08 & 2,01 & 13,00 & 3,17 & 35,89 & 9,36 & 28,94 & 8,09 \\
\hline 8 & 10,39 & 3,71 & 13,75 & 6,08 & 16,69 & 6,83 & 12,53 & 4,26 & 22,48 & 6,18 & 36,21 & 8,94 \\
\hline 9 & 10,57 & 2,99 & 13,45 & 5,13 & 11,66 & 3,63 & 13,98 & 4,47 & 26,70 & 7,88 & 46,97 & 11,74 \\
\hline 10 & 10,05 & 3,94 & 8,85 & 3,18 & 8,71 & 2,74 & 8,35 & 2,25 & 27,04 & 6,98 & 31,67 & 6,25 \\
\hline 11 & 5,65 & 0,94 & 5,64 & 0,92 & 5,30 & 0,84 & 6,92 & 1,23 & 28,00 & 7,11 & 31,06 & 7,42 \\
\hline 12 & 10,77 & 4,27 & 12,00 & 4,48 & 11,03 & 4,28 & 6,74 & 1,19 & 26,32 & 7,31 & 24,85 & 6,52 \\
\hline \multicolumn{13}{|l|}{ Arrow } \\
\hline 1 & 10,80 & 2,84 & 8,83 & 2,37 & 11,08 & 3,31 & 12,10 & 2,80 & 27,72 & 5,58 & 30,76 & 6,62 \\
\hline 2 & 7,08 & 1,25 & 12,48 & 3,12 & 12,56 & 3,19 & 8,70 & 2,04 & 22,50 & 4,88 & 32,12 & 5,67 \\
\hline 3 & 11,74 & 3,18 & 9,78 & 2,62 & 9,61 & 2,38 & 9,80 & 1,53 & 28,42 & 5,19 & 30,38 & 5,31 \\
\hline 4 & 10,56 & 2,80 & 12,12 & 2,78 & 12,80 & 2,81 & 11,80 & 2,34 & 33,48 & 5,98 & 35,83 & 5,43 \\
\hline 5 & 8,73 & 1,93 & 7,73 & 1,47 & 10,60 & 2,88 & 10,71 & 2,45 & 33,45 & 6,05 & 39,17 & 6,49 \\
\hline 6 & 9,36 & 1,78 & 9,49 & 2,89 & 9,71 & 2,52 & 9,58 & 1,87 & 27,23 & 4,60 & 40,30 & 6,07 \\
\hline \multicolumn{13}{|l|}{ Score } \\
\hline 7 & $\underline{7,25}$ & $\underline{1,24}$ & 7,91 & 1,72 & 9,61 & 2,41 & 10,82 & 2,26 & 28,57 & 5,03 & 31,71 & 5,09 \\
\hline 8 & 11,00 & 2,46 & 11,75 & 2,66 & 12,21 & 2,45 & 11,37 & 1,80 & 36,90 & 4,74 & 44,03 & 5,65 \\
\hline 9 & 7,34 & $\underline{1,12}$ & 7,69 & 1,46 & $\underline{7,73}$ & $\underline{1,13}$ & 8,08 & 1,26 & 25,00 & 3,70 & 29,05 & 3,69 \\
\hline 10 & $\underline{15,09}$ & $\underline{3,06}$ & 14,58 & 2,71 & $\underline{17,16}$ & $\underline{3,90}$ & 12,98 & 2,20 & 24,54 & 4,06 & 35,65 & 5,19 \\
\hline
\end{tabular}

Fonte: Fernando Carvalheiro Reiser (2019)

Post hoc: $\mathrm{p}<0.05$ em itálico/sublinhado

\subsection{Análise de Vetor Codificado (Vector Coding)}

4.4.1 Análise Agonista-Antagonista por articulação

O índice da articulação do punho (FSD-ED) para análise do vetor codificado, nas respectivas frequências e modas dos tipos de fases de coordenação, de todas as flechas disparadas, do par de músculos Flexor Superficial dos dedos (FSD) - agonista e o antagonista Extensor dos dedos (ED) estão na figura 11. A moda de coordenação na transição do APA5 até APA1 foi de Fase do Extensor dos Dedos (Fase de ED), e no APA1-APC1 foi de Fase de Flexor Superficial dos Dedos (Fase de FSD); a moda da janela total de frequência foi de Fase do Extensor dos Dedos (35\% dos casos totais APA5-APC1). Na tabela 33, está a análise por desempenho, com a moda de fase de 
coordenação sublinhada por APA-APC, e na tabela 34 estão as modas de transição de cada ajuste de maneira resumida.

Figura 11. Análise de Vetor Codificado das frequências, por percentuais, do índice FSD-ED Acoplamento FSD-ED

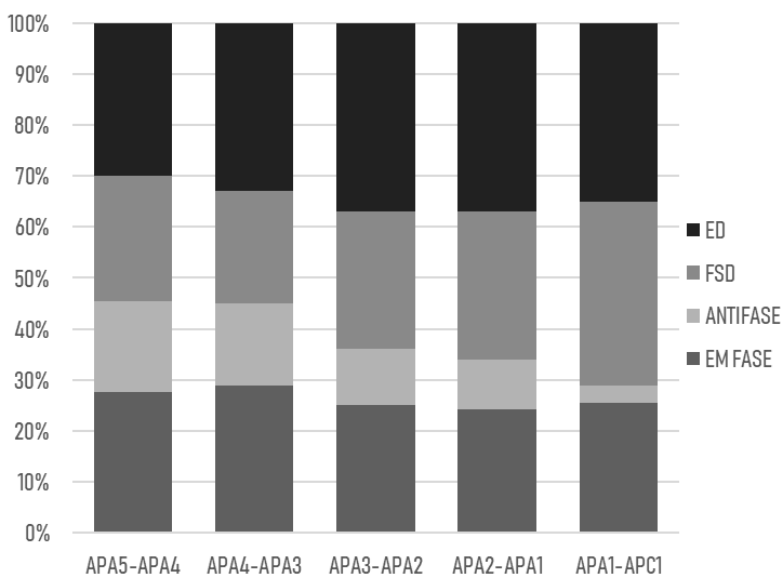

Fonte: Fernando Carvalheiro Reiser (2019)

Tabela 33. Análise de Vetor Codificado - frequências, em percentis, por escore (a moda está sublinhada) Escore

\begin{tabular}{|c|c|c|c|c|c|}
\hline & & 7,00 & 8,00 & $\mathbf{9 , 0 0}$ & 10,00 \\
\hline APA5-APA4 & EM FASE & $29,7 \%$ & $25,2 \%$ & $24,6 \%$ & $34,0 \%$ \\
\hline & ANTIFASE & $18,3 \%$ & $15,5 \%$ & $20,1 \%$ & $15,6 \%$ \\
\hline & FSD & $20,6 \%$ & $28,6 \%$ & $28,0 \%$ & $17,7 \%$ \\
\hline & ED & $31,4 \%$ & $30,6 \%$ & $27,3 \%$ & $32,7 \%$ \\
\hline APA4-APA3 & EM FASE & $22,9 \%$ & $29,1 \%$ & $31,4 \%$ & $30,6 \%$ \\
\hline & ANTIFASE & $14,3 \%$ & $20,4 \%$ & $12,9 \%$ & $18,4 \%$ \\
\hline & FSD & $29,1 \%$ & $20,9 \%$ & $21,2 \%$ & $17,7 \%$ \\
\hline & ED & $33,7 \%$ & $29,6 \%$ & $34,5 \%$ & $33,3 \%$ \\
\hline APA3-APA2 & EM FASE & $23,4 \%$ & $26,2 \%$ & $28,4 \%$ & $19,0 \%$ \\
\hline & ANTIFASE & $13,1 \%$ & $6,8 \%$ & $10,2 \%$ & $16,3 \%$ \\
\hline & FSD & $26,3 \%$ & $34,5 \%$ & $23,5 \%$ & $23,8 \%$ \\
\hline & ED & $37,1 \%$ & $32,5 \%$ & $\underline{37,9 \%}$ & $40,8 \%$ \\
\hline APA2-APA1 & EM FASE & $20,6 \%$ & $23,3 \%$ & $26,5 \%$ & $25,2 \%$ \\
\hline & ANTIFASE & $9,1 \%$ & $12,6 \%$ & $11,0 \%$ & $4,8 \%$ \\
\hline & FSD & $27,4 \%$ & $32,0 \%$ & $23,9 \%$ & $36,7 \%$ \\
\hline & $\overline{\text { ED }}$ & $42,9 \%$ & $\underline{32,0 \%}$ & $\underline{38,6 \%}$ & $33,3 \%$ \\
\hline APA1-APC1 & EM FASE & $25,7 \%$ & $30,1 \%$ & $21,6 \%$ & $25,9 \%$ \\
\hline & ANTIFASE & $3,4 \%$ & $6,3 \%$ & $1,5 \%$ & $2,7 \%$ \\
\hline & FSD & $32,6 \%$ & $\underline{35,9 \%}$ & $37,5 \%$ & $\underline{37,4 \%}$ \\
\hline & ED & $38,3 \%$ & $27,7 \%$ & $39,4 \%$ & $34,0 \%$ \\
\hline
\end{tabular}

Fonte: Fernando Carvalheiro Reiser (2019)

Tabela 34. Moda da fase de coordenação por escore - resumida

\begin{tabular}{cccccc}
\hline & APA5-APA4 & APA4-APA3 & APA3-APA2 & APA2-APA1 & APA1-APC1 \\
\hline 7 & ED & ED & ED & ED & ED \\
8 & ED & ED & FSD & FSD/ED & FSD \\
9 & ED & ED & ED & ED & ED \\
10 & EM FASE & ED & ED & FSD & FSD \\
\hline
\end{tabular}

Fonte: Fernando Carvalheiro Reiser (2019) 
O índice da articulação do cotovelo (BBL-TBL) para o Vetor Codificado, nas respectivas frequências dos tipos de fases de coordenação (FIGURA 12). A moda de coordenação do APA5-APC1 foi de Fase do Tríceps Braquial (36\% dos casos). Na tabela 35 estao os escores, com a moda de fase de coordenação destacada por APAAPC, e na tabela 36 estão as modas de cada ajuste de maneira resumida.

Figura 12. Análise de Vetor Codificado das frequências, por percentuais, do índice BBL-TBL. Acoplamento BBL-TBL

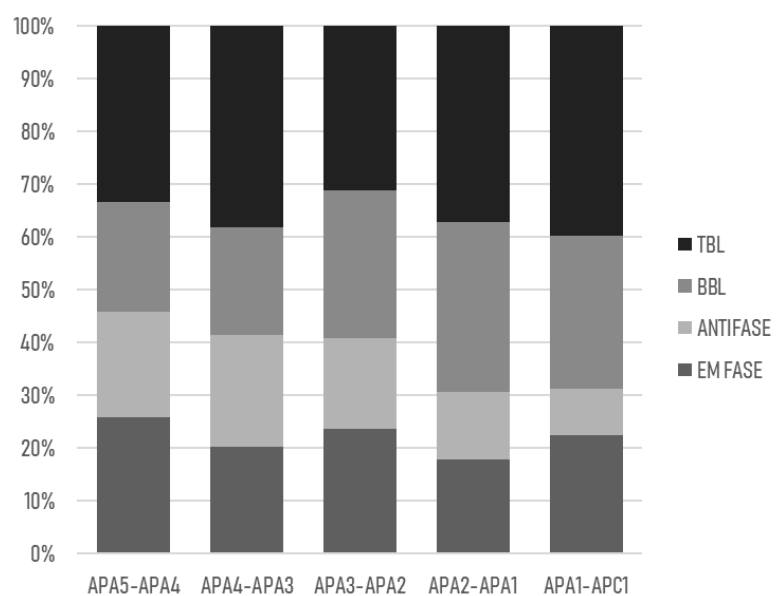

Fonte: Fernando Carvalheiro Reiser (2019)

Tabela 35. Análise de Vetor Codificado - frequências, em percentis, por escore (a moda está sublinhada)

\begin{tabular}{|c|c|c|c|c|c|}
\hline & & \multicolumn{4}{|c|}{ Escore } \\
\hline & & 7,00 & 8,00 & 9,00 & 10,00 \\
\hline \multirow{4}{*}{ APA5-APA4 } & EM FASE & $\underline{28,6 \%}$ & $18,0 \%$ & $27,7 \%$ & $29,9 \%$ \\
\hline & ANTIFASE & $20,0 \%$ & $23,3 \%$ & $20,1 \%$ & $15,6 \%$ \\
\hline & BBL & $27,4 \%$ & $22,8 \%$ & $18,6 \%$ & $14,3 \%$ \\
\hline & TBL & $24,0 \%$ & $\underline{35,9 \%}$ & $\underline{33,7 \%}$ & $\underline{40,1 \%}$ \\
\hline \multirow[t]{4}{*}{ APA4-APA3 } & EM FASE & $25,1 \%$ & $22,8 \%$ & $15,5 \%$ & $19,0 \%$ \\
\hline & ANTIFASE & $18,9 \%$ & $19,9 \%$ & $22,0 \%$ & $24,5 \%$ \\
\hline & BBL & $24,6 \%$ & $22,3 \%$ & $19,3 \%$ & $15,0 \%$ \\
\hline & TBL & $\underline{31,4 \%}$ & $\underline{35,0 \%}$ & $43,2 \%$ & $41,5 \%$ \\
\hline \multirow[t]{4}{*}{ APA3-APA2 } & EM FASE & $\underline{29,7 \%}$ & $25,7 \%$ & $20,5 \%$ & $19,0 \%$ \\
\hline & ANTIFASE & $17,7 \%$ & $18,4 \%$ & $17,4 \%$ & $14,3 \%$ \\
\hline & BBL & $25,7 \%$ & $\underline{28,2 \%}$ & $29,2 \%$ & $29,3 \%$ \\
\hline & TBL & $26,9 \%$ & $27,7 \%$ & $\underline{33,0 \%}$ & $\underline{37,4 \%}$ \\
\hline \multirow[t]{4}{*}{ APA2-APA1 } & EM FASE & $20,0 \%$ & $27,2 \%$ & $15,5 \%$ & $6,1 \%$ \\
\hline & ANTIFASE & $18,3 \%$ & $11,7 \%$ & $11,0 \%$ & $11,6 \%$ \\
\hline & BBL & $\underline{32,6 \%}$ & $\underline{32,5 \%}$ & $32,6 \%$ & $30,6 \%$ \\
\hline & TBL & $29,1 \%$ & $28,6 \%$ & $40,9 \%$ & $\underline{51,7 \%}$ \\
\hline \multirow[t]{4}{*}{ APA1-APC1 } & EM FASE & $27,4 \%$ & $18,9 \%$ & $26,1 \%$ & $14,3 \%$ \\
\hline & ANTIFASE & $5,7 \%$ & $17,5 \%$ & $5,7 \%$ & $6,8 \%$ \\
\hline & BBL & $\underline{33,7 \%}$ & $20,9 \%$ & $30,3 \%$ & $32,0 \%$ \\
\hline & TBL & $33,1 \%$ & $\underline{42,7 \%}$ & $37,9 \%$ & $\underline{46,9 \%}$ \\
\hline
\end{tabular}

Fonte: Fernando Carvalheiro Reiser (2019) 
Tabela 36. Moda da fase de coordenação por escore

\begin{tabular}{cccccc}
\hline & APA5-APA4 & APA4-APA3 & APA3-APA2 & APA2-APA1 & APA1-APC1 \\
\hline 7 & EM FASE & TBL & EM FASE & BBL & BBL \\
8 & TBL & TBL & BBL & BBL & TBL \\
9 & TBL & TBL & TBL & TBL & TBL \\
10 & TBL & TBL & TBL & TBL & TBL \\
\hline
\end{tabular}

Fonte: Fernando Carvalheiro Reiser (2019)

O índice da articulação do ombro (GD-PMC) para análise do vetor codificado, nas respectivas frequências e modas dos tipos de fases de coordenação, de todas as flechas disparadas, do par de músculos Grande Dorsal (GD) - agonista e o antagonista Peitoral Maior - cabeça Clavicular (PMC) estão na figura 13. A moda de coordenação na transição do APA5-APA4 e APA4-APA3 foi de Fase do Grande Dorsal, a moda da transição do APA3 até o APC1 foi de Fase do Peitoral Maior Clavicular. A totalidade dos casos foi de Fase do Peitoral Maior (46\% dos casos totais - APA5-APC1). Na tabela 37 está a análise por desempenho, com a moda de fase de coordenação destacada por APA-APC, e na tabela 38 estão as modas de transição de cada ajuste.

Figura 13. Análise de Vetor Codificado das frequências, por percentuais, do índice GD-PMC.

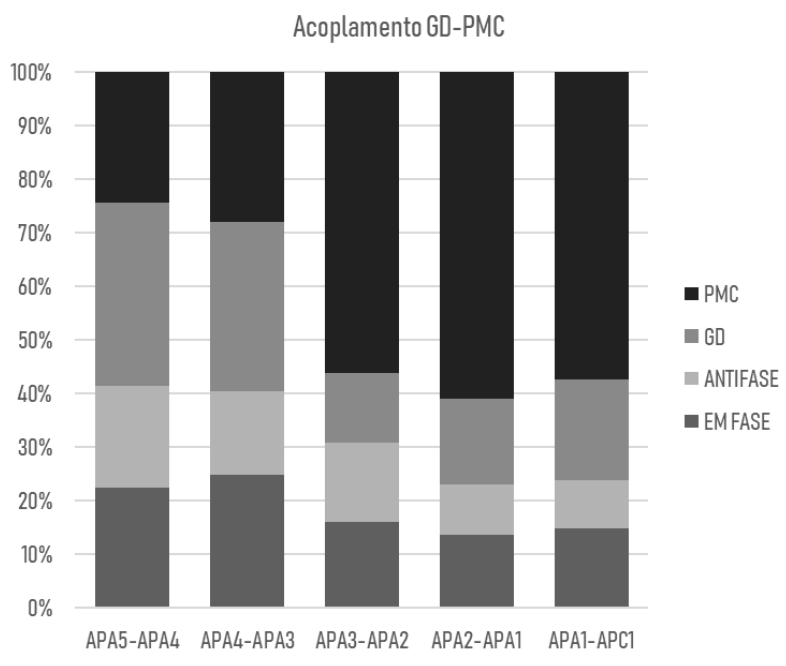

Fonte: Fernando Carvalheiro Reiser (2019) 
Tabela 37. Análise de Vetor Codificado - frequências, em percentis, por escore (a moda está sublinhada)

\begin{tabular}{|c|c|c|c|c|c|}
\hline & & \multicolumn{4}{|c|}{ Escore } \\
\hline & & $\mathbf{7 , 0 0}$ & 8,00 & $\mathbf{9 , 0 0}$ & 10,00 \\
\hline \multirow[t]{4}{*}{ APA5-APA4 } & EM FASE & $20,0 \%$ & $24,3 \%$ & $21,6 \%$ & $24,5 \%$ \\
\hline & ANTIFASE & $22,3 \%$ & $23,8 \%$ & $16,3 \%$ & $13,6 \%$ \\
\hline & GD & $\underline{34,9 \%}$ & $\underline{30,6 \%}$ & $\underline{36,0 \%}$ & $\underline{35,4 \%}$ \\
\hline & PMC & $22,9 \%$ & $21,4 \%$ & $26,1 \%$ & $26,5 \%$ \\
\hline \multirow[t]{4}{*}{ APA4-APA3 } & EM FASE & $21,1 \%$ & $25,7 \%$ & $23,1 \%$ & $\underline{31,5 \%}$ \\
\hline & ANTIFASE & $16,0 \%$ & $16,0 \%$ & $16,7 \%$ & $12,3 \%$ \\
\hline & GD & $\underline{36,6 \%}$ & $27,7 \%$ & $\underline{34,1 \%}$ & $26,7 \%$ \\
\hline & PMC & $26,3 \%$ & $\underline{30,6 \%}$ & $26,1 \%$ & $29,5 \%$ \\
\hline \multirow[t]{4}{*}{ APA3-APA2 } & EM FASE & $17,1 \%$ & $13,1 \%$ & $19,7 \%$ & $12,2 \%$ \\
\hline & ANTIFASE & $12,0 \%$ & $20,4 \%$ & $13,3 \%$ & $12,9 \%$ \\
\hline & GD & $20,0 \%$ & $13,1 \%$ & $7,6 \%$ & $14,3 \%$ \\
\hline & PMC & $\underline{50,9 \%}$ & $\underline{53,4 \%}$ & $\underline{59,5 \%}$ & $\underline{60,5 \%}$ \\
\hline \multirow[t]{4}{*}{ APA2-APA1 } & EM FASE & $17,7 \%$ & $10,7 \%$ & $13,6 \%$ & $12,9 \%$ \\
\hline & ANTIFASE & $10,9 \%$ & $10,2 \%$ & $8,7 \%$ & $7,5 \%$ \\
\hline & GD & $15,4 \%$ & $21,4 \%$ & $14,0 \%$ & $12,9 \%$ \\
\hline & PMC & $\underline{56,0 \%}$ & $\underline{57,8 \%}$ & $\underline{63,6 \%}$ & $\underline{66,7 \%}$ \\
\hline \multirow[t]{4}{*}{ APA1-APC1 } & EM FASE & $18,3 \%$ & $17,5 \%$ & $13,3 \%$ & $10,2 \%$ \\
\hline & ANTIFASE & $11,4 \%$ & $5,8 \%$ & $9,1 \%$ & $10,2 \%$ \\
\hline & GD & $20,6 \%$ & $22,8 \%$ & $14,8 \%$ & $18,4 \%$ \\
\hline & PMC & $49,7 \%$ & $\underline{53,9 \%}$ & $\underline{62,9 \%}$ & $\underline{61,2 \%}$ \\
\hline
\end{tabular}

Fonte: Fernando Carvalheiro Reiser (2019)

Tabela 38. Moda da fase de coordenação por escore

\begin{tabular}{cccccc}
\hline & APA5-APA4 & APA4-APA3 & APA3-APA2 & APA2-APA1 & APA1-APC1 \\
\hline 7 & GD & GD & PMC & PMC & PMC \\
8 & GD & PMC & PMC & PMC & PMC \\
9 & GD & GD & PMC & PMC & PMC \\
10 & GD & EM FASE & PMC & PMC & PMC \\
\hline
\end{tabular}

Fonte: Fernando Carvalheiro Reiser (2019)

O índice da articulação da escápula (TS-SAD) para análise do vetor codificado, nas respectivas frequências e modas dos tipos de fases de coordenação, de todas as flechas disparadas, do par de músculos Trapézio Superior (TS) - agonista e o antagonista Serrátil Anterior - lado dominante (SAD) estão na figura 14. A moda de coordenação na transição do APA5 até o APC1 foi de Fase do Trapézio Superior. A totalidade dos casos foi de Trapézio Superior (42\% dos casos totais - APA5-APC1). Na tabela 39 está a análise por desempenho, com a moda de fase de coordenação destacada por APA-APC, e na tabela 40 estão as modas de transição de cada ajuste. 
Figura 14. Análise de Vetor Codificado das frequências, por percentuais, do índice TS-SAD.

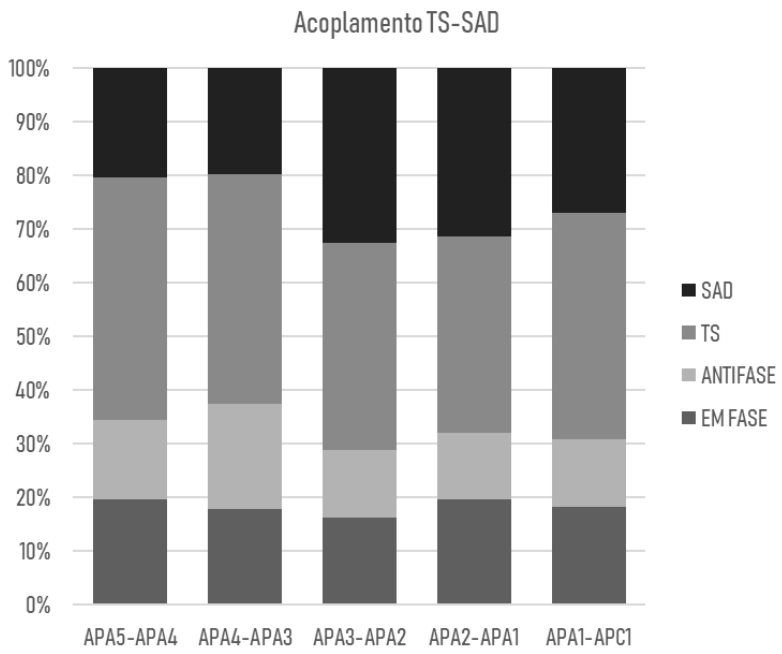

Fonte: Fernando Carvalheiro Reiser (2019)

A moda de coordenação na transição do APA5 até o APC1 foi de Fase do Trapézio Superior. A totalidade dos casos foi de Trapézio Superior (42\% dos casos totais - APA5-APC1). Na tabela 39 está a análise por desempenho, com a moda de fase de coordenação destacada por APA-APC, e na tabela 40 estão as modas de transição de cada ajuste de maneira resumida.

Tabela 39. Análise de Vetor Codificado - frequências, em percentis, por escore (a moda está sublinhada)

Escore

\begin{tabular}{|c|c|c|c|c|c|}
\hline & & 7,00 & 8,00 & 9,00 & 10,00 \\
\hline \multirow[t]{4}{*}{ APA5-APA4 } & EM FASE & $18,9 \%$ & $21,8 \%$ & $20,8 \%$ & $15,6 \%$ \\
\hline & ANTIFASE & $15,4 \%$ & $18,9 \%$ & $14,0 \%$ & $9,5 \%$ \\
\hline & TS & $41,1 \%$ & $42,7 \%$ & $\underline{47,3 \%}$ & $49,7 \%$ \\
\hline & SAD & $24,6 \%$ & $16,5 \%$ & $17,8 \%$ & $25,2 \%$ \\
\hline \multirow[t]{4}{*}{ APA4-APA3 } & EM FASE & $17,7 \%$ & $21,8 \%$ & $16,3 \%$ & $15,6 \%$ \\
\hline & ANTIFASE & $18,3 \%$ & $20,9 \%$ & $23,5 \%$ & $12,2 \%$ \\
\hline & $\underline{\text { TS }}$ & $\underline{41,1 \%}$ & $\underline{38,8 \%}$ & $\underline{46,6 \%}$ & $\underline{43,5 \%}$ \\
\hline & SAD & $22,9 \%$ & $18,4 \%$ & $13,6 \%$ & $28,6 \%$ \\
\hline \multirow[t]{4}{*}{ APA3-APA2 } & $\underline{\text { EM FASE }}$ & $20,6 \%$ & $14,1 \%$ & $15,5 \%$ & $15,0 \%$ \\
\hline & ANTIFASE & $14,3 \%$ & $9,7 \%$ & $11,0 \%$ & $18,4 \%$ \\
\hline & $\underline{\text { TS }}$ & $\underline{43,4 \%}$ & $\underline{46,1 \%}$ & $34,8 \%$ & $28,6 \%$ \\
\hline & SAD & $21,7 \%$ & $30,1 \%$ & $\underline{38,6 \%}$ & $\underline{38,1 \%}$ \\
\hline \multirow[t]{4}{*}{ APA2-APA1 } & EM FASE & $20,0 \%$ & $22,8 \%$ & $18,2 \%$ & $17,7 \%$ \\
\hline & ANTIFASE & $12,0 \%$ & $20,9 \%$ & $7,2 \%$ & $10,2 \%$ \\
\hline & TS & $\underline{43,4 \%}$ & $\underline{31,6 \%}$ & $\underline{38,3 \%}$ & $32,0 \%$ \\
\hline & SAD & $24,6 \%$ & $24,8 \%$ & $36,4 \%$ & $40,1 \%$ \\
\hline \multirow[t]{4}{*}{ APA1-APC1 } & EM FASE & $21,1 \%$ & $20,4 \%$ & $14,8 \%$ & $18,4 \%$ \\
\hline & ANTIFASE & $13,7 \%$ & $7,8 \%$ & $14,4 \%$ & $14,3 \%$ \\
\hline & TS & $\underline{41,1 \%}$ & $\underline{49,0 \%}$ & $\underline{43,6 \%}$ & $31,3 \%$ \\
\hline & SAD & $24,0 \%$ & $22,8 \%$ & $27,3 \%$ & $\underline{36,1 \%}$ \\
\hline
\end{tabular}

Fonte: Fernando Carvalheiro Reiser (2019) 
Tabela 40. Moda da fase de coordenação por escore

\begin{tabular}{cccccc}
\hline & APA5-APA4 & APA4-APA3 & APA3-APA2 & APA2-APA1 & APA1-APC1 \\
\hline 7 & TPS & TPS & TPS & TPS & TPS \\
8 & TPS & TPS & TPS & TPS & TPS \\
9 & TPS & TPS & SAD & TPS & TPS \\
10 & TPS & TPS & SAD & SAD & SAD \\
\hline
\end{tabular}

Fonte: Fernando Carvalheiro Reiser (2019)

O índice da articulação do tronco (RA-ML) para análise do vetor codificado, nas respectivas frequências e modas dos tipos de fases de coordenação, de todas as flechas disparadas, do par de músculos Reto Abdominal (RA) - agonista e o antagonista Multífido Lombar (ML) estão na figura 15. A moda de coordenação na transição do APA5 até o APC1 foi de Fase do Reto Abdominal. A totalidade dos casos foi de Fase do Reto Abdominal (47\% dos casos totais - APA5-APC1). Na tabela 41, está a análise por desempenho, com a moda de fase de coordenação destacada por APA-APC, e na tabela 42 estão as modas de transição de cada ajuste de maneira resumida.

Figura 15. Análise de Vetor Codificado das frequências, por percentuais, do índice RA-ML.

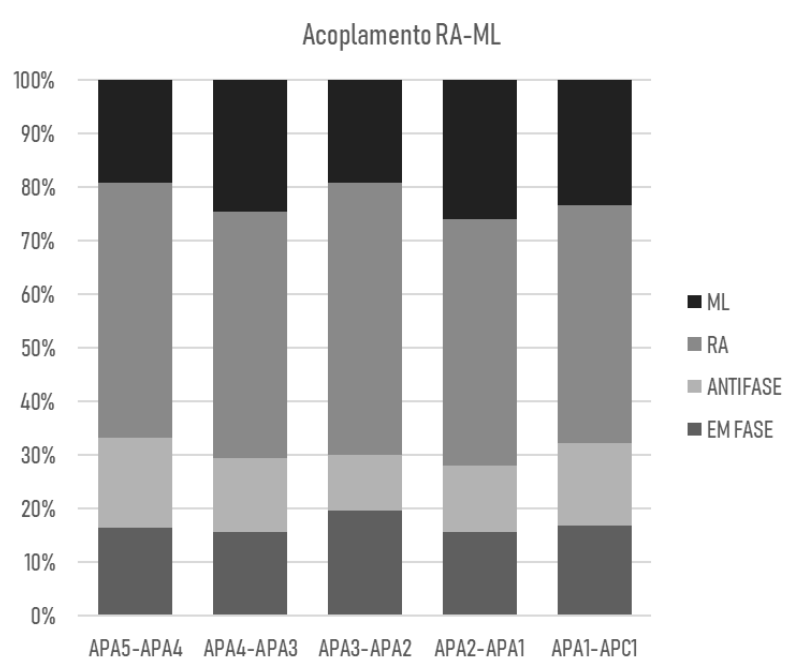

Fonte: Fernando Carvalheiro Reiser (2019) 
Tabela 41. Análise de Vetor Codificado - frequências, em percentis, por escore (a moda está sublinhada)

\begin{tabular}{|c|c|c|c|c|c|}
\hline & & \multicolumn{4}{|c|}{ Escore } \\
\hline & & 7,00 & 8,00 & 9,00 & 10,00 \\
\hline \multirow[t]{4}{*}{ APA5-APA4 } & EM FASE & $14,9 \%$ & $18,9 \%$ & $15,9 \%$ & $15,6 \%$ \\
\hline & ANTIFASE & $16,6 \%$ & $18,9 \%$ & $17,4 \%$ & $13,6 \%$ \\
\hline & $\mathbf{R A}$ & $\underline{56,0 \%}$ & $\underline{43,7 \%}$ & $\underline{46,2 \%}$ & $\underline{45,6 \%}$ \\
\hline & ML & $12,6 \%$ & $18,4 \%$ & $20,5 \%$ & $25,2 \%$ \\
\hline \multirow[t]{4}{*}{ APA4-APA3 } & EM FASE & $16,6 \%$ & $16,5 \%$ & $16,7 \%$ & $11,6 \%$ \\
\hline & ANTIFASE & $13,7 \%$ & $12,6 \%$ & $9,1 \%$ & $24,5 \%$ \\
\hline & $\mathbf{R A}$ & $\underline{54,9 \%}$ & $\underline{40,8 \%}$ & $\underline{46,6 \%}$ & $\underline{41,5 \%}$ \\
\hline & ML & $14,9 \%$ & $30,1 \%$ & $27,7 \%$ & $22,4 \%$ \\
\hline \multirow[t]{4}{*}{ APA3-APA2 } & EM FASE & $21,1 \%$ & $18,0 \%$ & $18,6 \%$ & $21,8 \%$ \\
\hline & ANTIFASE & $8,6 \%$ & $10,7 \%$ & $12,1 \%$ & $9,5 \%$ \\
\hline & $\mathbf{R A}$ & $\underline{60,0 \%}$ & $\underline{47,6 \%}$ & $\underline{52,7 \%}$ & $\underline{41,5 \%}$ \\
\hline & ML & $10,3 \%$ & $23,8 \%$ & $16,7 \%$ & $27,2 \%$ \\
\hline \multirow[t]{4}{*}{ APA2-APA1 } & EM FASE & $15,4 \%$ & $16,0 \%$ & $14,8 \%$ & $17,0 \%$ \\
\hline & ANTIFASE & $18,3 \%$ & $13,1 \%$ & $8,7 \%$ & $10,9 \%$ \\
\hline & $\mathbf{R A}$ & $\underline{49,1 \%}$ & $\underline{45,6 \%}$ & $\underline{47,0 \%}$ & $\underline{41,5 \%}$ \\
\hline & ML & $17,1 \%$ & $25,2 \%$ & $29,5 \%$ & $30,6 \%$ \\
\hline \multirow[t]{4}{*}{ APA1-APC1 } & EM FASE & $13,1 \%$ & $16,0 \%$ & $18,6 \%$ & $19,7 \%$ \\
\hline & ANTIFASE & $13,1 \%$ & $14,6 \%$ & $15,5 \%$ & $18,4 \%$ \\
\hline & $\mathbf{R A}$ & $\underline{50,3 \%}$ & $\underline{42,2 \%}$ & $\underline{44,7 \%}$ & $\underline{40,1 \%}$ \\
\hline & ML & $23,4 \%$ & $27,2 \%$ & $21,2 \%$ & $21,8 \%$ \\
\hline
\end{tabular}

Fonte: Fernando Carvalheiro Reiser (2019)

Tabela 42. Moda da fase de coordenação por escore

\begin{tabular}{cccccc}
\hline & APA5-APA4 & APA4-APA3 & APA3-APA2 & APA2-APA1 & APA1-APC1 \\
\hline 7 & RA & RA & RA & RA & RA \\
8 & RA & RA & RA & RA & RA \\
9 & RA & RA & RA & RA & RA \\
10 & RA & RA & RA & RA & RA \\
\hline
\end{tabular}

Fonte: Fernando Carvalheiro Reiser (2019) 


\subsubsection{Distal-Proximal (Punho e cotovelo)}

O índice de agonistas (distal-proximal) para as articulações do punho e do cotovelo (FSD-BBL) para análise do vetor codificado, nas respectivas frequências e modas dos tipos de fases de coordenação, de todas as flechas disparadas, do par de músculos FSD - agonista do punho e o agonista do cotovelo BBL estão na figura 16. A moda de coordenação na transição do APA5-APA4, APA4-APA3 e APA3-APA2 foi de Fase do Bíceps Braquial; na transição APA2-APA1 e APA1-APC1 foi de Fase do Flexor Superficial dos Dedos. A totalidade dos casos foi de Fase do Flexor Superficial dos Dedos (29\% dos casos totais - APA5-APC1). Na tabela 43 está a análise por desempenho, com a moda de fase de coordenação destacada por APA-APC, e na tabela 44 estão as modas de transição de cada ajuste de maneira resumida.

Figura 16. Análise de Vetor Codificado das frequências, por percentuais, do índice FSD-BBL.

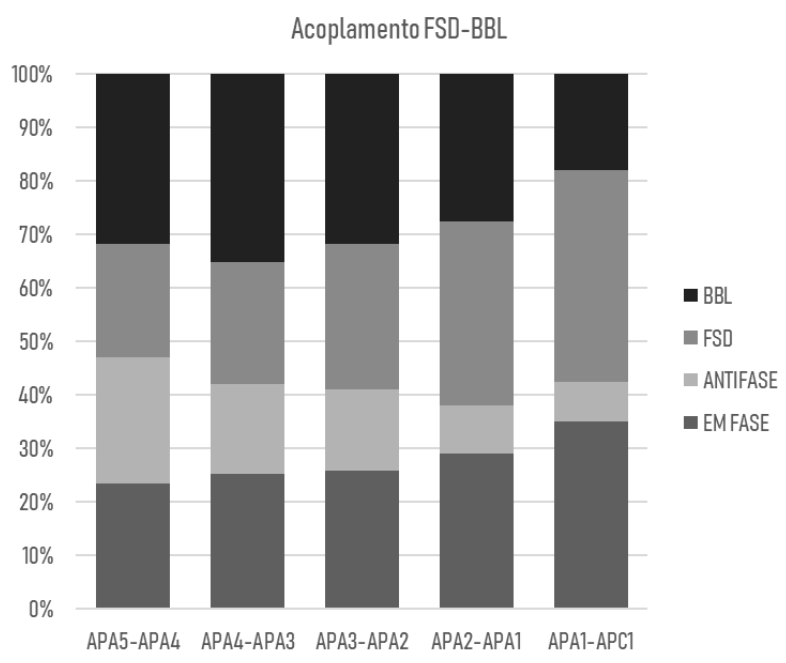

Fonte: Fernando Carvalheiro Reiser (2019) 
Tabela 43. Análise de Vetor Codificado - frequências, em percentis, por escore (a moda está sublinhada).

\begin{tabular}{|c|c|c|c|c|c|}
\hline & & \multicolumn{4}{|c|}{ Escore } \\
\hline & & 7,00 & 8,00 & 9,00 & 10,00 \\
\hline \multirow[t]{4}{*}{ APA5-APA4 } & EM FASE & $25,1 \%$ & $20,4 \%$ & $24,6 \%$ & $23,1 \%$ \\
\hline & ANTIFASE & $20,6 \%$ & $26,7 \%$ & $22,3 \%$ & $25,2 \%$ \\
\hline & FSD & $17,1 \%$ & $18,4 \%$ & $25,4 \%$ & $23,1 \%$ \\
\hline & BBL & $37,1 \%$ & $34,5 \%$ & $27,7 \%$ & $\underline{28,6 \%}$ \\
\hline \multirow[t]{4}{*}{ APA4-APA3 } & EM FASE & $24,0 \%$ & $27,7 \%$ & $25,8 \%$ & $22,4 \%$ \\
\hline & ANTIFASE & $18,9 \%$ & $18,0 \%$ & $15,9 \%$ & $14,3 \%$ \\
\hline & FSD & $18,9 \%$ & $20,9 \%$ & $26,5 \%$ & $23,1 \%$ \\
\hline & BBL & $\underline{38,3 \%}$ & $\underline{33,5 \%}$ & $\underline{31,8 \%}$ & $\underline{40,1 \%}$ \\
\hline \multirow[t]{4}{*}{ APA3-APA2 } & EM FASE & $\underline{30,9 \%}$ & $\underline{28,2 \%}$ & $25,4 \%$ & $17,7 \%$ \\
\hline & ANTIFASE & $11,4 \%$ & $17,0 \%$ & $18,2 \%$ & $11,6 \%$ \\
\hline & FSD & $28,0 \%$ & $27,7 \%$ & $27,7 \%$ & $24,5 \%$ \\
\hline & BBL & $29,7 \%$ & $27,2 \%$ & $\underline{28,8 \%}$ & $46,3 \%$ \\
\hline \multirow[t]{4}{*}{ APA2-APA1 } & EM FASE & $28,0 \%$ & $30,6 \%$ & $28,0 \%$ & $29,9 \%$ \\
\hline & ANTIFASE & $9,7 \%$ & $9,7 \%$ & $10,2 \%$ & $5,4 \%$ \\
\hline & FSD & $\underline{36,0 \%}$ & $\underline{32,0 \%}$ & $\underline{33,0 \%}$ & $\underline{38,1 \%}$ \\
\hline & BBL & $26,3 \%$ & $27,7 \%$ & $28,8 \%$ & $26,5 \%$ \\
\hline \multirow[t]{4}{*}{ APA1-APC1 } & EM FASE & $32,6 \%$ & $35,9 \%$ & $\underline{38,6 \%}$ & $29,9 \%$ \\
\hline & ANTIFASE & $7,4 \%$ & $5,8 \%$ & $8,0 \%$ & $8,8 \%$ \\
\hline & FSD & $\underline{41,7 \%}$ & $\underline{40,3 \%}$ & $\underline{38,6 \%}$ & $\underline{38,1 \%}$ \\
\hline & BBL & $18,3 \%$ & $18,0 \%$ & $14,8 \%$ & $23,1 \%$ \\
\hline
\end{tabular}

Fonte: Fernando Carvalheiro Reiser (2019)

Tabela 44. Moda da fase de coordenação por escore

\begin{tabular}{cccccc}
\hline & APA5-APA4 & APA4-APA3 & APA3-APA2 & APA2-APA1 & APA1-APC1 \\
\hline 7 & BBL & BBL & EM FASE & FSD & FSD \\
8 & BBL & BBL & FSD & FSD & FSD \\
9 & BBL & BBL & BBL & FSD & FSD/EM \\
10 & BBL & BBL & BBL & FSD & FSD \\
\hline
\end{tabular}

Fonte: Fernando Carvalheiro Reiser (2019)

O índice de agonista-antagonista (distal-proximal) para as articulações do punho e do cotovelo (FSD-TBL) para análise do vetor codificado, nas respectivas frequências e modas dos tipos de fases de coordenação, de todas as flechas disparadas, do par de músculos FSD - agonista do punho e o antagonista do cotovelo TBL estão na figura 17. A moda de coordenação na transição do APA5-APA4, APA4-APA3 e APA3-APA2 foi de Fase do Flexor Superficial dos Dedos; na transição APA2-APA1 e APA1-APC1 foi de Fase do Tríceps Braquial. A totalidade dos casos foi de Fase do Tríceps Braquial 
(35\% dos casos totais - APA5-APC1). Na tabela 45 está a análise por desempenho, com a moda de fase de coordenação destacada por APA-APC, e na tabela 46 estão as modas de transição de cada ajuste de maneira resumida.

Figura 17. Análise de Vetor Codificado das frequências, por percentuais, do índice FSD-TBL.

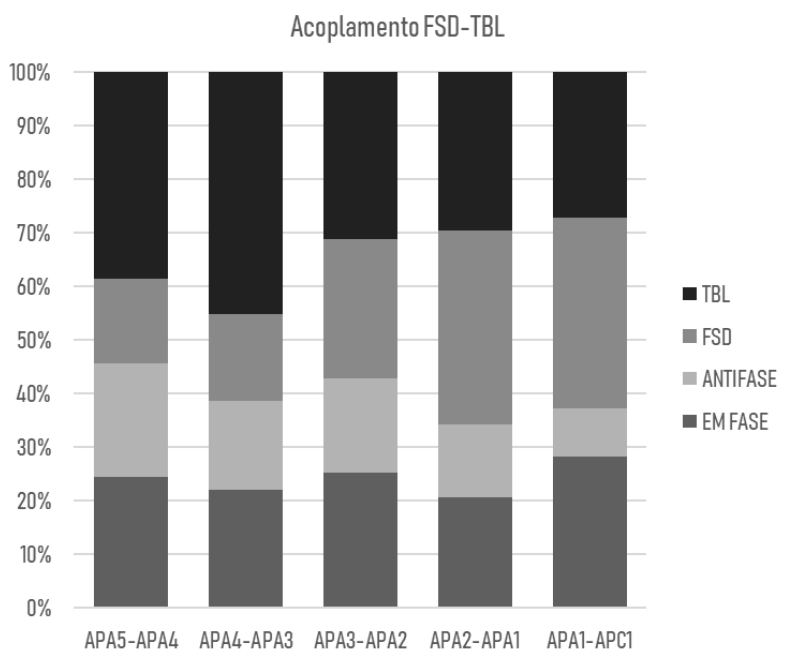

Fonte: Fernando Carvalheiro Reiser (2019)

Tabela 45. Análise de Vetor Codificado - frequências, em percentis, por escore (a moda está sublinhada).

\begin{tabular}{|c|c|c|c|c|c|}
\hline & & \multicolumn{4}{|c|}{ Escore } \\
\hline & & $\mathbf{7 , 0 0}$ & $\mathbf{8 , 0 0}$ & 9,00 & 10,00 \\
\hline \multirow[t]{4}{*}{ APA5-APA4 } & EM FASE & $25,1 \%$ & $21,8 \%$ & $23,9 \%$ & $28,6 \%$ \\
\hline & ANTIFASE & $22,9 \%$ & $20,4 \%$ & $20,8 \%$ & $21,1 \%$ \\
\hline & FSD & $20,6 \%$ & $16,0 \%$ & $14,8 \%$ & $11,6 \%$ \\
\hline & TBL & $\underline{31,4 \%}$ & $\underline{41,7 \%}$ & $\underline{40,5 \%}$ & $\underline{38,8 \%}$ \\
\hline \multirow[t]{4}{*}{ APA4-APA3 } & EM FASE & $24,6 \%$ & $18,4 \%$ & $23,1 \%$ & $22,4 \%$ \\
\hline & ANTIFASE & $12,6 \%$ & $23,8 \%$ & $16,3 \%$ & $11,6 \%$ \\
\hline & FSD & $17,7 \%$ & $19,4 \%$ & $15,2 \%$ & $11,6 \%$ \\
\hline & TBL & $\underline{45,1 \%}$ & $\underline{38,3 \%}$ & $\underline{45,5 \%}$ & $\underline{54,4 \%}$ \\
\hline \multirow[t]{4}{*}{ APA3-APA2 } & EM FASE & $22,3 \%$ & $\underline{30,1 \%}$ & $26,1 \%$ & $20,4 \%$ \\
\hline & ANTIFASE & $17,7 \%$ & $23,3 \%$ & $12,5 \%$ & $19,0 \%$ \\
\hline & FSD & $\underline{30,9 \%}$ & $25,2 \%$ & $26,9 \%$ & $19,7 \%$ \\
\hline & TBL & $29,1 \%$ & $21,4 \%$ & $\underline{34,5 \%}$ & $\underline{40,8 \%}$ \\
\hline \multirow[t]{4}{*}{ APA2-APA1 } & EM FASE & $24,6 \%$ & $24,8 \%$ & $15,9 \%$ & $19,0 \%$ \\
\hline & ANTIFASE & $10,3 \%$ & $13,6 \%$ & $15,9 \%$ & $13,6 \%$ \\
\hline & FSD & $\underline{41,7 \%}$ & $\underline{35,9 \%}$ & $\underline{36,7 \%}$ & $28,6 \%$ \\
\hline & TBL & $23,4 \%$ & $25,7 \%$ & $31,4 \%$ & $\underline{38,8 \%}$ \\
\hline \multirow[t]{4}{*}{ APA1-APC1 } & EM FASE & $33,7 \%$ & $25,2 \%$ & $26,1 \%$ & $29,3 \%$ \\
\hline & ANTIFASE & $8,0 \%$ & $13,6 \%$ & $6,4 \%$ & $8,8 \%$ \\
\hline & FSD & $39,4 \%$ & $28,6 \%$ & $\underline{42,0 \%}$ & $29,3 \%$ \\
\hline & TBL & $18,9 \%$ & $32,5 \%$ & $25,4 \%$ & $32,7 \%$ \\
\hline
\end{tabular}

Fonte: Fernando Carvalheiro Reiser (2019) 
Tabela 46. Moda da fase de coordenação por escore resumida

\begin{tabular}{cccccc}
\hline & APA5-APA4 & APA4-APA3 & APA3-APA2 & APA2-APA1 & APA1-APC1 \\
\hline 7 & TBL & TBL & FSD & FSD & FSD \\
8 & TBL & TBL & EM FASE & FSD & TBL \\
9 & TBL & TBL & TBL & FSD & FSD \\
10 & TBL & TBL & TBL & TBL & TBL \\
\hline
\end{tabular}

Fonte: Fernando Carvalheiro Reiser (2019)

O índice de antagonista-agonista (distal-proximal) para as articulações do punho e do cotovelo (ED-BBL) para análise do vetor codificado, nas respectivas frequências e modas dos tipos de fases de coordenação, de todas as flechas disparadas, do par de músculos ED - agonista do punho e o antagonista do cotovelo BBL estão na figura 18. A moda de coordenação na transição do APA5-APA4 e APA4-APA3 foi de Fase do Bíceps Braquial, e APA3-APA2, APA2-APA1, APA1-APC1 foi de Fase do Extensor dos Dedos. A totalidade dos casos foi de Fase do Extensor dos Dedos (31\% dos casos totais - APA5-APC1). Na tabela 47 está a análise por desempenho, com a moda de fase de coordenação destacada por APA-APC, e na tabela 48 estão as modas de transição de cada ajuste de maneira resumida.

Figura 18. Análise de Vetor Codificado das frequências, por percentuais, do índice ED-BBL.

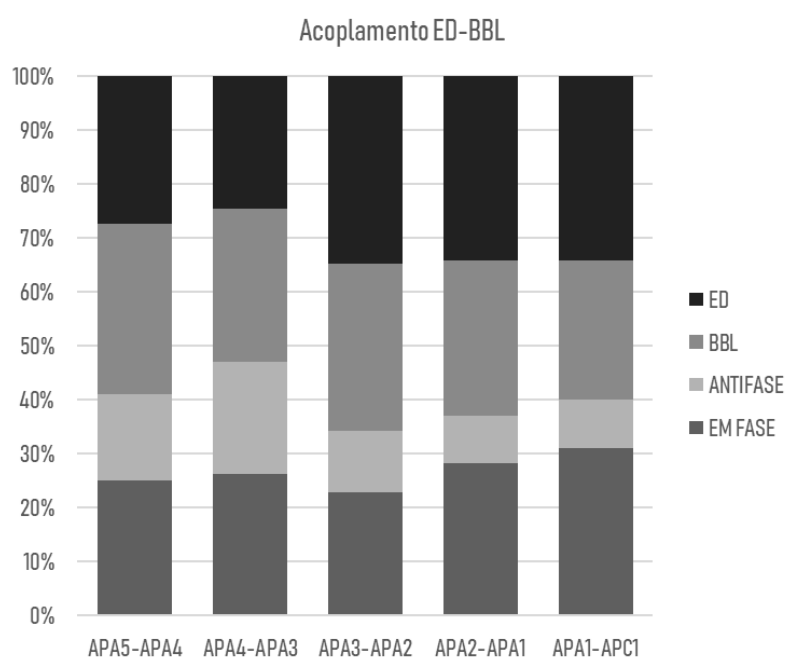

Fonte: Fernando Carvalheiro Reiser (2019) 
Tabela 47. Análise de Vetor Codificado - frequências, em percentis, por escore (a moda está sublinhada).

\begin{tabular}{|c|c|c|c|c|c|}
\hline & & \multicolumn{4}{|c|}{ Escore } \\
\hline & & 7,00 & 8,00 & 9,00 & 10,00 \\
\hline \multirow[t]{4}{*}{ APA5-APA4 } & EM FASE & $25,7 \%$ & $23,8 \%$ & $24,2 \%$ & $27,9 \%$ \\
\hline & ANTIFASE & $17,7 \%$ & $16,5 \%$ & $15,2 \%$ & $14,3 \%$ \\
\hline & BBL & $\underline{32,0 \%}$ & $\underline{35,9 \%}$ & $\underline{30,7 \%}$ & $27,2 \%$ \\
\hline & ED & $24,6 \%$ & $23,8 \%$ & $29,9 \%$ & $\underline{30,6 \%}$ \\
\hline \multirow[t]{4}{*}{ APA4-APA3 } & EM FASE & $17,1 \%$ & $24,8 \%$ & $\underline{32,6 \%}$ & $27,9 \%$ \\
\hline & ANTIFASE & $23,4 \%$ & $24,3 \%$ & $18,2 \%$ & $17,0 \%$ \\
\hline & BBL & $\underline{36,0 \%}$ & $26,2 \%$ & $23,1 \%$ & $\underline{32,0 \%}$ \\
\hline & ED & $23,4 \%$ & $24,8 \%$ & $26,1 \%$ & $23,1 \%$ \\
\hline \multirow[t]{4}{*}{ APA3-APA2 } & EM FASE & $21,1 \%$ & $19,4 \%$ & $26,1 \%$ & $23,8 \%$ \\
\hline & ANTIFASE & $6,9 \%$ & $17,5 \%$ & $12,1 \%$ & $6,8 \%$ \\
\hline & BBL & $29,7 \%$ & $\underline{34,0 \%}$ & $27,3 \%$ & $\underline{35,4 \%}$ \\
\hline & ED & $\underline{42,3 \%}$ & $29,1 \%$ & $\underline{34,5 \%}$ & $34,0 \%$ \\
\hline \multirow[t]{4}{*}{ APA2-APA1 } & EM FASE & $27,4 \%$ & $27,2 \%$ & $26,5 \%$ & $\underline{34,0 \%}$ \\
\hline & ANTIFASE & $6,3 \%$ & $7,3 \%$ & $9,5 \%$ & $12,2 \%$ \\
\hline & BBL & $26,3 \%$ & $\underline{36,9 \%}$ & $24,2 \%$ & $28,6 \%$ \\
\hline & ED & $\underline{40,0 \%}$ & $28,6 \%$ & $\underline{39,8 \%}$ & $25,2 \%$ \\
\hline \multirow[t]{4}{*}{ APA1-APC1 } & EM FASE & $34,3 \%$ & $\underline{33,5 \%}$ & $25,4 \%$ & $34,0 \%$ \\
\hline & ANTIFASE & $4,6 \%$ & $11,7 \%$ & $11,7 \%$ & $5,4 \%$ \\
\hline & BBL & $20,0 \%$ & $28,2 \%$ & $22,7 \%$ & $\underline{35,4 \%}$ \\
\hline & ED & $\underline{41,1 \%}$ & $26,7 \%$ & $\underline{40,2 \%}$ & $25,2 \%$ \\
\hline
\end{tabular}

Fonte: Fernando Carvalheiro Reiser (2019)

Tabela 48. Moda da fase de coordenação por escore

\begin{tabular}{cccccc}
\hline & APA5-APA4 & APA4-APA3 & APA3-APA2 & APA2-APA1 & APA1-APC1 \\
\hline 7 & BBL & BBL & BBL & ED & ED \\
8 & BBL & BBL & BBL & BBL & EM FASE \\
9 & BBL & EM FASE & ED & ED & ED \\
10 & ED & BBL & BBL & EM FASE & BBL \\
\hline
\end{tabular}

Fonte: Fernando Carvalheiro Reiser (2019)

O índice de antagonistas (distal-proximal) para as articulações do punho e do cotovelo (ED-TBL) para análise do vetor codificado, nas respectivas frequências e modas dos tipos de fases de coordenação, de todas as flechas disparadas, do par de músculos ED - antagonista do punho e o antagonista do cotovelo TBL estão na figura 19. A moda de coordenação na transição do APA5-APA4 e APA4-APA3 foi de Fase do Tríceps Braquial - cabeça longa, e APA3-APA2, APA2-APA1, APA1-APC1 foi de Fase do Extensor dos Dedos. A totalidade dos casos foi de Fase do Tríceps Braquial (35\% dos casos totais - APA5-APC1). Na tabela 49, está a análise por desempenho, 
com a moda de fase de coordenação destacada por APA-APC, e na tabela 50 estão as modas de transição de cada ajuste de maneira resumida.

Figura 19. Análise de Vetor Codificado das frequências, por percentuais, do índice ED-TBL.

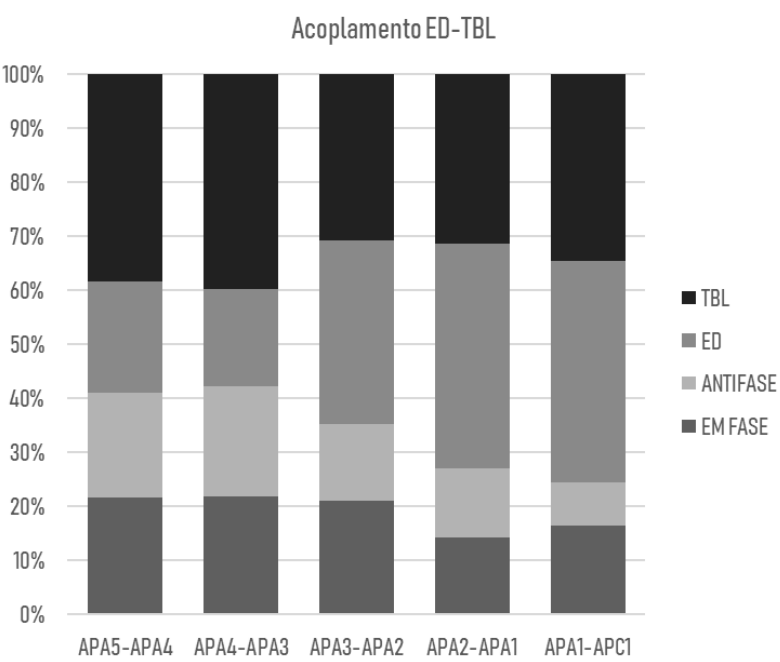

Fonte: Fernando Carvalheiro Reiser (2019)

Tabela 49. Análise de Vetor Codificado - frequências, em percentis, por escore (a moda está sublinhada).

\begin{tabular}{|c|c|c|c|c|c|}
\hline & & \multicolumn{4}{|c|}{ Escore } \\
\hline & & 7,00 & $\mathbf{8 , 0 0}$ & 9,00 & 10,00 \\
\hline \multirow[t]{4}{*}{ APA5-APA4 } & EM FASE & $21,7 \%$ & $18,4 \%$ & $22,7 \%$ & $24,5 \%$ \\
\hline & ANTIFASE & $18,9 \%$ & $21,4 \%$ & $20,1 \%$ & $15,6 \%$ \\
\hline & $\underline{\text { ED }}$ & $28,6 \%$ & $19,9 \%$ & $16,3 \%$ & $19,7 \%$ \\
\hline & TBL & $\underline{30,9 \%}$ & $\underline{40,3 \%}$ & $\underline{40,9 \%}$ & $\underline{40,1 \%}$ \\
\hline \multirow[t]{4}{*}{ APA4-APA3 } & EM FASE & $21,7 \%$ & $21,8 \%$ & $23,1 \%$ & $19,7 \%$ \\
\hline & ANTIFASE & $19,4 \%$ & $18,9 \%$ & $21,6 \%$ & $21,1 \%$ \\
\hline & $\underline{\text { ED }}$ & $21,1 \%$ & $20,4 \%$ & $18,2 \%$ & $11,6 \%$ \\
\hline & TBL & $37,7 \%$ & $38,8 \%$ & $37,1 \%$ & $47,6 \%$ \\
\hline \multirow[t]{4}{*}{ APA3-APA2 } & $\underline{\text { EM FASE }}$ & $20,0 \%$ & $22,3 \%$ & $20,5 \%$ & $21,8 \%$ \\
\hline & ANTIFASE & $14,3 \%$ & $16,5 \%$ & $11,0 \%$ & $16,3 \%$ \\
\hline & ED & $\underline{41,1 \%}$ & $27,7 \%$ & $\underline{36,0 \%}$ & $\underline{31,3 \%}$ \\
\hline & TBL & $24,6 \%$ & $\underline{33,5 \%}$ & $32,6 \%$ & $30,6 \%$ \\
\hline \multirow[t]{4}{*}{ APA2-APA1 } & EM FASE & $17,7 \%$ & $12,6 \%$ & $14,0 \%$ & $12,9 \%$ \\
\hline & ANTIFASE & $10,9 \%$ & $16,5 \%$ & $14,0 \%$ & $8,2 \%$ \\
\hline & $\underline{\text { ED }}$ & $\underline{51,4 \%}$ & $\underline{36,9 \%}$ & $\underline{40,9 \%}$ & $36,7 \%$ \\
\hline & TBL & $20,0 \%$ & $34,0 \%$ & $31,1 \%$ & $\underline{42,2 \%}$ \\
\hline \multirow[t]{4}{*}{ APA1-APC1 } & EM FASE & $17,1 \%$ & $18,4 \%$ & $16,3 \%$ & $12,9 \%$ \\
\hline & ANTIFASE & $5,1 \%$ & $9,2 \%$ & $7,6 \%$ & $10,9 \%$ \\
\hline & $\underline{\text { ED }}$ & $\underline{54,3 \%}$ & $31,6 \%$ & $\underline{42,4 \%}$ & $36,1 \%$ \\
\hline & TBL & $23,4 \%$ & $40,8 \%$ & $33,7 \%$ & $40,1 \%$ \\
\hline
\end{tabular}

Fonte: Fernando Carvalheiro Reiser (2019) 
Tabela 50. Moda da fase de coordenação por escore

Fonte: Fernando Carvalheiro Reiser (2019)

\begin{tabular}{cccccc}
\hline & APA5-APA4 & APA4-APA3 & APA3-APA2 & APA2-APA1 & APA1-APC1 \\
\hline 7 & TBL & TBL & ED & ED & ED \\
8 & TBL & TBL & TBL & ED & TBL \\
9 & TBL & TBL & ED & ED & ED \\
10 & TBL & TBL & ED & TBL & TBL \\
\hline
\end{tabular}

\subsection{3 Índice Glenoumeral-Escápula}

O índice de antagonistas da articulação glenoumeral-escápula (PMCSAD) para análise do vetor codificado, nas respectivas frequências e modas dos tipos de fases de coordenação, de todas as flechas disparadas, do par de músculos PMC - antagonista da glenoumeral e o antagonista da escápula - SAD estão na figura 20. A moda de coordenação na transição do APA5-APA4 e APA4-APA3 foi de Fase do Serrátil Anterior, e APA3-APA2, APA2-APA1, APA1-APC1 foi de Fase do Peitoral Maior Cabeça Clavicular. A totalidade dos casos foi de Fase do Peitoral Maior (36\% dos casos totais - APA5-APC1). Na tabela 51 está a análise por desempenho, com a moda de fase de coordenação destacada por APA-APC, e na tabela 52 estão as modas de transição de cada ajuste de maneira resumida.

Figura 20. Análise de Vetor Codificado das frequências, por percentuais, do índice PMC-SAD.

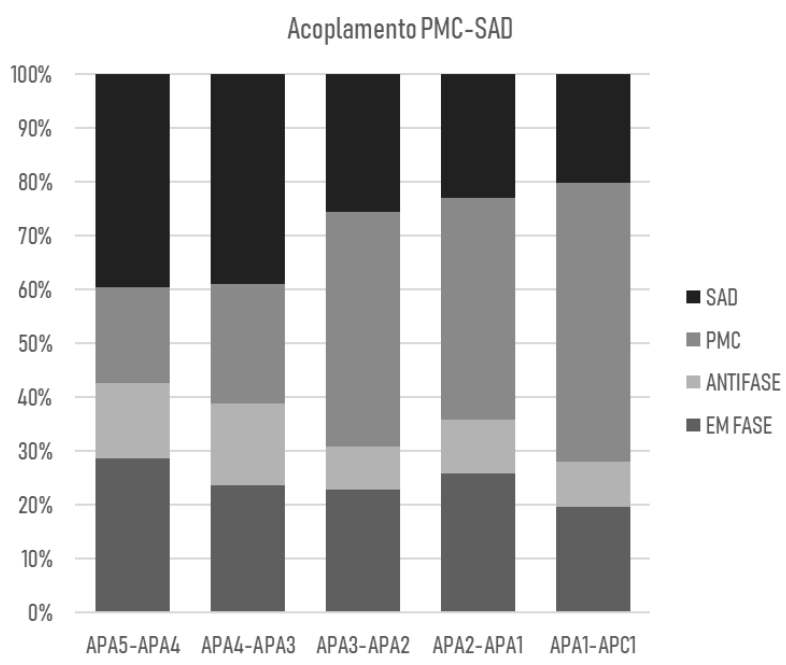

Fonte: Fernando Carvalheiro Reiser (2019) 
Tabela 51. Análise de Vetor Codificado - frequências, em percentis, por escore (a moda está sublinhada).

Escore

\begin{tabular}{|c|c|c|c|c|c|}
\hline & & $\mathbf{7 , 0 0}$ & 8,00 & 9,00 & 10,00 \\
\hline \multirow[t]{4}{*}{ APA5-APA4 } & EM FASE & $22,9 \%$ & $30,6 \%$ & $26,5 \%$ & $\underline{36,7 \%}$ \\
\hline & ANTIFASE & $9,7 \%$ & $11,2 \%$ & $18,9 \%$ & $14,3 \%$ \\
\hline & $\underline{\text { PMC }}$ & $17,1 \%$ & $13,6 \%$ & $20,1 \%$ & $20,4 \%$ \\
\hline & SAD & $\underline{50,3 \%}$ & $\underline{44,7 \%}$ & $34,5 \%$ & $28,6 \%$ \\
\hline \multirow[t]{4}{*}{ APA4-APA3 } & EM FASE & $20,6 \%$ & $24,3 \%$ & $31,1 \%$ & $13,6 \%$ \\
\hline & ANTIFASE & $7,4 \%$ & $22,3 \%$ & $12,5 \%$ & $18,4 \%$ \\
\hline & $\underline{\text { PMC }}$ & $20,6 \%$ & $17,0 \%$ & $21,2 \%$ & $\underline{34,0 \%}$ \\
\hline & SAD & $\underline{51,4 \%}$ & $\underline{36,4 \%}$ & $\underline{35,2 \%}$ & $\underline{34,0 \%}$ \\
\hline \multirow[t]{4}{*}{ APA3-APA2 } & EM FASE & $17,7 \%$ & $22,8 \%$ & $27,3 \%$ & $21,1 \%$ \\
\hline & ANTIFASE & $9,1 \%$ & $9,2 \%$ & $5,7 \%$ & $8,8 \%$ \\
\hline & PMC & $39,4 \%$ & $\underline{46,1 \%}$ & $\underline{40,9 \%}$ & $\underline{50,3 \%}$ \\
\hline & SAD & $33,7 \%$ & $21,8 \%$ & $26,1 \%$ & $19,7 \%$ \\
\hline \multirow[t]{4}{*}{ APA2-APA1 } & EM FASE & $27,4 \%$ & $27,2 \%$ & $17,4 \%$ & $36,7 \%$ \\
\hline & ANTIFASE & $15,4 \%$ & $12,1 \%$ & $5,7 \%$ & $8,8 \%$ \\
\hline & $\underline{\text { PMC }}$ & $\underline{41,1 \%}$ & $\underline{41,7 \%}$ & $\underline{42,4 \%}$ & $\underline{38,1 \%}$ \\
\hline & SAD & $16,0 \%$ & $18,9 \%$ & $34,5 \%$ & $16,3 \%$ \\
\hline \multirow[t]{4}{*}{ APA1-APC1 } & EM FASE & $24,6 \%$ & $17,5 \%$ & $18,2 \%$ & $19,0 \%$ \\
\hline & ANTIFASE & $8,0 \%$ & $12,1 \%$ & $3,4 \%$ & $12,9 \%$ \\
\hline & PMC & $\underline{44,6 \%}$ & $\underline{54,4 \%}$ & $\underline{57,2 \%}$ & $\underline{46,9 \%}$ \\
\hline & SAD & $22,9 \%$ & $16,0 \%$ & $21,2 \%$ & $21,1 \%$ \\
\hline
\end{tabular}

Fonte: Fernando Carvalheiro Reiser (2019)

Tabela 52. Moda da fase de coordenação por escore

\begin{tabular}{cccccc}
\hline & APA5-APA4 & APA4-APA3 & APA3-APA2 & APA2-APA1 & APA1-APC1 \\
\hline 7 & SAD & SAD & PMC & PMC & PMC \\
8 & SAD & SAD & PMC & PMC & PMC \\
9 & SAD & SAD & PMC & PMC & PMC \\
10 & EM FASE & SAD/PMC & PMC & PMC & PMC \\
\hline
\end{tabular}

Fonte: Fernando Carvalheiro Reiser (2019)

O índice de agonistas da articulação glenoumeral-escápula (GD-TS) para análise do vetor codificado, nas respectivas frequências e modas dos tipos de fases de coordenação, de todas as flechas disparadas, do par de músculos GD - agonista da glenoumeral e o agonista da escápula - TS estão na figura 21. A moda de coordenação na transição do APA5 até o APC1 foi de Fase do Trapézio Superior. A totalidade dos casos foi de Fase do Trapézio Superior (51\% dos casos totais - APA5-APC1). Na tabela 53 está a análise por desempenho, com a moda de fase de coordenação destacada por APA-APC, e na tabela 54 estão as modas de transição de cada ajuste. 
Figura 21. Análise de Vetor Codificado das frequências, por percentuais, do índice GD-TS Acoplamento GD-TS

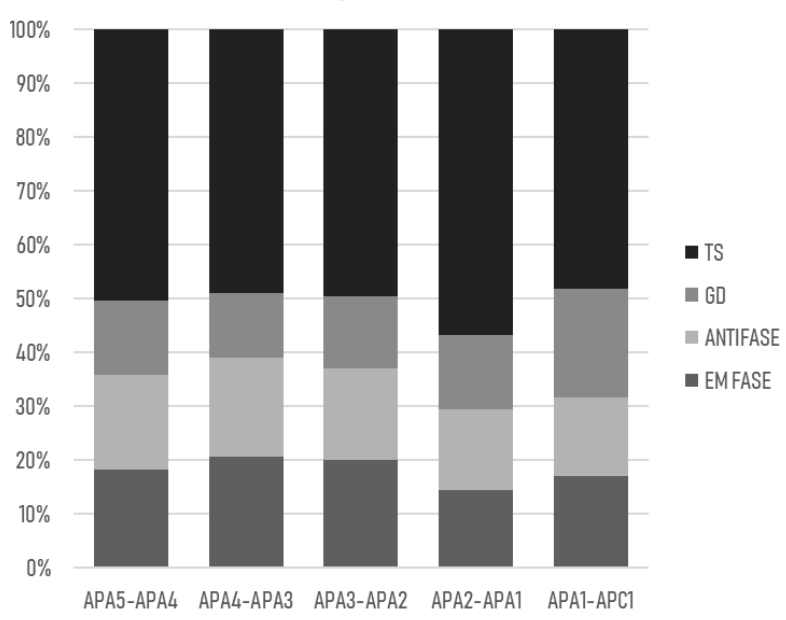

Fonte: Fernando Carvalheiro Reiser (2019)

Tabela 53. Análise de Vetor Codificado - frequências, em percentis, por escore (a moda está sublinhada).

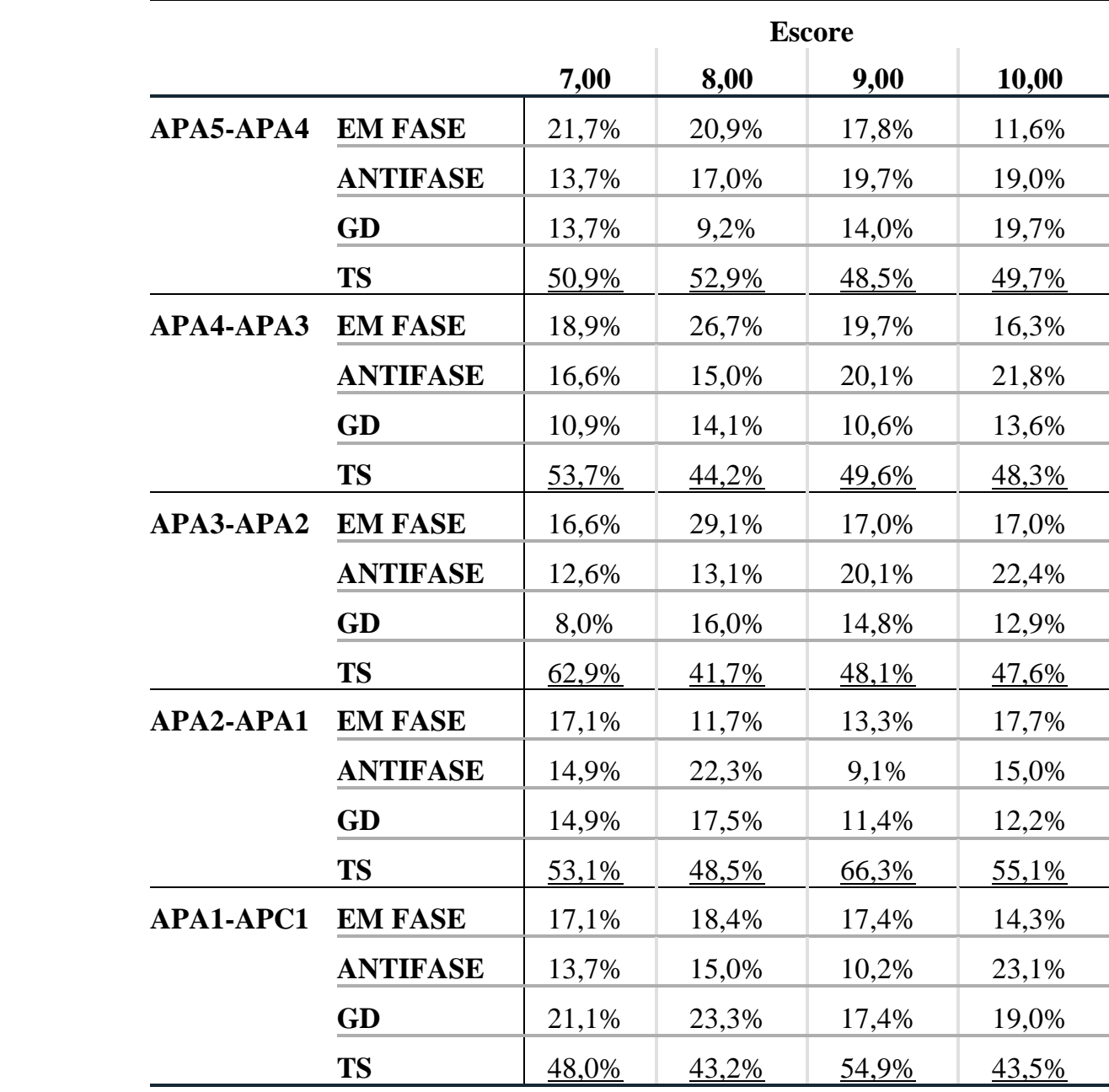

Fonte: Fernando Carvalheiro Reiser (2019) 
Tabela 54. Moda da fase de coordenação por escore

\begin{tabular}{cccccc}
\hline & APA5-APA4 & APA4-APA3 & APA3-APA2 & APA2-APA1 & APA1-APC1 \\
\hline 7 & TS & TS & TS & TS & TS \\
8 & TS & TS & TS & TS & TS \\
9 & TS & TS & TS & TS & TS \\
10 & TS & TS & TS & TS & TS \\
\hline
\end{tabular}

Fonte: Fernando Carvalheiro Reiser (2019)

4.4.4 Índices mistos:

O índice Serrátil Anterior - bilateral (SAD-SAnD) para análise do vetor codificado, nas respectivas frequências e modas dos tipos de fases de coordenação, de todas as flechas disparadas, do par de músculos $\mathrm{SAD}$ - antagonista, que traciona a corda e o agonista, que segura o arco - SAnD estão na figura 22. A moda de coordenação na transição do APA5-APA4, APA4-APA3, APA3-APA2, e APA2-APA1 foi de Fase do Serrátil Anterior dominante; na transição dos ajustes APA1-APC1 a Fase foi de Serrátil Anterior não-dominante. A totalidade dos casos foi dividida entre Fase do Serrátil Anterior dominante e de Fase do Serrátil Anterior - não dominante (32\% dos casos totais cada - APA5-APC1). Na tabela 55 está a análise por desempenho, com a moda de fase de coordenação destacada por APA-APC, e na tabela 56 estão as modas de transição de cada ajuste de maneira resumida.

Figura 22. Análise de Vetor Codificado das frequências, por percentuais, do índice SAD-SAnD

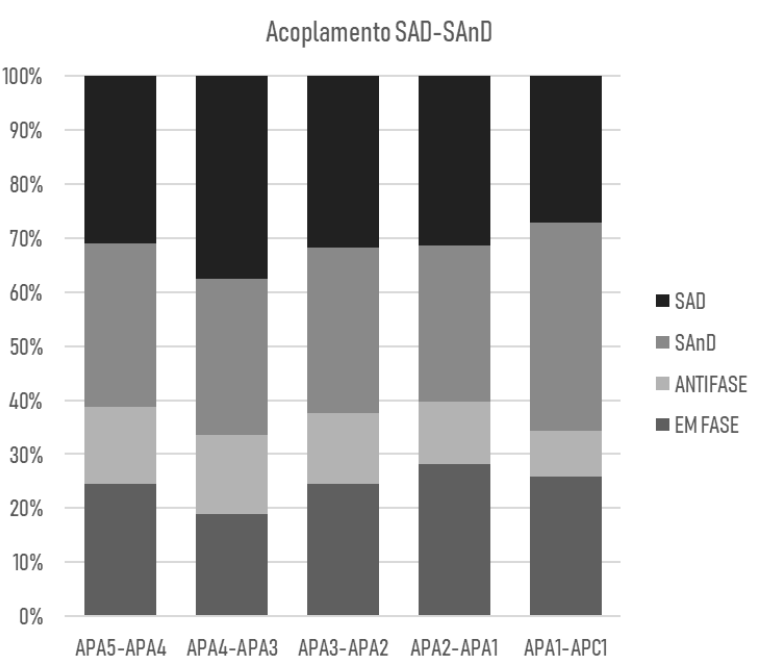

Fonte: Fernando Carvalheiro Reiser (2019) 
Tabela 55. Análise de Vetor Codificado - frequências, em percentis, por escore (a moda está sublinhada).

\begin{tabular}{|c|c|c|c|c|c|}
\hline & & \multicolumn{4}{|c|}{ Escore } \\
\hline & & 7,00 & $\mathbf{8 , 0 0}$ & 9,00 & 10,00 \\
\hline \multirow[t]{4}{*}{ APA5-APA4 } & EM FASE & $25,7 \%$ & $28,2 \%$ & $20,8 \%$ & $24,5 \%$ \\
\hline & ANTIFASE & $13,7 \%$ & $15,5 \%$ & $17,0 \%$ & $8,2 \%$ \\
\hline & SAnD & $26,9 \%$ & $22,8 \%$ & $34,8 \%$ & $36,1 \%$ \\
\hline & SAD & $33,7 \%$ & $33,5 \%$ & $27,3 \%$ & $31,3 \%$ \\
\hline \multirow[t]{4}{*}{ APA4-APA3 } & EM FASE & $17,7 \%$ & $22,3 \%$ & $15,9 \%$ & $21,1 \%$ \\
\hline & ANTIFASE & $12,6 \%$ & $18,0 \%$ & $17,4 \%$ & $6,8 \%$ \\
\hline & SAnD & $29,1 \%$ & $22,3 \%$ & $32,2 \%$ & $32,7 \%$ \\
\hline & SAD & $40,6 \%$ & $37,4 \%$ & $\underline{34,5 \%}$ & $39,5 \%$ \\
\hline \multirow[t]{4}{*}{ APA3-APA2 } & EM FASE & $24,6 \%$ & $24,8 \%$ & $27,3 \%$ & $19,0 \%$ \\
\hline & ANTIFASE & $18,3 \%$ & $12,6 \%$ & $10,2 \%$ & $12,9 \%$ \\
\hline & SAnD & $\underline{31,4 \%}$ & $\underline{32,5 \%}$ & $27,3 \%$ & $32,7 \%$ \\
\hline & SAD & $25,7 \%$ & $30,1 \%$ & $\underline{35,2 \%}$ & $\underline{35,4 \%}$ \\
\hline \multirow[t]{4}{*}{ APA2-APA1 } & EM FASE & $32,6 \%$ & $31,6 \%$ & $24,2 \%$ & $25,2 \%$ \\
\hline & ANTIFASE & $10,9 \%$ & $12,6 \%$ & $10,2 \%$ & $12,9 \%$ \\
\hline & SAnD & $\underline{33,7 \%}$ & $20,9 \%$ & $31,8 \%$ & $29,3 \%$ \\
\hline & SAD & $22,9 \%$ & $\underline{35,0 \%}$ & $\underline{33,7 \%}$ & $\underline{32,7 \%}$ \\
\hline \multirow[t]{4}{*}{ APA1-APC1 } & EM FASE & $29,7 \%$ & $27,2 \%$ & $22,7 \%$ & $25,2 \%$ \\
\hline & ANTIFASE & $9,7 \%$ & $6,8 \%$ & $8,3 \%$ & $9,5 \%$ \\
\hline & SAnD & $\underline{42,3 \%}$ & $\underline{42,7 \%}$ & $\underline{36,7 \%}$ & $31,3 \%$ \\
\hline & SAD & $18,3 \%$ & $23,3 \%$ & $32,2 \%$ & $\underline{34,0 \%}$ \\
\hline
\end{tabular}

Fonte: Fernando Carvalheiro Reiser (2019)

Tabela 56. Moda da fase de coordenação por escore

\begin{tabular}{cccccc}
\hline & APA5-APA4 & APA4-APA3 & APA3-APA2 & APA2-APA1 & APA1-APC1 \\
\hline 7 & SAD & SAD & SAnD & SAnD & SAnD \\
8 & SAD & SAD & SAnD & SAD & SAnD \\
9 & SAnD & SAD & SAD & SAD & SAnD \\
10 & SAnD & SAD & SAD & SAD & SAD \\
\hline
\end{tabular}

Fonte: Fernando Carvalheiro Reiser (2019)

O índice dos músculos que seguram o arco - da articulação da escápula e da articulação glenoumeral para análise do vetor codificado, nas respectivas frequências e modas dos tipos de fases de coordenação, de todas as flechas disparadas, do par de músculos SAnD - agonista da escápula e o agonista da glenoumeral DPnD - que seguram o arco estão na figura 23. A moda de coordenação na transição do APA5APA4 e APA4-APA3 foi de Fase do Deltóide Posterior - não-dominante; a transição dos ajustes APA3-APA2, APA2-APA1 e APA1-APC1 foi de Fase Serrátil Anterior 
não-dominante. A totalidade dos casos foi de Fase do Deltóide Posterior - não dominante (36\% dos casos totais- APA5-APC1). Na tabela 57 está a análise por desempenho, com a moda de fase de coordenação destacada por APA-APC, e na tabela 58 estão as modas de transição de cada ajuste de maneira resumida.

Figura 23. Análise de Vetor Codificado das frequências, por percentuais, do índice DPnD-SAnD

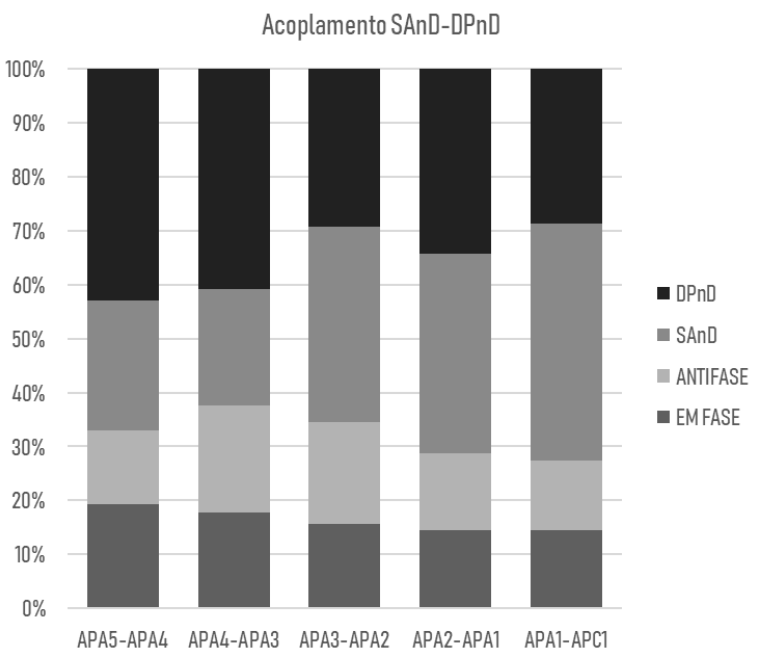

Fonte: Fernando Carvalheiro Reiser (2019)

Tabela 57. Análise de Vetor Codificado - frequências, em percentis, por escore (a moda está sublinhada).

\begin{tabular}{|c|c|c|c|c|c|}
\hline & & \multicolumn{4}{|c|}{ Escore } \\
\hline & & $\mathbf{7 , 0 0}$ & 8,00 & 9,00 & 10,00 \\
\hline \multirow[t]{4}{*}{ APA5-APA4 } & EM FASE & $20,0 \%$ & $13,6 \%$ & $23,5 \%$ & $19,0 \%$ \\
\hline & ANTIFASE & $18,9 \%$ & $13,6 \%$ & $12,1 \%$ & $10,2 \%$ \\
\hline & SAnD & $22,9 \%$ & $26,7 \%$ & $24,2 \%$ & $21,8 \%$ \\
\hline & DPnD & $38,3 \%$ & $\underline{46,1 \%}$ & $\underline{40,2 \%}$ & $49,0 \%$ \\
\hline \multirow[t]{4}{*}{ APA4-APA3 } & EM FASE & $24,6 \%$ & $14,1 \%$ & $18,6 \%$ & $13,6 \%$ \\
\hline & ANTIFASE & $16,6 \%$ & $18,9 \%$ & $23,9 \%$ & $17,7 \%$ \\
\hline & SAnD & $21,7 \%$ & $18,0 \%$ & $23,9 \%$ & $21,8 \%$ \\
\hline & DPnD & $\underline{37,1 \%}$ & $\underline{49,0 \%}$ & $\underline{33,7 \%}$ & $\underline{46,9 \%}$ \\
\hline \multirow[t]{4}{*}{ APA3-APA2 } & EM FASE & $19,4 \%$ & $16,0 \%$ & $13,3 \%$ & $15,0 \%$ \\
\hline & ANTIFASE & $16,6 \%$ & $19,9 \%$ & $20,5 \%$ & $17,0 \%$ \\
\hline & SAnD & $\underline{36,0 \%}$ & $30,1 \%$ & $\underline{40,9 \%}$ & $\underline{36,7 \%}$ \\
\hline & DPnD & $28,0 \%$ & $34,0 \%$ & $25,4 \%$ & $31,3 \%$ \\
\hline \multirow[t]{4}{*}{ APA2-APA1 } & EM FASE & $14,3 \%$ & $16,0 \%$ & $14,0 \%$ & $13,6 \%$ \\
\hline & ANTIFASE & $13,1 \%$ & $11,7 \%$ & $14,8 \%$ & $18,4 \%$ \\
\hline & SAnD & $\underline{44,6 \%}$ & $29,6 \%$ & $\underline{40,5 \%}$ & $31,3 \%$ \\
\hline & DPnD & $28,0 \%$ & $\underline{42,7 \%}$ & $30,7 \%$ & $\underline{36,7 \%}$ \\
\hline \multirow[t]{4}{*}{ APA1-APC1 } & EM FASE & $17,7 \%$ & $14,1 \%$ & $12,9 \%$ & $14,3 \%$ \\
\hline & ANTIFASE & $14,3 \%$ & $16,5 \%$ & $11,0 \%$ & $9,5 \%$ \\
\hline & SAnD & $\underline{42,9 \%}$ & $\underline{35,0 \%}$ & $\underline{49,6 \%}$ & $\underline{47,6 \%}$ \\
\hline & DPnD & $25,1 \%$ & $34,5 \%$ & $26,5 \%$ & $28,6 \%$ \\
\hline
\end{tabular}

Fonte: Fernando Carvalheiro Reiser (2019) 
Tabela 58. Moda da fase de coordenação por escore

\begin{tabular}{cccccc}
\hline & APA5-APA4 & APA4-APA3 & APA3-APA2 & APA2-APA1 & APA1-APC1 \\
\hline 7 & DPnD & DPnD & SAnD & SAnD & SAnD \\
8 & DPnD & DPnD & DPnD & DPnD & SAnD \\
9 & DPnD & DPnD & SAnD & SAnD & SAnD \\
10 & DPnD & DPnD & SAnD & DPnD & SAnD \\
\hline
\end{tabular}

Fonte: Fernando Carvalheiro Reiser (2019)

O índice dos músculos analisados extensores da articulação glenoumeral na análise do vetor codificado, com frequências e modas dos tipos de fases de coordenação, de todas as flechas disparadas, do par de músculos GD - DPnD, estão na figura 24. A moda de coordenação na transição do APA5 até o APC1 foi de Fase do Deltóide Posterior - não-dominante. A totalidade dos casos de Fase do Deltóide Posterior - não dominante (47\% dos casos totais- APA5-APC1). Na tabela 59 está a análise por desempenho, com a moda de fase de coordenação destacada por APA-APC, e na tabela 60 estão as modas de transição de cada ajuste de maneira resumida.

Figura 24. Análise de Vetor Codificado das frequências, por percentuais, do índice GD-DPnD

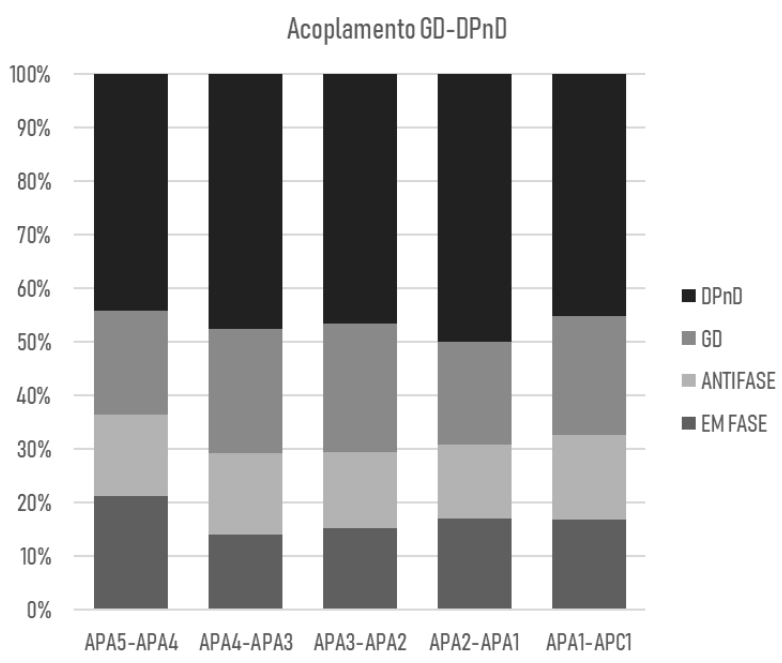

Fonte: Fernando Carvalheiro Reiser (2019) 
Tabela 59. Análise de Vetor Codificado - frequências, em percentis, por escore (a moda está sublinhada).

\begin{tabular}{|c|c|c|c|c|c|}
\hline & & \multicolumn{4}{|c|}{ Escore } \\
\hline & & 7,00 & 8,00 & 9,00 & 10,00 \\
\hline \multirow[t]{4}{*}{ APA5-APA4 } & EM FASE & $22,3 \%$ & $22,3 \%$ & $19,7 \%$ & $21,8 \%$ \\
\hline & ANTIFASE & $16,0 \%$ & $13,6 \%$ & $18,2 \%$ & $10,9 \%$ \\
\hline & GD & $20,0 \%$ & $21,4 \%$ & $19,3 \%$ & $15,6 \%$ \\
\hline & DPnD & $\underline{41,7 \%}$ & $\underline{42,7 \%}$ & $\underline{42,8 \%}$ & $\underline{51,7 \%}$ \\
\hline \multirow[t]{4}{*}{ APA4-APA3 } & EM FASE & $14,9 \%$ & $15,0 \%$ & $12,9 \%$ & $13,6 \%$ \\
\hline & ANTIFASE & $15,4 \%$ & $16,0 \%$ & $14,4 \%$ & $15,0 \%$ \\
\hline & GD & $18,9 \%$ & $23,3 \%$ & $29,2 \%$ & $18,4 \%$ \\
\hline & DPnD & $\underline{50,9 \%}$ & $\underline{45,6 \%}$ & $\underline{43,6 \%}$ & $\underline{53,1 \%}$ \\
\hline \multirow[t]{4}{*}{ APA3-APA2 } & EM FASE & $18,3 \%$ & $11,7 \%$ & $14,0 \%$ & $19,0 \%$ \\
\hline & ANTIFASE & $12,0 \%$ & $15,5 \%$ & $17,8 \%$ & $8,8 \%$ \\
\hline & GD & $22,3 \%$ & $27,2 \%$ & $23,1 \%$ & $22,4 \%$ \\
\hline & DPnD & $\underline{47,4 \%}$ & $\underline{45,6 \%}$ & $\underline{45,1 \%}$ & $\underline{49,7 \%}$ \\
\hline \multirow[t]{4}{*}{ APA2-APA1 } & EM FASE & $16,0 \%$ & $18,0 \%$ & $20,1 \%$ & $11,6 \%$ \\
\hline & ANTIFASE & $12,0 \%$ & $12,1 \%$ & $14,4 \%$ & $17,7 \%$ \\
\hline & GD & $22,3 \%$ & $16,5 \%$ & $20,5 \%$ & $16,3 \%$ \\
\hline & DPnD & $\underline{49,7 \%}$ & $\underline{53,4 \%}$ & $\underline{45,1 \%}$ & $\underline{54,4 \%}$ \\
\hline \multirow[t]{4}{*}{ APA1-APC1 } & EM FASE & $21,1 \%$ & $13,6 \%$ & $17,8 \%$ & $15,0 \%$ \\
\hline & ANTIFASE & $13,1 \%$ & $14,6 \%$ & $14,4 \%$ & $22,4 \%$ \\
\hline & GD & $18,9 \%$ & $23,3 \%$ & $25,0 \%$ & $19,7 \%$ \\
\hline & DPnD & $46,9 \%$ & $48,5 \%$ & $42,8 \%$ & $\underline{42,9 \%}$ \\
\hline
\end{tabular}

Fonte: Fernando Carvalheiro Reiser (2019)

Tabela 60. Moda da fase de coordenação por escore

\begin{tabular}{cccccc}
\hline & APA5-APA4 & APA4-APA3 & APA3-APA2 & APA2-APA1 & APA1-APC1 \\
\hline 7 & DPnD & DPnD & DPnD & DPnD & DPnD \\
8 & DPnD & DPnD & DPnD & DPnD & DPnD \\
9 & DPnD & DPnD & DPnD & DPnD & DPnD \\
10 & DPnD & DPnD & DPnD & DPnD & DPnD \\
\hline
\end{tabular}

Fonte: Fernando Carvalheiro Reiser (2019)

\subsection{Aspectos globais de análise}

A tabela 61 sintetiza os resultados encontrados pelo presente estudo, que dá-nos subsídios para confrontar as hipóteses levantadas; de modo geral é possível deduzir que para a maioria dos índices analisados alguma diferença foi encontrada, com alguns casos ocorrendo na transição de blocos, e com a maioria das modulações ocorrendo no desempenho; também num caso de inibição reciproca foi também encontrada diferenças para a análise individual de flechas. A mesma tendência, no caso de modificação de 
fases de coordenação, pode ser visualizada na análise de Vetor Codificado, com transições de coordenação na análise temporal, como nas de desempenho (escores).

Tabela 61. Resumo dos resultados encontrados em todos os índices analisados

\begin{tabular}{|c|c|c|c|c|c|c|c|c|c|c|c|c|c|c|}
\hline \multirow[t]{2}{*}{ Índice } & \multicolumn{6}{|c|}{ Co-Ativação } & \multicolumn{6}{|c|}{ Inibição Recíproca } & \multicolumn{2}{|c|}{ AVC } \\
\hline & APA5 & APA4 & APA3 & APA2 & APA1 & APC1 & APA5 & APA4 & APA3 & APA2 & APA1 & APC1 & Total & Escore \\
\hline FSD-ED & & & & *\# & $*$ & *\# & & & & *\# & $*$ & $\#$ & $\neq$ & $\neq$ \\
\hline BBL-TBL & & & *\# & $\#$ & & \# & & & *\# & $*$ & $*$ & $*$ & $=$ & $\neq$ \\
\hline GD-PMC & & & & & $\#$ & & & & & & & & $\neq$ & $\neq$ \\
\hline TS-SAD & & & & $\#$ & *\# & & $*$ & & + & & $*$ & & $=$ & $\neq$ \\
\hline RA-ML & & & & & & & & & & & & & $=$ & $=$ \\
\hline FSD-BBL & \# & \# & *\# & & & *\# & & & *\# & $*$ & & \# & $\neq$ & $\neq$ \\
\hline FSD-TBL & & & *\# & $\#$ & & \# & & & *\# & $\#$ & \# & \# & $\neq$ & $\neq$ \\
\hline ED-BBL & & \# & \# & \# & \# & & & \# & *\# & \# & & & $\neq$ & $\neq$ \\
\hline ED-TBL & & \# & $* \#$ & \# & $\#$ & \# & & & *\# & \# & & $\#$ & $\neq$ & $\neq$ \\
\hline PMC-SAD & & & & \# & $\#$ & \# & & & & & & & $\neq$ & $\neq$ \\
\hline GD-TS & & & & & & & & & & & & & $=$ & $=$ \\
\hline SAD-SAnD & *\# & $*$ & $*$ & *\# & \# & \# & $* \#$ & $*$ & $*$ & *\# & \# & \# & $\neq$ & $\neq$ \\
\hline SAnD-DPnD & $* \#$ & \# & & & & & *\# & \# & & & & & $\neq$ & $\neq$ \\
\hline GD-DPnD & \# & & $\#$ & & & & $\#$ & & \# & & & & $=$ & $=$ \\
\hline
\end{tabular}

Fonte: Fernando Carvalheiro Reiser (2019)

Legendas: Diferenças nos blocos (*); Flechas (+); Escores (\#); A.V.C. (Análise de Vetor Codificado) mesma moda coordenação durante todas as transições $(=)$; mais de uma moda de coordenação nas transições $(\neq)$. 


\section{DISCUSSÃO}

Retomando o objetivo principal do presente trabalho, de avaliar o desempenho de arqueiros da seleção brasileira por meio de diferentes abordagens de controle motor na atividade eletromiográfica, evidencia-se que ambas as possibilidades, partindo de origens diferentes, levam-nos a algumas considerações similares no que tange os parâmetros temporais e desempenho nos arqueiros de elite.

A síntese na tabela 61, ajuda-nos a confrontar as hipóteses do presente trabalho, e retomando a elas verificamos, para a HPT, se os mecanismos de co-ativação nos ajustes foram os mesmos, ou diferentes, para as análises dos blocos, flechas e de desempenho. No mesmo caso, verificamos se os mecanismos de inibição recíproca nos ajustes serão os mesmos, ou diferentes, para as análises dos blocos, flechas e de desempenho, e para os Sistemas Dinâmicos, se os arqueiros manteram um tipo de fase de coordenação, ou modificaram-no nos ajustes na análise dos blocos, flechas e de desempenho.

Para os índices de co-ativação, a hipótese nula deve ser aceita no que tange a modificações de modulação na transição de blocos, flechas e na análise de desempenho em dois pares RA-ML e GD-TS, na qual não foram encontrados efeitos de modulação de comando $\mathrm{C}$ nestes aspectos. A hipótese de nulidade é aceita com possíveis influências dois fatores; ou de fato a modulação é mais constante de co-ativação não exercendo influência na transição bloco-a-bloco, flecha e desempenho obtido, ou a variabilidade de modulação destes resultados, na comparação conjunta de arqueiros, foi demasiada para detectar tais alterações. Individualmente e para cada flecha, todos os índices de co-ativação não sofreram efeito, aceitando-se a hipótese de nulidade, evidenciando que ou a variabilidade na ordem de disparo da flecha dilui o efeito desta variável ou que existe alguma consistência temporal na maioria dos casos analisados. $\mathrm{Na}$ mesma tendência observacional destes fatores tiveram quatro índices de inibição reciproca, RA-ML, GD-PMC, PMC-SAD e GD-TS, onde deve-se aceitar a hipótese de nulidade, pelos resultados não significativos encontrados na análise de blocos, flechas e escores obtidos, para a análise da ordem de disparo da flecha, em treze índices deve-se aceitar a hipótese de nulidade (FSD-ED, BBL-TBL, GD-PMC, RA-ML, FSD-BBL, FSD-TBL, ED-BBL, ED-TBL, PMC-SAD, GD-TS, SAD-SAnD, SAnD-DPnD e GD$\mathrm{DPnD}$ ), não tendo efeito de modulação no comando R nestes pares de músculos. 
Nos demais casos deve-se aceitar a hipótese alternativa, pois ao menos uma modulação diferente foi observada ou na passagem bloco-a-bloco ou desempenho nalgum ajuste postural antecipatório ou compensatório. Deve-se aceitar a hipótese alternativa nos índices de co-ativação para transição de blocos nos seguintes oito pares, FSD-ED, BBL-TBL, TS-SAD, FSD-BBL, FSD-TBL, ED-TBL, SAD-SAnD e SAnD$\mathrm{DPnD}$, evidenciando que de fato existe blocos que os arqueiros realizam maior coativação como estratégia de modulação. Nos escores se deve aceitar a hipótese alternativa em 12 índices, FSD-ED, BBL-TBL, GD-PMC, FSD-BBL, FSD-TBL, EDBBL, ED-TBL, PMC-SAD, TS-SAD, SAD-SAnD, SAnD-DPnD e GD-DPnD, evidenciando que os arqueiros co-ativam em maior ou menor proporção estes pares de músculos para obtenção de melhores pontuações nos ajustes posturais analisados.

Para inibição recíproca deve-se aceitar a hipótese alternativa para transição de blocos nos seguintes nove pares, FSD-ED, BBL-TBL, TS-SAD, FSD-BBL, FSD-TBL, ED-BBL, ED-TBL, SAD-SAnD e SAnD-DPnD, evidenciando que existem blocos que os arqueiros realizam a inibição recíproca como estratégia de modulação. Um índice sofreu efeito das flechas na inibição reciproca (TS-SAD), evidenciando que os arqueiros utilizam diferentes estratégias de coordenação em músculos periescapulares na ordem de tiro das flechas dentro do bloco, aceitando-se a hipótese alternativa. Nos escores, evidencia-se que se deve aceitar a hipótese alternativa em nove índices, FSD-ED, BBLTBL, FSD-BBL, FSD-TBL, ED-BBL, ED-TBL, SAD-SAnD, SAnD-DPnD e GD$\mathrm{DPnD}$, evidenciando que os arqueiros realizam a inibição recíproca em maior ou menor proporção estes pares de músculos para melhorar a pontuação. É possível que parte do grupo analisado de arqueiros use maior proporção estratégia de co-ativação e outra de inibição recíproca, ou até mesmo o mesmo arqueiro utilizando ambas estratégias em diferentes momentos conforme a tarefa.

$\mathrm{Na}$ literatura, há um imbróglio na detecção do maior e menor desempenho desses atletas. Suwarganda et al. (2012) observaram em três arqueiros de elite que a atividade muscular do trapézio do lado direito e do tríceps braquial do lado esquerdo determinavam a pontuação de um atleta, outro atleta mostrou a atividade mais intensa de deltóide do lado esquerdo quando a pontuação elevava, e outro arqueiro, o mais experiente do grupo, alcançou o escore mais alto quando a atividade muscular do tríceps braquial do lado direito era mais intensa. Nishizono et al. (1987) evidenciaram a estratégia inibição recíproca da atividade do músculo deltóide acromial após o lançamento da flecha em dois arqueiros de elite. Na análise individual de Tinazci (2011), nota-se as diferentes relações entre desempenho e eletromiografia em arqueiros, 
um deles atingia maior desempenho quando diminuía a atividade muscular do FSD, TS e ED imediatamente antes da queda do clicker, noutro isto ocorria com maior atividade dos ED, e no terceiro atleta, isto ocorria quando diminuía a atividade dos FSD somente. Desta forma, existem estratégias de inibição recíproca e co-ativação heterogêneas em arqueiros de elite, com alguns aspectos similares entre eles.

$\mathrm{Na}$ análise de Vetor Codificado a moda de cinco índices foi a coordenação de fase: BBL-TBL; TS-SAD; RA-ML; GD-TS e GD-DPnD. Para análise global das flechas, na análise de desempenho, isto ocorreu para nos índices RA-ML; GD-TS e GDDPnD. Nestes casos, tanto no total de flechas disparadas como na análise de pontuação, deve-se aceitar a hipótese de nulidade pois a moda de coordenação não sofreu efeito destes aspectos temporais e de desempenho. Isto indica nestes pares de músculos, os arqueiros de elite adotam com maior frequência um tipo de coordenação para obter escores mais ou menos elevados como na transição de tiro-a-tiro disparado.

Em nove índices (FSD-ED, GD-PMC, FSD-BBL, FSD-TBL, ED-BBL, EDTBL, PMC-SAD, SAD-SAnD e SAnD-DPnD), deve-se aceitar a hipótese alternativa, pois houve efeito na transição de ajustes posturais, com ora fase de determinado músculo ora de outro. 11 índices (FSD-ED, BBL-TBL, GD-PMC, FSD-BBL, FSDTBL, ED-BBL, ED-TBL, PMC-SAD, SAD-SAnD e SAnD-DPnD) tiveram diferentes tipos de coordenação na obtenção dos escores no desempenho, onde também deve-se aceitar a hipótese alternativa, o que evidencia que arqueiros de elite também acionam em maior ou menor intensidade alguns músculos quando obtém resultados melhores.

Nota-se a heterogeneidade de estratégias dos arqueiros de alto-rendimento, o que aumenta a complexidade da análise temporal e de desempenho. Pouquíssimas são as relações lineares. Apesar de algumas modas de coordenação estarem relacionadas com desempenhos inferiores, é possível atingir pontuações elevadas com essas coordenações com, evidentemente, menor frequência. A análise de Vetor Codificado havia sido implementada somente em dados cinemáticos, identificando os tipos de coordenação no ataque do pé ao solo durante a marcha (CHANG; VAN EMMERIK; HAMILL, 2008), o sentido da coordenação proximal-distal e distal-proximal em homens e mulheres (RODRIGUES et al., 2015), e a curva em U, relacionada ao desempenho de atletas de salto em distância com diferentes níveis de aptidão (WILSON et al., 2008). Este trabalho é pioneiro pois usou esta ferramenta na eletromiografia em atletas de tiro com arco, evidenciando em pares de músculos, as estratégias utilizadas por arqueiros na obtenção do desempenho; a complementação desta análise com as modulações de comando C e R serão discutidos individualmente a seguir. 
$\mathrm{Na}$ análise dos pares agonistas-antagonistas nos músculos do punho FSD-ED, não houve efeito na análise bloco-a-bloco do período APA5, APA4 e APA3, já no período de APA2, APA1 e APC1, além do aumento de co-ativação no índice, as diferenças ocorreram envolvendo o bloco 3 em comparações pontuais com demais blocos. Estes resultados evidenciam que os atletas co-ativam estes músculos modulando cada sequência de blocos, e estas modulações só são evidentes após o período pré-APA (-500ms a -250ms antes da queda do clicker) principalmente nos últimos momentos antes da efetivação do disparo. Não houve diferença para a variável flecha em todos os ajustes posturais analisados, isto se deve a fatores mencionados como a variabilidade entre arqueiros ou a consistência de co-ativação que não sofre efeito desta variável. Esses dados convergem com a perspectiva de que as existem diferentes estratégias do FSD e ED nesses ajustes imediatamente pré e pós clicker, contudo elas também ocorrem temporalmente bloco-a-bloco, dado ainda não evidenciado pela literatura (ERTAN et al., 2003, 2011; SOLYU et al., 2006; MARTIN; SILER; HOFFMAN, 1990).

Para a atividade muscular dos flexores e extensores do punho e dos dedos, no tiro com arco, sugere que após a liberação da corda o relaxamento dos músculos flexores dos dedos seria o suficiente para a extensão dos dedos, cerca de 100-200ms pós-queda do clicker - Only Flexor's Strategy - estratégia prioritariamente de inibiçãorecíproca (MARTIN; SILER; HOFFMAN, 1990; ERTAN et al., 2011). Contudo, existem arqueiros de elite que não utilizam a estratégia de relaxamento, produzindo um pico de atividade dos ED logo após a queda do clicker - estratégia de co-ativação (Active Extensor's Strategy) - desta forma, alguns arqueiros de elite utilizam ambas as estratégias, principalmente pelo fato que existem diferenças de modulação ao longo da sequência de 72 tiros na análise bloco-a-bloco (HENNESSY \& PARKER 1990; MARTIN; SILER; HOFFMAN, 1990; ERTAN et al. 2003; SOYLU et al. 2006; SHINOHARA, URABE, 2017).

No desempenho, as diferenças ocorreram no APA2, com maior co-ativação no escore 9 em comparação ao 7 e no primeiro ajuste compensatório (APC1), maior no escore 10 em comparação com 7 e 8; e 9 maior que 7, ou seja quando analisamos o desempenho, existem diferentes co-ativações no ajuste -150 a $-50 \mathrm{~ms}$ antes da queda do clicker, estabilidade de modulação no APA1, e 50-150ms após a queda do cliker coativações diferentes. Esses dados não corroboram com Tinazci (2011) que correlacionou a atividade muscular de FSD e ED ao desempenho. Os dados de quatro arqueiros evidenciavam que, conforme a pontuação aumentava a atividade dos músculos FSD e 
ED diminuía. É possível que isso se deva a janela analisada do movimento. Analisamos o primeiro ajuste postural compensatório (APC) até o período de $150 \mathrm{~ms}$ após queda do clicker, onde a atividade do FSD e do ED pode estar elevada (ERTAN et al., 2003, 2011; MARTIN; SILER; HOFFMAN, 1990), a tendência é a diminuição até 500ms, donde Tinazci (2011) retirou a média da janela total (0-500ms após a queda do clicker).

No índice FSD-ED, não foram evidenciadas diferenças nos blocos do período APA5, APA4 e APA3, e no primeiro ajuste compensatório (APC1), já no período de APA2, APA1, as diferenças ocorreram envolvendo o bloco 3, em comparações pontuais com demais blocos (tabela 6). Ou seja, é possível um grupo de atletas utilize diferentes estratégias de inibição após o período pré-APA, mas que não se sustenta (bloco-a-bloco) no ajuste compensatório, o que é evidenciado na literatura (Only Flexor's Strategy) em função da variabilidade da atividade antagonista nesta fase, com arqueiros desempenhando atividades de co-ativação e outros de inibição recíproca, ou mesmo ambos (MARTIN; SILER; HOFFMAN, 1990). Não houve diferença para a variável flecha em todos os ajustes posturais analisados, corroborando com as perspectivas de variabilidade ou consistência apresentadas.

$\mathrm{Na}$ análise do desempenho, as diferenças ocorreram no APA2, no escore 9 comparado ao 7 e no primeiro ajuste compensatório (APC1) no 10 e 9 comparado ao 7. As modulações que ocorreram na análise do desempenho evidenciam que, apesar das modificações temporais (bloco-a-bloco) serem pontuais, as de desempenho são modificadas na comparação da atividade nos escores, não imediatamente antes do clicker (APA1), mas no primeiro ajuste compensatório (APC1) entre 10 e 9 versus 7 para o par analisado. Quando o arqueiro dispara a flecha no intervalo de $150-200 \mathrm{~ms}$ após a queda do clicker são observáveis diferenças em atletas de diferentes níveis de rendimento nos músculos FSD e ED (ERTAN et al., 2003; SOYLU et al. 2006; MARTIN; SILLER; HOFFMAN, 1990); arqueiros de elite podem atingir mais frequentemente um pico de atividade muscular, principalmente em ED, que arqueiros menos experientes. No presente estudo, apesar de todos os arqueiros serem considerados de alto rendimento, quando submetidos ao protocolo experimental também tiveram a atividade muscular insuficiente ou retardada quando considerada a análise de desempenho.

Para FSD-ED, na transição de APA5 até APC1, a o tipo de coordenação foi a Fase do ED. Somente na transição APA1-APC1, foram encontradas duas modas de coordenação (Fase do FSD seguida pela Fase ED). Os resultados evidenciam o uso das estratégias Only Flexor's Strategy, estratégia de Fase do FSD, e a Active Extensor's 
Strategy de Fase do ED (ERTAN et al., 2003, 2011; MARTIN; SILER; HOFFMAN, 1990), e predominantemente temos essa transição sendo utilizada na moda geral de tiros disparos (Fase de ED).

A mesma condição não foi observada na análise dos escores, com observações bastante heterogêneas. $O$ tipo de coordenação predominante na pontuação 7 e 9 foi fase ED; com aumento da atividade muscular do ED, e manutenção do FSD, não ocorreu a mesma tendência para o 8 com modificações para uma coordenação fase de FSD, e 10; que se inicia em fase (os músculos co-ativando), com transição APA4-APA2 - fase ED; e fase FSD do APA2-APC1. Estes dados ampliam a discussão acerca da estratégia que predomina o tiro é a coordenação em fase do ED, apesar de sê-la predominante nos escores 7 e 9, não são nos 8 e 10; já que estes resultados evidenciam que o FSD também é muitíssimo modulado e tem um papel importante no desempenho esportivo de arqueiros, elevando a atividade muscular com manutenção da atividade em ED, fator que não é claramente evidenciado (TINAZCI, 2011; HENNESSY \& PARKER 1990; MARTIN; SILER; HOFFMAN, 1990; ERTAN et al. 2003; SOYLU et al. 2006).

No índice do par de músculos do cotovelo BBL-TBL, a tendência é semelhante aos do punho, sem diferenças na análise de blocos na transição de ajustes APA5 e APA4. No instante pré-APA (500-250ms antes da perturbação), não existe diferença de modulação na transição de blocos, contudo no primeiro instante do APA (APA3) 250$150 \mathrm{~ms}$ antes da perturbação existe diferença com maior índice no bloco 4 quando comparado ao 11 e 12, depois a modulação de co-ativação estabiliza-se nos demais ajustes (APA2, APA1 e APC1). Neste índice, podemos destacar essa ação no primeiro ajuste (APA3), o papel do músculo Bíceps Braquial, que quando o úmero está abduzido com rotação interna, a cabeça longa se move na direção anterior e produz força posterior, o que impõe uma força compressiva a articulação aumentando a estabilidade anterior da articulação, ou seja é possível que este músculo no tiro com arco, age garantindo estabilização depois a atividade é mantida sem maiores oscilações, nos demais ajustes posturais antecipatórios até os compensatórios. Contudo, deve-se ressaltar que uma ativação mais pronunciada deste músculo pode ser um indicativo de instabilidade ou de lesão na articulação glenoumeral (FLESIG et al., 1995; HORSLEY, HERRINGTON, ROLF, 2010).

Nenhuma diferença foi evidenciada na análise das flechas, o que está em consonância com os aspectos já colocados na confrontação de hipóteses. A análise dos escores evidenciou diferenças somente a partir dos 250ms pré-clicker no APA3 e APA2, maior no escore 10 em comparação com 7, 8 e 9; e APC1: maior no 10 em 
comparação com 7. As modificações de desempenho neste índice podem estar mais relacionadas mais com o papel da cabeça longa do Tríceps, pois o Bíceps Braquial produz alguma estabilidade articular e tem a atividade muscular pouco modulável, em alguns casos ela pode ser menor conforme o nível de desempenho do atleta (NISHIZONO et al., 1987; SQUADRONE, RODANO, GALLOZZI, 1994; SHINOHARA, URABE, 2017). Suwarganda et al. (2012) evidenciaram, em arqueiros de elite, que o Tríceps Braquial, de ambos lados, determinava o desempenho do atleta conforme aumentava a sua atividade, corroborando com a análise deste índice no presente trabalho. A importância deste músculo, em particular da cabeça longa, é a ação concêntrica na abdução horizontal e extensão do ombro na puxada que traciona a corda do arco, em ação mais proximal do que distal (SOYLU; ERTAN; KORKUSUZ, 2006).

Os resultados do índice BBL-TBL de inibição recíproca não evidenciaram diferenças nos ajustes: APA5, APA4 e APA2, APA1 e APC1. Com diferenças somente no primeiro ajuste (APA3), maior no bloco 4 quando comparado aos blocos 1-3;6;8-12. $\mathrm{Na}$ inibição recíproca, o módulo deste índice favoreceu em maior proporção a atividade antagonista do Tríceps Braquial, evidenciando que este músculo tem mais peso na análise do par do cotovelo que o Bíceps Braquial (SOYLU, ERTAN, KORKUSUZ, 2006). Nenhum resultado significativo para a análise de flecha, em consonância com os aspectos mencionados.

$\mathrm{Na}$ análise de desempenho, modificações ocorreram a partir dos 250ms antes da perturbarção (queda do clicker), até os $150 \mathrm{~ms}$ após a perturbação. No APA3 e APA2, maior índice foi evidenciado no escore 10 em comparação com 7,8 e 9. No APA1 e APC 1: foi maior no 10 e 9 em comparação com 7. De modo geral, as diferenças ocorreram semelhantemente ao índice de co-ativação, evidenciando que estratégias de co-ativação e inibição recíproca neste par podem ser utilizadas arqueiros de alto rendimento, destaca-se a pronunciada atividade muscular do Tríceps Braquial cabeça longa em escores mais elevados, reforçando que este músculo é um dos componentes no tiro que podem influenciar o desempenho positivo ou negativo de atletas (SUWARGANDA et al. 2012). O Bíceps Braquial, apesar de ter um importante papel na estabilização da articulação glenoumeral, tem atividade muscular mais pronunciada em arqueiros menos experientes em comparação com os de elite, e é possível que a maior co-ativação deste músculo pode indicar maior instabilidade ou mesmo lesão, o que diminui o rendimento do atleta (NISHIZONO et al. 1987; SQUADRONE; RODANO; GALLOZZI, 1994; SHINOHARA, URABE, 2017; FLESIG et al., 1995; HORSLEY, HERRINGTON, ROLF, 2010). 
A análise de Vetor Codificado do par BBL-TBL indicou que na transição geral do total de flechas foi a Fase do TBL, reforçando os aspectos de consistência já apresentado anteriormente (SOYLU, ERTAN, KORKUSUZ, 2006).

$\mathrm{Na}$ análise dos escores, os mais elevados 10 e 9 tiveram moda de coordenação predominantemente de Fase do TBL; enquanto os outros dois tiveram mais alternância do padrão de coordenação; no 8 a transição APA5-APA3 foi de Fase do TBL, APA3APA1 - fase BBL, e APA1-APC1 fase TBL; no escore 7 a alternância foi de Em Fase (APA5-APA4) para Fase TBL (APA4-APA3), novamente Em Fase (APA2-APA1) e Fase do BBL na transição APA2-APC1. Os resultados do par de músculos do cotovelo evidenciam a tendência já apresentada nos índices de co-ativação e de inibição recíproca, como os dados da literatura, que corroboram que o músculo BBL pode ser mais responsivo por desempenho inferior quando é mais solicitado. Isto é visualizado em arqueiros de menor desempenho, em comparação com menos habilidosos (SHINOHARA, URABE, 2017; NISHIZONO et al. 1987), a fadiga é outro fator que pode, pela deteriorização mecânica, aumentar a atividade deste músculo, o que diminui o desempenho (SQUADRONE; RODANO; GALLOZZI, 1994). Shinohara \& Urabe, (2017) evidenciam que em arqueiros menos experientes a articulação do ombro tem maior ângulo de flexão de cotovelo e abdução do ombro, o que é um dos fatores que podem aumentar a ação agônica no cotovelo e sua estabilização glenoumeral pela sua origem escapular, é possível que a maior instabilidade da articulação pode levar a desempenhos inferiores. Contudo, o presente estudo não realizou a análise cinemática que corrobore para tais alegações. Por fim, a atividade do TBL está envolvida com desempenhos mais elevados, possivelmente pela a ação agonista biarticulada na articulação glenoumeral, corroborando com Suwarganda et al. (2012).

$\mathrm{Na}$ análise do índice GD-PMC, para co-ativação, não foram observadas diferenças na modulação de bloco a bloco, e flechar a flecha, em todos os instantes analisados (APA5, APA4, APA3, APA2, APA1 e APC1), indicando que temporalmente não existe diferença de modulação destes fatores. Contudo, nos escores a única observação dessas diferenças ocorreu na comparação do escore 10 e 8 maior em comparação ao 9 para o APA1, sem diferenças nos demais. Essa modulação pontual do índice nos escores evidencia que a ação antagônica deste par de músculos é pouco modulável, e equilibra-se no último instante compensatório analisado em todas as condições analisadas. O músculo PMC possui uma atividade constante durante o tiro com arco, e pouco modulável temporalmente na análise de blocos e flechas, exemplificando o fato que outros estudos não encontraram diferenças na ativação deste 
músculo em momentos pré e pós clicker (NISHIZONO et al. 1987; EDELMANNNUSSER \& GOLLHOFER, 1998; SIMSEK et al., 2014).

Foi observada a mesma tendência no par GD-PMC, para a inibição recíproca. Não foram encontrados efeitos dos blocos, flechas e escores para este índice, ou seja, a modulação não é diferente na comparação temporal e na análise de desempenho. Neste par, foi observado que ação antagonista do PMC teve maior atividade muscular que a agonista do GD. É possível que a ação concêntrica conjunta de redondo menor, latíssimo do dorso, tríceps braquial cabeça longa deltoide médio e posterior, conferem redundância a puxada da corda, dessa forma, o PMC pode agir numa ação antagônica e estabilizadora no acoplamento de forças que agem na articulação glenoumeral (KAPANDJI, 2007; HAMILL \& KNUTZEN, 2014; SOYLU; ERTAN; KORKUSUZ, 2006). Ackland et al., (2008) observaram que o músculo Peitoral Maior, na porção superior clavicular desenvolve o torque máximo até $120^{\circ}$ de abdução, tanto no plano coronal como no escapular. Shinohara et al. (2018) evidenciaram que o ângulo máximo da articulação glenoumeral utilizado por arqueiros de elite situa-se entre 105-95 graus. Logo, é plausível que a atividade redundante dos músculos que executam a puxada extensores da articulação glenoumeral - precisa ser estabilizada por potente co-ativação antagonista para estabilização do segmento.

A transição de coordenação dos ajustes inicialmente, do par GD-PMC, foi de Fase do GD (APA5-APA3) para Fase do PMC (APA3-APC1). A tendência é de no momento pré-APA (500-250 antes da queda do clicker) uma coordenação que favorece a atividade agonista que traciona a corda. No APA até o APC1, a tendência se inverte com maior ativação antagonista na articulação glenoumeral, deste par, que tende a estabilizar o disparo. $\mathrm{Na}$ análise dos escores, em todas as pontuações, tem-se inicialmente em Fase do GD no APA5-APA4; no escore 7 e 9 mantém-se esta coordenação, com modificação para Fase do PMC depois do pré-APA (APA3-APA2) até o APC. No escore 8, esta mesma modificação ocorre na transição APA4-APA3. No escore 10, temos a coordenação que também inicia-se em Fase do GD no pré-APA (APA5-APA4), modifica-se para Em Fase (APA4-APA3) e depois mantém em Fase do PMC em todas as demais transições, ou seja no momento pré-APA (APA5-APA4; APA4-AP3) existe maior variabilidade de coordenação na análise de desempenho, depois tem-se a consistência da atividade antagonista predominantemente, exemplificando a ação pouco modulável deste músculo (NISHIZONO et al. 1987; EDELMANN-NUSSER \& GOLLHOFER, 1998; SIMSEK et al., 2014). 
A estabilização da articulação glenoumeral depende da ação dos músculos periescapulares. O par TS-SAD foi analisado neste intuito. Os resultados não apontaram diferenças no Pré-APA e nos primeiros APA (do APA5 ao APA2), com modulação consistente; contudo, no APA1, diferenças foram encontradas, evidenciando maior modulação nos blocos 4 e 6 em comparação com o 9. Essas diferenças não foram encontradas no APC1. Temporalmente diferenças só foram encontradas neste último ajuste pré-queda do clicker. O interessante deste resultado é que é esperado o resultado inverso na literatura, principalmente no músculo Trapézio Superior; onde Squadrone, Rodano \& Gallozzi, (1994) observaram seu aumento em atletas iniciantes e de elite após um protocolo de fadiga. Não analisamos este fator fisiológico, contudo a atividade deste índice na co-ativação foi menor no bloco 9, que pertence ao quarto final do protocolo. Possivelmente arqueiros de alto rendimento modulem melhor este músculo, com estratégias que atenuam a deterioriação mecânica mantendo o desempenho. Nenhum efeito para flecha, o que confere consistência a modulação na ordem das flechas.

Nos escores, não foram encontradas diferenças nos ajustes primeiros do préAPA ao primeiro APA, exceto entre APA 2 e APA1; sendo maiores no escore 10 quando comparado ao 7 (APA2) e maiores no 9 em comparação a 10 e 8 (APA1). No APC1 isso não ocorreu. Nas ondulações do desempenho, os arqueiros de elite costumam acionar em menor intensidade o músculo Trapézio Superior em comparação a arqueiros iniciantes (SHINOHARA, URABE, 2017), isto também pode ser verificado no desempenho de arqueiros de elite, na comparação do APA2 (150-50ms antes da perturbação) onde a co-ativação teve maior intensidade na pontuação mais elevada (10) e menor na pior pontuação obtida (7). Mas, verificou-se que a co-ativação no APA1 dobra em intensidade no escore 10, e chega a ser até 5 vezes maior nos demais, ou seja apesar da grande variabilidade no desempenho de arqueiros de elite neste músculo (SUWARGANDA et al. 2012; SIMSEK et al. 2014), nota-se a modulação para obter escores melhores na transição dos ajustes.

Foi encontrado efeito na inibição recíproca, já no momento pré-APA - APA5; com diferenças entre os blocos 1 e 2, com menor modulação comparado ao 12; e no APA1, e menor no bloco 9 em comparação ao 4; sem diferenças nos demais APA (APA4, APA3, APA2 e APC1). O comando R nos músculos periescapulares tem maior modulação e maior variabilidade por serem estes músculos estabilizadores que dão suporte a todo o restante da cadeia de movimento em membros superiores. Nas flechas, foram encontradas diferenças no APA3 (menores flecha 3 em comparação a 5), bloco-abloco e flecha-a-flecha neste índice o arqueiro modula e procura a melhor estabilidade 
para o disparo. O Trapézio Superior é um agonista importante para o tiro e pode se tornar um empecilho se ativado em maior intensidade. Shinohara \& Urabe (2017) encontraram que a razão de atividade muscular entre Trapézio Superior/Trapézio Inferior em arqueiros de elite e iniciantes é diferente (1,23 e 2,33 respectivamente).

Não houve diferença na análise de desempenho para inibição recíproca no TSSAD. A estratégia que predomina no desempenho de arqueiros de elite é co-ativação. $\mathrm{Na}$ análise de Vetor Codificado, a moda predominante em todos os ajustes (APA5APC1) foi de Fase do TS. A coordenação predominante nos escores menores (7 e 8) foi de Fase do TPS, no escore 9 a tendência permaneceu, com coordenação Fase do SAD pontual na transição APA3-APA2 (pós o pré-APA). No escore 10, a transição APA5APA3 foi Fase TPS e inverteu para Fase SAD nos ajustes finais APA3-APC1. A análise do escore mais elevado sintetiza as observações feitas nos índices de co-ativação. O $\mathrm{SAD}$, apesar de antagonista no movimento do disparo, também estabiliza a escápula a segurando contra a caixa torácica, principalmente em movimentos acima de $90^{\circ}$ da abdução com rotação externa da articulação glenoumeral (EKSTOM, et al. 2003; 2005). O aumento da atividade deste estabilizador periescapular com a manutenção da atividade do TPS converge com a hipótese de que essa coordenação leva o atleta a atingir maior desempenho no tiro com arco, tendo em vista que a sua atividade equilibrada ocorre em atletas de maior desempenho (SHINOHARA \& URABE, 2017; SQUADRONE; RODANO; GALLOZZI, 1994; SUWARGANDA et al. 2012).

Clarys et al. (1990) corroboram com essa perspectiva. Avaliando arqueiros disparando em cinco distâncias diferentes, 18 e 25m indoor e 50, 70 e 90m outdoor, foi visualizado que o músculo trapézio foi o mais rápido a ser recrutado, confirmando que os músculos da escápula iniciam a atividade antes que os demais músculos (elevação do braço e deslocamento para cima e medial da escapula). Estes dados foram confirmados no segundo experimento, verificando a variação da ancoragem e atividade muscular. No terceiro experimento, com arqueiros de vários desempenhos diferentes, este músculo apresentou a maior variabilidade nos arqueiros iniciantes, devido a sequência de ajustes posturais durante o lançamento da flecha. Isto não aconteceu com a mesma frequência em atletas de elite, mostrando que arqueiros de alto rendimento conseguem o recrutamento mais eficiente deste músculo, com menor oscilação. Isto pode estar relacionado com os nossos dados de desempenhos mais e menos elevados.

A análise do par agonista-antagonista RA-ML evidenciou nenhum efeito nas modulações de co-ativação Pré-APA, APA e APC do bloco, flechas e escores. Isto indica a constância na modulação ou variabilidade entre os arqueiros que inibe o 
surgimento do efeito. O mesmo se deu no índice de inibição reciproca, exceto da atividade do Multífido Lombar. Na análise de Vetor Codificado, a moda da coordenação foi Fase RA. Um dos fatores que podem explicar isto é o posicionamento do arqueiro no tiro, que aproxima as costelas da sínfise púbica, diminuindo a distância das inserções do Reto Abdominal o que pode aumentar sua atividade. No ensino do tiro, não é recomendado a posição de afastamento dessas inserções por dois fatores: o aumento da lordose lombar produz maior instabilidade no movimento e o olho do arqueiro fica desalinhado com a mira do arco, o que produz desempenho indesejável (ARCHERY, 1999; AXFORD, 2015; JOHNSON, 2014).

$\mathrm{Na}$ literatura ainda não há trabalhos que analisaram os músculos do tronco no tiro com arco. Tinazci (2011) observou em arqueiros de elite que o desempenho aumentava conforme a oscilação postural diminuía no sentido médio-lateral. Um arqueiro obteve pontuações mais altas conforme a oscilação para ântero-posterior era maior, enquanto um outro obtinha a melhor pontuação quando a oscilação da direita para esquerda diminuía. Existem dois posicionamentos do pé que os arqueiros costumam adotar no disparo, a posição Open Stance, onde o pé da frente fica ligeiramente atrás do pé posterior ao alvo, os pés e quadris ficam pouco inclinados em direção ao alvo; e a posição Square Stance, os pés e quadris ficam perpendiculares ao alvo, a maioria dos arqueiros preferem a primeira posição por se mais estável em campo aberto (ARCHERY, 1999; AXFORD, 2015; JOHNSON, 2014). A variabilidade intraarqueiros pode ocultar a análise global do conjunto. Cabe a futuros estudos a análise de outros músculos do tronco. Como o tiro com arco é uma atividade assimétrica, é interesse verificar o comportamento de músculos que agem assimetricamente como os Oblíquos internos/externos e Quadrado Lombar por exemplo.

No índice distal-proximal, o par agonista-agonista FSD-BBL não mudou ao longo dos blocos e flechas. $\mathrm{Na}$ análise de desempenho, diferenças ocorreram entre as fases do APA até o APC1 principalmente entre o escore 10 e 7. No APA, as modulações são de menor intensidade, e no APC, principalmente nos melhores desempenhos, ocorre a manutenção da co-ativação e diminuição nos piores. Na inibição recíproca, suprimindo a atividade do Bíceps Braquial, houve variação entre blocos, nos APA3 e APA2; nos blocos 4 e 5, e 2 comparado ao 8, 10 e 12. Nestes agonistas, não houve modulação temporal para flecha, e no desempenho ocorreram dois eventos com variação da inibição. No primeiro APA, APA3, o escore 10 foi maior que o 9 e 7, e no APC1, o escore 10 comparado ao 8 e 7 . Enquanto existem modulações de co-ativação agonista desde o pré-APA, a inibição ocorreu próxima a perturbação. No desempenho 
as modulações ocorrereram nos mesmos períodos. Na transição APA5-APA2, a moda foi Fase BBL e em APA2-APC1 foi Fase FSD. Para o desempenho, a mesma moda ocorreu. Inicialmente, a ativação é no sentido proximal, indo para o sentido distal de ativação no final do movimento, quando de fato é mais proeminente a presença de estratégia dos músculos do punho (MARTIN; SILER; HOFFMAN, 1990; ERTAN et al. 2003; SOYLU et al. 2006). Neste índice dos agonistas, destaca-se a relevância do músculo Bíceps Braquial na ação do movimento na preparação para o disparo, e depois a atividade muscular é estabilizada, enquanto o Flexor Superficial dos Dedos, com os demais flexores do punho e dos dedos, assuma maior protagonismo. Tendo em vista que uma atividade excessiva do Bíceps Braquial não é desejada para o desempenho motor (SHINOHARA, URABE, 2017; NISHIZONO et al. 1987).

O índice de co-ativação no par agonista-antagonista FSD-TBL não sofreu efeito de blocos no pré-APA (APA5 e APA4). Foi verificada maior no bloco 4 em relação 1, 8, 10-12 para o APA3, ou seja, a única oscilação temporal na análise de blocos ocorreu no primeiro ajuste postural antecipatório. Não houve mudança da co-ativação em APA2, APA1 e APC1. Nas flechas, essas diferenças não ocorreram, evidenciando consistência na ordem de disparo. Para a análise do desempenho, no APA3 maior no escore 10 em relação ao 8 e 7 e no APA2 e APC1, maior no 10 em comparação ao 7. Esta maior co-ativação pode garantir maior estabilidade nestes dois músculos que são importantes para obtenção de melhores desempenhos na modalidade, já que o Tríceps Braquial parece ter maior modulação em arqueiros de elite (SUWARGANDA et al. 2012). O comando de co-ativação C é um dos dois comandos básicos para a Hipótese do Ponto de Equilíbrio. Isso leva ao aumento na amplitude do ângulo articular, dentro do qual ambos os músculos agonistas e antagonistas são ativados sem mudar o ponto médio da amplitude o que aumenta a rigidez articular (LATASH, 2008). O índice de inibição recíproca, com supressão da atividade do Tríceps Braquial, mudou entre blocos e desempenho. No APA3, maior no bloco 4 em relação 1, 8,10-12. Na análise do desempenho, depois do pré-APA, todos os demais ajustes tiveram efeito na pontuação, no APA3 e APA2 no escore 10 em relação ao 8 e 7 e no APA1 com 9 em relação ao 8 e 7; e APC1:10 e 9 em comparação ao 7, ou seja existe comandos de inibição no APA1 para desempenho mas não de co-ativação.

Nos índices distal-proximal o par agonista-agonista constituído pelos FSD-BBL, não sofreu efeito ao longo da modulação de blocos, e flechas, ou seja, temporalmente a modulação de co-ativação é consistente neste índice. Contudo na análise de desempenho, diferenças foram evidenciadas no pré-APA, no primeiro APA e no APC; 
resumidamente no APA5 - maior no 10 comparado ao 7; APA4 - maior no escore 10 e 8 em comparação ao 7; APA3 maior no 10 em relação ao 7 e no primeiro ajuste compensatório - APC1 no escore 10 em comparação com 7 e 8 . Inicialmente as modulações são de menor intensidade, mas no ajuste compensatório, principalmente nos melhores desempenhos, ocorre a manutenção da modulação no índice, e diminuição de modulação nos piores. Na inibição recíproca - suprimindo a atividade do Bíceps Braquial - a análise evidenciou modificações de modulação no comando r- para bloco, a partir do primeiro APA - para os APA3 e APA2; nos blocos 4 e 5, e 2 comparado ao 8, 10 e 12; sem efeitos nos demais; ou seja só tem diferentes estratégias de modulação de inibição em 250-50ms antes da perturbação, nestes agonistas, não houve modulação temporal para flecha, e no desempenho ocorreram dois eventos onde estratégias de inibição podem ter sido utilizados, no primeiro APA - APA3, isto ficou evidenciado no escore 10 maior que o 9 e 7 e no primeiro ajuste compensatório - APC1 do escore 10 comparado ao 8 e 7. Ou seja, enquanto existem modulações de co-ativação agonista desde o pré-APA, as de inibição ocorrem já próximos a perturbação, no intuito de prever e estabilizar melhor o movimento para as análises temporais, no desempenho as modulações ocorrereram nos mesmos períodos. Já na análise de Vetor Codificado, evidenciou que na transição APA5-APA2, a moda foi de Fase do BBL, e do APA2APC1 de Fase do FSD. Para o desempenho, a mesma tendência foi observada moda, ou seja inicialmente a ativação é no sentido proximal, indo para o sentido distal de ativação no final do movimento, quando de fato é mais proeminente a presença de estratégia dos músculos do punho, em concordância com a literatura (MARTIN; SILER; HOFFMAN, 1990; ERTAN et al. 2003; SOYLU et al. 2006).

Resumidamente neste índice dos agonistas é possível que a relevância do músculo Bíceps Braquial na ação do movimento tenha importância no início da preparação para o disparo, e depois a atividade muscular é estabilizada, enquanto o Flexor Superficial dos Dedos, juntamente com demais flexores do punho e dos dedos, assumam um maior protagonismo. Tendo em vista que uma atividade excessiva do Bíceps Braquial não é desejada para o desempenho motor (SHINOHARA, URABE, 2017; NISHIZONO et al. 1987).

O índice de co-ativação: no par agonista-antagonista FSD-TBL; não evidenciou efeito na análise de blocos no pré-APA (APA5 e APA4) contudo uma observação pontual foi verificada maior no bloco 4 em relação 1, 8, 10-12 para o APA3, ou seja, a única oscilação temporal na análise de blocos ocorreu no primeiro ajuste postural 
antecipatório, após esta oscilação os comandos de modulação de blocos foi consistente no APA2, APA1 e APC1. Nas flechas esses efeitos não ocorreram, evidenciando alguma consistência na ordem de disparo. Para a análise do desempenho observações ocorreram após o pré-APA, no APA3 maior no escore 10 em relação ao 8 e 7 e no APA2 e APC1, maior no 10 em comparação ao 7. Sem modificações no APA1 (50ms antes e 50 após a queda do clicker). Esta maior co-ativação pode garantir maior estabilidade nestes dois músculos que são importantes para obtenção de melhores desempenhos na modalidade, já que o Tríceps Braquial parece ter maior modulação em arqueiros de elite (SUWARGANDA et al. 2012). O comando de co-ativação (c-) é um dos dois comandos básicos para uma articulação dentro da Hipótese do Ponto de Equilíbrio. Isso leva a um aumento na amplitude do ângulo articular, dentro do qual ambos os músculos agonistas e antagonistas são ativados sem uma mudança no ponto médio da amplitude o que aumenta a rigidez articular (LATASH, 2008). Contudo, no índice de inibição reciproca - com supressão da atividade do Tríceps Braquial, foram encontrados efeitos nos mesmos parâmetros de ajustes posturais para blocos e desempenho, como no primeiro ajuste depois do pré-APA, no APA3, maior no bloco 4 em relação 1, 8,10-12, sem diferenças temporais nas flechas; e na análise do desempenho, depois do pré-APA, todos os demais ajustes tiveram efeito na pontuação, no APA3 e APA2 no escore 10 em relação ao 8 e 7 e no APA1 com 9 em relação ao 8 e 7; e APC1:10 e 9 em comparação ao 7, ou seja existe comandos de inibição no APA1 para desempenho mas não de co-ativação.

A análise de Vetor Codificado no par FSD-TBL evidenciou que na transição APA5-APA2, a moda foi de Fase do TBL, e do APA2-APC1 de Fase do FSD, o que favorece uma ativação do punho e manutenção da atividade do cotovelo, ou seja temos uma sinergia no sentido proximal para distal na cadeia de movimento no momento do disparo de membros superiores. No desempenho as estratégias de coordenação foram diferentes em cada escore. Por exemplo, no escore 7 essa transição, ocorreu uma transição de fase do TBL para FSD no APA3-APA2, e 9 esta mesma transição de fases ocorreu no APA2-APA1. O escore 8 teve mais transições sem uma definição de parâmetro de movimento, já no escore 10 a moda foi observada somente de Fase do TBL. É possível que a essa de intensificação da atividade do TBL no escore 10 esteja relacionada com uma ação proximal do TBL, como extensor glenoumeral, o que reforça o papel deste músculo na obtenção de desempenho mais elevados (SUWARGANDA et al. 2012). 
Nota-se nos índices compostos com o FSD um contraste, por exemplo, no acoplamento com BBL o efeito na modulação ocorreu já no pré-APA e depos no primeiro ajuste postural antecipatório (APA5-APA4-APA3), já no acoplamento com o TBL os efeitos ocorreram nos ajustes de APA3-APA2, e ambos também foram modulados no APC1 - provavelmente pela ação do próprio FSD. Na análise de Fases de Coordenação, têm-se novamente um papel mais evidente do BBL no início dos ajustes, já o TBL alterna-se com o FSD na análise de desempenho. Estes fatores apresentados certamente aumentam a complexidade do entendimento de como arqueiros de alto desempenho realizam ajustes para obter melhores pontuações, incluindo fatores individuais que podem estar ocultos nas análises da população pesquisada (TINAZCI, 2011; SUWARGANDA et al., 2012, NISHIZONO et al., 1987)

No índice ED-BBL (antagonista e agonista) para co-ativação não foram encontrados efeitos na análise de blocos e flechas para transição (APA5-APC1); ou seja, não teve efeito na modulação os ajustes temporais neste índice. Contudo na análise de desempenho, com exceção do APA5 e APC1, todos os demais tiveram efeito; no APA4, maior no 10 que 7, APA3; maior no 10 em relação ao 8 e 7. APA2: maior no 10 comparado ao 7 e 8, e 9 maior que 7. APA1 maior no 9 e 8. Modulações evidentes foram observadas no período de 350ms antes da queda do clicker até 50ms após a queda do mesmo, conferindo alguma estabilidade no período pré-disparo. Já no índice de inibição recíproca, com supressão da atividade antagonista, foram encontrados efeitos no primeiro ajuste postural antecipatório (250-150ms antes da perturbação), APA3, maior no bloco 5 em comparação ao 2, 6-12; evidenciando um comando de inibição - a ativação recíproca diminui a atividade do músculo antagonista enquanto aumenta a do agonista (LATASH \& ZATSIORSKY, 2015), não houve efeito nas flechas; ou seja não tem modulação pronunciada na ordem do tiro disparado, já no desempenho diferenças foram encontradas com comandos r- no fim do pré-APA (APA4) até o segundo ajuste antecipatório (APA2), numa janela de 350ms até 50ms antes da perturbação, ou seja enquanto estratégias de co-ativação foram mais consistentes até a queda do clicker as de inibição cessaram antecipadamente. No APA4, foi observado mais modulação no 10 comparado ao 7; APA3; maior no 10 comparado ao 9,8 e 7; APA2; novamente maior no 10 em comparação com o 7.

$\mathrm{Na}$ análise de Vetor Codificado para este índice ED-BBL, a moda de transição no APA5-APA3 é de Fase do BBL com modificação para Fase de ED do APA3 até o APC1, na ordem proximal para distal. Contudo para o desempenho, os padrões de 
coordenação em cada escore foram totalmente heterogêneos, cada escore teve uma moda de coordenação específica, destaca-se a presença da moda de coordenação Em Fase em três escores analisados, em fases distintas, o que torna indefinido que tipo de coordenação prevalece com maior frequência neste índice na análise de desempenho.

Para a co-ativação no índice ED-TBL (antagonistas), não foram encontrados efeitos na análise de blocos para os ajustes; APA5, APA4, APA2, APA1 e APC1; somente no primeiro ajuste postural pós pré-APA (APA3), houve efeito maior no bloco 4 em comparação com o 1,2,8,10-12, ou seja os antagonistas modulam de modo diferente na análise de blocos somente no período de 250-150ms antes da queda do clicker, nas flechas não foi observado efeito. Nos escores diferenças foram visualizadas do APA3 ao APC1. Para o APA3, maior no escore 10 em relação ao 8 e 7 . APA2; 10 maior que o escore 8 e 7, e 9 maior que 7. APA1: no escore 9 e 7. APC 1, no 10 maior que o escore 7 foram evidenciadas, ou seja existe muitas modulações antagonistas no período de $250 \mathrm{~ms}$ antes da queda do clicker, até $150 \mathrm{~ms}$ após a queda do mesmo, o que parece consistente com os dados de modulação que encontra-se na literatura para estes dois músculos neste período (TINAZCI, 2011; SUWARGANDA et al., 2012, NISHIZONO et al., 1987)

$\mathrm{Na}$ análise do índice de inibição recíproca ED-TBL, houve efeito do bloco em APA3 e sem efeito nas flechas. Há variação da inibição no primeiro ajuste postural antecipatório. No desempenho, foi observado efeito no APA3: maior no 10 comparado ao 8 e 7; APA2: 10 comparado ao 9,8 e 7; sem efeito no APA1, mas com efeito no APC1 entre 10 e 7, ou seja é essa ação destes antagonistas não é diferente no desempenho no período do APA1 onde parece ser mais necessário que estes músculos desempenhem um papel de co-ativação.

Na análise de Vetor Codificado, a moda de transição no APA5-APA3 é de Fase do TBL com modificação para Fase de ED do APA3 até o APC1. Seguindo a tendência temporal observada em todos os demais pares de análise proximal-distal, com a ativação aumentado no sentido dos músculos do cotovelo para os do punho. Contudo, para o desempenho, os padrões de coordenação em cada escore foi novamente heterogêneo; o escore 7 e 9 teve coordenação semelhante a moda geral das flechas disparadas; no escore 8 e 10 a coordenação predominante foi a de Fase do TBL com exceção do APA2-APA1; e APA3-APA2 respectivamente. É possível nos pares FSD-BBL, FSDTBL ED-BBL e ED-TBL podemos ter coordenação semelhante em escores diferentes, como quando o arqueiro atira no escore 10 seguido do 8, ou vice-versa, como qualquer 
outra combinação, tornando mais complexa esta análise quando é feito o acoplamento de músculos que não são respectivamente agonista e antagonista da mesma articulação.

Nos pares que compuseram o ED, no acoplamento com BBL, houveram efeitos na modulação de três ajustes pré-clicker (APA3-APA1), no acoplamento TBL ocorreram ajustes no APA3- APC1. As observações sobre os índices proximal-distal entre cotovelo e punho apresentam relações similares no pré e imediatamente pósclicker, na análise dos blocos elas foram pontuais principalmente no APA3, nos escores a tendência foi a de os músculos agonistas serem mais solicitados nos momentos inicias, com estabilização no momento APA5-APA3 (500-250ms pré queda do clicker) e nos APC1 (50-150 pós queda do clicker), enquanto os antagonistas são mais solicitados da metade da janela no desempenho até os ajustes compensatórios - APA3-APC1 (250ms pré clicker - 150ms pós clicker) - esses dados são parcialmente corroborados com a literatura, que podem evidenciar co-ativação de músculos antagonista após a queda do clicker, principalmente antagonista, com diminuição da atividade muscular após 200ms a queda do clicker - contudo a nova evidência é a de que diferentes níveis de coativação, inibição reciproca, e fase de coordenação ocorrem quando análises de desempenho - por pontuação - são realizados (ERTAN et al., 2003, 2011; NISHIZONO et al., 1987; KOLAYIŞ, ERTAN, 2016).

No índice glenoumeral-escápula antagonista PMC-SAD de co-ativação não houve efeito para a análise dos blocos e flechas em todos os ajustes analisados de APA5-APC1 ou seja não ocorre uma modulação temporal tão discrepante neste índice; já no desempenho os efeitos ocorreram a partir do APA2; entre o 10 comparado 9 e 7, e nos ajustes APA1 e APC1: 10 e 9 em comparação com o 7; é necessária uma maior coativação entre esses dois músculos nestes ajustes para garantir maior estabilidade no complexo escápula-úmero e aumentar o desempenho; isso fica evidente depois que notamos que nenhum efeito foi observado no índice de inibição reciproca para blocos, flechas e escores.

Para a análise de vetor codificado, observamos no par PMC-SAD que a moda de coordenação foi de Fase de SAD para a transição de APA5-APA3 e Fase de PMC de APA3-APC1. Nos escores a tendência foi a mesma, com exceção do escore 10 na transição APA5-APA4, Em Fase; e APA4-APA3 onde o resultado de coordenação foi bimodal com Fase de SAD e Fase PMC, ou seja, inicialmente ocorre maior estabilização na escápula para depois aumentar a atividade nos músculos da articulação glenoumeral. 
A literatura evidencia que os músculos periescapulares e do manguito rotador acionam mais rapidamente que músculos glenoumerais mais potentes como o Peitoral Maior em gestos esportivos no intuito de estabilizar o complexo do ombro - e ainda na presença de lesões esses músculos (periescapulares e do manguito rotador), acionam mais tardiamente, diminuindo a estabilidade do complexo, e também reduzindo a potência do movimento (HORSLEY; HERRINGTON; ROLF, 2010; HERRINGTON \& HORSLEY 2009), no presente estudo a análise de Vetor Codificado indicou de fato esta tendência de ativação no sentido de fase dos periescapulares para fase dos glenoumerais no par antagonista PMC-SAD, contudo o nível da modulação de coativação foi mais elevado nos escores mais altos, o que pode ser um indício de que existe a necessidade desta maior ativação para atingir maior desempenho.

No índice de co-ativação e de inibição reciproca; na glenoumeral-escápula agonista (GD-TS) não foram encontradas diferenças estatisticamente significativas na análise dos blocos, flechas e escores; apesar da variabilidade de resultados, principalmente no desempenho. Análise de vetor codificado: a moda de coordenação neste par de músculos foi de Fase do TS durante todas as transições. Nos escores a tendência foi a mesma, com todos os escores, no acoplamento GD-TS apresentarem Fase do TS. A importância do músculo Trapézio Superior é maior que a do Grande Dorsal, no tiro com arco, estando mais relacionado ao desempenho obtido em alguns casos (SUWARGANDA et al., 2012).

O músculo Grande Dorsal pode não ser tão efetivo na posição da puxada realizada no tiro do arco, que se situa entre 105-95 graus de abdução da articulação glenoumeral em arqueiros de alto rendimento (SHINOHARA \& URABE, 2017), este músculo é mais potente como adutor e extensor (SIGNORILE, ZINK, SZWED, 2002) do que como abdutor horizontal desta articulação, além disto ele divide a ação da puxada com outros músculos periescapulares e glenoumerais como, respectivamente, o trapézio e rombóides e o deltóide posterior, redondo maior, e tríceps cabeça longa, conferindo maior redundância a este movimento, o que pode exemplificar a sua ação dimínuida no tiro com arco (SOYLU et al. 2006).

O índice de co-ativação envolvendo o Serrátil Anterior de ambos os lados (SAD$\mathrm{SAnD}$ ); evidenciou uma pronunciada modulação, desde o pré-APA até o penúltimo APA, na análise de blocos; no APA5 e APA4, foram encontrados efeitos com maior modulação no bloco 5 em comparação com os blocos de 1-4,6-11, no APA3, maior no bloco 5 em comparação com 12 e APA2; no 5 comparado 1,2,4 e 7; no APA1 e APC1 não foi encontrado efeito, é destacada a necessidade de ativação destes músculos 
periescapulares, o que implica nessa modulação mais pronunciada desde 500ms pré perturbação. Não foi encontrado efeito na ordem das flechas. $\mathrm{Na}$ análise dos escores também foram evidenciados efeitos desde o pré-APA, no APA5, maior no escore 10 comparado ao 9; depois estabiliza-se a modulação, com nenhum efeito no APA4 e APA3; e voltando a modular diferente no desempenho no APA 2: com maior modulação no 10 comparado ao 7. APA 1, maior no 9 comparado ao 8 e 7 e no APC 1 , com 10 e 9 mais elevados que 7 e 8 . Contudo é interessante que os índices de inibição recíproca evidenciaram resultados similares, na análise de blocos; com resultados idênticos do APA5 ao APA2; e no APA1 e APC1 não foi observado efeito, nenhum efeito para flechas. A mesma tendência de comando de co-ativação foi evidenciada no comando de inibição nos escores. Na análise de Vetor Codificado a moda de coordenação na transição APA5-APA4 até APA2-APA1 foi de Fase do SAD, com modificação no APA1-APC1 para Fase do SAnD; as modas neste foram equivalentes entre Fase do SAD e Fase do SAnD. Na análise de escores foi observada uma alternância de fase nas transições analisadas entre Fase SAD e Fase SAnD.

Estes músculos desempenham papéis antagônicos nesta modalidade, enquanto o lado que traciona a corda (dominante) age como antagonista, o lado que segura o arco (não-dominante) age como agonista na abdução glenoumeral; diferenças significativas foram observadas tanto bloco-a-bloco, como no desempenho, assim como na análise de Vetor codificado as modas foram de Fase de SAD e de SAnD, o que impossibilita a distinção de importância entre eles, pela heterogeneidade de estratégias utilizadas. O músculo Serrátil Anterior é um dos principais componentes do ritmo-escapuloumeral, uma atividade insatisfatória dele reduz a rotação lateral e protração da escápula, isto possibilita que a cabeça do úmero seja translacionada no sentido anterior e superior, desta forma uma atividade retardada ou reduzida deste músculo pode acarretar num problema que impossibilita o complexo do ombro de produzir um freio excêntrico eficiente para atividades que demandam altíssima aceleração (ALLEGRUCCI et al., 1995; LUDEWIG \& COOK, 2000), como por exemplo na queda do clicker até a completa efetuação do disparo no tiro com arco (NISHIZONO et al., 1987).

No lado que segura o arco foi analisado o par de músculos SAnD-DPnD, no índice de co-ativação, houve efeito para blocos no primeiro pré-APA somente - APA5 com maior modulação no 5 comparado com 1,2,7,10-12; nos demais essas diferenças não foram detectadas (APA4-APC1). Isto é um indicativo que estes músculos, trabalhando em isometria segurando o arco modulam no pré-APA, e depois mantém constante a ativação nos demais ajustes como também na análise de ordem a partir do 
disparo de flechas. Já nos escores ocorreram efeitos no pré-APA; APA5 - maior coativação no escore 10 comparado ao 7 e 9; APA 4, maior modulação no $10 \mathrm{em}$ comparação ao 9; sem diferenças dos ajustes APA3-APA1; ou seja, existe uma coativação constante até a 50ms pós queda do clicker, já nos ajustes compensatórios (50$150 \mathrm{~ms}$ ), momento do dito follow-through do arco, quando ele gira em sentido horário, existe modulação na pontuação 9 em comparação ao 8. Isto é um indicativo que alguns arqueiros podem procurar alguma estratégia de co-ativação para estabilizar melhor a oscilação do disparo.

Para o comando de inibição reciproca, no par que segura o arco, só ocorreram modulações no pré-APA para análise de blocos e escores. Para bloco no APA5, maior modulação no 5 comparado com 1,2,7,10-12; nos demais essas diferenças não foram detectadas (APA4-APC1). Nenhum efeito na ordem das flechas. E novamente nos escores, só foi diferente a modulação no pré-APA; APA5 e APA4; com maior modulação no escore 10 comparado ao 9; sem diferenças para os demais ajustes na análise de desempenhos (APA3-APC1). Ou seja, enquanto no pré-APA, os arqueiros podem procurar estratégias de modulação que variam tanto de inibição, como coativação, no ajuste postural compensatório (APC1), só são detectadas diferenças para co-ativação no desempenho, o que pode garantir alguma estabilidade diferenciada frente a perturbação do movimento de rotação do arco (follow-through), momento assimétrico, onde é necessário a manutenção de uma tensão no braço que segura o arco seguida de um relaxamento progressivo (AXFORD, 2015).

$\mathrm{Na}$ análise de Vetor Codificado a moda de coordenação na transição APA5APA3 foi de Fase do DPnD, com modificação no APA3-APC1 para Fase do SAnD, o que está em consonância com o preceito técnico da modalidade, pois o $\mathrm{DPnD}$ é mais potente, e garante tensão segurando o arco, conforme o disparo da flecha se aproxima, uma ativação dos músculos da escápula, pode ser necessária para garantir estabilidade do complexo perante a perturbação, na análise dos escores essa tendência também foi observada, com algumas oscilações pontuais do escore 8 nas transições de APA3-APA1 e do escore 10 no APA2-APA1, onde a coordenação predominante foi de Fase do DPnD, no APA1-APC1, sem excessão foi utilizada coordenação de Fase do SAnD. De modo geral a análise de músculos no lado que segura o arco é pouco evidenciada na literatura, contudo também é de suma importância, Ertan (2009) verificou que a atividade muscular dos extensores dos dedos nos arqueiros de elite foi significativamente maior que iniciantes. No presente estudo somente os músculos Deltóide Posterior e Serrátil Anterior do lado não-dominante foram analisados, nota-se 
variações significativas bloco-a-bloco e no desempenho no período Pré-APA, que ocorre entre 500-250ms antes da queda do clicker, possivelmente pela posição em isometria que deve ser mantida antes da efetuação do disparo, verifica-se também juntamente com a análise de Vetor Codificado que o período subsequente de APA3APC1 existem mais modificações na coordenação entre Fase de SAnD e Fase de DPnD, estabilizando no período final - ajuste compensatório (50-150ms após a queda do clicker) - com Fase do SAnD, possivelmente porque é necessária uma estabilização sutil do arco o que corresponde com seu papel estabilizatório anteriormente citado (LUDEWIG \& COOK, 2000).

$\mathrm{Na}$ análise do último par (GD-DPnD), para co-ativação não ficou evidenciado a presença de qualquer efeito temporal para blocos e flechas nos ajustes analisados (APA5-APC1); nos escores diferenças pontuais ocorreram no primeiro pré-APA (500400ms antes da queda do clicker) no APA5 com maior modulação no escore $10 \mathrm{em}$ comparação ao 9 e 7; estabiliza a modulação no período até $250 \mathrm{~ms}$ antes da queda do clicker, depois sofre novo efeito de modulação no período de 250-150ms antes da queda (APA3) no escore 10 em comparação ao 9; novamente estabilizando-se no restante do movimento. É possível que essas modulações ocorram em maior frequência pela ação do Deltóide Posterior não dominante, que possui uma ação peculiar no tiro com arco. Este músculo não é um abdutor glenoumeral potente (ACKLAND et al., 2008), contudo possui ação agonista como extensor horizontal da mesma articulação, e curiosamente é mais ativado no braço que segura o arco do que o traciona a corda (KOLAYIŞ, ERTAN, 2016), um motivo importante para que isto aconteça está relacionado com a linha de força - o arqueiro precisa posicionar a corda e o arco mais próximo do seu corpo, aproximando as escápulas, isso produz um movimento no braço que segura o arco de isometria em abdução com extensão horizontal desta articulação, potencializando a atividade do Deltóide Posterior no movimento (ARCHERY, 1999; JOHNSON, 2014), o que nos ajuda a exemplificar tais comandos diferenciados de coativação nos arqueiros.

Os comandos de inibição reciproca, ocorreram pontualmente nas mesmas análises pré-APA e no primeiro APA para escores, não sendo evidenciado efeito para blocos e flechas nos ajustes analisados (APA5-APC1). Nos ajustes iniciais; APA5: 10 em comparação ao 9 e 7; e APA3: 10 em comparação ao 9; sem diferenças no APA4 e APA2-APC1. Indicando esses ajustes singulares, principalmente pelas estratégias do músculo Deltóide posterior, anteriormente explicitado sobre sua ação no tiro com arco, isto corrobora com a análise de Vetor Codificado, onde a a moda na transição de todos 
os ajustes analisados no total de flechas disparadas, e na análise individual de desempenho nos escores, a coordenação predominante foi de Fase do DPnD. A evidência deste par também corrobora com a perspectiva anterior dos demais que compuseram o músculo Grande Dorsal (GD-PMC e GD-TS), nos quais as modulações ocorrem ou no componente antagonista, ou escapular ou contralateral. Destaca-se vários fatores para que a atividade deste músculo (Grande Dorsal) seja menor do que se esperava para um agonista na puxada da corda; como por exemplo, a redundância nos músculos que tracionam a corda, como mencionado anteriormente (SOLYU et al., 2006); e ele também ser mais eficiente como adutor e extensor, do que abdutor horizontal, além disso sua produção de torque mais efetiva ocorre abaixo de $70^{\circ}$ de abdução da articulação glenoumeral, na sua porção superior - cujas fibras são mais horizontais, e $10^{\circ}$ nas fibras médias e inferiores (ACKLAND et al., 2008).

Todos os pares analisados neste estudo, a partir da observação de co-ativação e de inibição recíproca juntamente com a análise de Vetor Codificado, são uma pequena amostra de casos que devem ser levados em consideração na análise do desempenho em arqueiros de elite, outros aspectos também são relevantes no rendimento destes arqueiros, que não foram analisados no presente estudo, como a análise cinemática; no momento da puxada da flecha o complexo do ombro precisa ser ativado para mantê-la alinhada ao alvo. Pequenas flutuações podem ser identificadas na eletromiografia (tremor) - Lin et al. (2010) analisaram este fator em segmentos; no ombro: Deltóide médio, Bíceps Braquial, Infra-Espinhal e Redondo menor; e escápula: Trapézio superior, Serrátil Anterior, Romboide maior e Latíssimo do dorso em três posições articulares: a $90^{\circ}, 100^{\circ}$ e $110^{\circ}$. A exigência dos músculos do ombro foi maior, e o poder do espectro do tremor foi significativamente maior em 100 e 110 graus quando comparado a 90 graus, este último ângulo parece ser o mais eficiente para efetuar o tiro pelo menor número de flutuações e força muscular exigidas. Shinohara \& Urabe (2017), corroboram com essa perspectiva, identificando que arqueiros de elite atiram com angulação glenoumeral entre 95-105 graus; enquanto arqueiros de nível inferior atiram com ela variando de 102-112 graus - isto parece interessante de ser levado em consideração, se quando os arqueiros de elite atiram com menor ou maior eficiência o ângulo utilizado difere ou não.

Outras questões que parecem ter relação com o desempenho são o tempo de preparação para o tiro, e o tempo de reação ao clicker; no primeiro caso, Takai; Kubo; Araki (2012) corroboraram com a perspectiva que arqueiros que conseguem as primeiras colocações gastam menor tempo no preparo do tiro que os que não sobem ao 
pódio, em Tinazci (2011), foi também observado que em pontuações mais elevadas os arqueiros reagiam mais rapidamente ao estímulo do clicker, mais recentemente Taha et al., (2016), verificaram que em pontuações mais altas o arqueiro utiliza um menor tempo mirando no alvo. É possível que isso se traduza na atividade muscular - Ertan et al. (2003), por exemplo, encontraram que logo após a queda do "clicker" os arqueiros de elite, novatos e não arqueiros realizavam uma contração ativa de extensores dos dedos, contudo arqueiros de elite acionaram esta musculatura no pico a 100ms após a queda, seguido de um rápido relaxamento, novatos à 200ms e não arqueiros a 300ms, isso corrobora com esta possibilidade de estratégia de co-ativação seguida de inibição reciproca.

Os resultados obtidos pelo presente estudo evidenciam aspectos da análise do desempenho esportivo na determinação do rendimento, entre pontuações melhores e piores, contudo destaca-se a quantificação dos dados a partir das técnicas foram provenientes da Hipótese de Ponto de Equilíbrio e dos Sistemas Dinâmicos, para respectivamente a análise de co-ativação/inibição recíproca e coordenação muscular. Na análise de co-ativação e inibição recíproca, algumas técnicas podem ser descritas na literatura, além da que foi utilizada no presente estudo baseada no trabalho de Slijper e Latash (2002), que pode ser considerada uma limitação deste trabalho. Ervilha, GravenNielsen e Duarte (2012) sugerem outras duas utilizadas na literatura; que se baseiam simplificadamente na razão da atividade do músculo antagonista sobre o agonista, outras técnicas sugeridas são as de correlação como a correlação cruzada e análise de coerência (NELSON-WONG et al, 2008; WINTER, 2008). Estes aspectos evidenciam a dificuldade de unificar um método eficiente para avaliação destes comandos neuromusculares, pois cada método não é excludente do outro, mas podem responder questões diferentes, além disso, a prudência deve ser levada em consideração como reitera Ervilha, Graven-Nielsen e Duarte (2012), no intuito de evitar interpretações claudicantes e fastidiosas, o que pode ser considerado um fator limitante na técnica de análise de co-ativação/inibição recíproca. Além disto, destacamos outra limitação que é a anexação de eletrodos na pele do arqueiro, o que causa inevitável efeito retroativo que pode influenciar o desempenho obtido. Contudo é inegável que foram identificados no presente estudo pares de músculos que foram coativados ou inibidos reciprocamente de modo diferente conforme se sucedia a sequência de disparo das flechas e no escore que estes arqueiros obtinham. Destacamos aqui, particularmente, os músculos FSD e TBL, que foram coativados com maior frequência em escores mais elevados, assim como a 
estratégia de diminuição de ativação do TS pode estar também relacionada com a obtenção de escores mais elevados.

Na mesma linha, temos algumas das técnicas de análise coordenativa dentro dos Sistemas Dinâmicos, nos quais se destaca as de fase relativa (Fase Relativa Discreta; Fase Relativa Discreta Multifrequencial; Fase Relativa Contínua; Fase de Fourier; Transformação de Hilbert) e a de Vetor Codificado; com seus prós e contras como mostra-nos Van Emmerik; Miller; Hamill, (2013); citando alguns aspectos que podem influenciar na técnica de análise como o sinal aplicado (sinusoidal e não-sinusoidal), a sensibilidade, a normalização, e também a perda de informações na transição de fases ou na medição da variabilidade, e novamente fazemos a mesma análise que nenhum deles exclui o outro, mas busca responder questões diferentes. Funcionalmente a análise de Vetor Codificado é mais vantajosa, pois permite uma melhor transição para a sua utilização na técnica eletromiográfica, pela possibilidade de construção de fases. Foram descritos anteriormente, com simplificações. Cabe ressaltar que até o presente momento nenhum trabalho aplicou nos sinais eletromiografícos a análise de Vetor Codificado, sendo este o primeiro que temos conhecimento. Desta maneira as informações obtidas na transição de ajustes nessa técnica juntamente com as de co-ativação/inibição reciproca, ajuda-nos a ter um panorama maior da análise do movimento humano e suas complexidades.

Destacam-se, além das convergências e divergências das técnicas de análise, algumas limitações do presente estudo como: a coleta ter sido realizada em dia único, em campo aberto com possibilidade de interferências climáticas, a análise somente da eletromiografia como parâmetros de desempenho, pois o sinal eletromiográfico possui variabilidade no arqueiro e entre os arqueiros, a amostra - apesar de ser a seleção nacional - ser mista, homens e mulheres, podendo influenciar nas análises dos músculos do tronco e membros superiores, além do efeito retroativo dos eletrodos anexados no corpo. A construção dos pares musculares revelou complexidade na detecção e interpretação dos resultados, pois esses músculos, que não pertencem a ações agônicas e antagônicas na mesma articulação, necessitam de uma análise cinemática para ser destacada sua ação como agonista ou antagonista. Estes fatores limitantes devem ser levados em consideração nos estudos futuros. 


\section{CONCLUSÃO}

Destacam-se, nas principais evidências do presente estudo, os músculos que sofreram maior efeito de modulações temporais ou de desempenho. Por exemplo, o músculo FSD, foi bastante modulável e possuem certas particularidades que ainda não tinham sido evidentes em outros trabalhos, o presente estudo evidencia que os arqueiros utilizam variadas estratégias coordenativas, que vai de encontro com a premissa que existe somente uma ativação do ED com inibição do FSD em arqueiros de elite. O músculo TBL possui relação com o desempenho obtido em escores mais elevados, enquanto o BBL é mais ativado em escores inferiores. O PMC possui uma ação estabilizadora no controle do movimento de coativação, enquando o GD é secundário na glenoumeral pela posição do arqueiro utilizada. As estratégias nos músculos da escápula também são bastante moduláveis, é possível que uma estratégia que aumente o TPS e mantenha ou iniba o SAD esteja relacionada com desempenhos inferiores, enquanto maior coativação do SAD parece melhor estabilizar a escápula garantindo escores superiores. No tronco não foram encontrados efeitos no RA e ML, é possível que esses músculos tenham fatores individuais heterogêneos que não permitam distinções em âmbito global de análise. Os músculos analisados no lado que segura o arco parecem ter importância significativa, e poucos estudo os contemplam, eles modulam diferentemente com coativação iniciada pela escápula e finalizada no ombro.

É fato notório que arqueiros de elite podem utilizar diferentes níveis de atividade eletromiográfica para atingir melhores pontuações, a novidade encontra-se na seguinte questão, como as abordagens de controle motor nos ajudam a identificar em qual tipo de fase ficou determinado par de músculos durante a execução do tiro, e na intensidade encontrada no nível de coativação e inibição reciproca utilizados temporalmente (blocoa-bloco) e no desempenho, apesar dos resultados técnico-depedentes. Nisto evidencia-se pelo presente estudo que na maior parte dos pares musculares analisados alguma diferença foi encontrada nestes aspectos, acrescentando informações novas sobre a coordenação do movimento humano e a variabilidade de estratégias que atletas podem utilizar na obtenção de desempenho de menor ou maior escala. 


\section{REFERÊNCIAS BIBLIOGRÁFICAS}

ACKLAND, D. C. et al. Moment arms of the muscles crossing the anatomical shoulder. Journal of Anatomy, v. 213, n. 4, p. 383-390, 2008.

ALLEGRUCCI, M. et al. Shoulder kinesthesia in healthy unilateral athletes participating in upper extremity sports. Journal of Orthopaedic \& Sports Physical Therapy, v. 21, n. 4, p. 220-226, 1995.

ALIBERTI, S. Influência da síndrome da dor patelofemoral nos movimentos e coordenação do joelho, tornozelo e pé no descer escada e efeitos imediatos de uma intervenção. 2015. Tese (Doutorado em Biodinâmica do Movimento Humano) - Escola de Educação Física e Esporte, Universidade de São Paulo, São Paulo, 2015.

APEX HUNTING. Recurve Bow figure clicker. 2019 (Https:// https://www.apexhunting.com.au/p/recurve-bow-clicker/TP-TR132)

ARCHERS, Balbardie. Archer's reference guide (recurve). 1999.

ARISTOTLE "The incessu animalium" in Aristotle, Ross, W. D. Smith, J.A. The works of Aristotle translated into English. 1928.

ARUIN, A. S.; LATASH, M. L. Directional specificity of postural muscles in feedforward postural reactions during fast voluntary arm movements. Experimental brain research, v. 103, n. 2, p. 323-332, 1995.

ARUTYUNYAN, G. A.; GURFINKEL, V. S.; MIRSKII, M. L. Investigation of aiming at a target. Biophysics, v. 13, n. 3, p. 642-645, 1968.

ARUTYUNYAN, G. A.; GURFINKEL, V. S.; MIRSKII, M. L. Organization of movements on execution by man of an exact postural task. Biophysics, v. 14, n. 6, p. 1162-+, 1969.

AXFORD, R. Archery Anatomy: Archery Anatomy. Souvenir Press, 2015.

BARTLETT, R.; WHEAT, J.; ROBINS, M. Is movement variability important for sports biomechanists? Sports biomechanics, v. 6, n. 2, p. 224-243, 2007.

BAUER, HU.; SCHÖLLHORN, W. Self-organizing maps for the analysis of complex movement patterns. Neural Processing Letters, v. 5, n. 3, p. 193-199, 1997.

BERNSTEIN, N. A. The coordination and regulation of movements. 1. ed. [s.l.] Pergamon Press, 1967. p. 1-196.

BERNSTEIN, N. A.; LATASH, M. L.; TURVEY, M. Dexterity and its development. Taylor \& Francis. 1996. P. 3-244

CHANG, R.; VAN EMMERIK, R.; HAMILL, J. Quantifying rearfoot-forefoot coordination in human walking. Journal of Biomechanics, v. 41, n. 14, p. 3101-3105, 2008 .

CLARYS, J. P. et al. Muscular activity of different shooting distances, different release techniques, and different performance levels, with and without stabilizers, in target archery. Journal of Sports Sciences, v. 8, n. 3, p. 235-257, 1990. 
CRAM, J.; KASMAN, G.; HOLTZ, J. Introduction to surface electromyography. 1. ed. Aspen: Gaithersburg, Md. : Aspen Publishers, 1998.

EDELMANN-NUSSER, J.; GOLLHOFER, A. Coordinative aspects of archery-an approach using surface-electromyography. In: ISBS-Conference Proceedings Archive. 1998.

EKSTROM, R. A. et al. Surface electromyographic analysis of exercises for the trapezius and serratus anterior muscles. Journal of Orthopaedic \& Sports Physical Therapy, v. 33, n. 5, p. 247-258, 2003.

EKSTROM, R. A. et al. Normalization procedures using maximum voluntary isometric contractions for the serratus anterior and trapezius muscles during surface EMG analysis. Journal of Electromyography and Kinesiology, v. 15, n. 4, p. 418-428, 2005 .

ERTAN, H. et al. Activation patterns in forearm muscles during archery shooting. Human Movement Science, v. 22, n. 1, p. 37-45, 2003.

Muscular activation patterns of the bow arm in recurve archery. Journal of Science and Medicine in Sport, v. 12, n. 3, p. 357-360, 2009.

Individual Variation of Bowstring Release in High Level Archery: A Comparative Case Study. Human Movement, v. 12, n. 3, p. 273-276, 2011.

ERVILHA, U. F.; GRAVEN-NIELSEN, T.; DUARTE, M. A simple test of muscle coactivation estimation using electromyography. Brazilian Journal of Medical and Biological Research, v. 45, n. 10, p. 977-981, 2012.

FERBER, R.; DAVIS, I. M.; WILLIAMS III, D. S. Effect of foot orthotics on rearfoot and tibia joint coupling patterns and variability. Journal of biomechanics, v. 38, n. 3, p. 477-483, 2005.

FLEISIG, Glenn S. et al. Kinetics of baseball pitching with implications about injury mechanisms. The American journal of sports medicine, v. 23, n. 2, p. 233-239, 1995.

FREEMAN, $\mathrm{H}$. On the encoding of arbitrary geometric configurations. IRE Transactions on Electronic Computers, n. 2, p. 260-268, 1961.

GELFAND, I. M.; LATASH, Mark L. On the problem of adequate language in biology. Em: Latash, M.L. (Ed.), Progress in Motor Control, Structure-Function Relations in Voluntary Movement, vol. 2. Human Kinetics, Urbana, IL, p. 209-228. 1998

GLAZIER, P. S; WHEAT, J. S.; PEASE, D. L.; BARTLETT, R. The interface of biomechanics and motor control. In DAVIDS, K; BENNETT, S; NEWELL, K M. Movement system variability. Human kinetics, v. 1, p. 49-69, 2006.

GRAUBNER, R.; NIXDORF, E.. Biomechanical analysis of the sprint and hurdles events at the 2009 IAAF World Championships in Athletics. New studies in athletics, v. 26, n. 1/2, p. 19-53, 2011.

GUIGON, E.; BARADUC, P.; DESMURGET, M. Optimality, stochasticity, and variability in motor behavior. Journal of computational neuroscience, v. 24, n. 1, p. 57-68, 2008. 

control, v. 20, p. 478-502, 2011.

HAMILL, J.; HADDAD, J. M.; MCDERMOTT, W. J. Issues in quantifying variability from a dynamical systems perspective. Journal of Applied Biomechanics, v. 16, n. 4, p. 407-418, 2000.

KNUTZEN, K. M. Biomechanical basis of human movement. Lippincott Williams \& Wilkins, 2014.

HEIDERSCHEIT, B. C.; HAMILL, J.; VAN EMMERIK, R. E. A. Variability of stride characteristics and joint coordination among individuals with unilateral patellofemoral pain. Journal of applied biomechanics, v. 18, n. 2, p. 110-121, 2002.

HENNEMAN, E.; SOMJEN, G.; CARPENTER, D. O. Excitability and inhibitibility of motoneurons of different sizes. Journal of neurophysiology, v. 28, n. 3, p. 599-620, 1965.

HENNESSY, M. P.; PARKER, A. W. Electromyography of arrow release in archery. Electromyography and clinical neurophysiology, v. 30, n. 1, p. 7-17, 1990.

HERRINGTON, L.; HORSLEY, I. Electromyographic analysis of selected shoulder muscles during a rugby football tackle. BMC sports science, medicine and rehabilitation, v. 1, n. 1, p. 10, 2009.

HERMENS, H. J. et al. Development of recommendations for SEMG sensors and sensor placement procedures. Journal of Electromyography and Kinesiology, v. 10, n. 5, p. 361-374, 2000.

HORSLEY, I. G.; HERRINGTON, L. C.; ROLF, C. Does a SLAP lesion affect shoulder muscle recruitment as measured by EMG activity during a rugby tackle? Journal of orthopaedic surgery and research, v. 5, n. 1, p. 12, 2010.

JOHNSON, Teresa. Archery Fundamentals. Human Kinetics, 2014.

KAPANDJI, A. I. Fisiologia articular: esquemas comentados de mecânica humana, v. 1: 1. ombro, 2. cotovelo, 3. prono-supinação, 4. punho, 5. mão. 2007.

KOLAYIŞ, İ. E.; ERTAN, H. Differences in activation patterns of shoulder girdle muscles in recurve archers. Pamukkale Journal of Sport Sciences, v. 7, n. 1, p. 25-34, 2016.

LATASH, Mark L. Neurophysiological basis of movement. Human Kinetics, 2008. Synergy. Oxford University Press, 2008

; ZATSIORSKY, Vladimir. Biomechanics and motor control: defining central concepts. Academic Press, 2015.

LEROYER, P.; VAN HOECKE, J.; HELAL, J. N. Biomechanical study of the final push-pull in archery. Journal of Sports Sciences, v. 11, n. 1, p. 63-69, 1993.

LESLIE, M. "The man who stopped time." 2001.

LIN, J. et al. Activation and tremor of the shoulder muscles to the demands of an archery task. Journal of sports sciences, v. 28, n. 4, p. 415-421, 2010. 
LIPSITZ, L. A. Dynamics of stability: the physiologic basis of functional health and frailty. The Journals of Gerontology Series A: Biological Sciences and Medical Sciences, v. 57, n. 3, p. B115-B125, 2002.

LUDEWIG P.; COOK P: Alterations in Shoulder Kinematics and Associated Activity in People with Symptoms of Shoulder Impingement. Physical Therapy 2000, 80:276292.

MARTIN, P.; HEISE, G. Archery Bow Grip Force Distribution: Relationships With Performance and Fatigue. International Journal of Sport Biomechanics, v. 8, p. 305319, 1992.

MARTIN, P. E.; SILER, W. L.; HOFFMAN, D. Electromyographic analysis of bow string release in highly skilled archers. Journal of sports sciences, v. 8, n. 3, p. 215$21, .1990$.

MILLER, S.; BARTLETT, R.. The relationship between basketball shooting kinematics, distance and playing position. Journal of sports sciences, v. 14, n. 3, p. 243-253, 1996.

MOCHIZUKI, L.; AMADIO, A. C. As funções do controle postural durante a postura ereta. Fisioterapia e Pesquisa, v. 10, n. 1, p. 7-15, 2003.

MORRISS, C.; BARTLETT, R.; FOWLER, N. Biomechanical analysis of the men's javelin throw at the 1995 World Championships in Athletics. World, v. 12, n. 23, p. 3141, 1997.

MOSELEY, G.L.; HODGES, P.W., 2006. Reduced variability of postural strategy prevents normalization of motor changes induced by back pain: a risk factor for chronic trouble? Behavior Neuroscience. 120, 474-476

NEWELL, K. M.; JAMES, E. G. The amount and structure of human movement variability. In: HONG, Youlian; BARTLETT, Roger (Ed.). Routledge handbook of biomechanics and human movement science. Routledge, 2008.

NELSON-WONG, E. et al. Application of autocorrelation and cross-correlation analyses in human movement and rehabilitation research. Journal of orthopaedic \& sports physical therapy, v. 39, n. 4, p. 287-295, 2009.

NISHIZONO, H. et al. Analysis of Archery Shooting Techniques by Means of Electromyography. ISBS-Conference Proceedings Archive. Anais.1987

OSTRY, D. J.; FELDMAN, A. G. A critical evaluation of the force control hypothesis in motor control. Experimental brain research, v. 153, n. 3, p. 275-288, 2003.

ROBERTSON, G. et al. (Eds.). . Research Methods in Biomechanics. 2nd. ed. Champaign, Ill, USA.: Human Kinectics, 2013.

RODRIGUES, P. et al. Evaluating the Coupling Between Foot Pronation and Tibial Internal Rotation Continuously Using Vector Coding. Journal of Applied Biomechanics, v. 31, n. 2, p. 88-94, 2015

SENIAM. Surface ElectromyoGraphy for the Non-Invasive Assessment of Muscles. 2014. 
SHINOHARA, H.; URABE, Y. Analysis of muscular activity in archery: a comparison of skill level. The Journal of sports medicine and physical fitness, 2017.

SIGNORILE, J. E.; ZINK, A. J.; SZWED, S. P. A comparative electromyographical investigation of muscle utilization patterns using various hand positions during the lat pull-down. The Journal of Strength \& Conditioning Research, v. 16, n. 4, p. 539$546,2002$.

SIMSEK, D. et al. Kinetic and kinematic analysis of recurve archery shooting technique. In: ISBS-Conference Proceedings Archive. 2014.

Muscular coordination of movements associated with arrow

release in archery. South African Journal for Research in Sport, Physical Education and Recreation, v. 40, n. 1, p. 141-155, 2018.

SLEIMEN-MALKOUN, R.; TEMPRADO, JJ.; HONG, S. L. Aging induced loss of complexity and dedifferentiation: consequences for coordination dynamics within and between brain, muscular and behavioral levels. Frontiers in aging neuroscience, v. 6 , p. 140, 2014.

SLIJPER, H.; LATASH, M. The effects of instability and additional hand support on anticipatory postural adjustments in leg, trunk, and arm muscles during standing. Experimental brain research, v. 135, n. 1, p. 81-93, 2000.

SOYLU, A. R.; ERTAN, H.; KORKUSUZ, F. Archery performance level and repeatability of event-related EMG. Human movement science, v. 25, n. 6, p. 767-74. 2006.

SPARROW, W. A. et al. Using relative motion plots to measure changes in intra-limb and inter-limb coordination. Journal of motor behavior, v. 19, n. 1, p. 115-129, 1987.

SQUADRONE, R.; RODANO, R.; GALLOZZI, C. Fatigue effects on shooting archery performance. ISBS-Conference Proceedings Archive, v. 1, n. 1, p. 274-277, 1994.

STEFANYSHYN, D. J.; WOROBETS, J. T. Sports equipment-energy and performance. Handbook of Biomechanics and Human Movement Science, Editors Y. Hong and R. Bartlett, Routledge International Handbooks, p. 257-268, 2008.

STERGIOU, Nicholas (Ed.). Innovative analyses of human movement. Human Kinetics Publishers, 2004.

et al. Optimal movement variability: a new theoretical perspective for neurologic physical therapy. Journal of Neurologic Physical Therapy, v. 30, n. 3, p. 120-129, 2006.

; DECKER, L. M. Human movement variability, nonlinear dynamics, and pathology: is there a connection? Human movement science, v. 30, n. 5, p. 86988, out. 2011.

STUART, J.; ATHA, J. Postural consistency in skilled archers. Journal of Sports Sciences, v. 8, n. 3, p. 223-234, 1990.

SUWARGANDA, E. et al. Influence of muscle activity on shooting performance in archery: Preliminary findings. ISBS-Conference Proceedings Archive. Anais...2012 
TAHA, Z. et al. Correlation between Archer's Hands Movement While Shooting and its Score. Procedia engineering, v. 147, p. 145-150, 2016.

TAKAI, H.; KUBO, Y.; ARAKI, M. Characteristics of shooting time of the world's top level male archery athletes. NSSU Journal of Sport Sciences, v. 1, p. 8-12, 2012.

TINAZCI, C. Shooting dynamics in archery: A multidimensional analysis from drawing to releasing in male archers. Procedia Engineering, v. 13, p. 290-296, 2011

TEPAVAC, D; FIELD-FOTE, EC. Vector coding: a technique for quantification of intersegmental coupling in multicyclic behaviors. Journal of Applied Biomechanics, v. 17 , n. 3, p. 259-270, 2001

TODOROV, E.; JORDAN, M. I. Optimal feedback control as a theory of motor coordination. Nature neuroscience, v. 5, n. 11, p. 1226, 2002.

TURVEY, M. T. Coordination. American psychologist, v. 45, n. 8, p. 938, 1990.

UDOFA, Andrew et al. Ground reaction forces during competitive track events: a motion based assessment method. ISBS Proceedings Archive, v. 35, n. 1, p. 120, 2017.

VAN EMMERIK, R; VAN WEGEN, EEH. On the functional aspects of variability in postural control. Exercise and sport sciences reviews, v. 30, n. 4, p. 177-183, 2002.

Dynamical Systems Analysis of Coordination. In: ROBERTSON, G. et al. (Eds.). Research Methods in Biomechanics. 2nd. ed. Champaign, Ill, USA.: Human Kinectics, 2013.

VEREIJKEN, B.; WHITING, H. T. A.; BEEK, W. J. A dynamical systems approach to skill acquisition. The Quarterly Journal of Experimental Psychology, v. 45, n. 2, p. 323-344, 1992.

WEYAND, P. Does symmetry matter for speed? Study finds Usain Bolt may have asymmetrical running gait. SMU Research News. 2017 (link: https://blog.smu.edu/research/2017/06/27/does-symmetry-matter-for-speed-study-findsusain-bolt-may-have-asymmetrical-running-gait/) acessado em 18 de dezembro de 2018

WHEAT, J.S.; GLAZIER, P.S. Measuring coordination and variability in coordination. In DAVIDS, K.; BENNETT, S.; NEWELL, K. M. Movement system variability. Human kinetics, 2006.

WILSON, C. et al. Coordination variability and skill development in expert triple jumpers. Sports biomechanics, v. 7, n. 1, p. 2-9, 2008.

WINTER, D. A. Biomechanics and motor control of human movement. John Wiley \& Sons, 2009. 
I - DADOS DE IDENTIFICAÇÃO DO SUJEITO DA PESQUISA OU RESPONSÁVEL LEGAL

\section{DADOS DO INDIVÍDUO}

Nome completo

$$
\begin{gathered}
\text { Sexo } \\
\text { RG }
\end{gathered}
$$

Data de nascimento

Endereço completo

$$
\begin{aligned}
& \text { CEP } \\
& \text { Fone } \\
& \text { e-mail }
\end{aligned}
$$

\section{RESPONSÁVEL LEGAL}

Nome Completo

$$
\begin{array}{r}
\text { Natureza } \\
\text { Sexo } \\
\text { RG }
\end{array}
$$

Data de nascimento

Endereço completo

$$
\begin{gathered}
\text { CEP } \\
\text { Fone } \\
\text { e-mail }
\end{gathered}
$$

\section{II - DADOS SOBRE A PESQUISA CIENTÍFICA}

1. Título do Projeto de Pesquisa: Atividade elétrica dos músculos dos membros superiores e do tronco no Tiro com Arco2. Pesquisador Responsável: Luis Mochizuki

3. Cargo/Função: Docente

4. Avaliação do risco da pesquisa: Risco Baixo. Os riscos relacionados a pesquisa são de exposição dos arqueiros de elite aos pesquisadores em função do posicionamento de 
eletrodos que devem ser feitos com abrasão da pele e uso de lamina de barbear, possuindo algum incomodo relacionado aos procedimentos experimentais, risco baixo a leve de choque do aparelho eletromiográfico ligado a rede elétrica.

5. Duração da Pesquisa: 2 ano.

6. Protocolo: Agência de Fomento e Número. Financiamento próprio.

\section{III - EXPLICAÇÕES DO PESQUISADOR AO INDIVÍDUO OU SEU REPRESENTANTE LEGAL SOBRE A PESQUISA, DE FORMA CLARA E SIMPLES, CONSIGNANDO:}

1. Este estudo tem como objetivo principal descrever a ação muscular de arqueiros durante o tiro. Os músculos foram escolhidos pela ativação durante os ajustes posturais, no lado dominante flexor superficial dos dedos, extensor dos dedos, bíceps braquial porção longa, tríceps braquial, peitoral maior porção clavicular, reto abdominal porção anterior, serrátil anterior, multífido, latíssimo do dorso e trapézio superior; do lado não dominante os músculos serrátil anterior e deltoide porção posterior. O conhecimento da ação destes músculos pode trazer a elaboração de estratégias para o desempenho esportivo.

2. O voluntário terá 12 canais e 25 eletrodos fixados ao seu corpo para a captação de sinais com eletromiografia e 1 eletrodo terra. Todos os tiros serão gravados a fim de sincronizar a imagem com o aparelho de eletromiografia, e terá um acelerômetro acoplado ao seu punhopara captar o início do movimento e preencherá um questionário da análise de satisfação e estratégia;

3. Não espera-se riscos ou desconfortos com esta pesquisa. Caso o voluntário tenha alguma intercorrência devido aos procedimentos do estudo, o mesmo deve comunicar ao pesquisador responsável;

4. A participação é voluntária, e o referido não receberá quaisquer benefícios em dinheiro ou benefícios de qualquer outro modo;

5. Para dirimir as dúvidas ou esclarecimentos relacionadas a pesquisa, basta entrar em contato, com o pesquisador responsável ou com o Conselho de Ética em Pesquisa em Seres Humanos da Escola de Artes, Ciências e Humanidades da Universidade de São Paulo. Telefone: 3091-1046. E-mail: cep-each@usp.br. Atendimento: Segunda a Sexta-feira, das 10 horas às 12 horas e das 14 horas às 16 horas. Localização: Sala T14 - I1. Endereço: Rua Arlindo Bettio, 1000. Ermelino Matarazzo. CEP: 03828-080. São Paulo-SP.

\section{IV - ESCLARECIMENTOS DADOS PELO PESQUISADOR SOBRE} GARANTIAS DO SUJEITO DA PESQUISA:

1. Todos os experimentos serão realizados de forma não invasiva. Em qualquer etapa deste estudo você terá acesso aos profissionais responsáveis para esclarecimento de eventuais dúvidas;

2. Em caso de recusa você não será penalizado(a) de forma alguma. Além disso, você tem liberdade de retirar seu consentimento e deixar de participar do estudo a qualquer momento, sem que isto traga qualquer prejuízo; 
3. Desde logo fica garantido a confidencialidade, sigilo e privacidade das informações, com os dados divulgados apenas em artigos e eventos científicos, garantindo o sigilo de identidade do sujeito da pesquisa;

4. Você receberá dos pesquisadores todo o apoio e assistência e acompanhamento por eventuais danos à saúde, decorrentes da pesquisa.

\section{V - INFORMAÇÕES DE NOMES, ENDEREÇOS E TELEFONES DOS RESPONSÁVEIS PELO ACOMPANHAMENTO DA PESQUISA, PARA CONTATO EM CASO DE INTERCORRÊNCIAS CLÍNICAS E REAÇÕES ADVERSAS.}

Pesquisador Responsável: Dr. Luis Mochizuki

Endereço: Rua Arlindo Bettio, 1000. Sala 319D. Ermelino Matarazzo. CEP: 03828-080. São Paulo-SP.

Instituição: Escola de Artes, Ciências e Humanidades da Universidade de São Paulo

Telefones para contato: (11) 3091-8805

Pesquisadores gerentes: Fernando Carvalheiro Reiser (11)983.484.135, Nadjila Tejo Machado (11) 985.224.450

\section{VI - CONSENTIMENTO PÓS-ESCLARECIDO}

Declaro que, após a leitura deste documento e esclarecer as minhas dúvidas conversar com o pesquisador responsável e estar ciente do protocolo, concordo em participar como voluntário do projeto de pesquisa supracitada. Estou ciente dos objetivos da pesquisa, dos procedimentos aos quais serei submetido, dos possíveis danos ou riscos deles provenientes e da garantia de confidencialidade e esclarecimentos sempre que desejar. Diante do exposto expresso minha concordância de espontânea vontade em participar deste estudo.

São Paulo,

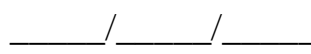

assinatura do sujeito da pesquisa

assinatura do pesquisador

(carimbo ou nome legível)

ou responsável legal

Pesquisador Responsável:

Luis Mochizuki - mochi@usp.br

Discentes: Fernando Carvalheiro Reiser - ray.reiser@gmail.com

Nadjila Tejo Machado - nadjila@folha.com.br 


\section{APÊNDICE II RESUMO APRESENTADO EM CONGRESSO - COBEC}

\section{BCM.121. ELETROMIOGRAFIA E DESEMPENHO DOS MÚSCULOS FLEXOR SUPERFICIAL DOS DEDOS E DO}

EXTENSOR DOS DEDOS EM ARQUEIROS DE ELITE

Reiser FC ${ }^{1}$; Machado $\mathrm{NT}^{1}$; Aoki $\mathrm{MS}^{1}$; Magalhães $\mathrm{FH}^{1}$; Mochizuki L ${ }^{1}$

1 Laboratório de Ciências da Atividade Fisica, Escola de Artes, Ciências e Humanidades, Universidade de São Paulo

Introdução: $\mathrm{O}$ tiro com arco requesita força e resistência de membros superiores e tronco. $\mathrm{O}$ atleta proeficiente atira um conjunto de flechas para o alvo em um curto intervalo com máxima acurácia e precisão ${ }^{1}$. Os músculos do antebraço tem sido estudados extensivamente nesta modalidade. Arqueiros habilidosos tem menor co-contração entre flexores e extensores do antebraço e menor tempo de reação ${ }^{1,2,3}$. Contudo, a relação entre o desempenho no tiro e atividade muscular é pouco conhecida.

Objetivo: Verificar a atividade muscular dos flexores e extensores dos dedos em comparação ao desempenho obtido no tiro com arco.

Métodos: Cinco arqueiros de elite (27,8 \pm 4,7 anos, tempo de experiência: $14,2 \pm$ 4,8 anos, pontuação FITA: 1317 \pm 19,6, máximo 1440) participaram neste estudo. Eletrodos bipolares $(\mathrm{Ag} / \mathrm{AfCl})$ foram posicionados no ventre muscular paralelo as fibras dos músculos; Flexor Superficial dos Dedos e Extensor dos Dedos. Foram analisadas 225 flechas dividas em 75 escores Oito, 75 escores Nove e 75 escores Dez. A atividade muscular foi gravado durante o periodo de $500 \mathrm{~ms}$ antes e $500 \mathrm{~ms}$ depois da queda do clicker. Os dados foram adquiridos por um eletromiográfo 1610C (EMG Systems Brasil(ß)). O sinal de ativação muscular teve média removida, retificado e normalizado por meio de rotinas no MatLab 7.9.0 (R2009b) para o cálculo do valor integral do EMG. A análise estatística foi realizada no software SPSS 23.0, com analíse de variância (ANOVA) de um fator e teste Post hoc Tukey. O nível de significância foi p<0,05.

Resultados: A ativação do músculo Flexor Superficial dos dedos não foi diferente entre os escores ( $\mathrm{p}>0,05$ ). A ativação i

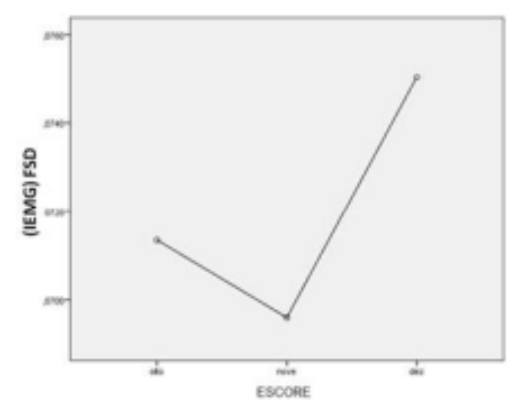

Figura 1 - Intensidade de ativaçào do músculo flexor superficial dos dedos (FSD) nos escores 8, 9 e 10.

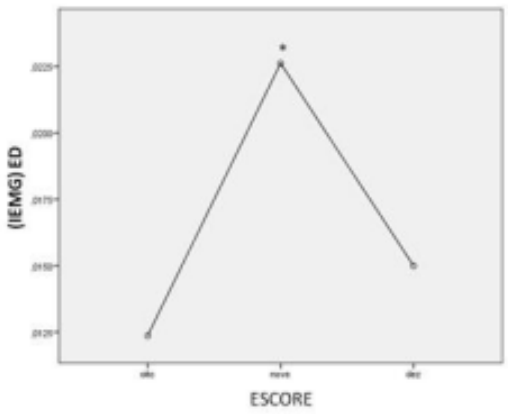

Figura 2 - Intensidade de ativaçào do músculo extensor dos dedos (ED) nos escores 8, 9 e 10.

Discussão: O músculo Extensor dos Dedos apresentou diferença na atividade muscular entre os escores, com o menor recrutamento no escore Oito, e maior no escore Nove. A atividade muscular do escore Dez ficou entre os dois extremos, o que mostra que para obter melhores desempenhos é necessário um tiro com atividade moderada. Dois padròes diferentes de atividade dos músculos extensores dos dedos após a queda do clicker; tem sido mostrados na literatura 1,2,3: relaxamento (silêncio) da musculatura e contraçào ativa ${ }^{1,2}$. Este estudo mostra que existe a atividade muscular moderada, entre o relaxamento e máxima ativação, está contemplada ao maior escore obtido. A variabilidade de atividade muscular entre os escores podem estar 
diretamente relacionadas com o desempenho em demais atividades esportivas, outros músculos e variáveis devem ser investigados para demonstrar este fenômeno efetivamente.

Conclusôes: O nivel da atividade muscular é diferenciado através dos diferentes desempenhos obtidos no tiro com arco, contudo isso só foi mostrado no músculo Extensor dos Dedos.

Comitê de ética e pesquisa: 1.452 .196

Referências

1 Ertan H. et al. Hum Mov; 2003: 22;1;37-45. doi:10.1016/S0167-9457(02)00176-8

2 Martin P. et al. J Sport Sci. 1990: 8;3; 215-221 doi:10.1080/02640419008732147

3 Solyu et al. Hum Mov Sci; 2006: 25;6; 764-777 doi: 10.1016/j.humov.2006.05.002

Fonte dos dados: Esses dados são parte do conteúdo da Dissertaçào de Mestrado intitulada: Utilizaçào da eletromiografia na

variabilidade entre blocos de combate em arqueiros de elite. O trabalho é desenvolvido no Programa de Pós-Graduação em Ciências da Atividade Fisica, EACH-USP. 


\section{POSTER PRESENTATION}

Shoulder and Trunk Muscles Activity in Experienced Archers

Elite performance

"Fernando Carvalheiro Reiser, Nadjila Tejo Machado, Marcelo Saldanha Aoki, Fernando Henrique Magalhães, Luis Mochizuki"

"Universidade of São Paulo, Universidade of São Paulo, Universidade of São Paulo, Universidade of São Paulo, Universidade of São Paulo"

"BR, BR, BR, BR, BR"

\section{"Background:}

Archery requires accuracy and precision to score as high as possible. Strength and endurance of the body, specially, shoulder and trunk muscles, are necessary to maintain the alignment of the bow/arrow system to the target in the draw position. Even experienced archers may not use the same muscle activation along several shots; however, it is unclear how muscle activation changes during an olympics round (72 shots). The aim of this study is to describe electrical activation of upper limbs and trunk muscles of experienced archers during an olympics round.

\section{Methods:}

Four elite archers (age $26.5 \pm 4.5$ years old; with $13.5 \pm 4.5$ years of training; and $1315.75 \pm 23.25$ FाTA points) participated into this study. Electrical activity of several muscles ( $\mathrm{m}$. pectoralis major, $\mathrm{m}$. latissimus dorsi, m. upper trapezius, $\mathrm{m}$. serratus anterior, $\mathrm{m}$. rectus abdominis and $\mathrm{m}$. lumbar multifidus) were recorded ( $2 \mathrm{kHz}$, bipolar, surface and disposable electrodes) during each shot. Archers should shot as accurate and precise as possible 72 arrows to a target $70 \mathrm{~m}$ away, as simulation on olympics competition.

Archers shot 18 arrows as warm-up; then, they have shot 72 arrows (12 ends of 6 arrows, divided into two groups, 3 minutes to walk $140 \mathrm{~m}$ to get the arrows and come back, and 20-minute interval between groups). Electromyography (EMG) data of all muscles was filtered, demeaned and rectified. Integral (epoch: 500ms prior and after the fall of the clicker) of EMG signal was calculated for all muscles and compared across 12 ends. Analysis of variance was run to evaluate the results. Significance level $p<0$, 05.

\section{Results:}

Archer A display no difference in muscle activity between all shots. Archer B, showed differences in the muscle activity of Serratus Anterior $(p=0,006)$, and the Lumbar Multifidus $(p<0,001)$ between the ends. Archer $C$ showed differences in the muscle activity of Serratus Anterior $(p=0,01)$ and the Lumbar pág. 1055 


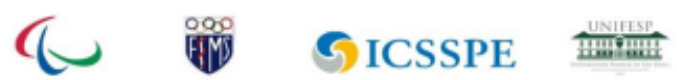

Multifidus $(p=0,04)$ between the ends. $D$ archer showed differences in the muscle activity of Rectus Abdominis $(p<0,001)$, and the Lumbar Multifidus $(p<0,001)$, between the ends. No differences for the Pectoralis Major Clavicular Head, Latissimus Dorsi and Upper Trapezius muscles were been found between all the archers.

\section{Conclusions:}

Elite archers presented different trunk and shoudler girdle muscle activations during 12 ends." 